\title{
Exact WKB analysis and cluster algebras
}

To the memory of Kentaro Nagao

\author{
Kohei Iwaki \\ Research Institute for Mathematical Sciences, Kyoto University, Kyoto, 657-8501, \\ Japan \\ E-mail: iwaki@kurims.kyoto-u.ac.jp
}

\section{Tomoki Nakanishi}

Graduate School of Mathematics, Nagoya University, Chikusa-ku, Nagoya, 464-8602, Japan

E-mail: nakanisi@math.nagoya-u.ac.jp

\begin{abstract}
We develop the mutation theory in the exact WKB analysis using the framework of cluster algebras. Under a continuous deformation of the potential of the Schrödinger equation on a compact Riemann surface, the Stokes graph may change the topology. We call this phenomenon the mutation of Stokes graphs. Along the mutation of Stokes graphs, the Voros symbols, which are monodromy data of the equation, also mutate due to the Stokes phenomenon. We show that the Voros symbols mutate as variables of a cluster algebra with surface realization. As an application, we obtain the identities of Stokes automorphisms associated with periods of cluster algebras. The paper also includes an extensive introduction of the exact WKB analysis and the surface realization of cluster algebras for nonexperts.
\end{abstract}

AMS classification scheme numbers: 13F60,34M60

\section{Contents}

1 Introduction

2 Exact WKB analysis

2.1 Schrödinger equations and associated quadratic differentials . . . . . . 7

2.2 Turning points and singular points . . . . . . . . . . . . . . 8

2.3 Riccati equation . . . . . . . . . . . . . . . . . . . . . . . . . . . . . . . . . . . . .

2.4 WKB solutions . . . . . . . . . . . . . . . . . . . 12

2.5 Borel resummation method and Stokes phenomenon . . . . . . . . . . . . 14

2.6 Trajectories, Stokes curves, and Stokes graphs . . . . . . . . . . . . 17

2.7 Orientation of trajectories . . . . . . . . . . . . . . . . . . . . . . . . . . . . . . . . .

2.8 Borel summability of WKB solutions . . . . . . . . . . . . . . . . . . . . . . . .

2.9 Connection formula for WKB solutions . . . . . . . . . . . . . . . . 26 
3 Voros symbols and Stokes automorphisms 28

3.1 Homology groups and Voros symbols . . . . . . . . . . . . . . 28

3.2 Saddle class associated with saddle trajectory . . . . . . . . . . . 30

3.3 Saddle reduction . . . . . . . . . . . . . . . . . . . . . 30

3.4 Jump formula and Stokes automorphism for regular saddle trajectory . . 32

3.5 Jump formula and Stokes automorphism for degenerate saddle trajectory $\quad \underline{33}$

$3.6 S^{1}$-action on potential and jump formulas . . . . . . . . . . . 34

4 Cluster algebras with coefficients

4.1 Semifields . . . . . . . . . . . . . . . . . . . 37

4.2 Mutation of seeds and cluster algebra with coefficients . . . . . . . . 38

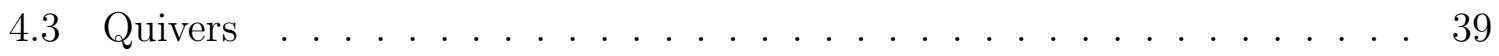

4.4 Tropicalization of $y$-variables and tropical sign . . . . . . . . . . . . . . . . . . . . . . . . . . . . .

$4.5 \varepsilon$-expression of exchange relations . . . . . . . . . . . . . . . . . . . . . . . . . . . . . . .

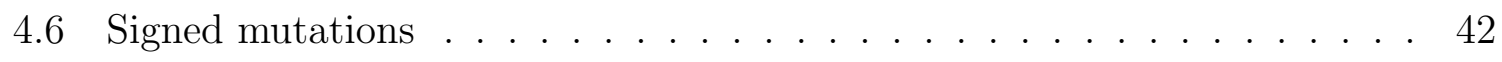

4.7 Periodicity in cluster algebras . . . . . . . . . . . . . . . 43

5 Surface realization of cluster algebras $\quad 45$

5.1 Ideal triangulations of bordered surface with marked points . . . . . . . . 45

5.2 Tagged triangulations . . . . . . . . . . . . . . . . . . . . . . . . . . . . . .

5.3 Realization of exchange graph of labeled seeds . . . . . . . . . . . . 51

5.4 Reformulation by signed triangulations . . . . . . . . . . . . . . . . . . . . . . . . . . . . . .

5.5 Local rescaling and signed pops of extended seeds . . . . . . . . . . . 55

6 Mutation of Stokes graphs

6.1 Stokes triangulations, signed flips, and signed pops . . . . . . . . . 57

6.2 Construction of Stokes triangulation from Stokes graph . . . . . . . . . . 59

6.3 Signed flips and signed pops of Stokes graphs . . . . . . . . . . . . . . . 63

6.4 Simple paths and simple cycles . . . . . . . . . . . . . . . . . . . . . . . . . . .

6.5 Mutation of simple paths and simple cycles . . . . . . . . . . . . . 69

6.6 Periodicity of signed mutations and signed flips . . . . . . . . . . 71

7 Mutation of Voros symbols $\quad 75$

7.1 Mutation formula of Voros symbols for signed flips . . . . . . . . . . . . 75

7.2 Mutation formula of Voros symbols for signed pops . . . . . . . . . . 80

8 Application: Identities of Stokes automorphisms $\quad 83$

8.1 Regular deformation and mutation of potentials . . . . . . . . . . . . . . . .

8.2 Stokes automorphism for general cycle . . . . . . . . . . . . . 85

8.3 Identities of Stokes automorphisms . . . . . . . . . . . 86

A Proof of Theorem 3.4 
B Proof of Theorem $3.7 \quad 92$

B.1 Settings . . . . . . . . . . . . . . . . . . 92

B.2 Derivation of the formulas in Theorem $3.7 \ldots \ldots \ldots$

\section{Introduction}

In this paper we start to develop the mutation theory in the exact WKB analysis using the framework of cluster algebras.

The WKB method was originally initiated by Wentzel, Kramers, and Brillouin in 1926 as the method for obtaining approximate solutions of the Schrödinger equation in the semiclassical limit in quantum mechanics. Voros reformulated the theory based on the Borel resummation method [Vor83], and this new formulation has been further developed by [AKT91, DDP93, etc., and it is called the exact WKB analysis. See the monograph [KT05] for the introduction of the subject. On the other hand, cluster algebras were introduced by Fomin and Zelevinsky around 2000 [FZ02] to study the coordinate rings of certain algebraic varieties and subsequently developed in a series of the papers [FZ03, BFZ05, FZ07]; it was also developed independently by Fock and Goncharov [FG06, FG09a] from the viewpoint of higher Teichmüller theory. It turned out that cluster algebras are "unexpectedly" related with several branches of mathematics beyond the original scope, for example, representation theories of quivers and quantum groups, triangulated categories, hyperbolic geometry, integrable systems, $T$-systems and $Y$-systems, the classical and quantum dilogarithms, Donaldson-Thomas theory, and so on. See the excellent surveys [Kel10, Kel11] for the introduction of the subject.

Let us quickly explain the intrinsic reason why the above seemingly unrelated two subjects are closely related. Let us consider the Schrödinger equation on a compact Riemann surface $\Sigma$

$$
\left(\frac{d^{2}}{d z^{2}}-\eta^{2} Q(z, \eta)\right) \psi(z, \eta)=0,
$$

where $z$ is a local complex coordinate of $\Sigma, \eta=\hbar^{-1}$ is a large parameter, and the potential $Q(z, \eta)$ is a function of both $z$ and $\eta$. The principal part $Q_{0}(z)$ of $Q(z, \eta)$ in the power series expansion in $\eta^{-1}$ defines a meromorphic quadratic differential $\phi$ on $\Sigma$. The trajectories of the quadratic differential $\phi$ determine a graph $G$ on $\Sigma$ called the Stokes graph of the equation (1.1), which plays the central role in the exact WKB analysis. On the other hand, the Stokes graph $G$ can be translated into a triangulation $T$ of the surface $\Sigma$ (with holes and punctures) [KT05, GMN13, BS13]. Due to the works by Gekhtman, Shapiro, and Vainshtein GSV05], Chekhov, Fock, and Goncharov (FG06], FG07] for a review), and Fomin, Shapiro, and Thurston [FST08, FT12], the triangulation $T$ is further identified with a seed $(B, x, y)$ of a certain cluster algebra, which is the main object in cluster algebra theory.

Our main purpose is to develop the mutation theory in the exact WKB analysis. Under a continuous deformation of the potential $Q(z, \eta)$, the Stokes graph may change its 


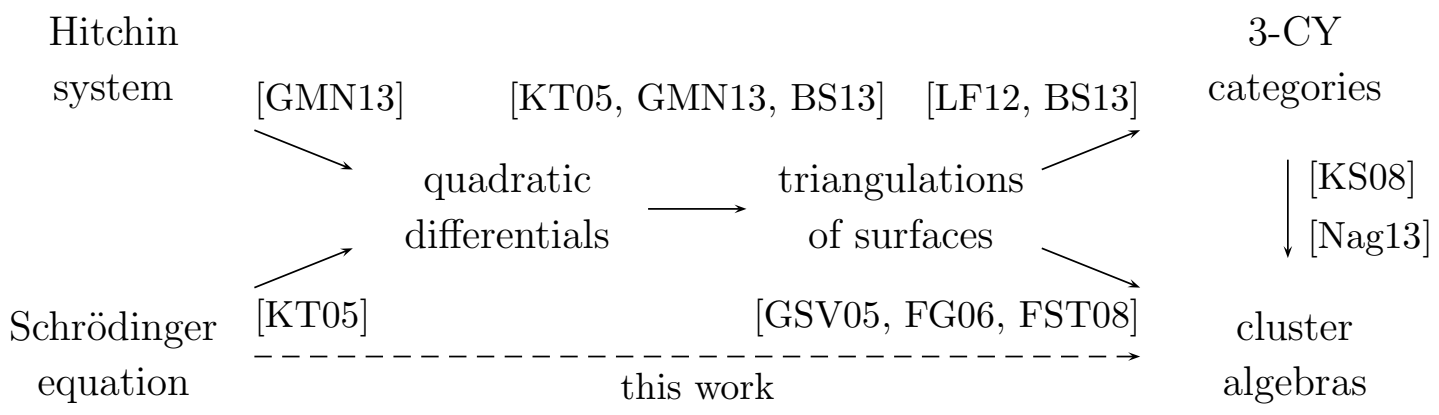

Figure 1. Outlines of previous and this works

topology. We call this phenomenon the mutation of Stokes graphs, since they correspond to the mutation of triangulations through the above correspondence. Along the mutation of Stokes graphs, the monodromy data of the equation (1.1) called the Voros symbols, also mutate [DDP93, DP99]. It turns out that this precisely coincides with the mutation of seeds of the corresponding cluster algebra. In short, this is the main result of the paper.

Before going into further detail of the results, let us mention previous works closely related to this work. Our results have remarkable overlaps and resemblance with the wall-crossing formula of the Donaldson-Thomas invariants and quantum dilogarithm identities, since they are also related with (quantum) cluster algebras [FG09b, KS08, KS10, Nag13, Kel11, Nag11, KN11]. To understand the BPS spectrum of the $d=4, \mathcal{N}=2$ field theories, Gaiotto, Neitzke, and Moore GMN13 studied the WKB approximation for the flat connections of the Hitchin system on a Riemann surface, and its mutation theory. The Stokes graph naturally appeared also in their study, and, in particular, they clarified that there are two types of "elementary mutations" of Stokes graphs, namely, flips and pops. They also identify certain quantities for the Hitchin system as the $y$-variables (the "Fock-Goncharov coordinate" therein) in cluster algebras. See Xie12, Cir13, for example, for a recent development. The mutation aspect of Stokes graphs was further developed by Bridgeland and Smith [BS13]; their aim was the construction of the stability condition in the 3-Calabi-Yau categories associated with surface triangulations based on the work of Labardini-Fragoso [LF12]. The connection between such 3-Calabi-Yau categories and cluster algebras were studied by [KS08, Nag13]. In this paper we will rely on the result of [BS13] for the mutation property of Stokes graphs. We are also motivated by Kontsevich and Soibelman's observation that "There is a striking similarity between our [their] wall-crossing formula and identities for the Stokes automorphisms in the theory of WKB asymptotics..." [KS10, Section 7.5]. (See (1.2) and (1.3) below.) Our result provides an understanding of this similarity at the level of cluster algebras. We summarize the relation of previous and this works schematically in Figure 1 .

For those who are familiar with the subject, let us give a little more extended summary of our results and also present some keywords without definitions. The readers 


\begin{tabular}{ll}
\hline exact WKB analysis & cluster algebra \\
\hline signed flip of Stokes graph & signed mutation \\
signed pop of Stokes graph & signed pop (local rescaling) \\
simple path & monomial $x$-variable \\
simple cycle & monomial $y$-variable \\
Voros symbol for simple path & $x$-variable in extended seed \\
Voros symbol for simple cycle & $\hat{y}$-variable in extended seed \\
\hline
\end{tabular}

Table 1. Dictionary between exact WKB analysis and cluster algebras.

can safely skip this summary, and come back later when the materials are discussed. In any case Figure 1 and Table 1 should be a useful guide to read through the paper.

(a). Signed flips and signed pops. The mutation property of Stokes graphs itself is purely geometrical. Here, we consider two kinds of elementary mutations, flips and pops. To be precise, there are two ways to do flips and pops, namely, to do them clockwise and anticlockwise. We call them signed flips and signed pops. Accordingly, we need to extend the usual notions of tagged triangulations (or equivalently, signed triangulations) and seeds to what we call Stokes triangulations and extended seeds. Then, we define the signed flips (signed mutations for seeds) and signed pops for Stokes triangulations and extended seeds.

(b). Local result: Mutations of simple paths, simple cycles, and Voros symbols. Let $\hat{\Sigma}$ be the covering of the surface $\Sigma$ to make the square root of the quadratic differential $\phi$ single valued. We introduce the simple paths and the simple cycles, which are certain elements of the relative homology and the homology of $\hat{\Sigma}$. Under the mutation of Stokes graphs, they transform (= mutate) as monomial $x$-variables and monomial y-variables, which are ingredients in our extended seeds (Proposition 6.28). We consider the Voros symbols associated with the simple paths and the simple cycles. As formal series in the parameter $\eta^{-1}$, they mutate according to the mutations of the simple paths and the simple cycles. In addition, by the Borel resummation the Voros symbols suffer nontrivial jumps along flips and pops of Stokes graphs due to the Stokes phenomenon. The jump formula was known for flips (Theorem 3.4) earlier by [DDP93, DP99], and we call it the Delabaere-Dillinger-Pham (DDP) formula. An analogous formula for pops (Theorem 3.7) are recently given by [AIT] in conjunction with this work. Combining these geometric and analytic results, we conclude that the Voros symbols for the simple paths mutate as $x$-variables in our extended seeds, while the Voros symbols for the simple cycles mutate as $\hat{y}$-variables therein (Theorem 7.11). This is our first main result. The correspondence between the data in the exact WKB analysis and cluster algebras are summarized in Table 1. We note that much of our efforts are spent to work on pops. In particular, if we concentrate on flips, the setting becomes much lighter.

(c). Global result: Identities of Stokes automorphisms. According to [DDP93], the mutation formula of the Voros coefficients in (b) can be rephrased in terms of the Stokes 
automorphisms acting on the field generated by the Voros symbols. It is known that cluster algebras have a rich periodicity property. Thanks to our result (b), a periodicity in cluster algebras implies an identity of Stokes automorphisms (Theorem 8.6). As the simplest example, if we apply it for the celebrated periodicity of flips of triangulations of a pentagon with period 5 (Figure 20), we have the identity in [DDP93]:

$$
\mathfrak{S}_{\gamma_{2}} \mathfrak{S}_{\gamma_{1}}=\mathfrak{S}_{\gamma_{1}} \mathfrak{S}_{\gamma_{1}+\gamma_{2}} \mathfrak{S}_{\gamma_{2}}
$$

where $\mathfrak{S}_{\gamma}$ is the Stokes automorphism for a cycle $\gamma$. Our identities give a vast generalization of the identity (1.2). This is our second main result. We note that a quantum dilogarithm identity is also associated with the same periodicity of the cluster algebra [Kel11, Nag11, KN11]. For example, the quantum dilogarithm identity associated with the same period of a pentagon gives the celebrated pentagon identity by [FK94, and it looks as follows:

$$
\Psi_{q}\left(U_{2}\right) \Psi_{q}\left(U_{1}\right)=\Psi_{q}\left(U_{1}\right) \Psi_{q}\left(q^{-1} U_{2} U_{1}\right) \Psi_{q}\left(U_{2}\right),
$$

where $\Psi_{q}(x)=\prod_{k=0}^{\infty}\left(1+x q^{2 k+1}\right)$ is the quantum dilogarithm, and $U_{2} U_{1}=q^{2} U_{1} U_{2}$. This is also interpreted as the simplest example of the wall-crossing formula of the DonaldsonThomas invariant in [KS08, KS10]. The similarity between the identities (1.2) and (1.3) is the one observed by [KS10]. Our derivation of (1.2) based on a periodicity of a cluster algebra naturally explains the similarity. It is desirable to understand the similarity at a deeper level, and we leave it as a future problem.

Let us explain the organization of the paper. We anticipate that most of the readers are unfamiliar with at least one of two main subjects, the exact WKB analysis or cluster algebras and their surface realization. So we provide an extensive introduction of both subjects through Sections 2-5, while setting up the formulation we will use. In Section 2 we review the theory of the exact WKB analysis, mainly following [KT05]. Furthermore, we extend the method to a general compact Riemann surface. In Section 3 we introduce an important notion in the exact WKB analysis, called the Voros symbols. We discuss the jump property of the Voros symbols caused by the Stokes phenomenon relevant to the appearance of saddle trajectories in the Stokes graph. In Section 4 we introduce the basic notions and properties in cluster algebras which we will use later. In Section 5 the surface realization of cluster algebras by GSV05, FG06, FST08, FT12] is reviewed. Since careful treatment of mutations involving a self-folded triangle is crucial throughout the paper, we explain in detail how there are related to tagged triangulations and signed triangulations. The extended seeds and their signed mutations and pops are also defined.

Then, we start to integrate these two methods from Section 6. In Section 6 we study the mutation of Stokes graphs, which is purely geometric. We introduce Stokes triangulations, and their signed flips and pops. They effectively control the mutation of Stokes graphs. We introduce the simple paths and the simple cycles of a Stokes graph, and give their mutation formulas. In Section 7 we combine the analytic and geometric results in Sections 3 and 6 and show that the Voros symbols for the simple paths and 
the simple cycles mutate exactly as $x$-variables and $\hat{y}$-variables in our extended seeds. In Section 8 by combining all results in the previous sections we derive the identities of Stokes automorphisms associated with periods of seeds in cluster algebras.

Acknowledgements. We are grateful to Tatsuya Koike and Reinhard Schäfke to sharing their result before publication. We thank Takashi Aoki, Yuuki Hirako, Kazuo Hosomichi, Akishi Ikeda, Takahiro Kawai, Alastair King, Hirokazu Maruhashi, Andrew Neitzke, Michael Shapiro, Ivan Smith, Toshinori Takahashi, Yoshitsugu Takei, and Dylan Thurston for useful discussions and communications. The first author is supported by Research Fellowships of Japan Society for the Promotion for Young Scientists. We dedicate the paper to the memory of Kentaro Nagao, who inspired us by his beautiful papers, talks, and private conversations at various occasions.

\section{Exact WKB analysis}

In this section we review the theory of the exact WKB analysis ([Vor83]). Most of our notations are consistent with those of [KT05]. Usually, in the exact WKB analysis the Schrödinger equation is studied on the Riemann sphere $\mathbb{P}^{1}$. Here, we extend the method to general compact Riemann surfaces.

\subsection{Schrödinger equations and associated quadratic differentials}

Let $\Sigma$ be a compact Riemann surface, by which we mean a compact, connected, and oriented Riemann surface throughout the paper.

Consider a differential equation $\mathcal{L}: L \psi=0$ for a function $\psi$ on $\Sigma$. Here $L=L(z, d / d z, \eta)$ is a second order linear differential operator with meromorphic coefficients and containing a large parameter $\eta$. We usually regard $\eta$ as a real (positive) large parameter, but sometimes regard it as a complex large parameter. Assume that, in a local complex coordinate $z$ of $\Sigma, \mathcal{L}$ is represented as follows:

$$
\mathcal{L}: L \varphi=\left(\frac{d^{2}}{d z^{2}}-\eta^{2} Q(z, \eta)\right) \psi(z, \eta)=0,
$$

where

$$
Q(z, \eta)=Q_{0}(z)+\eta^{-1} Q_{1}(z)+\eta^{-2} Q_{2}(z)+\cdots
$$

is a polynomial in $\eta^{-1}$ (i.e., $Q_{n}(z)=0$ for $n \gg 1$ ) whose coefficients $\left\{Q_{n}(z)\right\}_{n \geq 0}$ are meromorphic functions on $\Sigma$. We remark that any ordinary differential equation of the form

$$
\left(\frac{d^{2}}{d z^{2}}+\eta p(z, \eta) \frac{d}{d z}+\eta^{2} q(z, \eta)\right) \varphi(z, \eta)=0
$$

can be reduced to the form (2.1) by a certain gauge transformation. The equation (2.1) is nothing but a one-dimensional stationary Schrödinger equation, where $\eta^{-1}$ corresponds to the Planck constant $\hbar$, with the potential function $Q(z, \eta)$ whose principal term is 
given by $Q_{0}(z)$. We will impose some assumptions on the potential $Q(z, \eta)$ in subsequent subsections.

We call (2.1) the Schrödinger form (of $\mathcal{L}$ ) in the local coordinate $z$, since the potential function $Q(z, \eta)$ depends on the choice of the local coordinate. If we take a coordinate transformation $z=z(\tilde{z})$ and a gauge transformation, the Schrödinger form in the local coordinate $\tilde{z}$ becomes

$$
\begin{gathered}
\left(\frac{d^{2}}{d \tilde{z}^{2}}-\eta^{2} \tilde{Q}(\tilde{z}, \eta)\right) \tilde{\psi}(\tilde{z}, \eta)=0, \quad \tilde{\psi}(\tilde{z}, \eta)=\psi(z(\tilde{z}), \eta)\left(\frac{d z(\tilde{z})}{d \tilde{z}}\right)^{-1 / 2} \\
\tilde{Q}(\tilde{z}, \eta)=Q(z(\tilde{z}), \eta)\left(\frac{d z(\tilde{z})}{d \tilde{z}}\right)^{2}-\frac{1}{2} \eta^{-2}\{z(\tilde{z}) ; \tilde{z}\}
\end{gathered}
$$

where $\{z(\tilde{z}) ; \tilde{z}\}$ is the Schwarzian derivative

$$
\{z(\tilde{z}) ; \tilde{z}\}=\left(\frac{d^{3} z(\tilde{z})}{d \tilde{z}^{3}} / \frac{d z(\tilde{z})}{d \tilde{z}}\right)-\frac{3}{2}\left(\frac{d^{2} z(\tilde{z})}{d \tilde{z}^{2}} / \frac{d z(\tilde{z})}{d \tilde{z}}\right)^{2} .
$$

In particular, the transformation law

$$
\tilde{Q}_{0}(\tilde{z})=Q_{0}(z(\tilde{z}))\left(\frac{d z}{d \tilde{z}}\right)^{2}
$$

of the principal terms of the potential functions of the Schrödinger form coincides with that of a meromorphic quadratic differential, that is, a meromorphic section of the line bundle $\omega_{\Sigma}^{\otimes 2}$. Here $\omega_{\Sigma}$ is the holomorphic cotangent bundle on $\Sigma$.

Definition 2.1. The quadratic differential associated with $\mathcal{L}$ is the meromorphic quadratic differential on $\Sigma$ which is locally given by

$$
\phi=Q_{0}(z) d z^{\otimes 2}
$$

in a local coordinate $z$. Here $Q_{0}(z)$ is the principal term of the potential function $Q(z, \eta)$ of the Schrödinger form of $\mathcal{L}$ in the local coordinate $z$.

Geometry of zeros, poles, and trajectories of $\phi$ are important in the exact WKB analysis. They relate to properties of solutions of $\mathcal{L}$ deeply.

\subsection{Turning points and singular points}

The poles of the associated quadratic differential $\phi$ are singular points of the differential equation (2.1). In the exact WKB analysis the zeros of $\phi$ are also important.

Definition 2.2. A zero (resp., simple zero) of $\phi$ is called a turning point (resp., simple turning point) of $\mathcal{L}$.

Let $P_{0}$ and $P_{\infty}$ be the set of the zeros and the poles of $\phi$, respectively, and set $P=P_{0} \cup P_{\infty}$. In this paper we always impose the following assumption.

Assumption 2.3. Let $\phi$ be the quadratic differential associated with $\mathcal{L}$. We assume 
- $\phi$ has at least one zero, and at least one pole,

- all zeros of $\phi$ are simple,

- the order of any pole of $\phi$ is more than or equal to 2.

Remark 2.4. The behavior of the WKB solutions around a simple pole was studied by Koi00, and it requires special attention in our problem. We will treat the simple pole case in a separate publication.

The quadratic differentials satisfying the above assumption are called complete Gaiotto-Moore-Neitzke (GMN) differentials in [BS13, Section 2.2]. This assumption makes treatment of trajectories easier. The assumption that all turning points are simple is also reasonable in the exact WKB analysis. For example, Theorem 2.25 below can not be applied for higher order turning points.

In addition to Assumption 2.3, we also impose the following assumption for $Q_{n}(z)$ with $n \geq 1$.

Assumption 2.5. (i). If a point $p \in \Sigma$ is a pole of $Q_{n}(z)$ for some $n \geq 1$, then $p \in P_{\infty}$. (ii). If $\phi$ has a pole $p$ of order $m \geq 3$, then the following condition holds.

$$
\text { (order of } \left.Q_{n}(z) \text { at } p\right)<1+\frac{m}{2} \text { for all } n \geq 1 \text {. }
$$

(iii). If $\phi$ has a pole $p$ of order $m=2$, then the following conditions hold.

- $Q_{n}(z)$ has an at most simple pole at $p$ for all $n \geq 1$ except for $n=2$.

- $Q_{2}(z)$ has a double pole at $p$ and satisfies

$$
Q_{2}(z)=-\frac{1}{4 z^{2}}(1+O(z)) \quad \text { as } z \rightarrow 0,
$$

where $z$ is a local coordinate of $\Sigma$ near $p$ satisfying $z(p)=0$.

Note that the conditions (2.8) and (2.9) are independent of the choice of the local coordinate due to the transformation law (2.5) of Schrödinger forms. These assumptions will be necessary to define an integral of a certain 1-form from a point $p \in P_{\infty}$ (see Proposition 2.8). Moreover, Assumption 2.5 is also used in the proof of the Borel summability of the WKB solutions (see Theorem 2.17). Let us give examples satisfying Assumption 2.5.

Example 2.6. (a). Let $\Sigma=\mathbb{P}^{1}$, and consider the potential $Q(z, \eta)=Q_{0}(z)$ which is independent of $\eta$ and a polynomial in $z$ of degree $m \geq 1$. Then, the quadratic differential $\phi$ has only one pole of order $m+4$ at $\infty$. This is the case that Vor83] and DDP93] considered.

(b). Let $\Sigma=\mathbb{P}^{1}$, and consider the following differential equation:

$$
\begin{gathered}
\left(\frac{d^{2}}{d z^{2}}-\eta^{2} Q(z, \eta)\right) \psi=0, \quad Q(z, \eta)=Q_{0}(z)+\eta^{-2} Q_{2}(z) \\
Q_{0}(z)=\frac{(\alpha-\beta)^{2} z^{2}+2(2 \alpha \beta-\alpha \gamma-\beta \gamma) z+\gamma^{2}}{4 z^{2}(z-1)^{2}}, \quad Q_{2}(z)=-\frac{z^{2}-z+1}{4 z^{2}(z-1)^{2}} .
\end{gathered}
$$


Here $\alpha, \beta$ and $\gamma$ are complex parameters. This equation is equivalent to Gauss' hypergeometric equation and studied in AT13. Under a generic condition for the parameters $\alpha, \beta$ and $\gamma$, the quadratic differential $\phi$ has two simple zeros and three poles of order 2 at $0,1, \infty$. We can easily check that (2.9) is satisfied at each pole.

\subsection{Riccati equation}

Following [KT05, Section 2], to construct the WKB solutions of (2.1), we consider the following auxiliary equation, which is called the Riccati equation associated with (2.1):

$$
\frac{d S}{d z}+S^{2}=\eta^{2} Q(z, \eta)
$$

A solution of (2.1) and that of (2.10) are related as

$$
\psi(z, \eta)=\exp \left(\int^{z} S(z, \eta) d z\right) .
$$

We can construct a formal (series) solution of (2.10) in the following form:

$$
S(z, \eta)=\sum_{n=-1}^{\infty} \eta^{-n} S_{n}(z)=\eta S_{-1}(z)+S_{0}(z)+\eta^{-1} S_{1}(z)+\cdots .
$$

Here "formal series" means formal Laurent series in $\eta^{-1}$. The family of functions $\left\{S_{n}(z)\right\}_{n \geq-1}$ must satisfy the following recursion relation

$$
\left\{\begin{array}{l}
S_{-1}^{2}=Q_{0}(z), \\
2 S_{-1} S_{n+1}+\sum_{\substack{n_{1}+n_{2} \leq n \\
0 \leq n_{j} \leq n}} S_{n_{1}} S_{n_{2}}+\frac{d S_{n}}{d z}=Q_{n+2}(z) \quad(n \geq-1) .
\end{array}\right.
$$

We obtain two families of functions $\left\{S_{n}^{(+)}(z)\right\}_{n \geq-1}$ and $\left\{S_{n}^{(-)}(z)\right\}_{n \geq-1}$ which satisfy the recursion relation (2.12), depending on the choice of the root $S_{-1}= \pm \sqrt{Q_{0}(z)}$ for the initial condition in (2.12). Thus we have two formal solutions

$$
S^{( \pm)}(z, \eta)=\sum_{n=-1}^{\infty} \eta^{-n} S_{n}^{( \pm)}(z)= \pm \eta \sqrt{Q_{0}(z)}+\cdots
$$

of the Riccati equation (2.10). The functions $\left\{S_{n}^{( \pm)}(z)\right\}_{n \geq-1}$ are singular on $P$, and multi-valued and holomorphic on $\Sigma \backslash P$.

Following [KT05, Remark 2.2], we define the odd part and the even part of $S(z, \eta)$ by

$$
S_{\text {odd }}(z, \eta)=\frac{1}{2}\left(S^{(+)}(z, \eta)-S^{(-)}(z, \eta)\right), \quad S_{\text {even }}(z, \eta)=\frac{1}{2}\left(S^{(+)}(z, \eta)+S^{(-)}(z, \eta)\right) .
$$

These quantities have the following properties. 
Proposition 2.7. (a). The equality

$$
S^{( \pm)}(z, \eta)= \pm S_{\text {odd }}(z, \eta)+S_{\text {even }}(z, \eta)
$$

holds, and the even part is given by the logarithmic derivative of the odd part:

$$
S_{\text {even }}(z, \eta)=-\frac{1}{2 S_{\text {odd }}(z, \eta)} \frac{d S_{\text {odd }}(z, \eta)}{d z}
$$

(b). The (formal series valued) 1-form $S_{\text {odd }}(z, \eta) d z$ is invariant under coordinate transformations. That is, the odd part $\tilde{S}_{\text {odd }}(\tilde{z}, \eta)$ of a formal solution of the Riccati equation associated with (2.4) is given by

$$
\tilde{S}_{\text {odd }}(\tilde{z}, \eta)=S_{\text {odd }}(z(\tilde{z}), \eta) \frac{d z(\tilde{z})}{d \tilde{z}}
$$

if we choose the square root in (2.13) so that the following equality holds (cf. (2.6)):

$$
\sqrt{\tilde{Q}_{0}(\tilde{z})}=\sqrt{Q_{0}(z(\tilde{z}))} \frac{d z}{d \tilde{z}}
$$

Proof. The claims (a) and (b) are proved by the same argument in [KT05, Remark 2.2] and [KT05, Corollary 2.17], respectively.

Proposition 2.7 implies that the 1 -form $S_{\text {odd }}(z, \eta) d z$ is globally defined (but multivalued) on $\Sigma \backslash P$. This is not integrable at a point in $P_{\infty}$ because the principal term $\eta \sqrt{Q_{0}(z)} d z$ is singular. However, under Assumption 2.5, we can show the following fact.

Proposition 2.8. For any point $p \in P_{\infty}$ and any local coordinate $z$ of $\Sigma$ around $p$ such that $z=0$ at $p$, the formal power series valued 1-form defined by

$$
S_{\text {odd }}^{\mathrm{reg}}(z, \eta) d z=\left(S_{\text {odd }}(z, \eta)-\eta \sqrt{Q_{0}(z)}\right) d z,
$$

is integrable at $z=0$. Namely, for any $n \geq 0$, there exists a real number $\ell>-1$ such that

$$
S_{\text {odd }, n}(z)=O\left(z^{\ell}\right) \quad \text { as } z \rightarrow 0 .
$$

Here $S_{\text {odd }, n}(z)$ is the coefficient of $\eta^{-n}$ in the formal series $S_{\text {odd }}(z, \eta)$. Especially, all coefficients of $S_{\mathrm{odd}}^{\mathrm{reg}}(z, \eta)$ are holomorphic at $p$ if it is an even order pole of $\phi$.

Proof. Fix any local coordinate $z$ around $p$ as above. It follows from the recursion relation (2.12) and the definition (2.14) of $S_{\text {odd }}(z, \eta)$ that $S_{0}^{( \pm)}(z)$ and $S_{\text {odd }, 0}(z)$ are given by

$$
S_{0}^{( \pm)}(z)=-\frac{1}{4 Q_{0}(z)} \frac{d Q_{0}(z)}{d z} \pm \frac{Q_{1}(z)}{2 \sqrt{Q_{0}(z)}}, \quad S_{\text {odd }, 0}(z)=\frac{Q_{1}(z)}{2 \sqrt{Q_{0}(z)}}
$$

Then, although $S_{0}^{( \pm)}(z)=O\left(z^{-1}\right)$ as $z \rightarrow 0$, we can show that (2.20) holds for $n=0$ due to Assumption 2.5, Similarly, $S_{1}^{( \pm)}(z)$ is given by

$$
S_{1}^{( \pm)}(z)=\frac{ \pm 1}{2 \sqrt{Q_{0}(z)}}\left(Q_{2}(z)-S_{0}^{( \pm)}(z)^{2}-\frac{d S_{0}^{( \pm)}(z)}{d z}\right) .
$$


Denote by $m$ the pole order of $\phi$ at $p$. If $m \geq 3$, we can verify that $S_{1}^{( \pm)}(z)=O\left(z^{\ell}\right)$ for some $\ell>-1$ since $\sqrt{Q_{0}(z)}=O\left(z^{-m / 2}\right), S_{0}^{( \pm)}(z)=O\left(z^{-1}\right)$ and we have Assumption 2.5 (ii). Hence we have (2.20) for $n=1$. On the other hand, the situation is different when $m=2$. In view of (2.22),$S_{1}^{( \pm)}(z)$ may have a simple pole at $p$ since $\sqrt{Q_{0}(z)}=O\left(z^{-1}\right)$ when $m=2$. However, with the aid of Assumption 2.5 (iii), we can show that $S_{1}^{( \pm)}(z)$ becomes holomorphic because

$$
Q_{2}(z)-S_{0}^{( \pm)}(z)^{2}-\frac{d S_{0}^{( \pm)}(z)}{d z}=O\left(z^{-1}\right)
$$

holds by (2.9) and (2.21). Therefore, we also have (2.20) for $n=1$ in the case $m=2$. The estimate (2.20) for $n \geq 2$ can be shown by the induction from the recursion relation (2.12) and Assumption 2.5. Furthermore, since $\sqrt{Q_{0}(z)}$ is single-valued around $p$ when it is an even order pole of $\phi$, the recursion relation (2.12) also implies that $S_{n}^{( \pm)}(z)$ and $S_{\text {odd }, n}(z)$ are single-valued around $p$ for all $n \geq 0$. Thus, $S_{\text {odd }, n}(z)$ becomes holomorphic at $p$ for all $n \geq 0$ due to (2.20).

We call $S_{\text {odd }}^{\text {reg }}(z, \eta)$ in (2.19) the regular part of $S_{\text {odd }}(z, \eta) . S_{\text {odd }}^{\text {reg }}(z, \eta)$ is a formal power series in $\eta^{-1}$ since the principal term of $S_{\text {odd }}(z, \eta)$ is eliminated. Integrals of $S_{\text {odd }}(z, \eta) d z$ and $S_{\text {odd }}^{\text {reg }}(z, \eta) d z$ on $\Sigma$ are important in the exact WKB analysis.

\subsection{WKB solutions}

Using the relation (2.11) between the solutions of (2.1) and (2.10), and the property (a) in Proposition 2.7, we obtain the following two formal solutions of (2.1):

$$
\psi_{ \pm}(z, \eta)=\frac{1}{\sqrt{S_{\text {odd }}(z, \eta)}} \exp \left( \pm \int^{z} S_{\text {odd }}(z, \eta) d z\right)
$$

Definition 2.9. The formal solutions (2.24) are called the WKB solutions of (2.1).

The integral of $S_{\text {odd }}(z, \eta) d z$ is defined as a term-wise integral for the coefficient of each power of $\eta$. The lower end-point of the integral (2.24) will be discussed later. Since the coefficients of $S_{\text {odd }}(z, \eta) d z$ are multi-valued on $\Sigma \backslash P$, the path of integral in (2.24) should be considered in the Riemann surface $\hat{\Sigma}$ of the multi-valued 1-form $\sqrt{Q_{0}(z)} d z$. To be more explicit, $\hat{\Sigma}$ is given by a section of the cotangent bundle of $\Sigma$ as $\hat{\Sigma}=\left\{(z, \nu) \mid \nu^{2}=\phi\right\} \subset \omega_{\Sigma}$. Then the coefficients of the 1-form $S_{\text {odd }}(z, \eta) d z$ are single-valued on $\hat{\Sigma}$. The projection $\pi: \hat{\Sigma} \rightarrow \Sigma$ is a double cover branching at the simple zeros and the odd order poles of $\phi$.

To visualize $\hat{\Sigma}$, and to determine the branch of the square root in (2.13), we usually take branch cuts on $\Sigma$. A branch cut must connect two branch points of the covering map $\pi$, and each branch point must be an end-point of a branch cut. Such a collection of branch cuts together with a choice of a point $\hat{z} \in \hat{\Sigma}$ give an embedding $\iota: \Sigma \rightarrow \hat{\Sigma}$, which is a piecewise continuous and has a discontinuity on the branch cut, and contains $\hat{z}$ in its image. We call the image of $\Sigma$ by $\iota$ the first sheet, while the complement of 


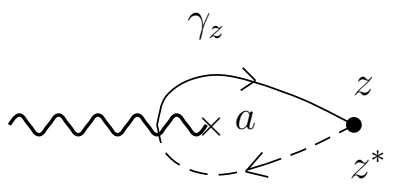

Figure 2. Normalization at a simple turning point.

the first sheet in $\hat{\Sigma}$ the second sheet. We may regard a point on $\Sigma$ as a point on $\hat{\Sigma}$ by such an embedding $\iota$ for a fixed appropriate branch cut, and use the same symbol $z$ for a coordinate of the first sheet, and use $z^{*}=\tau(z)$ for that of the second sheet. Here $\tau: \hat{\Sigma} \rightarrow \hat{\Sigma}$ is the covering involution which exchanges the first and the second sheet, and it commutes with the projection $\pi$. Then, the action of $\tau$ for $S_{\text {odd }}(z, \eta)$ and $S_{\text {odd }}^{\text {reg }}(z, \eta)$ are given by

$$
S_{\text {odd }}\left(z^{*}, \eta\right)=-S_{\text {odd }}(z, \eta), \quad S_{\text {odd }}^{\text {reg }}\left(z^{*}, \eta\right)=-S_{\text {odd }}^{\text {reg }}(z, \eta)
$$

since the involution $\tau$ exchanges the sign in (2.13).

Here we give two well-normalized expressions of the WKB solutions which will be considered in this paper.

- normalized at a turning point $a \in P_{0}$ :

$$
\psi_{ \pm}(z, \eta)=\frac{1}{\sqrt{S_{\text {odd }}(z, \eta)}} \exp \left( \pm \int_{a}^{z} S_{\text {odd }}(z, \eta) d z\right) .
$$

Although the coefficients of $S_{\text {odd }}(z, \eta)$ have a singularity at $a$, the integral (2.26) can be defined with the aid of the anti-invariant property (2.25) of $S_{\text {odd }}(z, \eta)$. Namely, it is defined by the half of the contour integral

$$
\int_{a}^{z} S_{\text {odd }}(z, \eta) d z=\frac{1}{2} \int_{\gamma_{z}} S_{\text {odd }}(z, \eta) d z
$$

along a path $\gamma_{z}$ as in Figure 2, Here the wiggly line designates a branch cut, and the solid part (resp., the dotted part) belongs to the first sheet (resp., the second sheet). In this paper integrals of $S_{\text {odd }}(z, \eta)$ and $S_{\text {odd }}^{\text {reg }}(z, \eta)$ from a simple turning point are always defined in this manner.

- normalized at a pole $p \in P_{\infty}$ :

$$
\psi_{ \pm}(z, \eta)=\frac{1}{\sqrt{S_{\text {odd }}(z, \eta)}} \exp \left\{ \pm\left(\eta \int_{a}^{z} \sqrt{Q_{0}(z)} d z+\int_{p}^{z} S_{\text {odd }}^{\mathrm{reg}}(z, \eta) d z\right)\right\} .
$$

Here $a$ is any turning point independent of $p$. Note that, the integral of $S_{\text {odd }}^{\mathrm{reg}}(z, \eta)$ from a pole $p$ is well-defined by Proposition 2.8. 


\subsection{Borel resummation method and Stokes phenomenon}

Let us expand (2.24) in the following formal series:

$$
\psi_{ \pm}(z, \eta)=\exp \left( \pm \eta \int^{z} \sqrt{Q_{0}(z)} d z\right) \eta^{-1 / 2} \sum_{k=0}^{\infty} \eta^{-k} \psi_{ \pm, k}(z)
$$

It is known that, the series (2.29) is divergent in general, and its "principal term" (see (2.21))

$$
\psi_{ \pm}(z, \eta)=\frac{\eta^{-1 / 2}}{Q_{0}(z)^{1 / 4}} \exp \left( \pm \int^{z}\left\{\eta \sqrt{Q_{0}(z)}+\frac{Q_{1}(z)}{2 \sqrt{Q_{0}(z)}}\right\} d z\right)\left(1+O\left(\eta^{-1}\right)\right)
$$

is known as the Wentzel-Kramers-Brillouin approximation (the WKB approximation) of the solutions of the Schrödinger equation (2.1). (Usually $Q_{1}(z)=0$.) In the framework of the exact WKB analysis we take the Borel resummation of the WKB solutions to obtain analytic results. For the convenience of readers, we give an explanation of the Borel resummation method for formal series in $\eta^{-1}$. See [Cos08] for further explanation.

Definition 2.10. - A formal power series $f(\eta)=\sum_{n=0}^{\infty} \eta^{-n} f_{n}$ in $\eta^{-1}$ is said to be Borel summable if the formal power series

$$
f_{B}(y)=\sum_{n=1}^{\infty} f_{n} \frac{y^{n-1}}{(n-1) !}
$$

converges near $y=0$ and can be analytically continued to a domain $\Omega$ containing the half line $\{y \in \mathbb{C} \mid \operatorname{Re} y \geq 0$, Im $y=0\}$, and satisfies

$$
\left|f_{B}(y)\right| \leq C_{1} e^{C_{2}|y|} \quad(y \in \Omega)
$$

with positive constants $C_{1}, C_{2}>0$. The function $f_{B}(y)$ is called the Borel transform of $f(\eta)$.

- For a Borel summable formal power series $f(\eta)=\sum_{n=0}^{\infty} \eta^{-n} f_{n}$, define the Borel sum of $f(\eta)$ by the following Laplace integral:

$$
\mathcal{S}[f](\eta)=f_{0}+\int_{0}^{\infty} e^{-\eta y} f_{B}(y) d y
$$

Here the path of the integral is taken along the positive real axis. Due to (2.31), the Laplace integral (2.32) converges and gives an analytic function of $\eta$ on $\{\eta \in \mathbb{R} \mid \eta \gg 1\}$.

- Let $f(\eta)=e^{\eta s} \eta^{-\rho} \sum_{n=0}^{\infty} \eta^{-n} f_{n}$ be a formal series with an exponential factor $e^{\eta s}$ for some $\rho \in \mathbb{C}$ and $s \in \mathbb{C}$. $f(\eta)$ is said to be Borel summable if the formal power series $g(\eta)=\sum_{n=0}^{\infty} \eta^{-n} f_{n}$ is Borel summable. The Borel sum of $f(\eta)$ is defined by $\mathcal{S}[f](\eta)=e^{\eta s} \eta^{-\rho} \mathcal{S}[g](\eta)$, where $\mathcal{S}[g]$ is the Borel sum of $g(\eta)$. 
For the simplest example, let us consider the monomial $f(\eta)=\eta^{-n}(n \geq 1)$. Then we have $f_{B}(y)=y^{n-1} /(n-1)$ ! and hence the Borel sum $\mathcal{S}[f](\eta)=\eta^{-n}$ coincides with the original monomial. In general, it is known that, if the formal power series $f(\eta)$ converges and defines a holomorphic function near $\eta=\infty$, then $f(\eta)$ is Borel summable and the Borel sum coincides with the original function $f(\eta)$.

The map $\mathcal{S}$ from a set of Borel summable formal series to a set of analytic functions of $\eta$ is called the Borel resummation operator. The following properties are well-known (e.g., [Cos08, Section 4]).

Proposition 2.11. (a). The operator $\mathcal{S}$ commutes with addition and multiplication. That is, for formal power series $f(\eta)$ and $g(\eta)$ which are Borel summable, we have $\mathcal{S}[f+g]=\mathcal{S}[f]+\mathcal{S}[g], \mathcal{S}[f \cdot g]=\mathcal{S}[f] \cdot \mathcal{S}[g]$.

(b). If a formal power series $f(\eta)$ is Borel summable, then $\mathcal{S}[f](\eta)$ is asymptotically expanded to $f(\eta)$ when $\eta \rightarrow+\infty$.

(c). Let $A(t)=\sum_{k=0}^{\infty} A_{k} t^{k}$ be a convergent series defined near the origin $t=0$. If a formal power series $f(\eta)=\sum_{n=1}^{\infty} \eta^{-n} f_{n}$ without a constant term is Borel summable, then the formal power series $A(f(\eta))=\sum_{k=0}^{\infty} A_{k}(f(\eta))^{k}$ is also Borel summable. Moreover, the Borel sum is given by $\mathcal{S}[A(f(\eta))]=A(\mathcal{S}[f](\eta))$.

Even if a formal power series $f(\eta)$ is divergent, its Borel sum $\mathcal{S}[f](\eta)$ becomes analytic and the original $f(\eta)$ is recovered as an asymptotic expansion of the Borel sum, if $f(\eta)$ is Borel summable. In this sense the Borel resummation method is a natural resummation procedure of divergent series.

However, when the Borel transform $f_{B}(y)$ of $f(\eta)$ has a singular point $y=y_{0}$ on the positive real axis (i.e., $f(\eta)$ is not Borel summable), then the Laplace integral (2.32) can not be defined and we can not find an analytic function of $\eta$ having the above asymptotic property by the "usual" Borel resummation method.

In such a case, to obtain an analytic function which has $f(\eta)$ as its asymptotic expansion when $\eta \rightarrow+\infty$, we regard $\eta$ as a complex large parameter with a certain phase $\arg \eta=\theta \in \mathbb{R}$ and consider the following Borel resummation in the direction $\theta$ :

$$
\mathcal{S}_{\theta}[f](\eta)=f_{0}+\int_{0}^{\infty e^{-i \theta}} e^{-\eta y} f_{B}(y) d y .
$$

Here the path of integral in (2.33) is taken along the half line $\left\{y=r e^{-i \theta} \in \mathbb{C} \mid r \geq 0\right\}$ so that the singular point $y_{0}$ of $f_{B}(y)$ does not lie on the path. If the Laplace integral (2.33) is well-defined in a similar sense of Definition 2.10, then $f(\eta)$ is said to be Borel summable in the direction $\theta$, and $\mathcal{S}_{\theta}$ is called the Borel resummation operator in the direction $\theta$. Then, the analytic continuation of the Borel sum (2.33) becomes an analytic function of $\eta$ in a sector $\{\eta \in \mathbb{C}|| \arg \eta-\theta|<\pi / 2,| \eta \mid \gg 1\}$. Especially, if $f(\eta)$ is Borel summable in the direction $\delta$ for a sufficiently small $\delta>0$, then $\mathcal{S}_{\delta}[f](\eta)$ is analytic on $\{\eta \in \mathbb{R} \mid \eta \gg 1\}$ and having $f(\eta)$ as its asymptotic expansion when $\eta \rightarrow+\infty$. That is, $\mathcal{S}_{\delta}[f](\eta)$ has the desired asymptotic property for large $\eta>0$.

However, there is an ambiguity in analytic functions which are asymptotically expanded to $f(\eta)$ as $\eta \rightarrow+\infty$. Suppose that $f(\eta)$ is Borel summable in the both 
directions $+\delta$ and $-\delta$ for a sufficiently small number $\delta>0$. Then, both of the Borel sums $\mathcal{S}_{ \pm \delta}[f](\eta)$ have the same asymptotic expansion $f(\eta)$ when $\eta \rightarrow+\infty$. But these functions do not coincide in general; if $f_{B}(y)$ has a singular point $y_{0}$ on the positive real axis, the Borel sums $\mathcal{S}_{+\delta}[f](\eta)$ and $\mathcal{S}_{-\delta}[f](\eta)$ may be different since the path of Laplace integrals are not homotopic due to the singular point $y_{0}$.

This is the so-called Stokes phenomenon for the formal series $f(\eta)$. Here the Stokes phenomenon means a phenomenon that, the analytic function which has $f(\eta)$ with its asymptotic expansion when $|\eta| \rightarrow+\infty$ depends on the direction of an approach to $\eta=\infty$, and the analytic functions may be different for different directions in general. Similarly to Proposition $2.11(\mathrm{~b}), \mathcal{S}_{ \pm \delta}[f](\eta)$ is asymptotic to $f(\eta)$ when $|\eta| \rightarrow+\infty$ with $\arg \eta= \pm \delta$. Therefore, the fact that the Borel sums $\mathcal{S}_{+\delta}[f](\eta)$ and $\mathcal{S}_{-\delta}[f](\eta)$ are different implies that the Stokes phenomenon occurs to $f(\eta)$. This is the formulation of the Stokes phenomenon in terms of Borel resummation method. Moreover, the difference of the Borel sums $\mathcal{S}_{ \pm \delta}[f](\eta)$ are exponentially small when $\eta \rightarrow+\infty$ since they have the same asymptotic expansion.

If the formal power series $f(\eta)$ is Borel summable in any direction $\theta$ satisfying $-\delta \leq \theta \leq+\delta$ with a sufficiently small number $\delta>0$, then $f(\eta)$ does not enjoy the Stokes phenomenon; that is, the Borel sums satisfies

$$
\mathcal{S}_{-\delta}[f](\eta)=\mathcal{S}_{+\delta}[f](\eta)=\mathcal{S}[f](\eta)
$$

as analytic functions of $\eta$ on $\{\eta \in \mathbb{R} \mid \eta \gg 1\}$. This is because the Borel transform $f_{B}(y)$ does not have singular points in a domain containing the sector $\left\{y=r e^{-i \theta} \mid r \geq\right.$ $0,-\delta \leq \theta \leq+\delta\}$ and the Laplace integrals (2.33) give the same analytic function. Thus the singular points of the Borel transform $f_{B}(y)$ are closely related to the Stokes phenomenon for the formal series $f(\eta)$.

The following lemma will be used in the subsequent discussions.

Lemma 2.12. Let $f(\eta)=\sum_{n=0}^{\infty} f_{n} \eta^{-n}$ be a formal power series and $\theta$ be a real number. Then, $f(\eta)$ is Borel summable in the direction $\theta$ if and only if the formal power series $f^{(\theta)}(\eta)=\sum_{n=0}^{\infty} f_{n} e^{-i n \theta} \eta^{-n}\left(=f\left(e^{i \theta} \eta\right)\right)$ is Borel summable in the usual sense (i.e., Borel summable in the direction 0$)$.

Lemma 2.12 follows immediately from the equality

$$
f_{B}^{(\theta)}(y)=e^{-i \theta} f_{B}\left(e^{-i \theta} y\right)
$$

that can be shown by a straightforward computation.

When we apply the Borel resummation method to the WKB solutions (2.29), we fix the independent variable $z$ and regard them as formal series in $\eta^{-1}$ with exponential factors $\exp \left( \pm \eta \int^{z} \sqrt{Q_{0}(z)} d z\right)$. Therefore, the condition that "the Borel sum is welldefined" gives a constraint for $z$. The condition can be checked by looking the Stokes graph defined in the next subsection. 


\subsection{Trajectories, Stokes curves, and Stokes graphs}

Let $\phi$ be the quadratic differential associated with $\mathcal{L}$. This subsection is devoted to the description of properties of trajectories of $\phi$. Here a trajectory of $\phi$ is a leaf of the foliation on $\Sigma \backslash P$ defined by the equation

$$
\operatorname{Im} \int^{z} \sqrt{Q_{0}(z)} d z=\text { constant. }
$$

Every point of $\Sigma \backslash P$ lies on a unique trajectory, and any two trajectories are either disjoint or coincide. The foliation structure by the trajectories of $\phi$ has been well studied in Teichmüller theory [Str84]. The relationship between the geometry of trajectories and the asymptotic property of WKB solutions is studied by Fedoryuk [Fed93]. The geometry of trajectories is also important in the exact WKB analysis since we can read off a lot of properties of the WKB solutions, such as the Borel summability (i.e., well-definedness of the Borel sum (2.32) ), from the geometry of the trajectories of $\phi$.

Definition 2.13 ([KT05, Definition 2.6]). A Stokes curve of $\mathcal{L}$ is a trajectory of $\phi$ whose one of the end-points is a turning point of $\mathcal{L}$. Namely, in a local coordinate $z$ of $\Sigma$, the Stokes curves emanating from a turning point $a \in P_{0}$ are defined as

$$
\operatorname{Im} \int_{a}^{z} \sqrt{Q_{0}(z)} d z=0
$$

Note that the Stokes curves are determined from the principal term $Q_{0}(z)$ of the potential function $Q(z, \eta)$ of (2.1). Figure 3 depicts examples of the Stokes curves for several rational functions $Q_{0}(z)$ on $\mathbb{C} \subset \Sigma=\mathbb{P}^{1}$. Here we use the symbol $\times$ for a point in $P_{0}$ (i.e., a turning point) and $\bullet$ for a point in $P_{\infty}$ (i.e., a pole of $\phi$ ) in the figures. The quadratic differentials $\phi$ on $\Sigma$ in these examples have a pole also at $z=\infty$, which is omitted in the figures.

Here we recall some basic properties of the trajectories of $\phi$ from [Str84]. See also [BS13, Section 3] for comprehensible expositions. Firstly, the local foliation structure around simple zeros and poles of order $m \geq 2$ are given below and depicted in Figures 46. For a simple zero $a$, there are exactly three trajectories entering $a$ which are the Stokes curves (Figure 4). For a double pole $p$, there are three cases depending on the residue $r_{p}=\operatorname{Res}_{z=p} \sqrt{Q_{0}(z)} d z$ (Figure 51).

(a). Clockwise or counterclockwise logarithmic spirals wrap onto $p$. This occurs when $r_{p} \notin \mathbb{R} \cup i \mathbb{R}$.

(b). Radial arcs entering $p$. This occurs when $r_{p} \in \mathbb{R}$.

(c). Closed trajectories surround $p$. This occurs when $r_{p} \in i \mathbb{R}$.

For a pole $p$ of order $m \geq 3$, there are exactly $m-2$ asymptotic tangent directions for the trajectories entering $p$ (Figure 6 ).

Secondly, we focus on global properties of the trajectories of $\phi$. It is known that every trajectories fall into exactly one of the following five types ([BS13, Section 3.4]): 


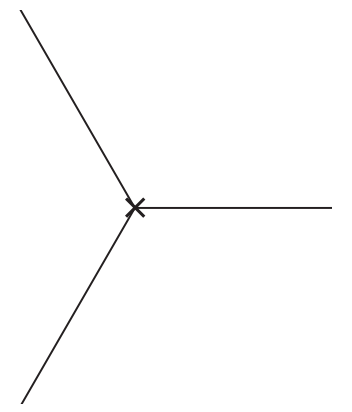

(a) $z$.

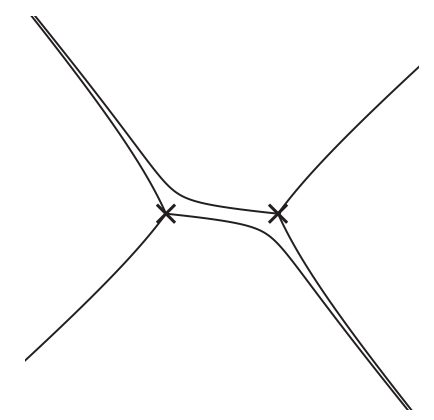

(d) $e^{+\pi i / 10}\left(1-z^{2}\right)$.

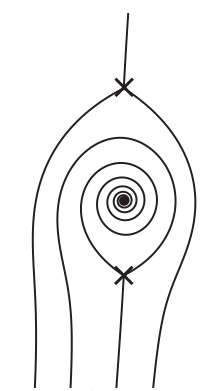

(g) $-e^{+\pi i / 20} \frac{(z+2 i)(z-3 i)}{z^{2}}$.

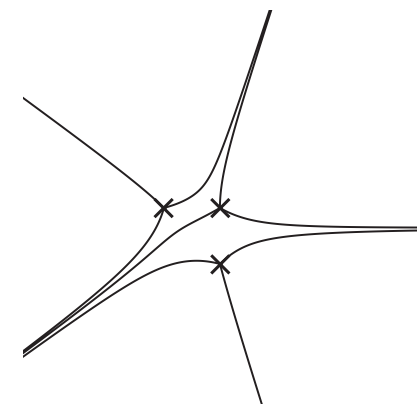

(b) $z(z+1)(z+i)$.

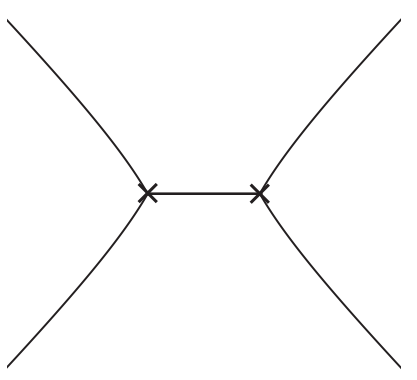

(e) $1-z^{2}$.

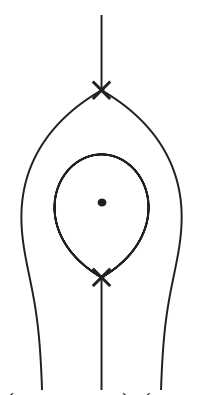

(h) $-\frac{(z+2 i)(z-3 i)}{z^{2}}$.

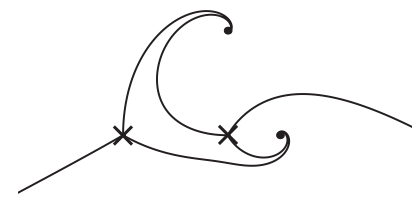

(c) $\frac{z(z+2)}{(z-1)^{2}(z-2 i)^{2}}$.

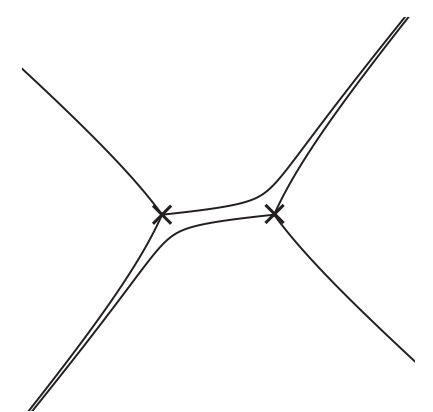

(f) $e^{-\pi i / 10}\left(1-z^{2}\right)$.

Figure 3. Examples of Stokes graphs. The rational functions represent the function $Q_{0}(z)$.

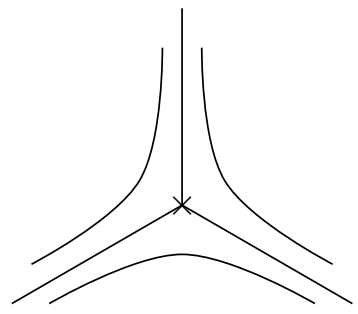

Figure 4. Foliation around a simple zero. 


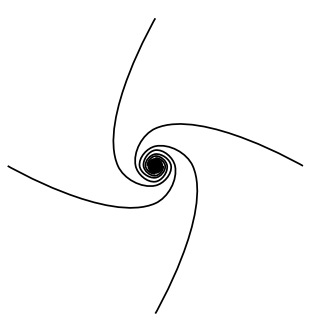

(a)

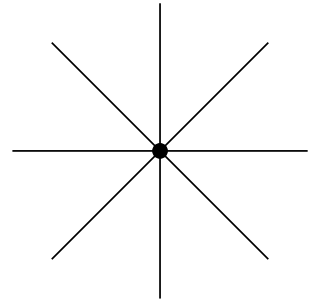

(b)

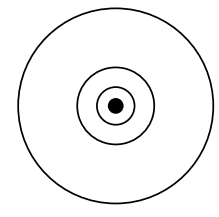

(c)

Figure 5. Patterns of foliation around a double pole.

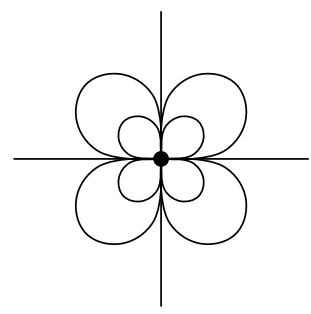

Figure 6. Foliation around a pole of order $m \geq 3$. The case $m=6$ is shown.

(a). A saddle trajectory flows into points in $P_{0}$ at both ends.

(b). A separating trajectory flows into a point in $P_{0}$ at one end, and a point in $P_{\infty}$ at the other end.

(c). A generic trajectory flows into points in $P_{\infty}$ at both ends.

(d). A closed trajectory is a simple closed curve in $\Sigma \backslash P$.

(e). A divergent trajectory has the limit set consisting of more than one point in at least one direction.

A Stokes curve is one of a saddle trajectory, a separating trajectory, and a divergent trajectory.

Saddle trajectories play important roles in this article. Typically, there are two kinds of saddle trajectories:

(a). A regular saddle trajectory connects two different points in $P_{0}$. An example appears in Figure 3 (e).

(b). A degenerate saddle trajectory forms a loop around a double pole $p \in P_{\infty}$. An example appears in Figure $3(\mathrm{~h})$.

In addition to degenerate saddle trajectories, other kinds of loop-type saddle trajectories may appear. For example, a Stokes curve emanating from $a \in P_{0}$ may return to the same point $a$ after encircling several points in $P$. Such an example is discussed in GMN13, Section 10], but we will not consider these cases. In this paper we will concentrate on the following cases:

Assumption 2.14. The number of the saddle trajectories of $\phi$ is at most one. 
Under Assumptions 2.3 and 2.14, a saddle trajectory must be either a regular or a degenerate saddle trajectory (see [BS13, Proposition 10.4]). Moreover, we can show that divergent trajectories never appear in this case.

Lemma 2.15. Under Assumptions 2.3 and 2.14, $\phi$ has no divergent trajectories.

Proof. If $\phi$ does not have any saddle trajectory, then the statement is proved in BS13, Lemma 3.1]. Assume that $\phi$ has a unique saddle trajectory. If a divergent trajectory appears, the interior of the closure of the divergent trajectory gives a domain called a "spiral domain". It is known that the boundary of such a spiral domain must consist of a number of saddle trajectories (see [BS13, Section 3.4]). Since we have assumed that the number of saddle trajectories is exactly one, a domain whose boundary consists of saddle trajectories must be a "degenerate ring domain" (see [BS13, Section 3.4] or below). Then we have a contradiction because any trajectory in a degenerate ring domain must be a closed trajectory, which is not a divergent trajectory.

Therefore, under Assumptions 2.3 and 2.14, a Stokes curve must be a saddle trajectory or a separating trajectory. In other words, a Stokes curve emanating from a turning point must flows into a point in $P$, and these objects define a graph on $\Sigma$.

Definition 2.16 ([KT05, Definition 2.10]). - The Stokes graph of $\mathcal{L}$ is a graph in $\Sigma$ whose vertices are the points in $P$, and whose edges are the Stokes curves of $\mathcal{L}$. The Stokes graph is denoted by $G$.

- The interior of each face of the Stokes graph $G$ is called a Stokes region of $G$.

We sometimes write $G=G(\phi)$ for the Stokes graph and call it the Stokes graph of $\phi$ when we want to emphasize the dependence on $\phi$. If the Stokes graph $G$ does not have any saddle trajectory, $G$ is said to be saddle-free, and then $\phi$ is also said to be saddle-free, following [BS13, Section 3.5]. Under Assumptions 2.3 and 2.14, the Stokes regions of $G$ are classified as follows ([BS13, Section 3.4]):

(a). A horizontal strip is equivalent to a region

$$
\{w \in \mathbb{C} \mid a<\operatorname{Im}(w)<b\} \quad(a, b \in \mathbb{R})
$$

equipped with the differential $d w^{\otimes 2}$. It is swept out by generic trajectories which connect two (not necessarily distinct) poles of arbitrary order $m \geq 2$.

(b). A half plane is equivalent to the upper half plane

$$
\{w \in \mathbb{C} \mid 0<\operatorname{Im}(w)\}
$$

equipped with the differential $d w^{\otimes 2}$. It is swept out by generic trajectories which connect a fixed pole of order $m \geq 3$ to itself.

(c). A degenerate ring domain is equivalent to a region

$$
\{w \in \mathbb{C}|0<| w \mid<a\} \quad(a \in \mathbb{R})
$$


equipped with the differential $r d w^{\otimes 2} / w^{2}$ for some $r \in \mathbb{R}_{<0}$. It is swept out by closed trajectories, and its boundary consists of a degenerate saddle trajectory and the double pole lying inside of the degenerate saddle trajectory.

For example, all three Stokes regions in Figure 3 (a) are half planes. On the other hand, all three Stokes regions in Figure 3 (c) are horizontal strips. In Figure 3 (b) there are five half planes near $z=\infty$ and two horizontal strips. An example of a degenerate ring domain can be found in Figure 3 (h).

In Section 2.8 we will explain the relationship between the geometry of the trajectories of $\phi$ and the Borel summability of the WKB solutions.

In the subsequent discussions we will consider not only the usual Borel resummation but also the Borel resummation in a direction $\theta \in \mathbb{R}$ as explained in Section 2.5. Lemma 2.12 shows that the Borel summability of the formal power series $S_{\text {odd }}^{\text {reg }}(z, \eta)$ in the direction $\theta$ is equivalent to the Borel summability of $S_{\text {odd }}^{\mathrm{reg}}\left(z, e^{i \theta} \eta\right)$. Actually, $S_{\text {odd }}^{\text {reg }}\left(z, e^{i \theta} \eta\right)$ coincides with the formal power series (2.19) defined from the Schrödinger equation

$$
\left(\frac{d^{2}}{d z^{2}}-\eta^{2} e^{2 i \theta} Q\left(z, e^{i \theta} \eta\right)\right) \psi=0
$$

(See Lemma 3.8 below.) Therefore, the Borel summability of $S_{\text {odd }}^{\text {reg }}(z, \eta)$ (and of the WKB solutions) in the direction $\theta$ is relevant to the geometry of trajectories of the quadratic differential

$$
\phi_{\theta}=e^{2 i \theta} \phi
$$

Here $\phi$ is the original quadratic differential associated with $\mathcal{L}$. Since the quadratic differential $\phi_{\theta}$ also satisfies Assumption 2.3, trajectories of $\phi_{\theta}$ have the same properties explained in this subsection. Define the Stokes curves in the direction $\theta$ emanating from a turning point $a \in P_{0}$ by

$$
\operatorname{Im}\left(e^{i \theta} \int_{a}^{z} \sqrt{Q_{0}(z)} d z\right)=0
$$

and also define the Stokes graph in the direction $\theta$ by the graph consists of the Stokes curves in the direction $\theta$ and the points in $P$. The Stokes graph in the direction $\theta$ is denoted by $G_{\theta}\left(=G\left(\phi_{\theta}\right)\right)$.

If we vary the direction $\theta$ continuously, the topology of the Stokes graph $G_{\theta}$ changes when a saddle trajectory appears. Let us explain the phenomenon for an example $\phi_{\theta}=e^{2 i \theta}\left(1-z^{2}\right) d z^{\otimes 2}$ defined on $\mathbb{P}^{1}$ (see Figure $3(\mathrm{~d})-(\mathrm{f})$ ). If $\theta \neq 0$ and $|\theta|$ is sufficiently small, there are five Stokes regions; one is a horizontal strip and the other four are half planes (see in Figure 3 (d), (f)). As we vary $\theta$ continuously, the Stokes graph deforms continuously as long as $\theta \neq 0$. However, when $\theta=0$, the horizontal strip disappears from the Stokes graph and the number of Stokes regions becomes four; all Stokes regions are half planes as shown in Figure 3 (e). Moreover, the topologies of the Stokes graphs $G_{\theta}$ for $\theta>0$ and $\theta<0$ are different. A similar change of the topology is also observed when a degenerate saddle trajectory appears (see Figure 3 (g)-(i)). These are typical 


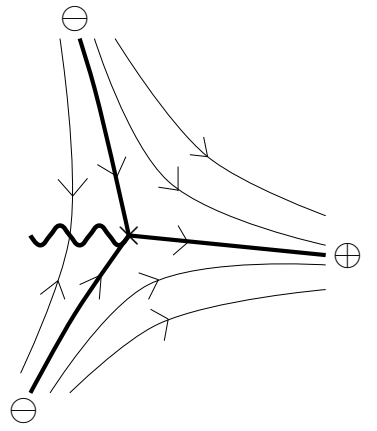

(a) : orientation near a simple turning point.

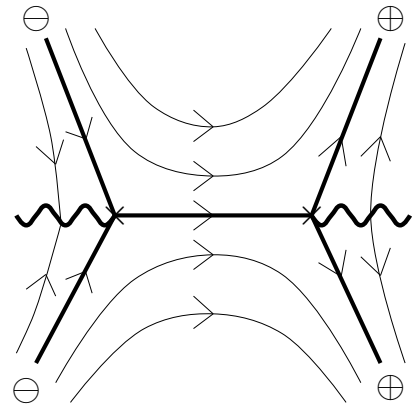

(b) : orientation near a regular saddle trajectory.

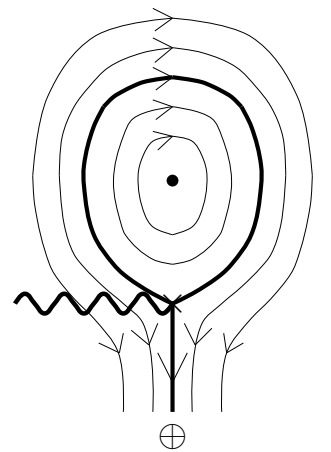

(c) : orientation near a degenerate saddle trajectory.

Figure 7. Examples of orientation of trajectories.

examples of the phenomenon which we call the mutation of Stokes graphs. The mutation of Stokes graphs is the theme of the paper.

\subsection{Orientation of trajectories}

The inverse image of the foliation (2.36) in $\Sigma \backslash P$ by the projection $\pi$ defines a foliation on $\hat{\Sigma} \backslash \pi^{-1} P$. For a trajectory $\beta$ in $\Sigma$, we call each lift of $\beta$ in $\hat{\Sigma}$ by $\pi$ a trajectory in $\hat{\Sigma}$. Since the 1-form defined by $\sqrt{Q_{0}(z)} d z$ is single-valued on $\hat{\Sigma}$, trajectories in $\hat{\Sigma} \backslash \pi^{-1} P$ has the orientation defined by the following rule; the real part of the function $\int^{z} \sqrt{Q_{0}(z)} d z$ increases along the trajectory in the positive direction. Since the covering involution $\tau$ reverses the sign of $\sqrt{Q_{0}(z)}$, the orientation of a trajectories in $\hat{\Sigma}$ is also reversed by $\tau$. Figure 7 depicts examples of the orientation in the first sheet, projected to $\Sigma$ by

$\pi$. The orientation is well-defined on $\hat{\Sigma}$, but its projection has a discontinuity on the branch cut. When we discuss the orientation, we assign the symbols $\oplus$ and $\ominus$ to the asymptotic directions of trajectories entering points of $P_{\infty}$ so that the trajectories with positive directions flows from $\ominus$ to $\oplus$. These signs depend on the choice of the branch cuts and embedding $\iota$, and the covering involution $\tau$ exchanges all signs simultaneously.

\subsection{Borel summability of WKB solutions}

Now we claim an important result concerning with the Borel summability of the WKB solutions for a fixed direction $\theta \in \mathbb{R}$. Note that, setting $\theta=0$ in the following claims, we obtain results for the "usual" Borel summability (Definition 2.10).

Let $\phi$ be the quadratic differential associated with $\mathcal{L}$, and assume that $\phi_{\theta}=e^{2 i \theta} \phi$ has at most one saddle trajectory. Let $G_{\theta}$ be the Stokes graph in the direction $\theta$ in Section 2.6. Take any Stokes region $D$ of $G_{\theta}$. Recall that $D$ must be one of a horizontal strip, a half plane or a degenerate ring domain. Fix a local coordinate $z$ of $\Sigma$ whose domain contains the Stokes region D. Recently, Koike and Schäfke proved the following 
statement which ensures the Borel summability of the formal power series $S_{\text {odd }}^{\text {reg }}(z, \eta)$ on each Stokes region when $\Sigma$ is the Riemann sphere $\mathbb{P}^{1}$.

Theorem $2.17([\overline{\mathrm{KS}}])$. Assume that $\Sigma$ is the Riemann sphere $\mathbb{P}^{1}$, and the coefficients $\left\{Q_{i}(z)\right\}_{i=0}^{n}$ of the potential function of (2.1) are meromorphic functions satisfying Assumption 2.5. Let $G_{\theta}$ and $D$ be as above.

(a). For any fixed $z \in D$, the formal power series $S_{\text {odd }}^{\mathrm{reg}}(z, \eta)$ is Borel summable in the direction $\theta$ as a formal power series in $\eta^{-1}$. The Borel sum of $S_{\text {odd }}^{\text {reg }}(z, \eta)$ becomes holomorphic function of $z$ around the point in question (and also analytic in $\eta$ on $\{\eta \in \mathbb{C}|| \arg \eta-\theta|<\pi / 2,| \eta \mid \gg 1\})$.

(b). Let $p \in P_{\infty}$ be any pole lying on the boundary of $D$. Then, for any fixed $z \in D$, the formal power series defined by the integral

$$
\int_{p}^{z} S_{\text {odd }}^{\mathrm{reg}}(z, \eta) d z
$$

is Borel summable in the direction $\theta$ as a formal power series in $\eta^{-1}$ if the path of the integral (2.41) is contained in $D \cup\{p\}$. The Borel sum becomes holomorphic function of $z$ around the point in question (and also analytic in $\eta$ on $\{\eta \in \mathbb{C}|| \arg \eta-\theta|<\pi / 2,| \eta \mid \gg 1\})$.

Actually, the above claim follows from the results of [KS] and the fact that $S_{\text {odd }}^{\text {reg }}(z, \eta)$ is integrable at each pole (see Proposition [2.8). In [KS the above claim is proved in the case $\theta=0$. The statement for general $\theta$ follows from the result of [KS] together with Lemma 2.12 (see Section 2.6). Although Theorem 2.17 is proved when $\Sigma=\mathbb{P}^{1}$ in $[\mathrm{KS}$, their proof is also applicable to the case when $\Sigma$ is a compact Riemann surface $\Sigma$ since their proof uses only local properties of $\left\{Q_{i}(z)\right\}_{i=0}^{n}$ in each Stokes region and the orders of poles lying on the boundary of $D$. Therefore, we can extend it to the following theorem.

Theorem 2.18. Theorem 2.17 also holds for any compact Riemann surface $\Sigma$.

Since Stokes regions are independent of the choice of the local coordinate, the notion of Borel summability is also independent of the choice. If $z$ lies on a Stokes curve in the direction $\theta$, the trajectory of $\phi_{\theta}$ passing through $z$ flows into a turning point at one end. The proof of Theorem 2.17] by [KS] is not applicable to such a situation.

Next we discuss the Borel summability of the WKB solutions in a fixed direction $\theta \in \mathbb{R}$. Since the WKB solutions are defined by integrating $S_{\text {odd }}(z, \eta) d z$ along a path on the Riemann surface $\hat{\Sigma}$, the Borel summability of the WKB solutions is more delicate than that of $S_{\text {odd }}^{\text {reg }}(z, \eta)$ explained above. To state the criterion of the Borel summability of the WKB solutions proposed by Koike and Schäfke, we introduce the notion of an admissible path. Set $\hat{P}_{0}=\pi^{-1}\left(P_{0}\right), \hat{P}_{\infty}=\pi^{-1}\left(P_{\infty}\right)$ and $\hat{P}=\hat{P}_{0} \cup \hat{P}_{\infty}$.

Definition 2.19. A path $\beta$ on $\hat{\Sigma} \backslash \hat{P}_{0}$ is said to be admissible in the direction $\theta$ if the projection of $\beta$ to $\Sigma$ by $\pi$ either never intersects with the Stokes graph $G_{\theta}$, or intersects with $G_{\theta}$ only at points in $P_{\infty}$. 
Especially, any generic trajectory and any closed trajectory of $\phi_{\theta}$ are admissible in the direction $\theta$. For a given path on $\hat{\Sigma}$ which is not admissible, we may find a decomposition of the path into a finite number of admissible paths as follows.

Lemma 2.20. Let $\beta$ be a path on $\hat{\Sigma} \backslash \hat{P}_{0}$ with end-points $\hat{z}_{1}, \hat{z}_{2} \in \hat{\Sigma} \backslash \hat{P}_{0}$ satisfying the following conditions:

- The end-point $\hat{z}_{1}$ either does not lie on the Stokes graph $G_{\theta}$ or a point in $\hat{P}_{\infty}$. The other end-point $\hat{z}_{2}$ also satisfies the same condition.

- $\beta$ never intersects with a saddle trajectory of $\phi_{\theta}$.

Then, $\beta$ has a decomposition into a finite number of paths $\beta=\beta_{1}+\cdots+\beta_{N}$ in the relative homology group $H_{1}\left(\hat{\Sigma} \backslash \hat{P}_{0}, \hat{P}_{\infty} \cup\left\{\hat{z}_{1}, \hat{z}_{2}\right\} ; \mathbb{Z}\right)$ and each summand $\beta_{i}(1 \leq i \leq N)$ is admissible in the direction $\theta$.

Proof. In the proof we regard a Stokes region as one of its lift in $\hat{\Sigma}$ by the projection $\pi$. Although two Stokes regions in $\hat{\Sigma}$ have the same projection, we distinguish them if they lie on different sheets of $\hat{\Sigma}$. Moreover, since we only consider the Stokes graph for a fixed $\theta$, we omit "in the direction $\theta$ " for simplicity.

Since any point in a Stokes region and a point in $\hat{P}_{\infty}$ which lies on the boundary of the Stokes region can be connected by an admissible path, in the proof we may assume that $\beta$ never passes through a point in $\hat{P}_{\infty}$ (i.e., $\beta$ is contained in $\hat{\Sigma} \backslash \hat{P}$ ) without loss of generality. Especially, we may assume that $\hat{z}_{1}, \hat{z}_{2} \notin \hat{P}_{\infty}$. If $\hat{z}_{1}, \hat{z}_{2}$ and the path $\beta$ are contained in the same Stokes region, $\beta$ is admissible by definition. Therefore, it suffices to consider the case that $\hat{z}_{1}$ and $\hat{z}_{2}$ are contained in different Stokes regions and the path $\beta$ connects them crossing finitely many Stokes curves which are not saddle trajectories. We may also assume that the Stokes regions containing $\hat{z}_{1}$ or $\hat{z}_{2}$ are not degenerate ring domains (otherwise a path $\beta$ satisfying the assumption never exists).

Let us consider the case that $\beta$ intersect with a Stokes curve just once. Since the Stokes curve is not a saddle trajectory, it must be a separating trajectory by Lemma 2.15. That is, the Stokes curve connects a point $a \in \hat{P}_{0}$ and a point $p \in \hat{P}_{\infty}$. Therefore, we can decompose $\beta$ into a sum of two paths $\beta_{1}+\beta_{2}$ in the relative homology group, where $\beta_{1}$ (resp., $\beta_{2}$ ) connects $\hat{z}_{1}$ (resp., $\hat{z}_{2}$ ) and $p$ as indicated in Figure 8 . Here we can take the path $\beta_{1}$ (resp., $\beta_{2}$ ) to be admissible since the point $p$ lies on the boundary of the Stokes region containing $\hat{z}_{1}$ (resp., $\hat{z}_{2}$ ).

Any path $\beta$ in $\hat{\Sigma} \backslash \hat{P}$ can be written by the sum of a finite number of paths whose each summand intersect with the Stokes curves just once. Therefore, applying the decomposition as in Figure 8 to each summand, we can find a desired decomposition of $\beta$ by admissible paths.

Then, a criterion of the Borel summability of the WKB solutions proposed by Koike and Schäfke is stated as follows.

Corollary 2.21 ([KS]). (a). Let $\beta$ be a path on $\hat{\Sigma} \backslash \hat{P}_{0}$ with end-points $\hat{z}_{1}, \hat{z}_{2} \in \hat{\Sigma} \backslash \hat{P}_{0}$ satisfying the same assumption in Lemma 2.20. Then, the formal power series $\int_{\beta} S_{\mathrm{odd}}^{\mathrm{reg}}(z, \eta) d z$ is Borel summable in the direction $\theta$. 


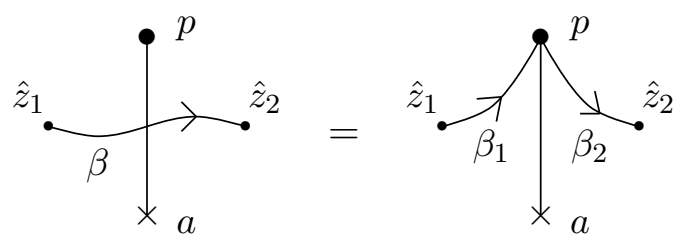

Figure 8. An example of decomposition of a path which intersects with the Stokes curves just once.

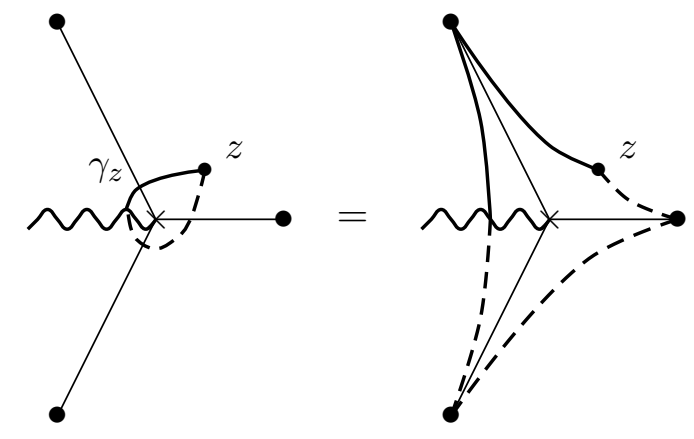

Figure 9. Decomposition of $\gamma_{z}$ into admissible paths.

(b). If the Stokes graph $G_{\theta}$ is saddle-free, then the WKB solutions which are normalized as (2.26) and (2.28) are Borel summable in the direction $\theta$ at any point $z$ in each Stokes region. The Borel sums of the WKB solutions give analytic solutions of (2.1) on each Stokes region (which is also analytic in $\eta$ on a domain $\{\eta \in \mathbb{C}|| \arg \eta-\theta \mid<$ $\pi / 2,|\eta| \gg 1\})$.

Proof. Theorem 2.17 (b) ensures that a formal power series defined by integrating $S_{\text {odd }}^{\text {reg }}(z, \eta)$ along an admissible path is Borel summable in the direction $\theta$. Therefore, the first claim (a) follows from Lemma 2.20.

Let us show the claim (b). When the Stokes graph is saddle-free, any path $\beta$ on $\hat{\Sigma} \backslash \hat{P}_{0}$ can be decomposed into admissible paths by Lemma 2.20. For example, if $z$ lies on a Stokes region, then the path $\gamma_{z}$ (see Figure 2) which determines the WKB solutions (2.26) is decomposed into admissible paths as depicted in Figure 9, Therefore the integral $\int_{\gamma_{z}} S_{\text {odd }}^{\text {reg }}(z, \eta) d z$ is Borel summable in the direction $\theta$ by Theorem 2.17 (b). The Borel summability of the WKB solutions (2.26) follows from (c) in Proposition 2.11. The Borel summability of the WKB solutions (2.28) can be shown similarly.

Remark 2.22. Suppose that the Stokes graph has a saddle trajectory. Even if the point $z$ does not lie on the Stokes graph, the path $\gamma_{z}$ in Figure 2 can not be decomposed into admissible paths when $\gamma_{z}$ intersects with the saddle trajectory. Therefore, we can not expect the Borel summability for the WKB solutions in general when a saddle trajectory appears in the Stokes graph. 
The above statements guarantee the Borel summability of $S_{\text {odd }}^{\text {reg }}(z, \eta)$ and the WKB solutions in a fixed direction $\theta$. As is explained in Section 2.5, the rotation of the direction $\theta$ may break the Borel summability of the WKB solutions. The following claim gives an criterion for the invariance of the Borel sum under a rotation of $\theta$.

Proposition 2.23. Let $\beta$ be a path on $\hat{\Sigma} \backslash \hat{P}_{0}$ with end-points $\hat{z}_{1}, \hat{z}_{2} \in \hat{\Sigma} \backslash \hat{P}_{0}$. Suppose that there exist real numbers $\theta_{1}, \theta_{2}$ with $\theta_{1}<\theta_{2}$ such that the following conditions hold.

- The quadratic differential $\phi_{\theta}$ has at most one saddle trajectory for any $\theta_{1} \leq \theta \leq \theta_{2}$.

- The end-point $\hat{z}_{1}$ either does not lie on the Stokes graphs $G_{\theta}$ for any $\theta_{1} \leq \theta \leq \theta_{2}$ or is a point in $\hat{P}_{\infty}$. The other end-point $\hat{z}_{2}$ also satisfies the same condition.

- The path $\beta$ never touches with a saddle trajectory of $\phi_{\theta}$ for any $\theta_{1} \leq \theta \leq \theta_{2}$.

Then, the Borel sums of the formal power series $f(\eta)=\int_{\beta} S_{\text {odd }}^{\text {reg }}(z, \eta) d z$ in the direction $\theta_{1}$ and $\theta_{2}$ coincide. That is, the following equality holds as analytic functions of $\eta$ defined on a domain containing $\left\{\eta \in \mathbb{C}\left|\theta_{1}-\pi / 2<\arg \eta<\theta_{2}+\pi / 2,\right| \eta \mid \gg 1\right\}$ :

$$
\mathcal{S}_{\theta_{1}}[f](\eta)=\mathcal{S}_{\theta_{2}}[f](\eta)
$$

Proof. Since $\beta$ satisfies the assumption of Corollary 2.21 (a) for any $\theta$ satisfying $\theta_{1} \leq \theta \leq \theta_{2}$, the formal power series $f(\eta)$ is Borel summable in all directions $\theta_{1} \leq \theta \leq \theta_{2}$. That means that the Borel transform $f_{B}(y)$ of $f(\eta)$ does not have singular points in a domain containing the sector $\left\{y=r e^{-i \theta} \mid r \geq 0, \theta_{1} \leq \theta \leq \theta_{2}\right\}$, and has an exponential growth near $y=\infty$ (see Definition 2.10). Hence, the Laplace integrals (2.32) give the same analytic function of $\eta$ for all $\theta_{1} \leq \theta \leq \theta_{2}$. Thus we obtain (2.42).

Remark 2.24. The "resurgence property" (i.e., the endlessly continuability of the Borel transform), which is stronger than the Borel summability, was claimed by Ecalle in [Eca84, but not all details are clear (see [DP99, Comment in Section 1.2]). Still currently there are many contributions to warrant the resurgence property of the WKB solutions; e.g., Get09, Get11, GT11. The case where the potential function is entire on $\mathbb{C}$ is discussed in these works.

\subsection{Connection formula for WKB solutions}

Corollary 2.21 (b) ensures that, if the Stokes graph $G_{\theta}$ in a fixed direction $\theta$ is saddlefree, then the WKB solutions are Borel summable in the direction $\theta$ on each Stokes region of $G_{\theta}$. Here we show an explicit and simple connection formula between the Borel sums of the WKB solutions defined on adjacent Stokes regions found by Voros Vor83. (In this subsection we do not consider the rotation of the direction $\theta$. The following statements hold for any fixed $\theta$, if the Stokes graph $G_{\theta}$ is saddle-free.)

Here we specify the situation to state the connection formula. Assume that the Stokes graph $G_{\theta}$ is saddle-free. Let $a \in P_{0}$ be a simple turning point, and suppose that two Stokes regions $D_{1}$ and $D_{2}$ have a common boundary $C$ which is a Stokes curve emanating from $a$, and $D_{2}$ comes next to $D_{1}$ in the counter-clockwise direction with the 


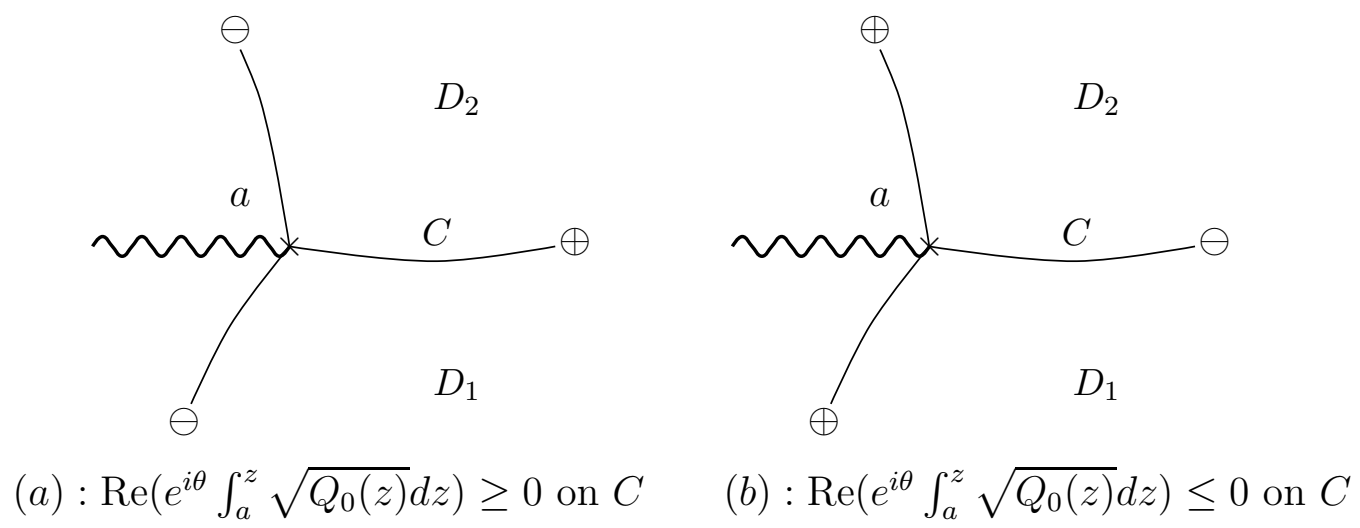

Figure 10. Two possibilities of assignment of sign.

reference point $a$. Take appropriate branch cuts so that $C$ does not cross any branch cut. Then we have two possibilities (a) and (b) shown in Figure 10 for the sign of the other end-point of $C$ than $a$. For each case, the connection formula is formulated as follows.

Theorem 2.25 ([Vor83, Section 6], [AKT91, Section 2]). Suppose that the Stokes graph $G_{\theta}$ is saddle-free. Let $a \in P_{0}, C, D_{1}$ and $D_{2}$ be as above and

$$
\psi_{ \pm}(z, \eta)=\frac{1}{\sqrt{S_{\text {odd }}(z, \eta)}} \exp \left( \pm \int_{a}^{z} S_{\text {odd }}(z, \eta) d z\right)
$$

be the WKB solutions normalized at the turning point a as defined in (2.26). Denote by $\Psi_{ \pm}^{D_{j}} \quad(j=1,2)$ the Borel sum of $\psi_{ \pm}$on the Stokes region $D_{j}(j=1,2)$. Then, the analytic continuation of $\Psi_{ \pm}^{D_{1}}$ to $D_{2}$ across the Stokes curve $C$ satisfy the following equalities:

$$
\begin{aligned}
& \left\{\begin{array}{l}
\Psi_{+}^{D_{1}}=\Psi_{+}^{D_{2}}+i \Psi_{-}^{D_{2}}, \\
\Psi_{-}^{D_{1}}=\Psi_{-}^{D_{2}},
\end{array}\right. \\
& \left\{\begin{array}{l}
\Psi_{+}^{D_{1}}=\Psi_{+}^{D_{2}}, \\
\Psi_{-}^{D_{1}}=\Psi_{-}^{D_{2}}+i \Psi_{+}^{D_{2}},
\end{array}\right.
\end{aligned}
$$

Here $i$ appearing in the formula is the imaginary unit $\sqrt{-1}$.

Remark 2.26. Theorem 2.25 is proved by [Vor83] and [AKT91] in the case that $\Sigma=\mathbb{P}^{1}$. Since the proof of [AKT91, Appendix A.2] is based only on local properties of the WKB solutions near a simple turning point, the same discussion is applicable to a general compact Riemann surface $\Sigma$. Therefore, together with Theorem 2.18 , Theorem 2.25] is valid when $\Sigma$ is a general compact Riemann surface.

Remark 2.27. Theorem 2.25 gives connection formulas which describe the analytic continuation of the Borel sums of WKB solutions on the $z$-plane. On the other hand, 
the formulas indeed describe the Stokes phenomenon (for a large $\eta$ ) occurring to WKB solutions since they are derived through the analysis of the singularity of the Borel transforms of WKB solutions (see [KT05]). That is, we can reformulate Theorem 2.25 in an alternative manner as follows: Take any point $z \in \hat{\Sigma} \backslash \hat{P}$, and suppose that there exists a direction $\theta_{0}$ and a sufficiently small number $\varepsilon>0$ satisfying

- Stokes graphs in any direction $\theta$ satisfying $\theta_{0}-\varepsilon \leq \theta \leq \theta_{0}+\varepsilon$ is saddle-free,

- the point $z$ lies on a Stokes curve $C$ in the direction $\theta_{0}$, and does not lie on the Stokes graph in any direction $\theta$ satisfying $\theta_{0}-\varepsilon \leq \theta<\theta_{0}$ or $\theta_{0}<\theta \leq \theta_{0}+\varepsilon$.

Let $\psi_{ \pm}$be the WKB solution normalized at the turning point from where the Stokes curve $C$ emanates, and denote by $\Psi_{ \pm}^{\left(\theta_{0}-\varepsilon\right)}$ (resp., $\Psi_{ \pm}^{\left(\theta_{0}+\varepsilon\right)}$ ) the Borel sum of $\psi_{ \pm}$in the direction $\theta_{0}-\varepsilon$ (resp., $\theta_{0}+\varepsilon$ ) Then, the following relation holds in a neighborhood of $z$.

$$
\begin{aligned}
& \left\{\begin{array}{l}
\Psi_{+}^{\left(\theta_{0}-\varepsilon\right)}=\Psi_{+}^{\left(\theta_{0}+\varepsilon\right)}+i \Psi_{-}^{\left(\theta_{0}+\varepsilon\right)}, \\
\Psi_{-}^{\left(\theta_{0}-\varepsilon\right)}=\Psi_{-}^{\left(\theta_{0}+\varepsilon\right)},
\end{array}\right. \\
& \left\{\begin{array}{l}
\Psi_{+}^{\left(\theta_{0}-\varepsilon\right)}=\Psi_{+}^{\left(\theta_{0}+\varepsilon\right)}, \\
\Psi_{-}^{\left(\theta_{0}-\varepsilon\right)}=\Psi_{-}^{\left(\theta_{0}+\varepsilon\right)}+i \Psi_{+}^{\left(\theta_{0}+\varepsilon\right)},
\end{array} \text { if the sign of the end point of } C \text { is } \ominus .\right.
\end{aligned}
$$

Thus, the Stokes phenomenon occurring to $\psi_{ \pm}$is described by the completely same formulas in Theorem 2.25. We use this point of view in Appendix B.

The connection formula in Theorem 2.25 is quite effective for the global problems of differential equations. For example, if $\Sigma=\mathbb{P}^{1}$ and the equation $\mathcal{L}$ is Fuchsian (i.e., all poles of $\phi$ are order 2) with a saddle-free Stokes graph, then the monodromy group of $\mathcal{L}$ can be expressed by the following quantities ([KT05, Theorem 3.5]):

(i) characteristic exponents at regular singular points,

(ii) the Borel sum of contour integrals of $S_{\text {odd }}(z, \eta) d z$ along cycles in $\hat{\Sigma} \backslash \hat{P}$.

In [KT05, Section 3.1] a recipe to obtain an explicit expression of the monodromy group is given. Contour integrals of $S_{\text {odd }}(z, \eta) d z$ appear when we use the connection formulas (2.43) and (2.44) iteratively.

\section{Voros symbols and Stokes automorphisms}

In this section we introduce an important notion in the exact WKB analysis, called the Voros symbols. We discuss the jump property of the Voros symbols caused by the Stokes phenomenon relevant to the appearance of saddle trajectories in the Stokes graph.

\subsection{Homology groups and Voros symbols}

Let us consider the homology group $H_{1}(\hat{\Sigma} \backslash \hat{P})=H_{1}(\hat{\Sigma} \backslash \hat{P} ; \mathbb{Z})$ and the relative homology group $H_{1}\left(\hat{\Sigma} \backslash \hat{P}_{0}, \hat{P}_{\infty}\right)=H_{1}\left(\hat{\Sigma} \backslash \hat{P}_{0}, \hat{P}_{\infty} ; \mathbb{Z}\right)$. In what follows we call elements of $H_{1}(\hat{\Sigma} \backslash \hat{P})$ 
and $H_{1}\left(\hat{\Sigma} \backslash P_{0}, \hat{P}_{\infty}\right)$ as cycles and paths, respectively, to distinguish them. By the Lefschetz duality there exists a bilinear form

$$
\langle,\rangle: H_{1}(\hat{\Sigma} \backslash \hat{P}) \times H_{1}\left(\hat{\Sigma} \backslash \hat{P}_{0}, \hat{P}_{\infty}\right) \rightarrow \mathbb{Z}
$$

on these homology groups given by the intersection number of cycles and paths. The intersection number depends on the orientations of cycles and paths, and we normalize the bilinear form as $\langle x$-axis, $y$-axis $\rangle=+1$. It also induces a bilinear form

$$
(,): H_{1}(\hat{\Sigma} \backslash \hat{P}) \times H_{1}(\hat{\Sigma} \backslash \hat{P}) \rightarrow \mathbb{Z} .
$$

We call both these bilinear forms intersection forms.

Here we introduce the notion of the Voros symbols, which are the main objects in this paper.

Definition 3.1 ([DDP93, Section 1.2]). - Let $\beta \in H_{1}\left(\hat{\Sigma} \backslash \hat{P}_{0}, \hat{P}_{\infty}\right)$ be a path. The formal power series $e^{W_{\beta}}$ is called the Voros symbol for the path $\beta$. Here $W_{\beta}=W_{\beta}(\eta)$ is the formal power series defined by the integral

$$
W_{\beta}(\eta)=\int_{\beta} S_{\mathrm{odd}}^{\mathrm{reg}}(z, \eta) d z .
$$

- Let $\gamma \in H_{1}(\hat{\Sigma} \backslash \hat{P})$ be a cycle. The formal series $e^{V_{\gamma}}$ is called the Voros symbol for the cycle $\gamma$. Here $V_{\gamma}=V_{\gamma}(\eta)$ is the formal series defined by the integral

$$
V_{\gamma}(\eta)=\oint_{\gamma} S_{\text {odd }}(z, \eta) d z .
$$

Remark 3.2. The formal series $W_{\beta}$ in (3.3) (resp., $V_{\gamma}$ in (3.4)) is called the Voros coefficient for the path $\beta$ (resp., for the cycle $\gamma$ ). The Voros coefficients for paths in $H_{1}\left(\hat{\Sigma} \backslash \hat{P}_{0}, \hat{P}_{\infty}\right)$ attract attention recently (e.g., [Tak08], [AT13]).

The Voros symbols $e^{W_{\beta}}$ for $\beta \in H_{1}\left(\hat{\Sigma} \backslash \hat{P}_{0}, \hat{P}_{\infty}\right)$ are formal power series without a exponential factor since $S_{\text {odd }}^{\text {reg }}(z, \eta)$ is a formal power series. On the other hand, the Voros symbols $e^{V_{\gamma}}$ for $\gamma \in H_{1}(\hat{\Sigma} \backslash \hat{P})$ are formal series with the exponential factors $\exp \left(\eta v_{\gamma}\right)$, where

$$
v_{\gamma}=\oint_{\gamma} \sqrt{Q_{0}(z)} d z
$$

As mentioned in Section 2.9, the Voros symbols appear in the expression of monodromy group of the equation (2.1) (see Section 2.9). They are Borel summable (in the direction $\theta=0$ ) if the paths of the integrals in (3.3) and (3.4) do not intersect with a saddle trajectory of $\phi$ by Corollary 2.21. The appearance of a saddle trajectory breaks the Borel summability, and cause the Stokes phenomenon as explained in Section 2.5. That is, if a saddle trajectory appears, the Borel sums of a Voros symbol in the directions $\pm \delta$ are different in general for a sufficiently small $\delta>0$. As noted in Section 2.6, the Stokes graph mutates when a saddle trajectory appears. The rest of this section is devoted to analyze the Stokes phenomenon occurring to the Voros symbols under the mutation of Stokes graphs. 


\subsection{Saddle class associated with saddle trajectory}

Suppose that the Stokes graph $G_{0}=G(\phi)$ has a regular or degenerate saddle trajectory $\ell_{0}$. Recall that a regular saddle trajectory connects two different zeros of $\phi$, while a degenerate saddle trajectory forms a closed loop around a double pole of $\phi$ (see Section 2.6). Then, there exists a cycle $\gamma_{0} \in H_{1}(\hat{\Sigma} \backslash \hat{P})$ whose projection on $\Sigma$ by $\pi$ surrounds $\ell_{0}$ as in Figure 11, and its orientation is given so that

$$
v_{\gamma_{0}}=\oint_{\gamma_{0}} \sqrt{Q_{0}(z)} d z<0
$$

(See Section 2.7 for the rule of the assignment of signs.) Note that, if a cycle $\gamma_{0}$ satisfies the above conditions, then the cycle $-\gamma_{0}^{*}$ also satisfies the same conditions. (Here $\gamma_{0}^{*}$ is the image of $\gamma_{0}$ by the covering involution $\tau$.) We choose any of the two cycles, and call the resulting homology class $\gamma_{0} \in H_{1}(\hat{\Sigma} \backslash \hat{P})$ the saddle class associated with the saddle trajectory $\ell_{0}$, following [BS13, Section 3.6]. Note that "the Voros symbol for the saddle class" is well-defined because

$$
\oint_{\gamma} S_{\text {odd }}(z, \eta) d z=\oint_{-\gamma^{*}} S_{\text {odd }}(z, \eta) d z
$$

holds for any cycle $\gamma$ due to the anti-invariant property (2.25) of $S_{\text {odd }}(z, \eta)$.

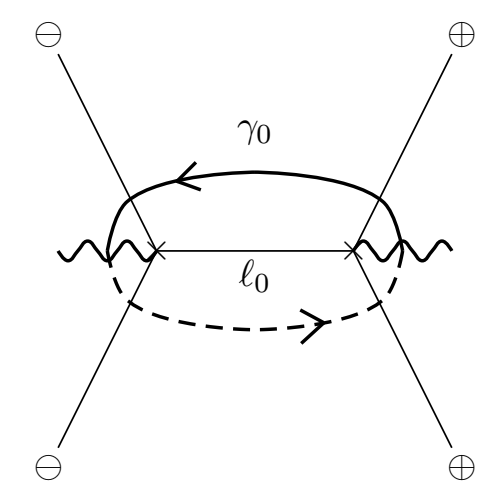

$\ell_{0}$ : a regular saddle trajectory

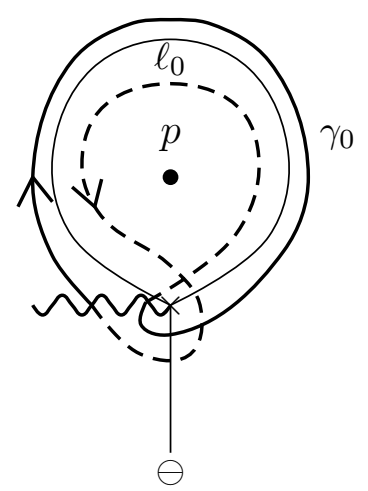

$\ell_{0}:$ a degenerate saddle trajectory

Figure 11. Saddle trajectories and the associated saddle classes.

\subsection{Saddle reduction}

Suppose that the Stokes graph $G_{0}=G(\phi)$ has a unique regular or degenerate saddle trajectory $\ell_{0}$. Then, as in BS13, Section 5 and Section 10.3], there exists $r>0$ such that for all $0<\delta \leq r$ the quadratic differentials $\phi_{ \pm \delta}=e^{ \pm 2 i \delta} \phi$ are saddle-free. We call $G_{ \pm \delta}$ saddle reductions of $G_{0}$. The topology of the Stokes graph $G_{+\delta}=G\left(\phi_{+\delta}\right)$ (resp., $\left.G_{-\delta}=G\left(\phi_{-\delta}\right)\right)$ does not change as long as $0<\delta \leq r$ since $\phi_{+\delta}$ (resp., $\left.\phi_{-\delta}\right)$ is saddle-free for all $0<\delta \leq r$. However, varying $\delta$ across 0 , the topology of the Stokes graph changes as explained in Section 2.6. We say that $G_{-\delta}$ and $G_{+\delta}$ are related by a flip (resp., pop) if 

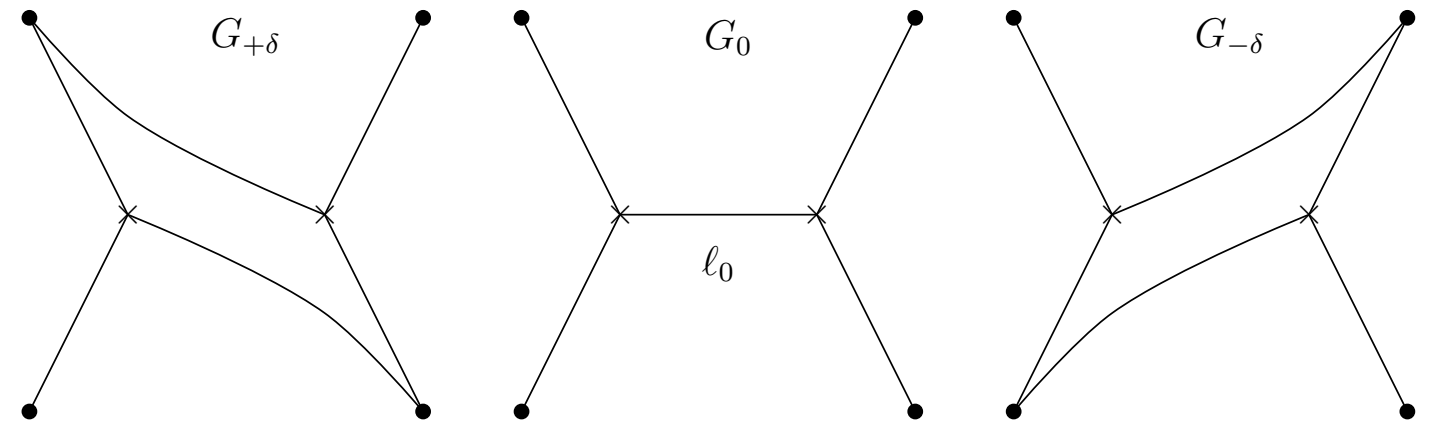

(a) Saddle reduction of regular saddle trajectory and flip.
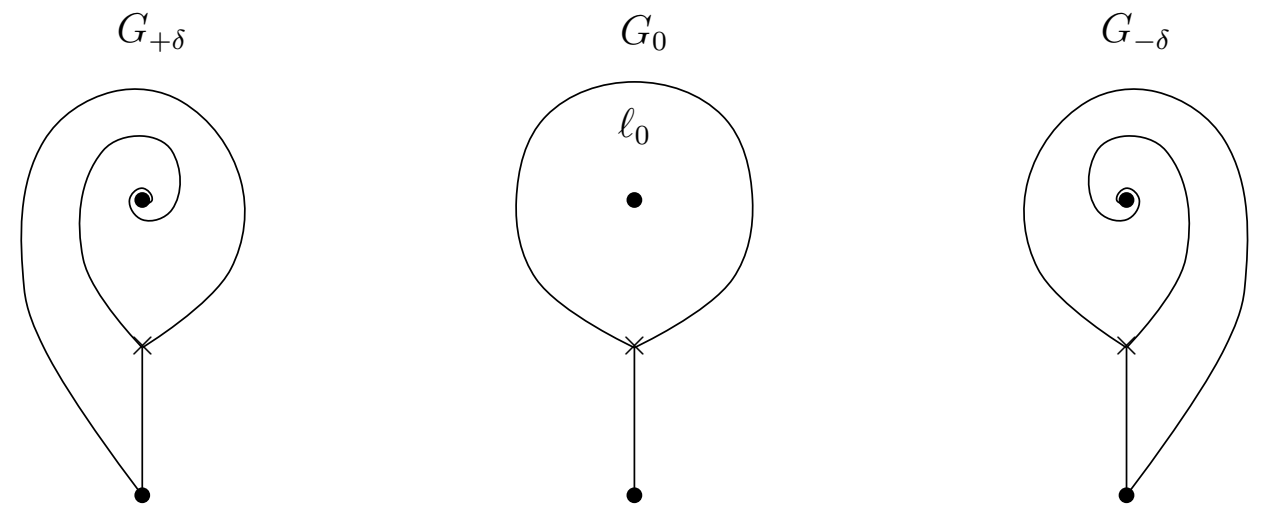

(b) Saddle reduction of degenerate saddle trajectory and pop.

Figure 12. Reduction of saddle trajectories. Figures describe a part of Stokes graphs.

they give saddle reductions of a regular (resp., degenerate) saddle trajectory (see Figure 12).

Since the Stokes graphs $G_{ \pm \delta}$ are saddle-free, the Voros symbols are Borel summable in any direction $\pm \delta$ with $0<\delta \leq r$ by Corollary 2.21. Furthermore, we can show the following.

Lemma 3.3. Suppose that the Stokes graph $G_{0}$ has a unique saddle trajectory. Then, there exists a sufficiently small $r>0$ such that the following equalities hold as analytic functions of $\eta$ for any $0<\delta_{1}, \delta_{2} \leq r$ :

$$
\begin{array}{ll}
\mathcal{S}_{+\delta_{1}}\left[e^{W_{\beta}}\right](\eta)=\mathcal{S}_{+\delta_{2}}\left[e^{W_{\beta}}\right](\eta), & \mathcal{S}_{+\delta_{1}}\left[e^{V_{\gamma}}\right](\eta)=\mathcal{S}_{+\delta_{2}}\left[e^{V_{\gamma}}\right](\eta), \\
\mathcal{S}_{-\delta_{1}}\left[e^{W_{\beta}}\right](\eta)=\mathcal{S}_{-\delta_{2}}\left[e^{W_{\beta}}\right](\eta), & \mathcal{S}_{-\delta_{1}}\left[e^{V_{\gamma}}\right](\eta)=\mathcal{S}_{-\delta_{2}}\left[e^{V_{\gamma}}\right](\eta) .
\end{array}
$$

Here $\beta \in H_{1}\left(\hat{\Sigma} \backslash \hat{P}_{0}, \hat{P}_{\infty}\right)$ and $\gamma \in H_{1}(\hat{\Sigma} \backslash \hat{P})$ are any path and cycle, respectively.

Proof. Note that any path $\beta \in H_{1}\left(\hat{\Sigma} \backslash \hat{P}_{0}, \hat{P}_{\infty}\right)$ or any cycle $\gamma \in H_{1}(\hat{\Sigma} \backslash \hat{P})$ is decomposed into the sum of a finite number of paths whose end-points are contained in $\hat{P}_{\infty}$ in the relative homology group $H_{1}\left(\hat{\Sigma} \backslash \hat{P}_{0}, \hat{P}_{\infty}\right)$ (see Figure 13). Therefore, it suffices to show the equalities (3.8) and (3.9) for any $\beta \in H_{1}\left(\hat{\Sigma} \backslash \hat{P}_{0}, \hat{P}_{\infty}\right)$ whose end-points are contained in $\hat{P}_{\infty}$. Take any such a path $\beta$, and fix a sufficiently small $r>0$ so that the Stokes graphs $G_{ \pm \delta}$ are saddle-free for all $0<\delta \leq r$. Then, the path $\beta \in H_{1}\left(\hat{\Sigma} \backslash \hat{P}_{0}, \hat{P}_{\infty}\right)$ never 
touches with saddle trajectory of $\phi_{ \pm \delta}$ for all $0<\delta \leq r$. Therefore, since $\beta$ satisfies the assumption of Proposition 2.23, the equalities (3.8) and (3.9) follows form (2.42).
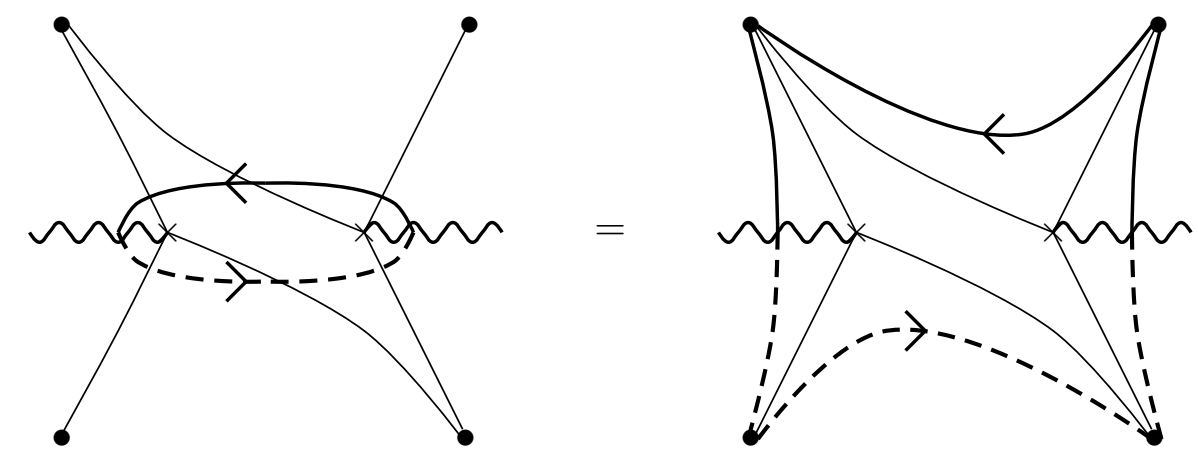

Figure 13. An example of decomposition of a cycles into the sum of paths whose end-points are contained in $\hat{P}_{\infty}$.

Define $\mathcal{S}_{ \pm}\left[e^{W_{\beta}}\right]=\mathcal{S}_{ \pm}\left[e^{W_{\beta}}\right](\eta)$ (resp., $\mathcal{S}_{ \pm}\left[e^{V_{\gamma}}\right]=\mathcal{S}_{ \pm}\left[e^{V_{\gamma}}\right](\eta)$ ) by the Borel sum $\mathcal{S}_{ \pm \delta}\left[e^{W_{\beta}}\right](\eta)$ (resp., $\mathcal{S}_{ \pm \delta}\left[e^{V_{\gamma}}\right](\eta)$ ) of the Voros symbol for a path $\beta \in H_{1}\left(\hat{\Sigma} \backslash \hat{P}_{0}, \hat{P}_{\infty}\right)$ (resp., for a cycle $\gamma \in H_{1}(\hat{\Sigma} \backslash \hat{P})$ ) for a sufficiently small $\delta>0$. Due to Lemma [3.3, $\mathcal{S}_{ \pm}\left[e^{W_{\beta}}\right]$ and $\mathcal{S}_{ \pm}\left[e^{V_{\gamma}}\right]$ are well-defined. As explained in Section [2.5, the Borel sums $\mathcal{S}_{ \pm}\left[e^{W_{\beta}}\right]$ and $\mathcal{S}_{ \pm}\left[e^{V_{\gamma}}\right]$ are analytic in $\eta$ on a domain containing $\{\eta \in \mathbb{R} \mid \eta \gg 1\}$. In the rest of this section we will describe the relationship between $\mathcal{S}_{+}\left[e^{W_{\beta}}\right]$ (resp., $\mathcal{S}_{+}\left[e^{V_{\gamma}}\right]$ ) and $\mathcal{S}_{-}\left[e^{W_{\beta}}\right]$ (resp., $\left.\mathcal{S}_{-}\left[e^{V_{\gamma}}\right]\right)$; that is, the formulas describing the Stokes phenomenon occurring to the Voros symbols.

\subsection{Jump formula and Stokes automorphism for regular saddle trajectory}

Here we specify the situation to state Theorem 3.4 below. Suppose that the Stokes graph $G_{0}=G(\phi)$ has a unique regular saddle trajectory $\ell_{0}$ with the associated saddle class $\gamma_{0} \in H_{1}(\hat{\Sigma} \backslash \hat{P})$. Let $G_{ \pm \delta}=G\left(\phi_{ \pm \delta}\right)$ be saddle reductions of $G_{0}$ for a sufficiently small $\delta>0$ as in (a) of Figure 12 (i.e., $G_{-\delta}$ and $G_{+\delta}$ is related by a flip). Then, the Stokes phenomenon occurring to the Voros symbols are described explicitly by the following "jump formula".

Theorem 3.4 ([DDP93, Section 3]). The Borel sums $\mathcal{S}_{ \pm}\left[e^{W_{\beta}}\right]$ and $\mathcal{S}_{ \pm}\left[e^{V_{\gamma}}\right]$ for any $\beta \in H_{1}\left(\hat{\Sigma} \backslash \hat{P}_{0}, \hat{P}_{\infty}\right)$ and any $\gamma \in H_{1}(\hat{\Sigma} \backslash \hat{P})$ satisfy the following equalities as analytic functions of $\eta$ on a domain containing $\{\eta \in \mathbb{R} \mid \eta \gg 1\}$ :

$$
\begin{aligned}
\mathcal{S}_{-}\left[e^{W_{\beta}}\right] & =\mathcal{S}_{+}\left[e^{W_{\beta}}\right]\left(1+\mathcal{S}_{+}\left[e^{V_{\gamma_{0}}}\right]\right)^{-\left\langle\gamma_{0}, \beta\right\rangle}, \\
\mathcal{S}_{-}\left[e^{V_{\gamma}}\right] & =\mathcal{S}_{+}\left[e^{V_{\gamma}}\right]\left(1+\mathcal{S}_{+}\left[e^{V_{\gamma_{0}}}\right]\right)^{-\left(\gamma_{0}, \gamma\right)} .
\end{aligned}
$$

Remark 3.5. Originally, Theorem 3.4 is proved in [DDP93, Section 3] for the case that the potential $Q(z, \eta)=Q_{0}(z)$ is independent of $\eta$ and is a polynomial in $z$. Since the Borel summability of the WKB solutions are established in [KS] (see Theorem 2.17 and 
2.18), the proof of [DDP93] is also valid for general cases. For a convenience of readers, we briefly recall the sketch of the proof of Theorem 3.4 in Appendix A.

The formula (3.10) in fact describes the Stokes phenomenon for the Voros symbols relevant to the flip of the Stokes graph. The exponentially small difference between the Borel sums of Voros symbols are explicitly given in (3.10). Note that the Borel sum $\mathcal{S}_{ \pm}\left[e^{V_{\gamma_{0}}}\right]$ is exponentially small for a sufficiently large $\eta \gg 1$ because it is asymptotically expanded to the formal series $e^{V_{\gamma_{0}}}$ as $\eta \rightarrow+\infty$ whose exponential factor $e^{\eta v_{\gamma_{0}}}$ is exponentially small due to the orientation (3.6) of the saddle class $\gamma_{0}$.

In DDP93] the formula (3.10) is stated in a different manner. Let $\mathbb{V}=\mathbb{V}(Q(z, \eta))$ be the field of the rational functions generated by the Voros symbols $e^{W_{\beta}}$ and $e^{V_{\gamma}}$, which we call the Voros field for a potential $Q(z, \eta)$. Define a field automorphism $\mathfrak{S}_{\gamma_{0}}: \mathbb{V} \rightarrow \mathbb{V}$ by

$$
\mathfrak{S}_{\gamma_{0}}: \begin{cases}e^{W_{\beta}} \mapsto e^{W_{\beta}}\left(1+e^{V_{\gamma_{0}}}\right)^{-\left\langle\gamma_{0}, \beta\right\rangle} & \left(\beta \in H_{1}\left(\hat{\Sigma} \backslash \hat{P}_{0}, \hat{P}_{\infty}\right)\right), \\ e^{V_{\gamma}} \mapsto e^{V_{\gamma}}\left(1+e^{V_{\gamma_{0}}}\right)^{-\left(\gamma_{0}, \gamma\right)} & \left(\gamma \in H_{1}(\hat{\Sigma} \backslash \hat{P})\right) .\end{cases}
$$

The equalities (3.10) implies that $\mathfrak{S}_{\gamma_{0}}$ satisfies

$$
\mathcal{S}_{-}=\mathcal{S}_{+} \circ \mathfrak{S}_{\gamma_{0}}
$$

Here $\mathcal{S}_{ \pm}$is the Borel summation operator $\mathcal{S}_{ \pm \delta}$ for a sufficiently small $\delta>0$. To be precise, the map $\mathcal{S}_{ \pm}$in Definition 2.10 is not defined for sums of Voros symbols with different exponential factors. Here we extend it to the map from $\mathbb{V}$ to a space of analytic functions of $\eta$ so that $\mathcal{S}_{ \pm}$commutes with the operations addition, multiplication, and division. In view of (3.12), the map $\mathfrak{S}_{\gamma_{0}}$ measures the difference between the Borel sums of Voros symbols for different directions. The map $\mathfrak{S}_{\gamma_{0}}$ is called the Stokes automorphism for the saddle class $\gamma_{0}$ associated with a regular saddle trajectory $\ell_{0}$ (see [DP99, Section $0.4])$.

We call the formulas (3.10) and (3.11) the DDP (Delabaere-Dillinger-Pham) formula. Later in Section 7 we will reformulate the DDP formula in view of cluster algebras theory. Furthermore, we apply this formulation to study identities of Stokes automorphisms.

Remark 3.6. The DDP formula resembles to the Kontsevich-Soibelman transformation in GMN13, where the counterpart of the Voros symbols are the Fock-Goncharov coordinates of the moduli space of the flat connections associated with a Hitchin system of rank 2. In their context, a quadratic differential appears as the image of the Hitchin fibration, and its saddle trajectories capture BPS states in a four dimensional field theory.

\subsection{Jump formula and Stokes automorphism for degenerate saddle trajectory}

Similarly to regular saddle trajectories, degenerate saddle trajectories also cause the Stokes phenomenon for the Voros symbols. This subsection is devoted to the description 
of the formula for the Voros symbols describing the Stokes phenomenon. Suppose that the Stokes graph $G_{0}=G(\phi)$ has a unique degenerate saddle trajectory $\ell_{0}$ with the associated saddle class $\gamma_{0} \in H_{1}(\hat{\Sigma} \backslash \hat{P})$. Let $G_{ \pm \delta}=G\left(\phi_{ \pm \delta}\right)$ be saddle reductions of $G_{0}$ for a sufficiently small $\delta>0$ as in (b) of Figure 12 (i.e., $G_{-\delta}$ and $G_{+\delta}$ is related by a pop). Then, the Stokes phenomenon occurring to the Voros symbols are described explicitly the following jump formula.

Theorem 3.7 ([AIT] $)$. The Borel sums $\mathcal{S}_{ \pm}\left[e^{W_{\beta}}\right]$ and $\mathcal{S}_{ \pm}\left[e^{V_{\gamma}}\right]$ for any $\beta \in H_{1}\left(\hat{\Sigma} \backslash \hat{P}_{0}, \hat{P}_{\infty}\right)$ and any $\gamma \in H_{1}(\hat{\Sigma} \backslash \hat{P})$ satisfy the following equalities as analytic functions of $\eta$ on a domain containing $\{\eta \in \mathbb{R} \mid \eta \gg 1\}$ :

$$
\begin{aligned}
\mathcal{S}_{-}\left[e^{W_{\beta}}\right] & =\mathcal{S}_{+}\left[e^{W_{\beta}}\right]\left(1-\mathcal{S}_{+}\left[e^{V_{\gamma_{0}}}\right]\right)^{\left\langle\gamma_{0}, \beta\right\rangle}, \\
\mathcal{S}_{-}\left[e^{V_{\gamma}}\right] & =\mathcal{S}_{+}\left[e^{V_{\gamma}}\right] .
\end{aligned}
$$

The formula (3.13) is derived as a corollary of the main result of the forthcoming paper [AIT]. To make the paper self-contained, we will give an alternative proof of (3.13) in Appendix B. Note that the Borel sum of the Voros symbol $e^{V_{\gamma}}$ do not jump for any $\gamma \in H_{1}(\hat{\Sigma} \backslash \hat{P})$. This is a consequence of the first equality of (3.13) and the fact $\left(\gamma_{0}, \gamma\right)=0$ for any $\gamma \in H_{1}(\hat{\Sigma} \backslash \hat{P})$.

Moreover, we have

$$
\begin{aligned}
V_{\gamma_{0}}(\eta) & =\oint_{\gamma_{0}}\left(\eta \sqrt{Q_{0}(z)}+S_{\text {odd }}^{\mathrm{reg}}(z, \eta)\right) d z \\
& =\oint_{\gamma_{0}}\left(\eta \sqrt{Q_{0}(z)}\right) d z
\end{aligned}
$$

since $S_{\text {odd }}^{\text {reg }}(z, \eta) d z$ is holomorphic at the double pole $p$ by Proposition 2.8. This implies that the Voros symbol $e^{V_{\gamma_{0}}}$ for the saddle class $\gamma_{0}$ associated with a degenerate saddle trajectory is not a formal series but a scalar.

Similarly to (3.11), we also define a field automorphism $\mathfrak{K}_{\gamma_{0}}: \mathbb{V} \rightarrow \mathbb{V}$ by

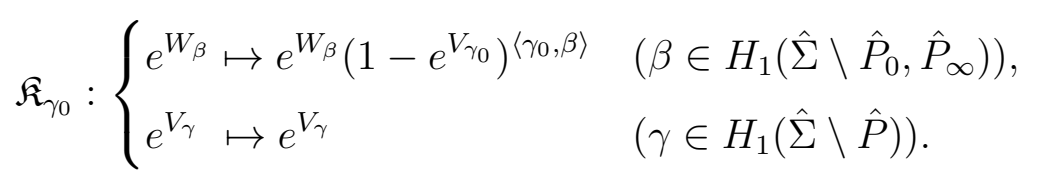

Then the map $\mathfrak{K}_{\gamma_{0}}$ satisfies

$$
\mathcal{S}_{-}=\mathcal{S}_{+} \circ \mathfrak{K}_{\gamma_{0}}
$$

The map $\mathfrak{K}_{\gamma_{0}}$ is called the Stokes automorphism for the saddle class $\gamma_{0}$ associated with a degenerate saddle trajectory $\ell_{0}$.

\section{6. $S^{1}$-action on potential and jump formulas}

Let us give an alternative interpretation of the jump formulas (3.10) and (3.13) in view of the deformation of the potential $Q(z, \eta)$. We consider a particular deformation realized by an action of the unit circle $S^{1}=\left\{e^{i \theta} \mid \theta \in \mathbb{R}\right\}$, which we call the $S^{1}$-action on the potential $Q(z, \eta)$. 
Suppose that the Stokes graph $G_{0}=G(\phi)$ has a unique regular or degenerate saddle trajectory $\ell_{0}$. Take a number $r>0$ and consider the family of Schrödinger equations

$$
\begin{aligned}
& \left(\frac{d^{2}}{d z^{2}}-\eta^{2} Q^{(\theta)}(z, \eta)\right) \psi^{(\theta)}(z, \eta)=0 \quad(-r \leq \theta \leq+r), \\
& Q^{(\theta)}(z, \eta)=Q_{0}^{(\theta)}(z)+\eta^{-1} Q_{1}^{(\theta)}(z)+\eta^{-2} Q_{2}^{(\theta)}(z)+\cdots .
\end{aligned}
$$

Here the family of potentials $\left\{Q^{(\theta)}(z, \eta) \mid-r \leq \theta \leq+r\right\}$ is defined by

$$
Q^{(\theta)}(z, \eta)=e^{2 i \theta} Q\left(z, e^{i \theta} \eta\right)
$$

where $Q(z, \eta)$ is the original potential of (2.1). We call this family the $S^{1}$-family for the potential $Q(z, \eta)$. Note that (3.18) satisfies Assumptions 2.3 and 2.5 for all $\theta \in[-r,+r]$. Taking $r>0$ sufficiently small, we may assume that the Stokes graph defined from $Q^{(\theta)}(z, \eta)$ is saddle-free if $\theta \neq 0, \theta \in[-r,+r]$. Since the principal terms of potentials satisfy

$$
Q_{0}^{(\theta)}(z)=e^{2 i \theta} Q_{0}(z)
$$

the quadratic differential associated with (3.17) is noting but $\phi_{\theta}$ defined in (2.39). The Stokes graph for $Q^{(0)}(z, \eta)=Q(z, \eta)$ coincides with the original Stokes graph $G_{0}$ containing the saddle trajectory $\ell_{0}$.

For any fixed $\theta$, let $S_{\text {odd }}^{(\theta)}(z, \eta)$ (resp., $S_{\text {odd }}^{\text {reg }(\theta)}(z, \eta)$ ) be the formal power series defined in the same manner as (2.14) (resp., (2.19)) from the Schrödinger equation (3.17). The following statement immediately follows from the uniqueness of formal solutions of the Riccati equation associated with (3.17) (see Section 2.3).

Lemma 3.8. The following identities holds:

$$
S_{\text {odd }}^{(\theta)}(z, \eta)=S_{\text {odd }}\left(z, e^{i \theta} \eta\right), \quad S_{\text {odd }}^{\mathrm{reg}(\theta)}(z, \eta)=S_{\text {odd }}^{\text {reg }}\left(z, e^{i \theta} \eta\right)
$$

We define the Voros symbols $e^{W_{\beta}^{(\theta)}}$ and $e^{V_{\gamma}^{(\theta)}}\left(\beta \in H_{1}\left(\hat{\Sigma} \backslash \hat{P}_{0} ; \hat{P}_{\infty}\right), \gamma \in H_{1}\left(\hat{\Sigma} \backslash \hat{P}_{0}\right)\right)$ of the Schrödinger equation (3.17) by

$$
W_{\beta}^{(\theta)}(\eta)=\int_{\beta} S_{\text {odd }}^{\mathrm{reg}(\theta)}(z, \eta) d z, \quad V_{\gamma}^{(\theta)}(\eta)=\oint_{\gamma} S_{\text {odd }}^{(\theta)}(z, \eta) d z .
$$

Note that, by (3.19), the Riemann surface $\hat{\Sigma}$ defined from (3.17) does not depend on $\theta$. Thus, the homology groups $H_{1}\left(\hat{\Sigma} \backslash \hat{P}_{0}\right)$ and $H_{1}\left(\hat{\Sigma} \backslash \hat{P}_{0} ; \hat{P}_{\infty}\right)$ for the Schrödinger equations (3.17) also do not depend on $\theta$. Thus, the equality (3.20) implies that

$$
W_{\beta}^{(\theta)}(\eta)=W_{\beta}\left(e^{i \theta} \eta\right), \quad V_{\gamma}^{(\theta)}(\eta)=V_{\gamma}\left(e^{i \theta} \eta\right)
$$

hold as formal series.

Lemma 3.9. For any $\theta \neq 0$ satisfying $-r \leq \theta \leq+r$, the formal series $e^{W_{\beta}^{(\theta)}}$ and $e^{V_{\gamma}^{(\theta)}}$ are Borel summable (in the direction 0 ), and the equalities

$$
\mathcal{S}\left[e^{W_{\beta}^{(\theta)}}\right](\eta)=\mathcal{S}_{\theta}\left[e^{W_{\beta}}\right]\left(e^{i \theta} \eta\right), \quad \mathcal{S}\left[e^{V_{\gamma}^{(\theta)}}\right](\eta)=\mathcal{S}_{\theta}\left[e^{V_{\gamma}}\right]\left(e^{i \theta} \eta\right)
$$

hold as analytic functions of $\eta$ on $\{\eta \in \mathbb{R} \mid \eta \gg 1\}$. 
Proof. Since the argument is the same, let us concentrate on the case of $W_{\beta}^{(\theta)}$. follows from (2.35) that the equality

$$
W_{\beta, B}^{(\theta)}(y)=e^{-i \theta} W_{\beta, B}\left(e^{-i \theta} y\right)
$$

holds near $y=0$. Here $W_{\beta, B}(y)$ and $W_{\beta, B}^{(\theta)}(y)$ are the Borel transform of $W_{\beta}(\eta)$ and $W_{\beta}^{(\theta)}(\eta)$, respectively. Since the quadratic differential $\phi_{\theta}$ is saddle-free, $W_{\beta}(\eta)$ is Borel summable in the direction $\theta$ by Corollary 2.21. Then, Lemma 2.12 implies that $W_{\beta}^{(\theta)}(\eta)$ is Borel summable in the direction 0 and we have the equality

$$
\mathcal{S}\left[W_{\beta}^{(\theta)}(\eta)\right]=\mathcal{S}_{\theta}\left[W_{\beta}\left(e^{i \theta} \eta\right)\right]
$$

by the definition (2.33) of the Borel sum in the direction $\theta$. Then the desired equality (3.23) follow from (3.24).

The equality (3.23) and Lemma 3.3 imply that the limit $\delta \rightarrow+0$ of the function $\mathcal{S}\left[e^{W_{\beta}^{( \pm \delta)}}\right](\eta)$ exists and coincides with $\mathcal{S}_{ \pm}\left[e^{W_{\beta}}\right](\eta)$ defined in Section 3.3. That is,

$$
\lim _{\delta \rightarrow+0} \mathcal{S}\left[e^{W_{\beta}^{( \pm \delta)}}\right](\eta)=\mathcal{S}_{ \pm}\left[e^{W_{\beta}}\right](\eta)
$$

holds on $\{\eta \in \mathbb{R} \mid \eta \gg 1\}$. Similarly, we also have

$$
\lim _{\delta \rightarrow+0} \mathcal{S}\left[e^{V_{\gamma}^{( \pm \delta)}}\right](\eta)=\mathcal{S}_{ \pm}\left[e^{V_{\gamma}}\right](\eta)
$$

Therefore, we obtain the following jump formulas for the $S^{1}$-action on the potential from Theorem 3.4 and 3.7 .

Theorem 3.10. (a). Suppose that $\ell_{0}$ is a regular saddle trajectory with the associated saddle class $\gamma_{0}$. Then we have

$$
\left\{\begin{array}{l}
\lim _{\delta \rightarrow+0} \mathcal{S}\left[e^{W_{\beta}^{(-\delta)}}\right](\eta)=\lim _{\delta \rightarrow+0}\left(\mathcal{S}\left[e^{W_{\beta}^{(+\delta)}}\right](\eta)\left(1+\mathcal{S}\left[e^{V_{\gamma_{0}}^{(+\delta)}}\right](\eta)\right)^{-\left\langle\gamma_{0}, \beta\right\rangle}\right), \\
\lim _{\delta \rightarrow+0} \mathcal{S}\left[e^{V_{\gamma}^{(-\delta)}}\right](\eta)=\lim _{\delta \rightarrow+0}\left(\mathcal{S}\left[e^{V_{\gamma}^{(+\delta)}}\right](\eta)\left(1+\mathcal{S}\left[e^{V_{\gamma_{0}}^{(+\delta)}}\right](\eta)\right)^{-\left(\gamma_{0}, \gamma\right)}\right),
\end{array}\right.
$$

for any $\beta \in H_{1}\left(\hat{\Sigma} \backslash \hat{P}_{0}, \hat{P}_{\infty}\right)$ and any $\gamma \in H_{1}(\hat{\Sigma} \backslash \hat{P})$.

(b). Suppose that $\ell_{0}$ is a degenerate saddle trajectory with the associated saddle class $\gamma_{0}$. Then we have

$$
\left\{\begin{array}{l}
\lim _{\delta \rightarrow+0} \mathcal{S}\left[e^{W_{\beta}^{(-\delta)}}\right](\eta)=\lim _{\delta \rightarrow+0}\left(\mathcal{S}\left[e^{W_{\beta}^{(+\delta)}}\right](\eta)\left(1-\mathcal{S}\left[e^{V_{\gamma_{0}}^{(+\delta)}}\right](\eta)\right)^{\left\langle\gamma_{0}, \beta\right\rangle}\right) \\
\lim _{\delta \rightarrow+0} \mathcal{S}\left[e^{V_{\gamma}^{(-\delta)}}\right](\eta)=\lim _{\delta \rightarrow+0} \mathcal{S}\left[e^{V_{\gamma}^{(+\delta)}}\right](\eta)
\end{array}\right.
$$

for any $\beta \in H_{1}\left(\hat{\Sigma} \backslash \hat{P}_{0}, \hat{P}_{\infty}\right)$ and any $\gamma \in H_{1}(\hat{\Sigma} \backslash \hat{P})$.

This concludes the exposition of the materials from the exact WKB analysis. We revisit the jump formulas and Stokes automorphisms in this section in view of cluster algebra theory later in Sections 7 and 8 . 


\section{Cluster algebras with coefficients}

In this section we summarize the basic notions and properties in cluster algebras which we will use in this paper. We also introduce the notion of signed mutations of seeds in Section 4.6 to accommodate the forthcoming results of this paper. We ask the readers to consult [FZ07, Nak12], for example, for further explanations if necessary, though it is our hope that the readers will not be bogged down in the cluster algebras machinery presented in this and the next sections, and smoothly proceed to Section 6 where our real work starts.

\subsection{Semifields}

Let us start from the notion of semifields, where "coefficients" of cluster algebras live.

Definition 4.1. A semifield $\mathbb{P}$ is a multiplicative abelian group endowed with an addition denoted by $\oplus$, which is commutative, associative, and distributive with respect to the multiplication.

To say it plainly, a semifield is almost a field, but without zero and subtraction. In this paper we mainly use the following examples.

Example 4.2. Let $u=\left(u_{i}\right)_{i=1}^{n}$ be an $n$-tuple of formal variables.

(a) The universal semifield $\mathbb{Q}_{+}(u)$ of $u$. This is the semifield of all nonzero rational functions of $u$ which have subtraction-free expressions, where the multiplication · and the addition $\oplus$ are defined by the usual $\times$ and + in the rational function field $\mathbb{Q}(u)$ of

$u$. As a standard example, the polynomial $u_{1}^{2}-u_{1} u_{2}+u_{2}^{2}$ does not seem to belong to $\mathbb{Q}_{+}(u)$; however, it actually does, since

$$
u_{1}^{2}-u_{1} u_{2}+u_{2}^{2}=\frac{u_{1}^{3}+u_{2}^{3}}{u_{1}+u_{2}}=\frac{u_{1}^{3} \oplus u_{2}^{3}}{u_{1} \oplus u_{2}} \in \mathbb{Q}_{+}(u) .
$$

(b) The tropical semifield $\operatorname{Trop}(u)$ of $u$. This is the multiplicative free abelian group generated by $u$, endowed with the tropical sum $\oplus$ defined by

$$
\prod_{i=1}^{n} u_{i}^{a_{i}} \oplus \prod_{i=1}^{n} u_{i}^{b_{i}}:=\prod_{i=1}^{n} u_{i}^{\min \left(a_{i}, b_{i}\right)} .
$$

It is called so because it is essentially the exponential and multivariable version of the tropical semiring (also known as the min-plus algebra) $a \oplus b:=\min (a, b), a \otimes b:=a+b$. The tropical semiring is the central object of the tropical mathematics well studied since 90's. See [SS09] for the subject and the explanation for this peculiar terminology.

(c) The tropicalization map. There is the natural semifield homomorphism

$$
\begin{aligned}
\pi_{\text {trop }}: \mathbb{Q}_{+}(u) & \rightarrow \operatorname{Trop}(u) \\
u_{i} & \mapsto u_{i} \\
c & \mapsto \quad 1 \quad\left(c \in \mathbb{Q}_{+}\right) .
\end{aligned}
$$


For example,

$$
\pi_{\text {trop }}: \frac{2 u_{1}^{2} \oplus u_{1} u_{2}}{u_{1}^{2} u_{2} \oplus 2 u_{1} u_{2}^{2}}=\frac{u_{1}\left(2 u_{1} \oplus u_{2}\right)}{u_{1} u_{2}\left(u_{1} \oplus 2 u_{2}\right)} \mapsto \frac{u_{1}}{u_{1} u_{2}}=u_{2}^{-1},
$$

where we used (4.2). So, plainly speaking, the tropicalization map $\pi_{\text {trop }}$ is the operation of taking the "principal term" (in the sense of (4.4)) of a rational function in $\mathbb{Q}_{+}(u)$.

For a given semifield $\mathbb{P}$, let $\mathbb{Z P}$ denote the group ring of $\mathbb{P}$ over $\mathbb{Z}$. Namely, $\mathbb{Z} \mathbb{P}$ is the commutative ring of all formal finite sums $\sum_{r=1}^{m} n_{r} p_{r}\left(n_{r} \in \mathbb{Z}, p_{r} \in \mathbb{P}\right)$. Note that we have two kinds of additions, $\oplus$ for $\mathbb{P}$ and the addition + for the group ring. For $\oplus$, there is no subtraction, but for + , there is the usual subtraction - in $\mathbb{Z} \mathbb{P}$. It is known that $\mathbb{Z P}$ is a domain [FZ02, Section 1.2]; namely, it has no zero divisors. Thus, the field of fractions $\mathbb{Q P}$ of the ring $\mathbb{Z P}$ is well-defined.

\subsection{Mutation of seeds and cluster algebra with coefficients}

Let us recall the notions of mutations of seeds and cluster algebras, following [FZ03, FZ07].

To introduce a cluster algebra (with coefficients), let us first fix a positive integer $n$ called the rank, and a semifield $\mathbb{P}$ called the coefficient semifield. We choose an $n$-tuple of formal variables, say, $w=\left(w_{1}, \ldots, w_{n}\right)$, and consider the field of the rational functions in $w$ with coefficients in $\mathbb{Q P}$, which denoted by $\mathbb{Q P}(w)$.

A (labeled) seed $(B, x, y)$ with coefficients in $\mathbb{P}$ is a triplet with the following data:

- an exchange matrix $B=\left(b_{i j}\right)_{i, j=1}^{n}$, which is a skew-symmetric integer matrix,

- a cluster $x=\left(x_{i}\right)_{i=1}^{n}$, which is an $n$-tuple of algebraically independent elements in $\mathbb{Q P}(w)$ over $\mathbb{Q P}$,

- a coefficient tuple $y=\left(y_{i}\right)_{i=1}^{n}$, which is an $n$-tuple of elements in $\mathbb{P}$.

Each $x_{i}$ and $y_{i}$ are called a cluster variable and coefficient, respectively. In this paper we call them, a little casually, an $x$-variable and a $y$-variable, respectively. (They correspond to an $\mathcal{A}$-coordinate and an $\mathcal{X}$-coordinate in [FG09a], respectively.)

For any seed $(B, x, y)$ and any $k=1, \ldots, n$, we define another seed $\left(B^{\prime}, x^{\prime}, y^{\prime}\right)$, called the mutation of $(B, x, y)$ at $k$ and denoted by $\mu_{k}(B, x, y)$, by the following relations:

$$
\begin{aligned}
& b_{i j}^{\prime}= \begin{cases}-b_{i j} & i=k \text { or } j=k \\
b_{i j}+\left[-b_{i k}\right]_{+} b_{k j}+b_{i k}\left[b_{k j}\right]_{+} & i, j \neq k,\end{cases} \\
& y_{i}^{\prime}= \begin{cases}y_{k}{ }^{-1} & i=k \\
y_{i} \frac{\left(1 \oplus y_{k}\right)^{\left[-b_{k i}\right]_{+}}}{\left(1 \oplus y_{k}{ }^{-1}\right)^{\left[b_{k i}\right]_{+}}} & i \neq k,\end{cases} \\
& x_{i}^{\prime}= \begin{cases}x_{k}{ }^{-1}\left(\frac{1}{1 \oplus y_{k}^{-1}} \prod_{j=1}^{n} x_{j}{ }^{\left[b_{j k}\right]_{+}}+\frac{1}{1 \oplus y_{k}} \prod_{j=1}^{n} x_{j}^{\left[-b_{j k}\right]_{+}}\right) & i=k \\
x_{i} & i \neq k .\end{cases}
\end{aligned}
$$


Here, for any integer $a$, we set $[a]_{+}:=\max (a, 0)$. The above relations are called the exchange relations. The involution property $\mu_{k}^{2}=\mathrm{id}$ holds.

Definition 4.3. Let us fix an arbitrary seed $\left(B^{0}, x^{0}, y^{0}\right)$ with coefficients in $\mathbb{P}$, and call it the initial seed. Then, repeat mutations from the initial seed to all directions. Let $\operatorname{Seed}\left(B^{0}, x^{0}, y^{0} ; \mathbb{P}\right)$ denote the set of all so obtained seeds, including the initial one; namely,

$$
\operatorname{Seed}\left(B^{0}, x^{0}, y^{0} ; \mathbb{P}\right)=\left\{\mu_{k_{N}} \cdots \mu_{k_{1}}\left(B^{0}, x^{0}, y^{0}\right) \mid N \geq 0 ; k_{1}, \ldots, k_{N} \in\{1, \ldots, n\}\right\} .
$$

The cluster algebra $\mathcal{A}\left(B^{0}, x^{0}, y^{0} ; \mathbb{P}\right)$ with coefficients in $\mathbb{P}$ is the $\mathbb{Z} \mathbb{P}$-subalgebra of $\mathbb{Q P}(w)$ generated by all $x$-variables belonging to seeds in $\operatorname{Seed}\left(B^{0}, x^{0}, y^{0} ; \mathbb{P}\right)$. A seed in $\operatorname{Seed}\left(B^{0}, x^{0}, y^{0} ; \mathbb{P}\right)$ is called a seed of $\mathcal{A}\left(B^{0}, x^{0}, y^{0} ; \mathbb{P}\right)$.

What is important in our application is not the algebra $\mathcal{A}\left(B^{0}, x^{0}, y^{0} ; \mathbb{P}\right)$ itself but the exchange relations (4.5)-(4.7). They are the abstraction of relations occurring in Lie theory due to Fomin and Zelevinsky. For example, the relation (4.7) typically appears as relations in the coordinate rings of certain algebraic varieties related to Lie group, e.g., Grassmannians, $S L(n)$, etc. The relation (4.6) is a sort of the "dual" of the relation (4.7) as we see below. As explained at the beginning of Section 1, it is nowadays known that the cluster algebra structure, (i.e., the exchange relations (4.5)(4.7) ) serves a common underlying algebraic/combinatorial structure in several branches of mathematics - from algebra, geometry, analysis to combinatorics. Such ubiquity as a common structure reminds us of root systems. Indeed, cluster algebra theory may be regarded as an extended theory of root systems in several aspects. An explicit example of the exchange relations (4.5)-(4.7) will be exhibited in Example 4.10 later.

For each seed $(B, x, y)$ with coefficients in $\mathbb{P}$, we define $\hat{y}$-variables $\hat{y}_{1}, \ldots, \hat{y}_{n} \in$ $\mathbb{Q P}(w)$ by

$$
\hat{y}_{i}=y_{i} \prod_{j=1}^{n} x_{j}^{b_{j i}}
$$

It is easy to verify the following property by using (4.5)-(4.7).

Proposition 4.4 ([FZ07, Prop. 3.9]). Under the mutation $\left(B^{\prime}, x^{\prime}, y^{\prime}\right)=\mu_{k}(B, x, y)$ the following relation holds:

$$
\hat{y}_{i}^{\prime}= \begin{cases}\hat{y}_{k}{ }^{-1} & i=k \\ \hat{y}_{i} \frac{\left(1+\hat{y}_{k}\right)^{\left[-b_{k i}\right]_{+}}}{\left(1+\hat{y}_{k}{ }^{-1}\right)^{\left[b_{k i}\right]_{+}}} & i \neq k .\end{cases}
$$

In other words, $\hat{y}$-variables mutate just in the same way as $y$-variables.

\subsection{Quivers}

It is often convenient to represent a skew-symmetric matrix $B=\left(b_{i j}\right)_{i, j=1}^{n}$ by a (labeled) quiver $Q$ whose vertices are labeled by $1, \ldots, n$. In our convention, we write $b_{i j}$ arrows 
from vertex $i$ to vertex $j$ if and only if $b_{i j}>0$. This gives a one-to-one correspondence between skew-symmetric matrices and quivers without any loops (1-cycles) and oriented 2-cycles. Here is an example:

$$
B=\left(\begin{array}{ccc}
0 & -1 & 2 \\
1 & 0 & -1 \\
-2 & 1 & 0
\end{array}\right) \longleftrightarrow Q=\quad \stackrel{1}{3}
$$

In terms of quivers, the exchange relation (4.5) for the mutation at $k$ is translated as follows.

Step 1. For each pair of an arrow from $i$ to $k$ and an arrow from $k$ to $j$, add an arrow from $i$ to $j$.

Step 2. Reverse all arrows incident with $k$.

Step 3. Remove the arrows in a maximal set of pairwise disjoint 2-cycles.

For example, for the quiver $Q$ in (4.11), the mutation at 1 is done in the following manner.

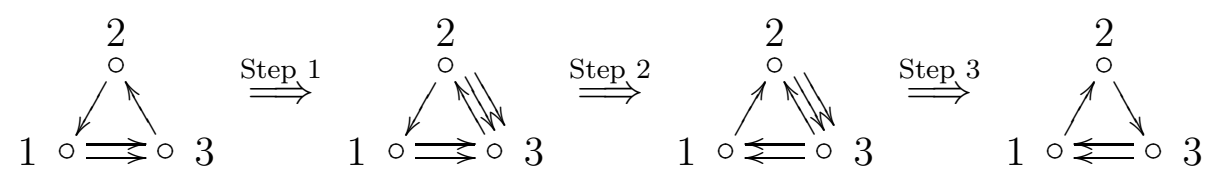

\subsection{Tropicalization of y-variables and tropical sign}

In this paper we mainly use the following two choices of $y$-variables. (See Example 4.2.)

(a). We set the coefficient semifield as $\mathbb{Q}_{+}\left(y^{0}\right)$ with $y^{0}=\left(y_{1}^{0}, \ldots, y_{n}^{0}\right)$; furthermore, we set the initial $y$-variables as $y^{0}$. We call the $y$-variables of $\mathcal{A}\left(B^{0}, x^{0}, y^{0} ; \mathbb{Q}_{+}\left(y^{0}\right)\right)$ the universal y-variables.

(b). We set the coefficient semifield as $\operatorname{Trop}\left(y^{0}\right)$ with $y^{0}=\left(y_{1}^{0}, \ldots, y_{n}^{0}\right)$; furthermore, we set the initial $y$-variables as $y^{0}$. We call the $y$-variables of $\mathcal{A}\left(B^{0}, x^{0}, y^{0} ; \operatorname{Trop}\left(y^{0}\right)\right)$ the tropical $y$-variables. (It is more standard to call them the principal coefficients [FZ07], but here we emphasize their tropical nature.)

The tropical $y$-variables are obtained from the universal $y$-variables by applying the tropicalization map in Example 4.2,

$$
\pi_{\text {trop }}: \mathbb{Q}_{+}\left(y^{0}\right) \rightarrow \operatorname{Trop}\left(y^{0}\right) .
$$

Namely, for any universal $y$-variable $y_{i} \in \mathbb{Q}_{+}\left(y^{0}\right)$, let $\left[y_{i}\right]:=\pi_{\text {trop }}\left(y_{i}\right) \in \operatorname{Trop}\left(y^{0}\right)$. Since $\pi_{\text {trop }}$ is a semifield homomorphism, it preserves the exchange relation (4.6); therefore, it commutes with mutations. Thus, $\left[y_{i}\right]$ is a tropical $y$-variable. From now on we conveniently use this expression for tropical $y$-variables.

By definition, a tropical $y$-variable $\left[y_{i}\right]$ is a Laurent monomial of the initial tropical $y$-variables $y^{0}$; namely, it is written in the form

$$
\left[y_{i}\right]=\prod_{j=1}^{n}\left(y_{j}^{0}\right)^{c_{j}},
$$


where $c=c\left(y_{i}\right)=\left(c_{j}\right)_{j=1}^{n}$ is an integer vector depending on $y_{i}$. The vector $c\left(y_{i}\right)$ is introduced in [FZ07] and called the c-vector of $y_{i}$.

We say that an integer vector is positive (resp., negative) if it is a nonzero vector and its components are all nonnegative (resp., nonpositive). We have the following important property of $c$-vectors.

Theorem 4.5 (Sign coherence of $c$-vectors ([FZ07, Prop. 5.7], [DWZ10, Theorem 1.7])). Any c-vector is either a positive vector or a negative vector.

Thanks to the theorem, we have the notion of the tropical sign.

Definition 4.6. Let $\mathcal{A}\left(B^{0}, x^{0}, y^{0} ; \mathbb{Q}_{+}\left(y^{0}\right)\right)$ be a cluster algebra with universal $y$ variables, and let $(B, x, y)$ be its seed. Then, to each component $y_{i}$ of $y$ we assign the tropical sign $\varepsilon\left(y_{i}\right)$ as $\varepsilon\left(y_{i}\right)=+$ (resp., $\varepsilon\left(y_{i}\right)=-$ ) if the $c$-vector $c\left(y_{i}\right)$ is positive (resp., negative).

Throughout the paper we conveniently identify the signs $\varepsilon= \pm$ and the numbers $\varepsilon= \pm 1$.

It is important that the tropical sign is a relative concept depending on $(B, x, y)$ and the initial seed $\left(B^{0}, x^{0}, y^{0}\right)$. By the definition of the tropical sign, we have

$$
1 \oplus\left[y_{i}\right]^{\varepsilon\left(y_{i}\right)}=1
$$

in $\operatorname{Trop}\left(y^{0}\right)$.

\subsection{E-expression of exchange relations}

Let us focus on some fine property of the exchange relations (4.6) and (4.7). It is easy to check that (4.6) and (4.7) can be expressed alternatively as [Kel11, Nak12.

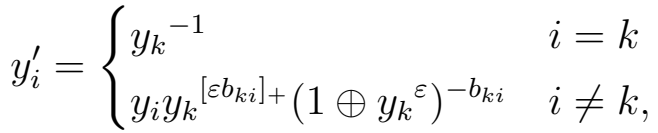

$$
\begin{aligned}
& x_{i}^{\prime}= \begin{cases}x_{k}{ }^{-1}\left(\prod_{j=1}^{n} x_{j}^{\left[-\varepsilon b_{j k}\right]_{+}}\right) \frac{1+\hat{y}_{k}^{\varepsilon}}{1 \oplus y_{k}^{\varepsilon}} & i=k \\
x_{i} & i \neq k,\end{cases}
\end{aligned}
$$

where $\varepsilon \in\{+,-\}=\{1,-1\}$, and the right hand sides are independent of the choice of $\varepsilon$. We call them the $\varepsilon$-expression of the exchange relations.

Let us specialize the $y$-variables $y_{i}$ in (4.16) and (4.17) to tropical $y$-variables [ $\left.y_{i}\right]$; furthermore, let us specialize $\varepsilon$ therein to the tropical sign $\varepsilon\left(y_{k}\right)$. Then, by (4.15), the 
relations (4.16) and (4.17) reduce to the following ones:

$$
\begin{aligned}
{\left[y_{i}^{\prime}\right] } & = \begin{cases}{\left[y_{k}\right]^{-1}} & i=k \\
{\left[y_{i}\right]\left[y_{k}\right]^{\left[\varepsilon\left(y_{k}\right) b_{k i}\right]_{+}}} & i \neq k,\end{cases} \\
x_{i}^{\prime} & = \begin{cases}x_{k}{ }^{-1}\left(\prod_{j=1}^{n} x_{j}^{\left[-\varepsilon\left(y_{k}\right) b_{j k}\right]_{+}}\right)\left(1+\hat{y}_{k}^{\varepsilon\left(y_{k}\right)}\right) & i=k \\
x_{i} & i \neq k,\end{cases}
\end{aligned}
$$

where $\hat{y}_{k}=\left[y_{k}\right] \prod_{j=1}^{n} x_{j}^{b_{j i}}$. These are an alternative expressions of the exchange relations for tropical $y$-variables and $x$-variables with tropical $y$-variables as coefficients.

\subsection{Signed mutations}

We introduce some new notions in cluster algebras, motivated by the forthcoming results in this paper.

For any seed $(B, x, y)$ with coefficients in $\operatorname{Trop}\left(y^{0}\right)$, any $k=1, \ldots, n$, and any sign $\varepsilon \in\{+,-\}$, we introduce the signed monomial mutation $\left(B^{\prime}, x^{\prime}, y^{\prime}\right)=m_{k}^{(\varepsilon)}(B, x, y)$ by the following exchange relation, where $B^{\prime}$ is defined as usual:

$$
\begin{aligned}
& y_{i}^{\prime}= \begin{cases}y_{k}^{-1} & i=k \\
y_{i} y_{k}{ }^{\left[\varepsilon b_{k i}\right]_{+}} & i \neq k,\end{cases} \\
& x_{i}^{\prime}= \begin{cases}x_{k}{ }^{-1} \prod_{j=1}^{n} x_{j}{ }^{\left[-\varepsilon b_{j k}\right]_{+}} & i=k \\
x_{i} & i \neq k .\end{cases}
\end{aligned}
$$

Unlike (4.18) and (4.19), they depend on $\varepsilon$. If we set $\varepsilon$ to the tropical sign $\varepsilon\left(y_{k}\right)$ for the universal $y$-variables, the relation (4.20) reduces to the exchange relation (4.18) of the tropical $y$-variables. Starting from a given initial seed $\left(B^{0}, x^{0}, y^{0}\right)$, we obtain a family of seeds by repeating the above mutations to any direction $k$ and any $\operatorname{sign} \varepsilon$. We call so obtained $x_{i}$ 's and $y_{i}$ 's the monomial $x$-variables and the monomial $y$-variables, respectively.

In the same setting of seeds, we also consider another kind of mutation, the signed mutation $\left(B^{\prime}, x^{\prime}, y^{\prime}\right)=\mu_{k}^{(\varepsilon)}(B, x, y)$, by keeping (4.20) and replacing (4.21) by the following:

$$
x_{i}^{\prime}= \begin{cases}x_{k}{ }^{-1}\left(\prod_{j=1}^{n} x_{j}{ }^{\left[-\varepsilon b_{j k}\right]_{+}}\right)\left(1+\hat{y}_{k}{ }^{\varepsilon}\right) & i=k \\ x_{i} & i \neq k\end{cases}
$$

where $\hat{y}_{k}=y_{k} \prod_{j=1}^{n} x_{j}^{b_{j k}}$. Again, it depends on $\varepsilon$. If we set $\varepsilon$ to the tropical sign $\varepsilon\left(y_{k}\right)$ of the universal $y$-variables, then the relation (4.22) reduces to the exchange relation (4.19) of $x$-variables with tropical $y$-variables as coefficients.

We have a natural extension of Proposition 4.4. 
Proposition 4.7. Under the signed mutation $\left(B^{\prime}, x^{\prime}, y^{\prime}\right)=\mu_{k}^{(\varepsilon)}(B, x, y)$ with the exchange relations (4.20) and (4.22), $\hat{y}$-variables $\hat{y}_{i}=y_{i} \prod_{j=1}^{n} x_{j}^{b_{j i}}$ satisfy the exchange relation

$$
\hat{y}_{i}^{\prime}= \begin{cases}\hat{y}_{k}{ }^{-1} & i=k \\ \hat{y}_{i} \hat{y}_{k}{ }^{\left[\varepsilon b_{k i}\right]_{+}}\left(1+\hat{y}_{k}{ }^{\varepsilon}\right)^{-b_{k i}} & i \neq k,\end{cases}
$$

which is equivalent to (4.10). In particular, the mutation of $\hat{y}$-variables does not depend on the sign $\varepsilon$.

The proof can be done in a similar (and a little easier) calculation as for Proposition 4.4. However, the result is new in the literature.

\subsection{Periodicity in cluster algebras}

Let us introduce the notion of periodicity in a cluster algebra. We call a sequence $\vec{k}=\left(k_{t}\right)_{t=1}^{N}$ with $k_{t} \in\{1, \ldots, n\}$ a mutation sequence, and we naturally identify it with the sequence (composition) of mutations $\mu_{\vec{k}}:=\mu_{k_{N}} \circ \cdots \circ \mu_{1}$.

Theorem 4.8. Let $\mathcal{A}\left(B^{0}, x^{0}, y^{0} ; \mathbb{Q}_{+}\left(y^{0}\right)\right)$ be a cluster algebra with universal y-variables, and let $\vec{k}=\left(k_{t}\right)_{t=1}^{N}$ be a mutation sequence. Let $(B, x, y)$ and $\left(B^{\prime}, x^{\prime}, y^{\prime}\right)$ be seeds of $\mathcal{A}\left(B^{0}, x^{0}, y^{0} ; \mathbb{Q}_{+}\left(y^{0}\right)\right)$ such that $\left(B^{\prime}, x^{\prime}, u^{\prime}\right)=\mu_{\vec{k}}(B, x, y)$, and let $\nu$ be a permutation of $\{1, \ldots, n\}$. Then, the following conditions are equivalent.

(a). $b_{\nu(i) \nu(j)}^{\prime}=b_{i j}, x_{\nu(i)}^{\prime}=x_{i}$, and $y_{\nu(i)}^{\prime}=y_{i}$ hold for any $i$ and $j$.

(b). $y_{\nu(i)}^{\prime}=y_{i}$ holds for any $i$.

(c). $x_{\nu(i)}^{\prime}=x_{i}$ holds for any $i$.

(d). $\left[y_{\nu(i)}^{\prime}\right]=\left[y_{i}\right]$ holds for any $i$.

(e). $\left[x_{\nu(i)}^{\prime}\right]=\left[x_{i}\right]$ holds for any $i$, where $\left[x_{i}\right] \in \mathbb{Q}\left(\operatorname{Trop}\left(y^{0}\right)\right)(w)$ is the one obtained from $x_{i}$ by the tropicalization of $y$-variables.

Proof. The implications (a) $\Rightarrow(\mathrm{b}) \Rightarrow(\mathrm{d})$ and $(\mathrm{a}) \Rightarrow(\mathrm{c}) \Rightarrow(\mathrm{e})$ are obvious, while (d) $\Rightarrow(\mathrm{a})$ is the result of $\mathrm{IIK}^{+} 13 \mathrm{a}$, Pla11. Let us show $(e) \Rightarrow(a)$. It follows from the assumption (e) that the corresponding $g$-vectors in [FZ07] have the same periodicity. Then, the claim (a) follows again from the result of [Pla11].

Definition 4.9. A mutation sequence $\vec{k}=\left(k_{t}\right)_{t=1}^{N}$ is called a $\nu$-period of $(B, x, y)$ if one of the conditions (a)-(e) in Theorem 4.8 holds for the seed $\left(B^{\prime}, x^{\prime}, y^{\prime}\right)=\mu_{\vec{k}}(B, x, y)$.

Many interesting examples of periodicities of seeds are known [FZ07, Kel10, $\mathrm{IIK}^{+} 13 \mathrm{a}$, IIK ${ }^{+} 13 \mathrm{~b}$, NS12]. Here, we give the simplest example, which will be used as the running example throughout the paper.

Example 4.10 (Pentagon relation (1)). Consider the cluster algebra $\mathcal{A}\left(B^{0}, x^{0}, y^{0} ; \mathbb{Q}_{+}\left(y^{0}\right)\right.$ ) whose initial exchange matrix $B^{0}$ and the corresponding quiver $Q^{0}$ are given by

$$
B^{0}=\left(\begin{array}{cc}
0 & 1 \\
-1 & 0
\end{array}\right), \quad Q^{0}=\underset{1}{\stackrel{\circ}{\longrightarrow}} \underset{2}{\longrightarrow} .
$$


This is the cluster algebra of type $A_{2}$ in the classification of [FZ02]. In particular, it is of finite type, namely, there are only finitely many seeds. Set $(B(1), x(1), y(1))$ to be the initial seed $\left(B^{0}, x^{0}, y^{0}\right)$, and consider the mutation sequence $\vec{k}=(1,2,1,2,1)$, i.e.,

$$
(B(1), x(1), y(1)) \stackrel{\mu_{1}}{\rightarrow}(B(2), x(2), y(2)) \stackrel{\mu_{2}}{\rightarrow} \cdots \stackrel{\mu_{1}}{\rightarrow}(B(6), x(6), y(6)) .
$$

For simplicity, we write the initial variables $x_{i}=x_{i}^{0}$ and $y_{i}=y_{i}^{0}$. According to (4.9), we set the initial $\hat{y}$-variables as

$$
\hat{y}_{1}=y_{1} x_{2}^{-1}, \quad \hat{y}_{2}=y_{2} x_{1} .
$$

Then, using the exchange relations (4.6) and (4.17), we obtain the following explicit form of seeds:

$$
\begin{aligned}
& Q(1) \underset{1}{\longrightarrow} \underset{2}{\longrightarrow} \underset{2}{\bigcirc}\left\{\begin{array} { l } 
{ x _ { 1 } ( 1 ) = x _ { 1 } } \\
{ x _ { 2 } ( 1 ) = x _ { 2 } , }
\end{array} \quad \left\{\begin{array}{l}
y_{1}(1)=y_{1} \\
y_{2}(1)=y_{2},
\end{array}\right.\right. \\
& Q(2) \underset{1}{\circ}\left\{\begin{array} { l } 
{ x _ { 1 } ( 2 ) = x _ { 1 } ^ { - 1 } x _ { 2 } \frac { 1 + \hat { y } _ { 1 } } { 1 \oplus y _ { 1 } } } \\
{ x _ { 2 } ( 2 ) = x _ { 2 } , }
\end{array} \quad \left\{\begin{array}{l}
y_{1}(2)=y_{1}^{-1} \\
y_{2}(2)=y_{1} y_{2}\left(1 \oplus y_{1}\right)^{-1}
\end{array}\right.\right. \\
& Q(3) \underset{1}{\longrightarrow} \underset{2}{\longrightarrow} \underset{2}{\longrightarrow}\left\{\begin{array} { l } 
{ x _ { 1 } ( 3 ) = x _ { 1 } ^ { - 1 } x _ { 2 } \frac { 1 + \hat { y } _ { 1 } } { 1 \oplus y _ { 1 } } } \\
{ x _ { 2 } ( 3 ) = x _ { 1 } ^ { - 1 } \frac { 1 + \hat { y } _ { 1 } + \hat { y } _ { 1 } \hat { y } _ { 2 } } { 1 \oplus y _ { 1 } \oplus y _ { 1 } y _ { 2 } } , }
\end{array} \quad \left\{\begin{array}{l}
y_{1}(3)=y_{2}\left(1 \oplus y_{1} \oplus y_{1} y_{2}\right)^{-1} \\
y_{2}(3)=y_{1}^{-1} y_{2}^{-1}\left(1 \oplus y_{1}\right),
\end{array}\right.\right. \\
& Q(4) \underset{1}{2}\left\{\begin{array} { l } 
{ x _ { 1 } ( 4 ) = x _ { 2 } ^ { - 1 } \frac { 1 + \hat { y } _ { 2 } } { 1 \oplus y _ { 2 } } } \\
{ x _ { 2 } ( 4 ) = x _ { 1 } ^ { - 1 } \frac { 1 + \hat { y } _ { 1 } + \hat { y } _ { 1 } \hat { y } _ { 2 } } { 1 \oplus y _ { 1 } \oplus y _ { 1 } y _ { 2 } } , }
\end{array} \quad \left\{\begin{array}{l}
y_{1}(4)=y_{2}^{-1}\left(1 \oplus y_{1} \oplus y_{1} y_{2}\right) \\
y_{2}(4)=y_{1}^{-1}\left(1 \oplus y_{2}\right)^{-1},
\end{array}\right.\right. \\
& Q(5) \underset{1}{\stackrel{0}{\longrightarrow}} \underset{2}{\longrightarrow}\left\{\begin{array} { l } 
{ x _ { 1 } ( 5 ) = x _ { 2 } ^ { - 1 } \frac { 1 + \hat { y } _ { 2 } } { 1 \oplus y _ { 2 } } } \\
{ x _ { 2 } ( 5 ) = x _ { 1 } , }
\end{array} \quad \left\{\begin{array}{l}
y_{1}(5)=y_{2}^{-1} \\
y_{2}(5)=y_{1}\left(1 \oplus y_{2}\right),
\end{array}\right.\right. \\
& Q(6) \underset{1}{\mathrm{O}} \mathrm{\rho}\left\{\begin{array} { l } 
{ x _ { 1 } ( 6 ) = x _ { 2 } } \\
{ x _ { 2 } ( 6 ) = x _ { 1 } . }
\end{array} \quad \left\{\begin{array}{l}
y_{1}(6)=y_{2} \\
y_{2}(6)=y_{1}
\end{array}\right.\right.
\end{aligned}
$$

Here, the encircled vertices in quivers are the mutation points in the sequence (4.25). We see that the mutation sequence $\vec{k}$ is a $\nu$-period of $(B(1), x(1), y(1))$, where $\nu=(12)$ is the permutation of 1 and 2 . This periodicity is known as the pentagon relation. The tropical $y$-variables at the mutation points are

$$
\left[y_{1}(1)\right]=y_{1}, \quad\left[y_{2}(2)\right]=y_{1} y_{2}, \quad\left[y_{1}(3)\right]=y_{2}, \quad\left[y_{2}(4)\right]=y_{1}^{-1}, \quad\left[y_{1}(5)\right]=y_{2}^{-1},
$$

and the corresponding tropical signs $\varepsilon_{t}=\varepsilon\left(y_{k_{t}}(t)\right)$ are

$$
\varepsilon_{1}=+, \quad \varepsilon_{2}=+, \quad \varepsilon_{3}=+, \quad \varepsilon_{4}=-, \quad \varepsilon_{5}=-.
$$




\section{Surface realization of cluster algebras}

There is a class of cluster algebras which can be realized (in various sense) by triangulations of surfaces GSV05, FG06, FST08, FT12. This construction is often referred to as the surface realization of cluster algebras. Since careful treatment of mutations involving a self-folded triangle is crucial throughout the paper, we explain in detail how there are related to tagged triangulations and signed triangulations. We mostly follow [FST08, FT12, but we do some reformulation to work with labeled triangulations. The extended seeds and their signed mutations and pops are also defined.

\subsection{Ideal triangulations of bordered surface with marked points}

To start, we choose a compact connected oriented surface possibly with boundary $\mathbf{S}$, and a finite set $\mathbf{M}$ of marked points on $\mathbf{S}$ that is nonempty and includes at least one marked point on each boundary component and possibly some interior points. If a marked point is an interior point of $\mathbf{S}$, then it is called a puncture. We impose the following assumption by a technical reason [FST08]:

Assumption 5.1. The following cases of $(\mathbf{S}, \mathbf{M})$ are excluded:

- a sphere with less than four punctures,

- an unpunctured or once-punctured monogon,

- an unpunctured digon,

- an unpunctured triangle.

In the above a surface homeomorphic to a disk with $n$ marked points on the boundary is called a polygon, and, in particular, monogon, digon, triangle for $n=1,2,3$, respectively.

A pair ( $\mathbf{S}, \mathbf{M})$ satisfying Assumption 5.1 is called a bordered surface with marked points, or a bordered surface, for simplicity.

Remark 5.2. In [FST08, FT12] $\mathbf{S}$ is assumed to be a Riemann surface. In our application we need neither a complex structure nor a metric.

First we consider triangulations of $(\mathbf{S}, \mathbf{M})$ by ordinary arcs, where "ordinary" means "not tagged" which will be introduced later.

Definition 5.3. An arc $\alpha$ in a bordered surface $(\mathbf{S}, \mathbf{M})$ is a curve in $\mathbf{S}$ such that

- the endpoints of $\alpha$ are marked points,

- $\alpha$ does not intersect itself except for the endpoints,

- $\alpha$ is away from punctures and boundaries except for the endpoints,

- $\alpha$ is not contractible into a marked point or onto a boundary of $\mathbf{S}$.

Furthermore, each $\operatorname{arc} \alpha$ is considered up to isotopy in the class of such curves. For example, "distinct (resp. identical) arcs" means distinct (resp. identical) isotopy classes of curves unless otherwise mentioned. 


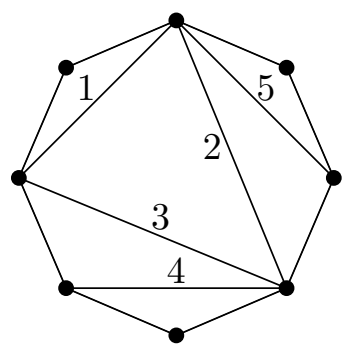

(a)

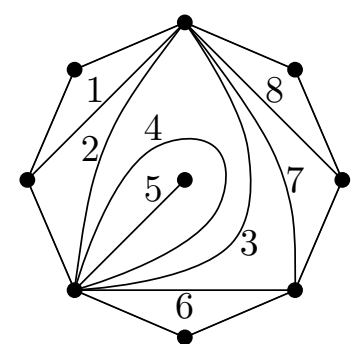

(b)

Figure 14. Examples of labeled ideal triangulations of a polygon without puncture (a) and a polygon with one puncture (b).

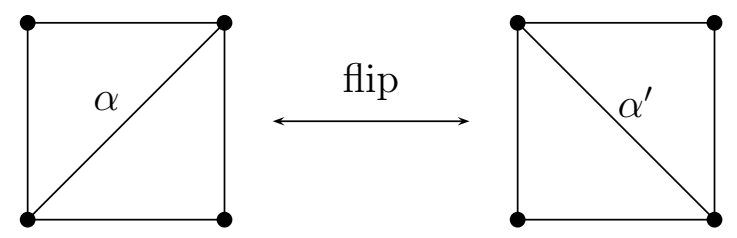

Figure 15. Flip of a diagonal arc.

Two arcs are said to be compatible if there are representatives in their respective isotopy classes such that they do not intersect each other in the interior of $\mathbf{S}$.

Definition 5.4. An ideal triangulation $T=\left\{\alpha_{i}\right\}_{i \in I}$ of $(\mathbf{S}, \mathbf{M})$ is a maximal set of distinct pairwise compatible arcs in $(\mathbf{S}, \mathbf{M})$.

See Figure 14 for examples of ideal triangulations. We also put labels $1,2, \ldots$ to the arcs for the later use. As in the second example, some degenerate triangles, such as selffolded triangles (e.g., 4-5) and triangles with identified vertices (e.g., 2-3-4), may appear around a puncture. From now on we also call them triangles (of an ideal triangulation).

Definition 5.5. For an ideal triangulation $T$ of $(\mathbf{S}, \mathbf{M})$ and an $\operatorname{arc} \alpha \in T$, if there is another arc $\alpha^{\prime}$ such that that $T^{\prime}=(T-\{\alpha\}) \cup\left\{\alpha^{\prime}\right\}$ is an ideal triangulation, then $\alpha^{\prime}$ is called a flip of $\alpha$, and $T^{\prime}$ is called a flip of $T$ at $\alpha$. (As wee see soon, such $\alpha^{\prime}$ is unique for each $\alpha$ if it exists.)

Now we encounter a problem that not all arcs are flippable. For example, in the triangulation in Figure 14 (b), the arc with label 5 is not flippable. Luckily this is the only situation where an arc is not flippable. Indeed, if an $\operatorname{arc} \alpha$ is the inner side of a selffolded triangle (an inner arc, for short), it is not flippable. Otherwise, $\alpha$ is the common side of two (possibly degenerate) triangles. These triangles make a quadrilateral such that $\alpha$ is one of its diagonal. Then, $\alpha$ is uniquely flipped to another diagonal $\alpha^{\prime}$ of the same quadrilateral, and vice versa. See Figure 15, In particular, the uniqueness of the flip was also shown.

For a given bordered surface $(\mathbf{S}, \mathbf{M})$ it is known that all ideal triangulations are connected by a sequence of flips [Hat91]. In particular, they share the same cardinality 
of arcs. For an ideal triangulation $T$ with $|T|=n$, one can label the $\operatorname{arcs}$ in $T$ by the set $\{1, \ldots, n\}$ as in Figure 14. We call it a labeled ideal triangulation, and we still write it as $T$. In other words, a labeled ideal triangulation $T$ is not simply a set of $n$ arcs; rather, it is an $n$-tuple of arcs $\left(\alpha_{i}\right)_{i=1}^{n}$, where $\alpha_{i}$ is the $\operatorname{arc}$ with label $i$. A flip of an (unlabeled) ideal triangulation induces a flip of a labeled ideal triangulation by preserving the labels of the unflipped arcs. Suppose that $T=\left(\alpha_{i}\right)_{i=1}^{n}$ and $T^{\prime}=\left(\alpha_{i}^{\prime}\right)_{i=1}^{n}$ are labeled ideal triangulations. Then, if $T^{\prime}$ is a flip of $T$ at $\alpha_{k}$, then $T$ is also a flip of $T^{\prime}$ at $\alpha_{k}^{\prime}$. and $\alpha_{i}^{\prime}=\alpha_{i}$ for $i \neq k$. So, we can write them as $T^{\prime}=\mu_{k}(T)$ and $T=\mu_{k}\left(T^{\prime}\right)$, like the mutation in cluster algebras, and call them the fip at $k$.

Definition 5.6. To each labeled ideal triangulation $T=\left(\alpha_{i}\right)_{i=1}^{n}$ of $(\mathbf{S}, \mathbf{M})$ with $|T|=n$, we assign a skew-symmetric matrix $B=B(T)=\left(b_{i j}\right)_{i, j=1}^{n}$, called the (signed) adjacency matrix of $T$, as follows.

(a). The case when neither $\alpha_{i}$ nor $\alpha_{j}$ are inner arcs in $T$. First, for any triangle $\Delta$ in $T$ which is not self-folded, we define

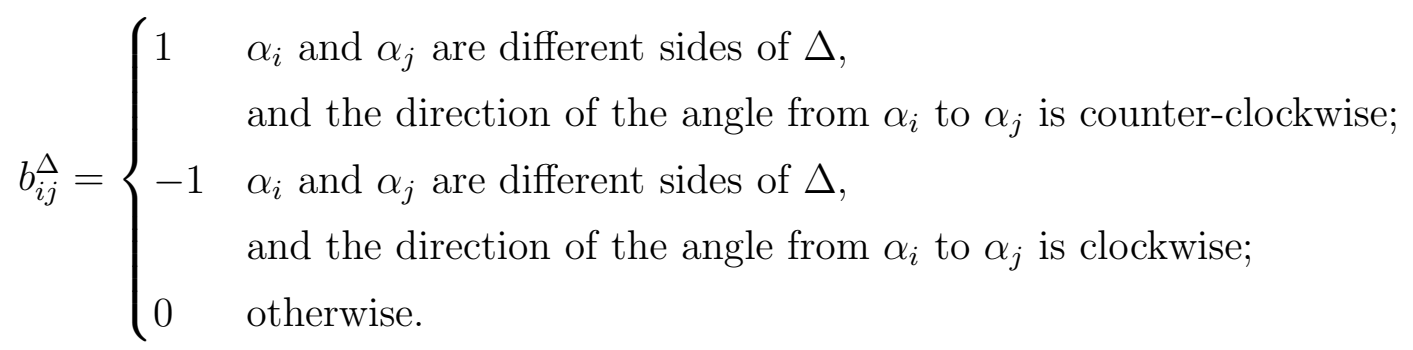

Then, we define

$$
b_{i j}=\sum_{\Delta} b_{i j}^{\Delta},
$$

where the sum runs over all triangles $\Delta$ in $T$ which are not self-folded.

(b). The rest of the case. For an inner $\operatorname{arc} \alpha_{i}$ in $T$, let $\alpha_{\bar{i}}$ be the outer side of the self-folded triangle which $\alpha_{i}$ belongs to. Then, we define

$$
b_{i j}= \begin{cases}b_{\bar{i} j} & \alpha_{i} \text { is an inner arc, and } \alpha_{j} \text { is not; } \\ b_{i \bar{j}} & \alpha_{j} \text { is an inner arc, and } \alpha_{i} \text { is not; } \\ b_{\bar{i} \bar{j}} & \text { both } \alpha_{i} \text { and } \alpha_{j} \text { are inner arcs }\end{cases}
$$

where the right hand side is defined in (5.1).

Example 5.7. For the ideal triangulations in Figure 14, the corresponding skew- 
symmetric matrices and quivers are given as follows.

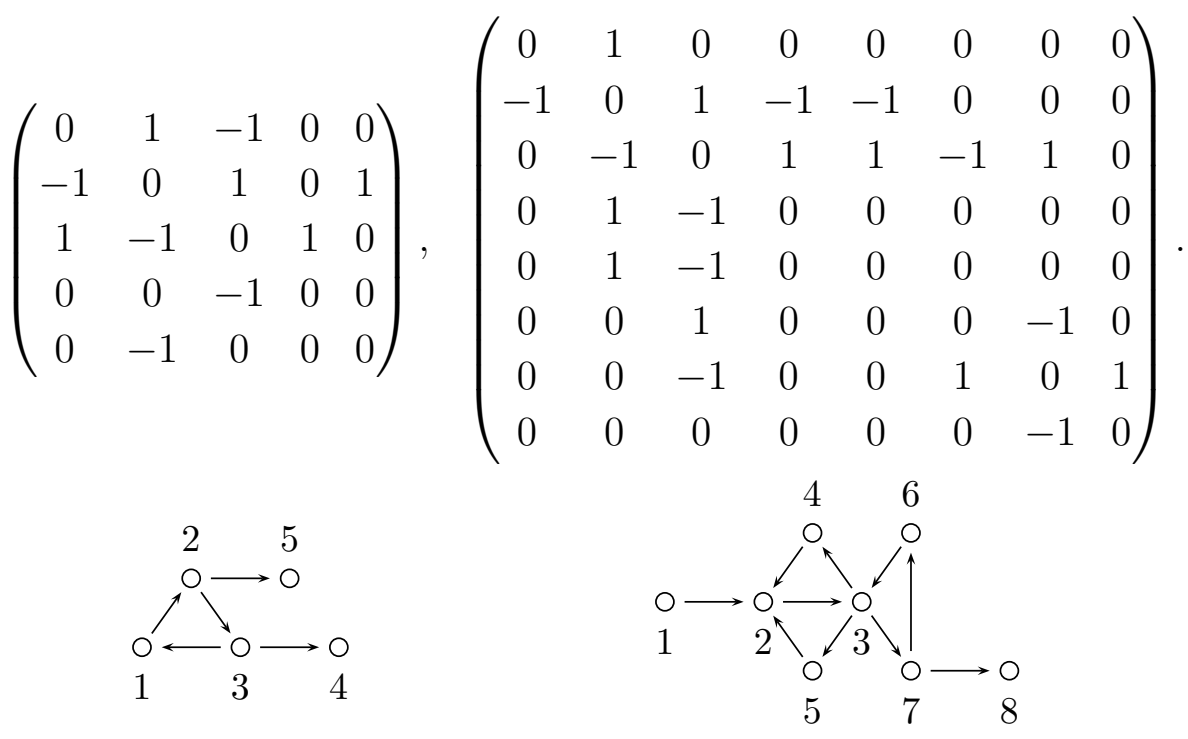
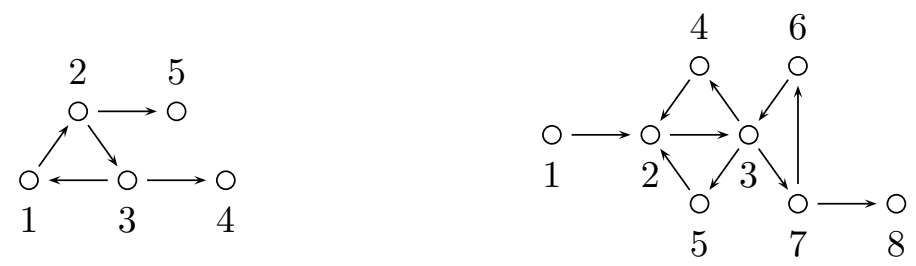

See [FST08, Section 4] for more exotic examples.

The following fact is a key to connect triangulations and cluster algebras.

Theorem 5.8 ([FG07, GSV05]). Let $T$ be a labeled ideal triangulation of (S, M), and let $\alpha_{k}$ not be an inner arc in $T$. Then, $B\left(\mu_{k}(T)\right)=\mu_{k}(B(T))$.

The theorem says that the flip of labeled ideal triangulations and the mutation of the corresponding skew-symmetric matrices (equivalently, quivers) in (4.5) are compatible, if the targeted arc is flippable. However, recall that the inner arcs are not flippable, while skew-symmetric matrices can be mutated to any direction. See Figure 16 for an illuminating example of a digon with a puncture in some ideal triangulation. Fomin, Shapiro, and Thurston [FST08] remedied this discrepancy by introducing the tagged triangulations.

\subsection{Tagged triangulations}

For each arc $\alpha$ in a bordered surface $(\mathbf{S}, \mathbf{M})$, cut $\alpha$ into three pieces and throw out the middle part. The remaining two parts are called the ends of $\alpha$.

Definition 5.9. An arc $\alpha$ in $(\mathbf{S}, \mathbf{M})$ is called a tagged arc if the following conditions are satisfied:

(a). $\alpha$ is not a loop inside which there is exactly one puncture.

(b). Each end of $\alpha$ is tagged in one of two ways, plain or notched such that

- any end with the endpoint on the boundary is tagged plain,

- both ends of a loop are tagged in the same way.

In figures, the plain tags are omitted, while the notched tags are shown by the symbol $\bowtie$, following [FST08]. 


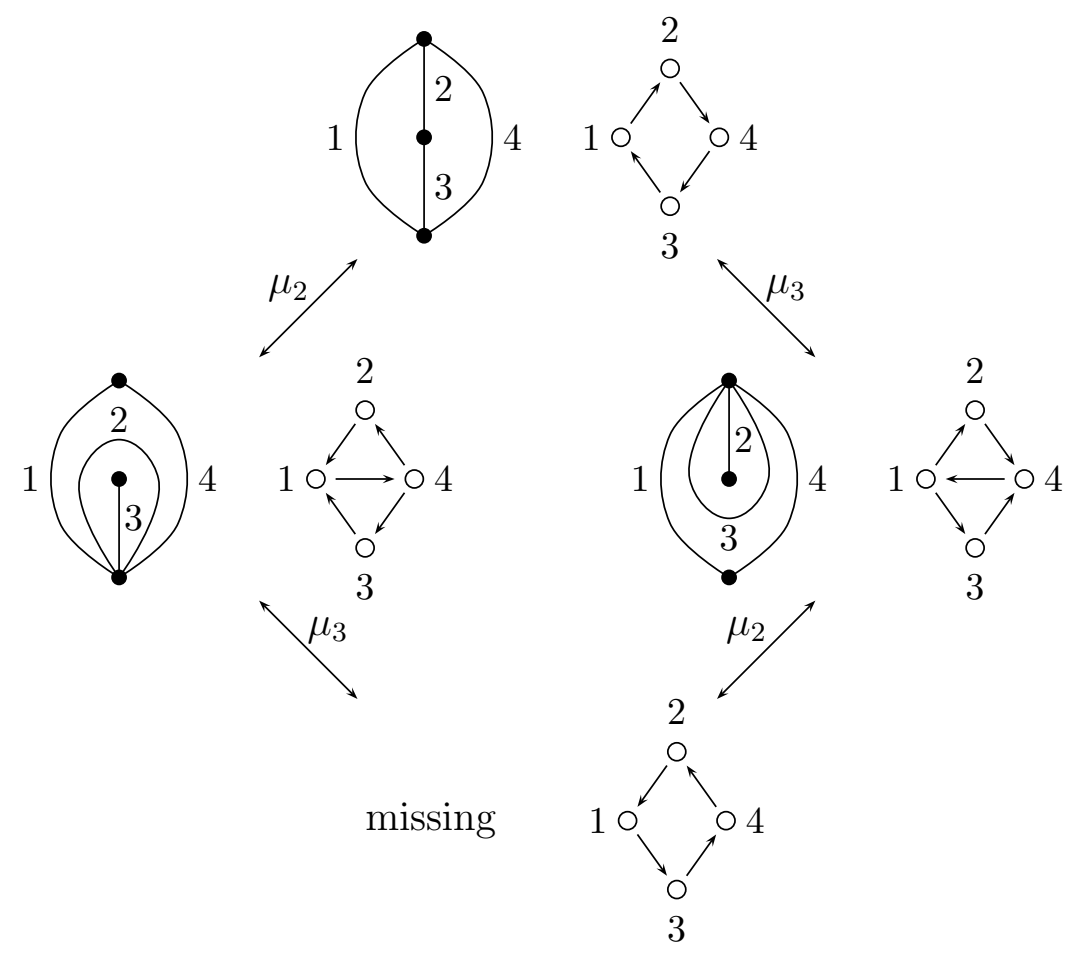

Figure 16. Flips of arcs in a digon with a puncture and mutations of the corresponding quivers.

For example, the loop with label 4 in Figure 14 (b) is not an tagged arc anymore due to the condition (a). If $(\mathbf{S}, \mathbf{M})$ does not have any puncture, then arcs and tagged arcs are the same thing.

Definition 5.10. Two tagged arcs $\alpha$ are $\beta$ are said to be compatible if the following conditions are satisfied:

- the untagged versions of $\alpha$ and $\beta$ are compatible,

- if the untagged versions of $\alpha$ and $\beta$ are distinct, and they share an endpoint $p$, then the ends of $\alpha$ and $\beta$ with endpoint $p$ have the same tag,

- if the untagged versions of $\alpha$ and $\beta$ are identical, then at least one end of $\alpha$ and the corresponding end of $\beta$ have the same tag.

Example 5.11. Suppose that $\alpha, \beta$, and $\gamma$ are three distinct pairwise compatible tagged arcs. Then, their untagged versions are not all identical. To show it, let $p$ and $q$ be their common endpoints, and suppose that the untagged versions of $\alpha$ and $\beta$ are identical. Then, $\alpha$ and $\beta$ have the same tag at one of the ends with endpoint, say, $p$; furthermore, they have the different tags at the end with $q$, since they are distinct tagged arcs. Now suppose further that the untagged versions of $\beta$ and $\gamma$ are identical. If $\beta$ and $\gamma$ have the same tag at the end with $p$, then they have the different tags at the end with $q$. Therefore, $\alpha$ and $\gamma$ are identical as tagged arcs, which is a contradiction. So, $\beta$ and $\gamma$ should have the same tag at the end with $q$, then they have the different tags at the 


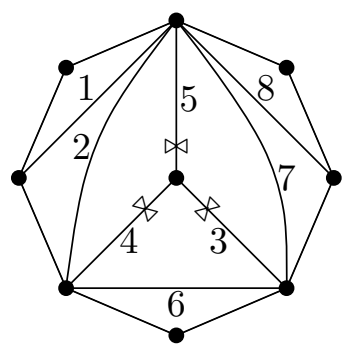

(a)

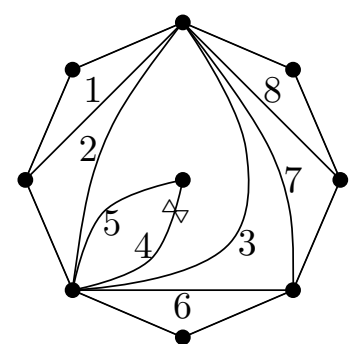

(b)

Figure 17. Examples of labeled tagged triangulations of a polygon with one puncture.
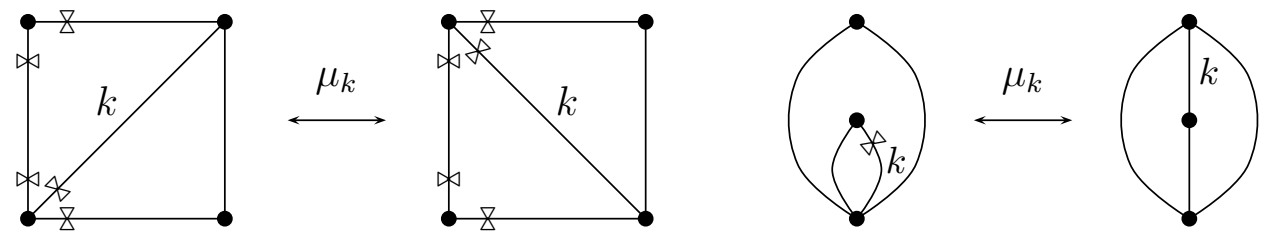

Figure 18. Examples of flips of tagged triangulations

end with $p$. Then, $\alpha$ and $\gamma$ do not have the same tag at both ends. Therefore, $\alpha$ and $\gamma$ is not compatible, which is again a contradiction.

Definition 5.12. An (unlabeled) tagged triangulation $T=\left\{\alpha_{i}\right\}_{i \in I}$ of $(\mathbf{S}, \mathbf{M})$ is a maximal set of distinct pairwise compatible tagged $\operatorname{arcs}$ in $(\mathbf{S}, \mathbf{M})$.

A labeled tagged triangulation is defined in the same way as in the ordinary case. Examples of labeled tagged triangulations are given in Figure 17. Observe that the untagged versions of tagged $\operatorname{arcs} \alpha_{4}$ and $\alpha_{5}$ in (b) are identical.

Definition 5.13. For an unlabeled tagged triangulation $T$ of $(\mathbf{S}, \mathbf{M})$ and a tagged arc $\alpha \in T$, if there is another tagged arc $\alpha^{\prime}$ such that that $T^{\prime}=(T-\{\alpha\}) \cup\left\{\alpha^{\prime}\right\}$ is a tagged triangulation, then $\alpha^{\prime}$ is called a flip of $\alpha$, and $T^{\prime}$ is called a flip of $T$ at $\alpha$. Accordingly, a flip of a labeled tagged triangulation at $k$ is also defined in the same way as in the ordinary case.

The following theorem is the first step to remedy the aforementioned discrepancy.

Theorem 5.14 ([FST08, Theorem 7.9]). Any tagged arc of a tagged triangulation is uniquely flippable.

Example 5.15. (a). Some examples of tagged triangulations are given in Figure 18. Note that the tag of the flipped arc is uniquely determined by the compatibility condition.

(b). In Figure 17, starting from the labeled tagged triangulation in (a), flipping at 3 , then at 5 , one obtains the labeled tagged triangulation in (b).

Next, we assign the adjacency matrix to each labeled tagged triangulation. To do that, we note that for any labeled tagged triangulation $T$, every puncture $p$ of $T$ can be classified in one of the following three types [FST08]. 
- Type 1. All tags of ends with $p$ are plain. For example, consider the one where all notched tags in Figure 17 (a) are replaced with plain.

- Type -1. All tags of ends with $p$ are notched. See Figure 17 (a), for example.

- Type 0. There are both notched and plain tags of ends with $p$. See Figure17(b), for example. In fact, according to Example 5.11, there exists exactly a pair of tagged $\operatorname{arcs} \alpha$ and $\beta$ which end at $p$ such that their untagged versions are identical.

Having this classification in mind, to each labeled tagged triangulation $T=\left(\alpha_{i}\right)_{i=1}^{n}$, we assign a labeled ideal triangulation $T^{\circ}$ as follows:

- Step 1. For each puncture $p$ of type -1 , replace all notched tags of ends with $p$ to plain. For example, in Figure 17 (a), replace the tags of arcs with labels 3, 4, and 5 to plain.

- Step 2. For each puncture $p$ of type 0, do the following replacement.
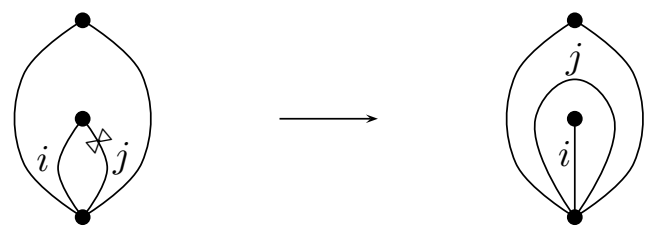

For example, if $T$ is the one in Figure 17 (b), $T^{\circ}$ is given by the one in Figure 14 (b).

Now we extend the definition of the adjacency matrix to the tagged triangulations.

Definition 5.16. To any labeled tagged triangulation $T$, we assign a skew-symmetric matrix $B(T):=B\left(T^{\circ}\right)$, where $T^{\circ}$ is the labeled ideal triangulation associated with $T$ defined above, and we call it the adjacency matrix of $T$.

For example, for $T$ being the one in Figure $17(\mathrm{~b}), B(T)$ is given by the second matrix in Example 5.7.

Finally we have the resolution of the discrepancy.

Theorem 5.17 ([FST08, Lemma 9.7]). For any labeled tagged triangulation $T=\left(\alpha_{i}\right)_{i=1}^{n}$ of $(\mathbf{S}, \mathbf{M})$ and for any $k=1, \ldots, n$, we have $B\left(\mu_{k}(T)\right)=\mu_{k}(B(T))$.

See Figure 19 for an example and compare it with Figure 16.

\subsection{Realization of exchange graph of labeled seeds}

So far, we have concentrated on realizing the exchange matrix part of seeds. We now turn to the realization of the exchange graph of the labeled seeds.

Definition 5.18. The exchange graph of the labeled seeds of a cluster algebra $\mathcal{A}=$ $\mathcal{A}\left(B^{0}, x^{0}, y^{0} ; \mathbb{P}\right)$ is a graph whose vertices are the labeled seeds of $\mathcal{A}$ and a edge are drawn between two vertices if they are related by a mutation.

The following definition is parallel to Definition 4.9. 


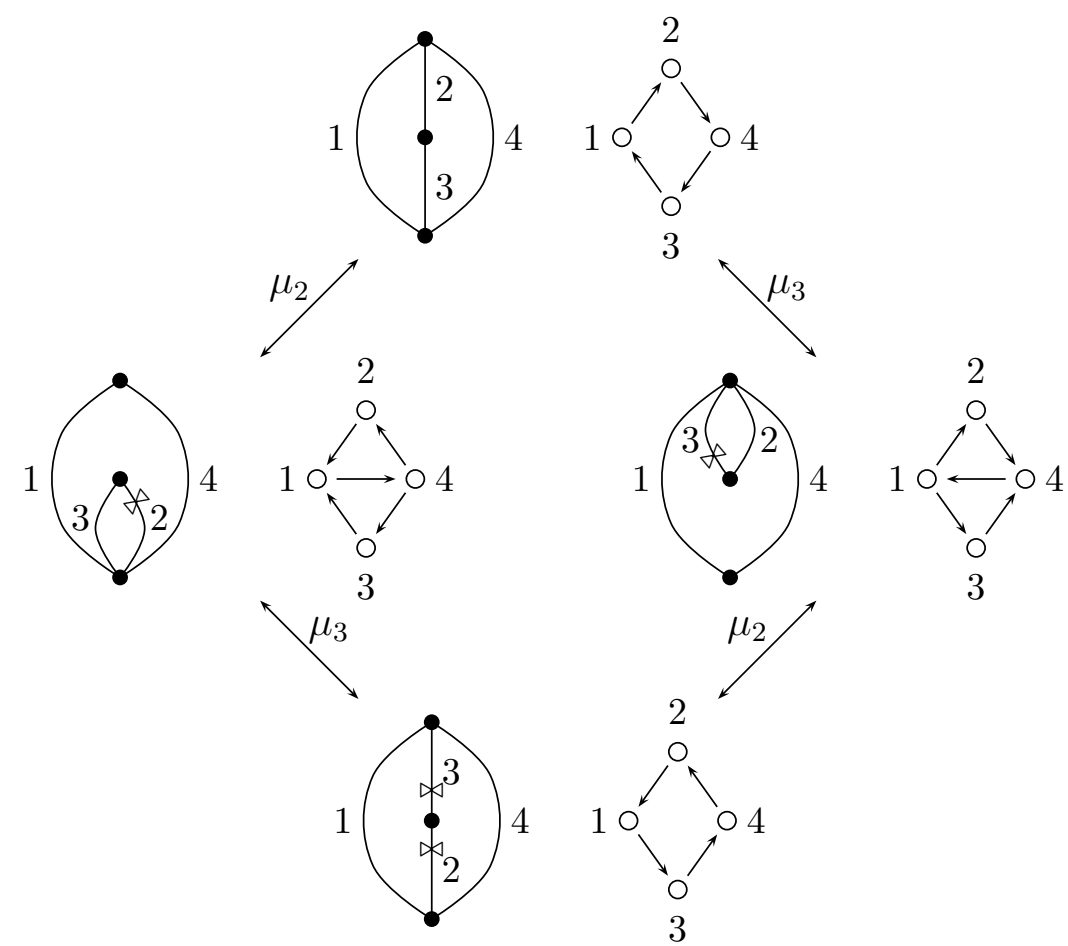

Figure 19. Flips of tagged arcs in a digon with a puncture and mutations of the corresponding quivers.

Definition 5.19. Let $T=\left(\alpha_{i}\right)_{i=1}^{n}$ be a labeled tagged triangulation of a bordered surface $(\mathbf{S}, \mathbf{M})$, and let $\nu$ be a permutation of $\{1, \ldots, n\}$. A mutation sequence $\vec{k}=\left(k_{t}\right)_{t=1}^{N}$ is called a $\nu$-period of $T$ if, for $T^{\prime}=\left(\alpha_{i}^{\prime}\right)_{i=1}^{n}:=\mu_{\vec{k}}(T), \alpha_{\nu(i)}^{\prime}=\alpha_{i}$ holds for any $i$.

Let us fix the initial labeled tagged triangulation $T^{0}=\left(\alpha_{i}^{0}\right)_{i=1}^{n}$ of $(\mathbf{S}, \mathbf{M})$, which is any labeled tagged triangulation. Let $B^{0}=B\left(T^{0}\right)$ be the adjacency matrix of $T^{0}$. Then, we have the associated cluster algebra $\mathcal{A}\left(B^{0}, x^{0}, y^{0} ; \mathbb{Q}_{+}\left(y^{0}\right)\right)$, where the choice of $x^{0}$ is not essential.

Theorem 5.20 (cf. [FST08, Theorem 7.11], [FT12, Theorem 6.1]). Let (B, $x, y)$ and T the ones obtained from $\left(B^{0}, x^{0}, y^{0}\right)$ and $T^{0}$ by the same sequence $\vec{k}$ of mutations/flips. Then, a mutation sequence $\vec{k}$ is a $\nu$-period of $(B, x, y)$ if and only if it is a $\nu$-period of $T$.

Proof. Let us set $\left(B^{\prime}, x^{\prime}, y^{\prime}\right)=\mu_{\vec{k}}(B, x, y)$ and $T^{\prime}=\mu_{\vec{k}}(T)$. Then, Theorem 6.1 of [FT12] tells that $x_{\nu(i)}^{\prime}=x_{i}$ if and only if $\alpha_{\nu(i)}^{\prime}=\alpha_{i}$.

Remark 5.21. Theorem 6.1 of FT12 is the unlabeled version of Theorem 5.20 and here we reformulated (a part of) it for the labeled one with the help of Theorem 4.8,

Example 5.22 (Pentagon relation (2)). The counterpart of the pentagon relation of the seeds in Example 4.10 is given by the mutation sequence of labeled triangulations of a pentagon without a puncture in Figure 20. 


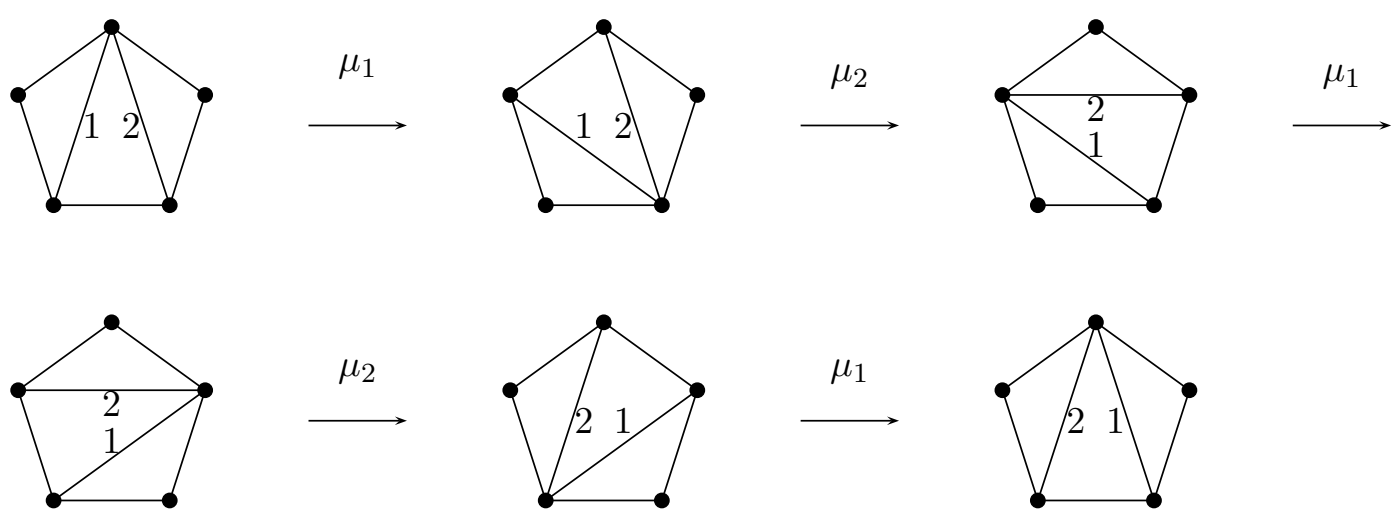

Figure 20. Pentagon relation of labeled triangulations.

Let $\operatorname{LTT}\left(T^{0}\right)$ be the set of all labeled tagged triangulations obtained from the initial labeled tagged triangulation $T^{0}$ by sequences of flips.

By setting $\nu=$ id in Theorem 5.20, we have the following corollary.

Corollary 5.23. There is a bijection $\Psi: \operatorname{Seed}\left(B^{0}, x^{0}, y^{0} ; \mathbb{Q}_{+}\left(y^{0}\right)\right) \rightarrow \operatorname{LTT}\left(T^{0}\right)$ such that $\Psi\left(T^{0}\right)=\left(B^{0}, x^{0}, y^{0}\right)$ and $\Psi$ commutes with flips/mutations.

In other words, the exchange graph of $\mathcal{A}\left(B^{0}, x^{0}, y^{0} ; \mathbb{Q}_{+}\left(y^{0}\right)\right)$ is identified with the exchange graph of the labeled tagged triangulations in $\operatorname{LTT}\left(T^{0}\right)$ by flips.

To present a general statement on $\operatorname{LTT}\left(T^{0}\right)$, we need to exclude some "exceptional" bordered surfaces.

Definition 5.24. A bordered surface $(\mathbf{S}, \mathbf{M})$ is said to be generic if it is not one of the following:

- an once-punctured digon

- an unpunctured annulus with one marked point on each boundary component

- an once-punctured torus

This definition is motivated by the following property which holds only for generic bordered surfaces.

Lemma 5.25. Let $(\mathbf{S}, \mathbf{M})$ be a generic bordered surface, let $T=\left(\alpha_{i}\right)_{i=1}^{n}$ be any labeled tagged triangulation of $(\mathbf{S}, \mathbf{M})$, and let $B=B(T)$. Then, for any pair of distinct indices $i, j \in\{1, \ldots, n\}$, there is a sequence of indices $i_{0}=i, i_{1}, \ldots, i_{r}=j$ such that $\left|b_{i_{s} i_{s+1}}\right|=1$ for any $s=0, \ldots, r-1$.

Proof. This is an immediate consequence of the construction of ideal triangulations and the associated adjacency matrices by "puzzle pieces" and "blocks" in [FST08, Theorem 13.3].

Proposition 5.26 (cf. [FT12, Proposition 7.10]). Let (S, M) be a generic bordered surface. 


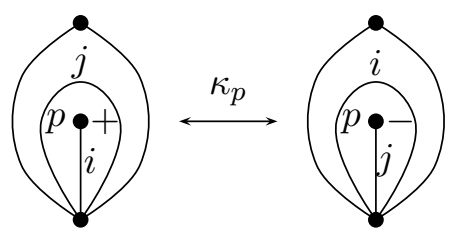

Figure 21. Pop at a puncture $p$ inside a self-folded triangle.

(a). If $(\mathbf{S}, \mathbf{M})$ is not a closed surface with exactly one puncture, then $\operatorname{LTT}\left(T^{0}\right)$ consists of all labeled tagged triangulations of $(\mathbf{S}, \mathbf{M})$.

(b). If $(\mathbf{S}, \mathbf{M})$ is a closed surface with exactly one puncture, then $\mathrm{LTT}\left(T^{0}\right)$ consists of all labeled tagged triangulations of $(\mathbf{S}, \mathbf{M})$ whose arcs are tagged in the same way as the arcs of $T^{0}$.

Proof. The unlabeled version of the statement is true by [FT12, Proposition 7.10] (including the nongeneric case). On the other hand, thanks to Lemma 5.25, for any labeled tagged triangulation $T$ of $(\mathbf{S}, \mathbf{M})$, one can exchange the labels of any pair of arcs of $T$ by repeatedly applying the sequence of flips for a pentagon in Figure 20 (and flips in Figure 19 if necessary). Thus, the statement is also true for the labeled one.

\subsection{Reformulation by signed triangulations}

Let us explain the notion of signed triangulations recently introduced by [LF12, [BS13]. It is nothing but an alternative way of expressing tagged triangulations, but it involves the operation called pop.

Definition 5.27. A labeled signed triangulation of a bordered surface $(\mathbf{S}, \mathbf{M})$ is a pair $T_{\sigma}=(T, \sigma)$ such that $T$ is a labeled ideal triangulation of $(\mathbf{S}, \mathbf{M})$ and $\sigma$ is a sign function from the set of the punctures in $(\mathbf{S}, \mathbf{M})$ to the sign set $\{+,-\}=\{1,-1\}$. The sign $\sigma(p)$ at $p$ is denoted by $\sigma_{p}$.

Let $T_{\sigma}$ be a labeled signed triangulation. For a puncture $p$ inside a self-folded triangle of $T_{\sigma}$, we define the operation $\kappa_{p}$ as illustrated in Figure 21, and we call it the pop at $p$. It is important that the labels $i$ and $j$ are interchanged by a pop. (It was first introduced by GMN13] without sign.) Let us introduce an equivalence relation, called the pop-equivalence, among the labeled signed triangulations of $(\mathbf{S}, \mathbf{M})$ such that $T_{\sigma} \sim T_{\sigma^{\prime}}^{\prime}$ if they are related by a finite sequence of pops, including the empty sequence. The equivalence class of $T_{\sigma}$ is denoted by $\left[T_{\sigma}\right]$ and called the pop-equivalence class of $T_{\sigma}$.

Proposition 5.28 ([LF12, BS13]). There is a natural one-to-one correspondence between the labeled tagged triangulations of $(\mathbf{S}, \mathbf{M})$ and the pop-equivalence classes of the labeled signed triangulations of $(\mathbf{S}, \mathbf{M})$.

The correspondence is given as follows. A labeled tagged triangulation $T$ is identified with the pop-equivalence class $\left[T_{\sigma}^{\prime}\right]$, where its representative $T_{\sigma}^{\prime}=\left(T^{\prime}, \sigma\right)$ 


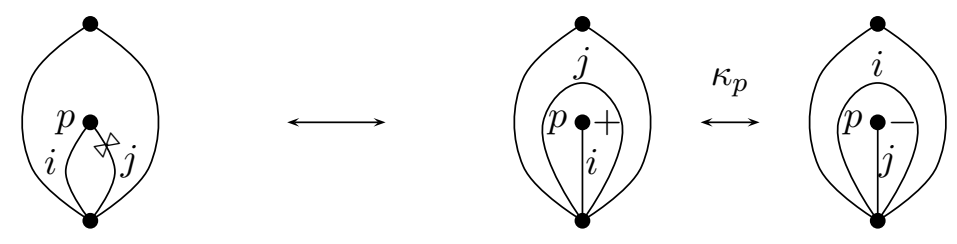

Figure 22. Two representatives of a labeled tagged triangulation inside a digon with a puncture by labeled signed triangulations.

is obtained from $T$ by doing firstly the following operation for each puncture $p$ in $T$, and then removing the the tags of all tagged arcs:

- if $p$ is of type 1 (as defined in Section 5.2), assign the sign $\sigma_{p}=+$,

- if $p$ is of type -1 , assign the sign $\sigma_{p}=-$,

- if $p$ is of type 0 , we may do one of two ways (see Figure 22):

- (i) replace the notched tagged arc ending at $p$ with the loop surrounding $p$, and assign the sign $\sigma_{p}=+$, or

- (ii) replace the plain tagged arc ending at $p$ with the loop surrounding $p$, and assign the sign $\sigma_{p}=-$.

Two choices are exactly connected by the pop $\kappa_{p}$, thus they define the same popequivalence class.

Using this new presentation, our familiar example of flips inside a digon with a puncture looks as in Figure 23,

\subsection{Local rescaling and signed pops of extended seeds}

Here we point out a hidden symmetry of the exchange relation (4.22) called the local rescaling. This symmetry presents when the seeds admit surface realization. Using it, we define the signed pops for extended seeds.

Let $T$ be a labeled ideal triangulation of a bordered surface $(\mathbf{S}, \mathbf{M})$, and let $B$ be the adjacency matrix of $T$. We concentrate on a seed $(B, x, y)$ with coefficients in $\operatorname{Trop}\left(y^{0}\right)$, though the argument can be applicable to a more general situation. Let $\mathcal{P}$ be the set of the punctures of $(\mathbf{S}, \mathbf{M})$.

Definition 5.29. For any puncture $p \in \mathcal{P}$ and any nonzero rational number $c$, we call the following operation for each $x$-variable $x_{i}$ of $x$ the local rescaling at $p$ by the constant $c$ :

- If the corresponding arc $\alpha_{i}$ ends at the puncture $p$, then multiply $c$ for $x_{i}$.

- If the corresponding arc $\alpha_{i}$ is the outer edge of a self-folded triangle with $p$ inside it, then multiply $c^{-1}$ for $x_{i}$.

- Otherwise, leave $x_{i}$ as it is.

Lemma 5.30. For any $k$, the factor $\hat{y}_{k}$ in (4.22) is invariant under the local rescaling. 


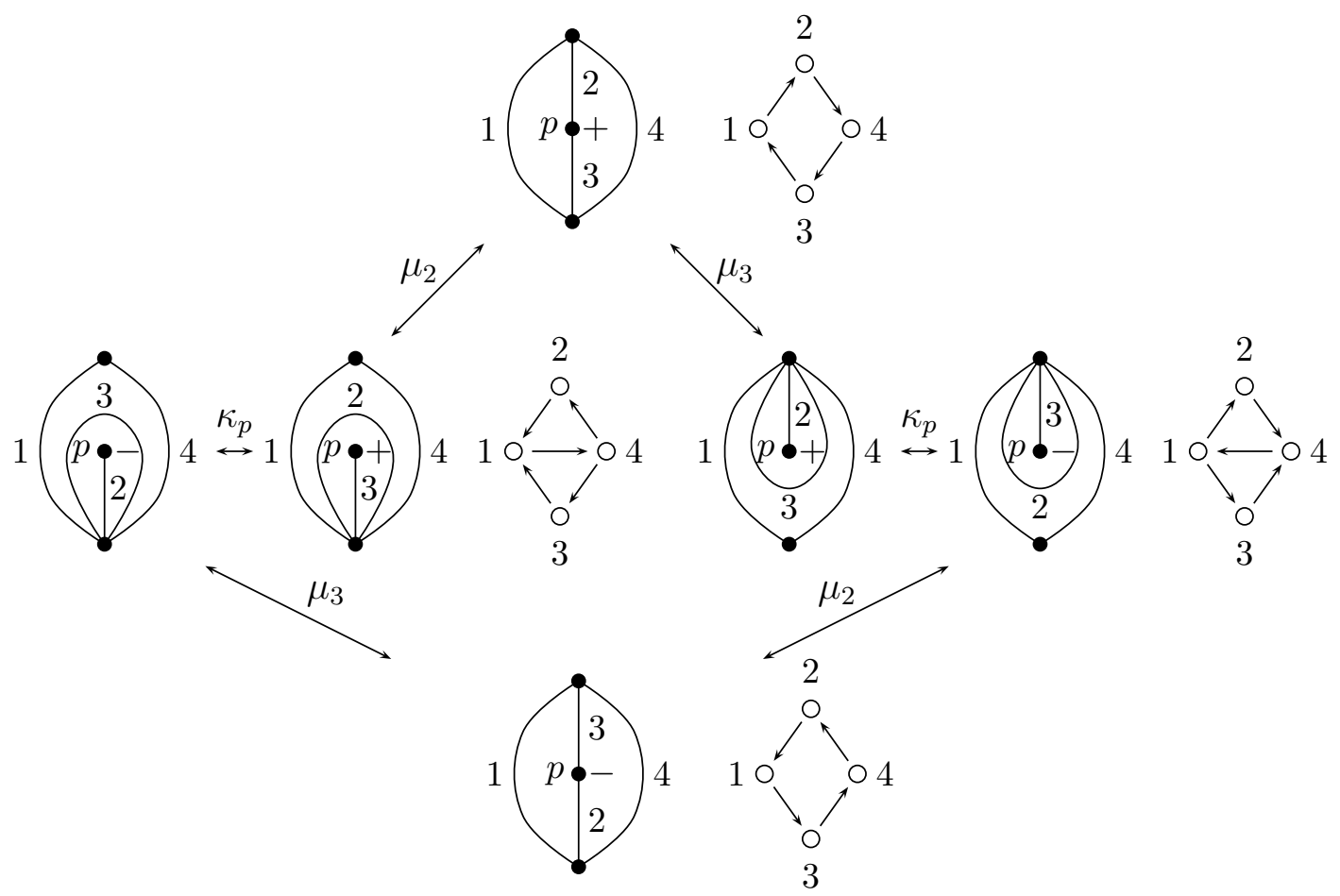

Figure 23. Flips and pops of labeled signed triangulations of a digon with a puncture.

Proof. This can be verified by case-check of configurations involving the puncture $p$ and the $\operatorname{arc} \alpha_{k}$.

Suppose that the arc $\alpha_{k}$ of $T$ is flippable. We apply the flip $T^{\prime}=\mu_{k}(T)$ and the signed mutation $\left(B^{\prime}, x^{\prime}, y^{\prime}\right)=\mu_{k}^{(\varepsilon)}(B, x, y)$ in Section 4.6, respectively. (Note that $B^{\prime}=B\left(T^{\prime}\right)$ holds for any $\varepsilon$.) Then, the local rescaling is defined also for $x^{\prime}$ by $T^{\prime}$.

Proposition 5.31. The signed mutation $\mu_{k}^{(\varepsilon)}$ and the local rescaling at $p$ by c commute with each other.

Proof. By Lemma 5.30, it is enough to show that $x_{k}^{\prime}$ and $x_{k}^{-1} \prod_{j=1}^{n} x_{j}^{\left[-\varepsilon b_{j k}\right]_{+}}$in (4.22) rescale by the same factor. This can be verified by case-check.

Having the above property in mind, we introduce the notion of extended seeds and their signed pops. Recall that our cluster algebra is a $\mathbb{Z} \mathbb{P}$-subalgebra of the ambient field $\mathbb{Q P}(w)$ for some variables $w=\left(w_{i}\right)$ with $\mathbb{P}=\operatorname{Trop}\left(y^{0}\right)$. We introduce a $\mathcal{P}$-tuple of new algebraically independent variables $\tilde{y}^{0}=\left(\tilde{y}_{p}^{0}\right)_{p \in \mathcal{P}}$. Let $\tilde{\mathbb{Q}}:=\mathbb{Q}\left(\tilde{y}^{0}\right)$ be the rational function filed of $\tilde{y}^{0}$ over $\mathbb{Q}$. In particular,

$$
1-\tilde{y}_{p}^{0}, 1-\left(\tilde{y}_{p}^{0}\right)^{-1},\left(1-\tilde{y}_{p}^{0}\right)^{-1},\left(1-\left(\tilde{y}_{p}^{0}\right)^{-1}\right)^{-1} \in \tilde{\mathbb{Q}} .
$$

We extend the ambient field $\mathbb{Q P}(w)$ to $\tilde{\mathbb{Q}} \mathbb{P}(w)$.

Let $B=B(T)$ be the adjacency matrix of a labeled ideal triangulation $T$ of a bordered surface $(\mathbf{S}, \mathbf{M})$. We extend a labeled seed $(B, x, y)$ to an labeled extended seed 
$(B, x, y, \tilde{y})$, where $\tilde{y}=\left(\tilde{y}_{p}\right)_{p \in \mathcal{P}}, \tilde{y}_{p} \in\left\{\tilde{y}_{p}^{0},\left(\tilde{y}_{p}^{0}\right)^{-1}\right\}$. We call $\tilde{y}_{p}$ the coefficient at $p$, or simply a $\tilde{y}$-variable. In particular, we extend the initial seed $\left(B^{0}, x^{0}, y^{0}\right)$ to the initial extended seed $\left(B^{0}, x^{0}, y^{0}, \tilde{y}^{0}\right)$, where $\tilde{y}^{0}=\left(\tilde{y}_{p}^{0}\right)_{p \in \mathcal{P}}$ are the ones as above. Then, we extend the signed mutation of $\left(B^{\prime}, x^{\prime}, y^{\prime}\right)=\mu_{k}^{(\varepsilon)}(B, x, y)$ in (4.20) and (4.22) to the signed mutation $\left(B^{\prime}, x^{\prime}, y^{\prime}, \tilde{y}^{\prime}\right)=\mu_{k}^{(\varepsilon)}(B, x, y, \tilde{y})$ (for labeled extended seeds) in a trivial way by keeping (4.20) and (4.22) and setting $\tilde{y}^{\prime}=\tilde{y}$.

Finally, for a puncture $p$ inside a self-folded triangle in $T$, we define the signed pop $\left(B^{\prime}, x^{\prime}, y^{\prime}, \tilde{y}^{\prime}\right)=\kappa_{p}^{(\varepsilon)}(B, x, y, \tilde{y})$ at $p$ with sign $\varepsilon$ (for labeled extended seeds) by setting $B^{\prime}=B, y^{\prime}=y$, and

$$
\begin{aligned}
& \tilde{y}_{q}^{\prime}= \begin{cases}\tilde{y}_{p}^{-1} & q=p \\
\tilde{y}_{q} & q \neq p,\end{cases} \\
& x_{i}^{\prime}= \begin{cases}\left(1-\tilde{y}_{p}^{\varepsilon}\right) x_{i_{p}} & i=i_{p} \\
\left(1-\tilde{y}_{p}^{\varepsilon}\right)^{-1} x_{j_{p}} & i=j_{p} \\
x_{i} & i \neq i_{p}, j_{p},\end{cases}
\end{aligned}
$$

where $i_{p}$ and $j_{p}$ are the labels of the inner and outer arcs of the self-folded triangle around $p$ in $T_{s, a}$. The signed pop $\kappa_{p}^{(\varepsilon)}$ acts on $x$ as the local rescaling at $p$ by the constant $1-\tilde{y}_{p}{ }^{\varepsilon}$ (in the extended field $\tilde{\mathbb{Q}}$ ). It is easy to see that $\kappa_{p}^{(\varepsilon)}$ is not an involution, but $\kappa_{p}^{(+)}$and $\kappa_{p}^{(-)}$are inverse to each other.

\section{Mutation of Stokes graphs}

In this section we study the mutation of Stokes graphs, which is purely geometric. We introduce Stokes triangulations, and their signed flips and pops. They effectively control the mutation of Stokes graphs; moreover, they give a bridge between the exact WKB analysis and cluster algebra theory. We also introduce the simple paths and the simple cycles of a Stokes graph, and give their mutation formulas.

\subsection{Stokes triangulations, signed flips, and signed pops}

To work with the mutation of Stokes graphs, it is natural to extend the notions of bordered surfaces and their ideal triangulations.

Definition 6.1. For a bordered surface $(\mathbf{S}, \mathbf{M})$, let $m$ be the total number of triangles in any ideal triangulation $T$ of $(\mathbf{S}, \mathbf{M})$, where $m$ does not depend on $T$. Accordingly, we introduce a set $\mathbf{A}$ consisting of $m$ points of $\mathbf{S}$ such that $\mathbf{A} \cap \partial \mathbf{S}=\mathbf{A} \cap \mathbf{M}=\emptyset$. We call $a \in \mathbf{A}$ a midpoint (of a triangle), and in figures it will be shown by a cross. For brevity, we still call $(\mathbf{S}, \mathbf{M}, \mathbf{A})$ a bordered surface.

Definition 6.2. An arc $\alpha$ in a bordered surface $(\mathbf{S}, \mathbf{M}, \mathbf{A})$ is a curve in $\mathbf{S} \backslash \mathbf{A}$ satisfying the four conditions in Definition 5.3. Each arc $\alpha$ is considered up to isotopy in the class of such curves. 

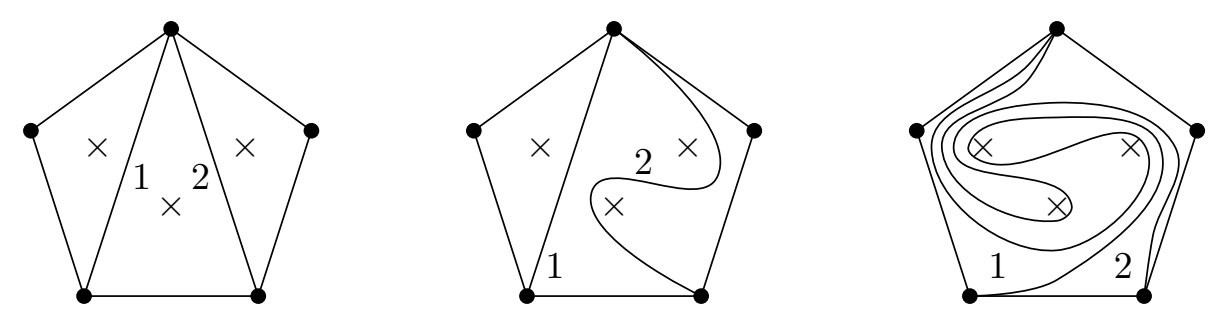

Figure 24. Examples of labeled Stokes triangulations of a pentagon.

When we consider an $\operatorname{arc} \alpha$ in $(\mathbf{S}, \mathbf{M}, \mathbf{A})$, it is sometimes convenient to regard it as an arc in $(\mathbf{S}, \mathbf{M})$ by forgetting the midpoints. In that case we write the latter arc as $\tilde{\alpha}$ to avoid confusion.

Definition 6.3. An $n$-tuple $T=\left(\alpha_{i}\right)_{i=1}^{n}$ of $\operatorname{arcs}$ in $(\mathbf{S}, \mathbf{M}, \mathbf{A})$ is called a labeled Stokes triangulation of $(\mathbf{S}, \mathbf{M}, \mathbf{A})$ if the following conditions are satisfied:

- The $\operatorname{arcs} \alpha_{1}, \ldots, \alpha_{n}$ in $(\mathbf{S}, \mathbf{M}, \mathbf{A})$ are pairwise compatible (in the same sense as before but considered in the isotopy classes for $\operatorname{arcs}$ in $(\mathbf{S}, \mathbf{M}, \mathbf{A})$ ).

- The $n$-tuple $\tilde{T}=\left(\tilde{\alpha}_{i}\right)_{i=1}^{n}$ of arcs in $(\mathbf{S}, \mathbf{M})$ yields a labeled ideal triangulation of $(\mathbf{S}, \mathbf{M})$.

- Every triangle of $T$ contains exactly one midpoint.

Some examples of labeled Stokes triangulations of a pentagon are given in Figure 24. Three triangulations therein are distinct as labeled Stokes triangulations, but they are identical as labeled ideal triangulations by forgetting the midpoints.

For a labeled Stokes triangulation $T=\left(\alpha_{i}\right)_{i=1}^{n}$ of $(\mathbf{S}, \mathbf{M}, \mathbf{A})$, an $\operatorname{arc} \alpha_{k}$ is said to be flippable if it is not an inner arc of $T$. However, unlike the case of labeled ideal triangulations of $(\mathbf{S}, \mathbf{M})$, the "flip" of a flippable arc $\alpha_{k}$ is not unique. In fact, there are infinitely many choices of doing "flip" of a flippable arc $\alpha_{k}$ to obtain a new Stokes triangulation. It is not difficult to see that they are generated by the following two elementary flips.

Definition 6.4. For a labeled Stokes triangulation $T=\left(\alpha_{i}\right)_{i=1}^{n}$ of $(\mathbf{S}, \mathbf{M}, \mathbf{A})$, a flippable arc $\alpha_{k}$ of $T$, and a sign $\varepsilon \in\{+,-\}$, the signed flip $T^{\prime}=\mu_{k}^{(\varepsilon)}(T)$ at $k$ with sign $\varepsilon$ is a labeled Stokes triangulation of (S, M, A) obtained from $T$ by replacing the $\operatorname{arc} \alpha_{k}$ with the one in Figure 25. Namely, the new arc $\alpha_{k}^{\prime}$ is obtained from $\alpha_{k}$ by sliding each end point of $\alpha_{k}$ along an edge of the surrounding quadrilateral of $\alpha_{k}$ clockwise for $\varepsilon=+$ and anticlockwise for $\varepsilon=-$.

Clearly, $\mu_{k}^{(\varepsilon)}$ is not an involution any more, but $\mu_{k}^{(+)}$and $\mu_{k}^{(-)}$are inverse to each other. Figure 26 demonstrates how a sequence of signed flips act on a quadrilateral.

We also introduce the signed pops for labeled Stokes triangulations.

Definition 6.5. For a labeled Stokes triangulation $T$ of $(\mathbf{S}, \mathbf{M}, \mathbf{A})$, a puncture $p$ inside a self-folded triangle in $T$, and a sign $\varepsilon \in\{+,-\}$, the signed pop $T^{\prime}=\kappa_{p}^{(\varepsilon)}(T)$ at $p$ with sign $\varepsilon$ is a labeled Stokes triangulation of $(\mathbf{S}, \mathbf{M}, \mathbf{A})$ obtained from $T$ by replacing the 


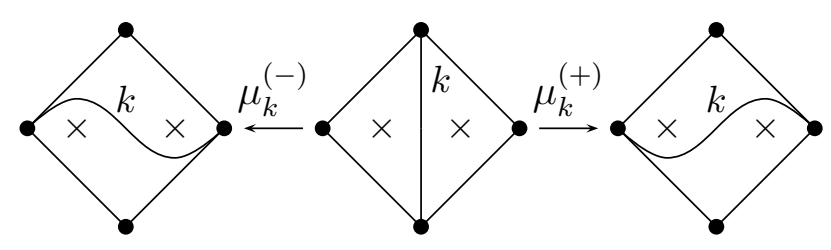

Figure 25. Signed flips of labeled Stokes triangulations.

inner arc of the self-folded triangle around $p$ with the one in Figure 27, then exchanging the labels of the inner and outer arcs thereof. Namely, if $\alpha_{i}$ and $\alpha_{j}$ is the inner and outer arcs of $T$, then $\alpha_{i}^{\prime}=\alpha_{j}$, and $\alpha_{j}^{\prime}$ is obtained from $\alpha_{i}$ by sliding the outside end point of $\alpha_{i}$ along the surrounding monogon clockwise for $\varepsilon=+$ and anticlockwise for $\varepsilon=-$.

Again, $\kappa_{p}^{(\varepsilon)}$ is not an involution any more, but $\kappa_{p}^{(+)}$and $\kappa_{p}^{(-)}$are inverse to each other. Figure 28 demonstrates how a sequence of signed pops act.

The signed flips and the signed pops of Stokes triangulations are supposed to be the counterparts of the signed mutations and the signed pops of extended seeds in Section 5.5. To be more precise, we have the following conjecture, which naturally extends Corollary 5.23 and Proposition 5.26, (We thank Yuuki Hirako for the discussion.)

Conjecture 6.6. Let $T^{0}$ be any labeled Stokes triangulation of (S, M, A), and let $B^{0}=B\left(T^{0}\right)$.

(i) Let $\operatorname{LST}\left(T^{0}\right)$ be the set of all labeled Stokes triangulations obtained from the initial labeled Stokes triangulation $T^{0}$ of (S, M, A) by sequences of signed flips and signed pops, and let $\operatorname{Seed}\left(B^{0}, x^{0}, y^{0}, \tilde{y}^{0}\right)$ be the set of all labeled extended seeds which are mutation-equivalent to the initial one $\left(B^{0}, x^{0}, y^{0}, \tilde{y}^{0}\right)$ by signed mutations and signed pops. Then, there is a bijection $\Psi: \operatorname{LST}\left(T^{0}\right) \rightarrow \operatorname{Seed}\left(B^{0}, x^{0}, y^{0}, \tilde{y}^{0}\right)$ such that $\Psi\left(T^{0}\right)=\left(B^{0}, x^{0}, y^{0}, \tilde{y}^{0}\right)$ and $\Psi$ commutes with signed flips/mutations and signed pops.

(ii) Assume that (S, M) is generic and not a closed surface with exactly one puncture. Then, $\mathrm{LST}\left(T^{0}\right)$ consists of all labeled Stokes triangulations of $(\mathbf{S}, \mathbf{M}, \mathbf{A})$.

In the rest of paper we do not rely on this conjecture, but having it in mind will be useful.

Remark 6.7. The notions of Stokes triangulations and signed flips (for surfaces without punctures) also appear recently in Qiu14 in the study of the spherical twists on 3Calabi-Yau categories.

\subsection{Construction of Stokes triangulation from Stokes graph}

Let $G=G(\phi)$ be the Stokes graph of a quadratic differential $\phi$ on a compact Riemann surface $\Sigma$. The classification of the Stokes regions of $G$ was given in Section 2.6] under Assumptions 2.3 and 2.14. To be more specific, if $G$ is saddle-free, a degenerate ring domain does not appear. Thus, any Stokes region of $\phi$ falls into one of the following three 


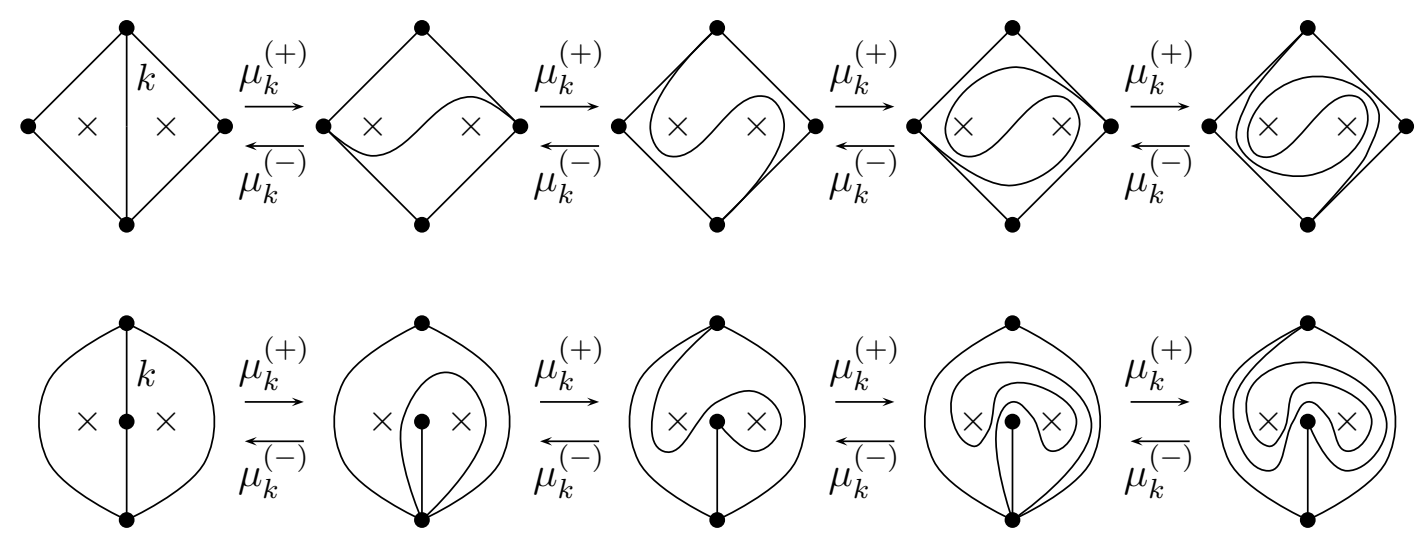

Figure 26. Examples of sequences of signed flips.

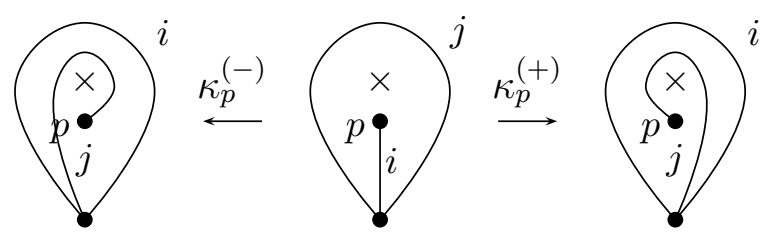

Figure 27. Signed pops of labeled Stokes triangulations.

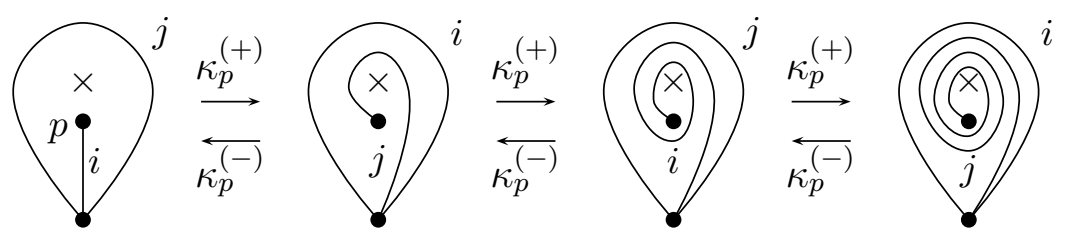

Figure 28. Examples of sequences of signed pops.

patterns, which are depicted in Figure 29 [BS13, Section 3.4], where we split horizontal strips in two patterns:

(a). Regular horizontal strip. This is a generic case. The Stokes region is inside a quadrilateral with two simple zeros $q_{1}, q_{2}$ and two poles $p_{1}, p_{2}$ of orders $m_{1}, m_{2} \geq 2$. The poles $p_{1}, p_{2}$ may coincide.

(b). Degenerate horizontal strip. This may be regarded as the folding of the two edges $q_{1} p_{2}$ and $q_{2} p_{2}$ in the case (a). The orders of poles $p_{1}$ and $p_{2}$ are $m_{1} \geq 2$ and $m_{2}=2$, respectively.

(c). Half plane. This occurs only for a pole $p_{1}$ with order $m_{1} \geq 3$.

Note that the pictures in Figure 29 are schematic ones, and actual trajectories entering in a pole should obey the local property in Section 2.6, depending on the order of the pole. The dashed arc is a representative of the isotopy class of trajectories inside the Stokes region.

Let us introduce labeling of a saddle-free Stokes graph. 


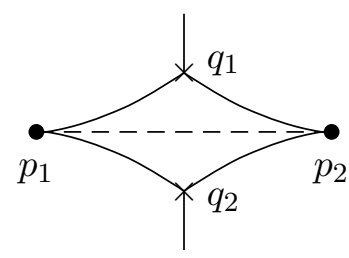

(a) regular horizontal strip

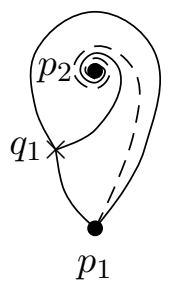

(b) degenerate horizontal strip

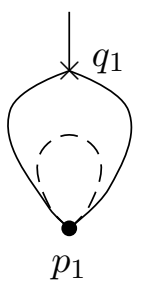

(c) half plane

Figure 29. Patterns of Stokes regions for saddle-free Stokes graph. The dashed arc is a representative of the isotopy class of trajectories inside the Stokes region.

Definition 6.8. Let $G=G(\phi)$ be the Stokes graph of a saddle-free quadratic differential $\phi$ on $\Sigma$. We put labels, say, $D_{1}, \ldots, D_{n}$ for the Stokes regions of $G$ which are (regular or degenerate) horizontal strips. It is called a labeled Stokes graph, and denoted by the same symbol $G$. (We do not put labels for the Stokes regions which are half planes.)

To each labeled Stokes graph $G=G(\phi)$, we will assign a labeled Stokes triangulation $T$ of a certain bordered surface $(\mathbf{S}, \mathbf{M}, \mathbf{A})$. We follow the construction of an ideal triangulation by [KT05, GMN13, BS13], which is automatically upgraded to a labeled Stokes triangulation.

Step 1. Construction of the bordered surface $(\mathbf{S}, \mathbf{M}, \mathbf{A})$. Let $p_{1}, \ldots, p_{r}$ be the double poles of $\phi$, and $p_{1}^{\prime}, \ldots, p_{s}^{\prime}$ be the poles of orders $m_{1}, \ldots, m_{s} \geq 3$ of $\phi$. Then, the bordered surface $\mathbf{S}=\mathbf{S}(\phi)$ is obtained from $\Sigma$ by cutting out a small hole around each $p_{i}^{\prime}$ such that no other poles and zeros of $\phi$ are removed. Let $B_{i}$ denote the resulting boundary component for $p_{i}^{\prime}$. To each $B_{i}$ we put $m_{i}-2$ marked points. Then, the set of the marked points $\mathbf{M}=\mathbf{M}(\phi)$ consists of these marked points at boundaries and the double poles $p_{1}, \ldots, p_{r}$. (Thus, $p_{1}, \ldots, p_{r}$ are the punctures of $(\mathbf{S}, \mathbf{M})$.) Also, the set of the midpoints $\mathbf{A}=\mathbf{A}(\phi)$ are given by the zeros of $\phi$.

Step 2. Construction of the labeled Stokes triangulation $T$ of $(\mathbf{S}, \mathbf{M}, \mathbf{A})$. For each Stokes region $D$ which is a (regular or degenerate) horizontal strip, choose any representative $\beta$ of trajectories in $D$ up to isotopy. We identify $\beta$ with an $\operatorname{arc} \alpha$ of $(\mathbf{S}, \mathbf{M}, \mathbf{A})$ in the following way. If the poles $p_{1}$ and $p_{2}$ in Figure 29 (a) or (b) are double poles, then the arc $\alpha$ is the one connecting $p_{1}$ and $p_{2}$ therein. If $p_{i}$ is a pole of order $m \geq 3$, we do the following modification: We identify the $m-2$ marked points at the boundary component for $p_{i}$ with the $m-2$ tangent directions of trajectories around $p_{i}$, keeping the clockwise order. Then the arc $\alpha$ ends at the marked point at the boundary component for $p_{i}$ corresponding to the tangent direction of $\beta^{\prime}$ as in Figure 30 .

Let us collect the resulting arcs $\alpha_{1}, \ldots, \alpha_{n}$ corresponding to the Stokes regions $D_{1}$, $\ldots, D_{n}$ which are horizontal strips. Let us show that $T=T(\phi)=\left(\alpha_{i}\right)_{i=1}^{n}$ is a labeled Stokes triangulation of $(\mathbf{S}, \mathbf{M}, \mathbf{A})$.

Proposition 6.9 ([BS13, Lemma 10.1]). The $n$-tuple $\tilde{T}=\left(\tilde{\alpha}_{i}\right)_{i=1}^{n}$ of arcs in $(\mathbf{S}, \mathbf{M})$ is a labeled ideal triangulation of $(\mathbf{S}, \mathbf{M})$. 

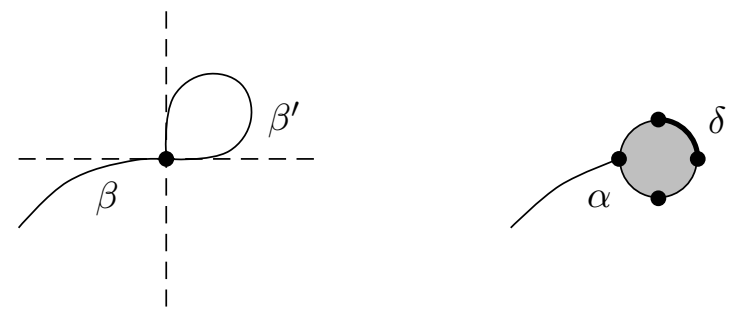

Figure 30. Identification of trajectories around a pole of order $m \geq 3$ with $\operatorname{arcs}$ and edges. The case $m=6$ is shown.
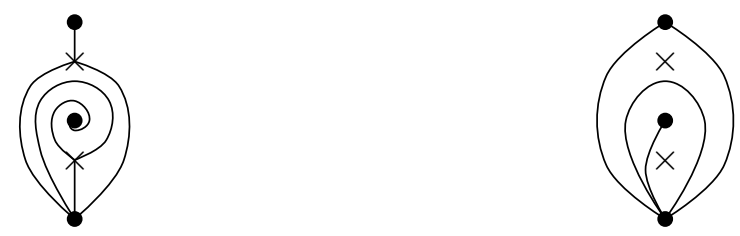

Figure 31. Degenerate horizontal strip (left) and inner arc (right)

Note that the arcs corresponding to the degenerate horizontal strips are the inner $\operatorname{arcs}$ in $T$. See Figure 31,

Remark 6.10. For each Stokes region $D$ which is a half plane, we can naturally identify a representative $\beta^{\prime}$ of trajectories in $D$ with the edge $\delta$ connecting the two marked points at the boundary component for $p_{i}$ corresponding to the tangent directions of the both ends of $\beta^{\prime}$ as in Figure 30 .

Lemma 6.11. Every triangle of $T$ contains exactly one midpoint.

Proof. The claim is divided into the following two claims.

- Every triangle in $T$ contains at most one zero.

- Every triangle in $T$ contains at least one zero.

Both claims follow from the classification of Stokes regions in Figure 29 and the construction of the triangulation $T$.

In summary, we have the desired extension of Proposition 6.9.

Proposition 6.12. The n-tuple $T$ is a labeled Stokes triangulation of $(\mathbf{S}, \mathbf{M}, \mathbf{A})$.

We call $T=T(\phi)$ a labeled Stokes triangulation of $(\mathbf{S}(\phi), \mathbf{M}(\phi), \mathbf{A}(\phi))$ associated with $G=G(\phi)$.

Remark 6.13. Assumption 2.3 for $\phi$ automatically guarantees Assumption 5.1 for the corresponding bordered surface $(\mathbf{S}(\phi), \mathbf{M}(\phi))$, except for the following cases:

- a once punctured monogon,

- an unpunctured triangle. 


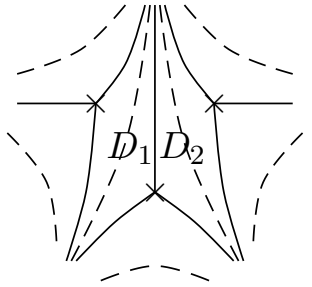

(a)

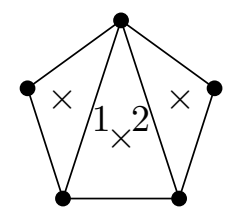

(b)

Figure 32. Example of labeled Stokes graph (drawn schematically) and associated labeled Stokes triangulation.

These exceptional cases are trivial from the cluster algebraic point of view, and we do not mind this discrepancy seriously. (However, they are basic and important examples in the exact WKB analysis.)

Example 6.14 (Pentagon relation (3)). Let us take $\Sigma=\mathbb{P}^{1}$ and the quadratic differential $\phi=Q(z) d z$ with $Q_{0}(z)=z(z+1)(z+i)$ in Figure 3 (b). The quadratic differential $\phi$ has zeros at $-i, 0$, and -1 . It has also a pole $p_{1}=\infty$ with order 7 . Thus, there are five tangent directions at $p$. The labeled Stokes graph of $\phi$ in $\mathbb{C}$ is drawn schematically (i.e., up to isotopy and rotation) in Figure 32 (a). Then, we have the associated labeled Stokes triangulation as in Figure 32 (b), where the boundary of the pentagon is identified with the boundary of the hole cut out around the pole $p=\infty$.

\subsection{Signed flips and signed pops of Stokes graphs}

In Section 3 we already treated the flips and the pops of Stokes graphs. Here, we refine them as the signed flips and the signed pops to incorporate them with the cluster algebra formulation.

To start, let us formulate the mutation of Stokes graphs in a more general situation than before. Suppose that there is a continuous 1-parameter family of quadratic differentials $\left\{\phi_{t} \mid 0 \leq t \leq 1\right\}$ on $\Sigma$ satisfying the following conditions:

Condition 6.15. (i). The positions of zeros and poles of $\phi_{t}$ may change, but their orders remain the same through the deformation.

(ii). The Stokes graph $G_{t}=G\left(\phi_{t}\right)$ is saddle-free for any $0 \leq t \leq 1$.

By condition (i), there is a homeomorphism $\tau=\tau\left(\phi_{0}, \phi_{1}\right)$ of $\Sigma$, by which one can naturally identify the zeros and the poles of $G_{1}$ with those of $G_{0}$. Furthermore, by condition (ii) and [BS13, Proposition 4.9], $G_{1}$ is isotopic to $G_{0}$, namely, $G_{t}$ deforms continuously without changing its topology form $t=0$ to 1 . Thus, one can identify the Stokes regions of $G_{1}$ with those of $G_{0}$ by $\tau$. In particular, a given label of $G_{0}$ induces a label of $G_{1}$. We call this labeled Stokes graph $G_{1}$ a regular deformation of a labeled Stokes graph $G_{0}$.

Remark 6.16. The homeomorphism $\tau=\tau\left(\phi_{0}, \phi_{1}\right)$ also induces the pull-back $\tau^{*}(T)$ of any labeled Stokes triangulation $T$ of $\left(\mathbf{S}\left(\phi_{1}\right), \mathbf{M}\left(\phi_{1}\right), \mathbf{A}\left(\phi_{1}\right)\right)$ to a labeled Stokes 
triangulation of $\left(\mathbf{S}\left(\phi_{0}\right), \mathbf{M}\left(\phi_{0}\right), \mathbf{A}\left(\phi_{0}\right)\right)$. Let $T_{0}=T\left(\phi_{0}\right)$ and $T_{1}=T\left(\phi_{1}\right)$ be the labeled Stokes triangulations associated with $G_{0}=G\left(\phi_{0}\right)$ and $G_{1}=G\left(\phi_{1}\right)$, respectively. Then, by construction, $\tau^{*}\left(T_{1}\right)$ coincides with $T_{0}$.

On the other hand, when the saddle-free condition (ii) is violated at some $t=t_{0}$, the Stokes graph $G_{t}$ changes its topology at $t_{0}$. We call this phenomenon the mutation of Stokes graphs. In this paper, we concentrate on the simplest situation where such $G_{t_{0}}$ has a unique saddle trajectory under Assumption 2.14. Then, by [BS13, Proposition 5.5], the mutation of Stokes graphs locally reduces to the saddle reduction of the saddle trajectory of $G_{t_{0}}$ studied in Section 3.3. Thus, it is described by a flip and a pop, depending on whether the saddle trajectory is regular or degenerate.

Remark 6.17. When the Stokes graph $G_{t_{0}}$ have two saddle trajectories, besides the combinations of flips and pops, another type of mutation called a juggle may occur [GMN13. This is also an important subject, but we do not treat it in this paper.

As mentioned, we refine the flips and the pops as the signed flips and the signed pops in parallel with Stokes triangulations.

First, let us consider the signed flips. Suppose that a Stokes graph $G_{0}=G(\phi)$ of a quadratic differential $\phi$ has the unique saddle trajectory, which is regular. For a sufficiently small $\delta>0$, let $G_{ \pm \delta}=G\left(e^{ \pm 2 i \delta} \phi\right)$ be the saddle reductions of $G_{0}$ in Section 3.3. See Figure 33. We assign the same label to the pair of the Stokes regions of $G_{+\delta}$ and $G_{-\delta}$ which degenerate into the saddle trajectory of $G_{0}$. We also assign the same label to each pair of the Stokes regions of $G_{+\delta}$ and $G_{-\delta}$ which are naturally identified by an isotopy. Let $k$ be the label of the Stokes regions of $G_{+\delta}$ and $G_{-\delta}$ which degenerate to saddle trajectory. Then, we write $G_{-\delta}=\mu_{k}^{(+)}\left(G_{+\delta}\right)$ and $G_{+\delta}=\mu_{k}^{(-)}\left(G_{-\delta}\right)$ as labeled Stokes graphs.

Definition 6.18. For a pair of labeled Stokes graphs $G=G(\phi)$ and $G^{\prime}=G\left(\phi^{\prime}\right)$, suppose that there is a pair of labeled Stokes graphs $G_{+\delta}$ and $G_{-\delta}$ which are the saddle reductions of a Stokes graph $G_{0}$ with a unique regular saddle trajectory such that

- $G_{+\delta}$ and $G_{-\delta}$ are regular deformations of $G$ and $G^{\prime}$, respectively,

- $G_{-\delta}=\mu_{k}^{(+)}\left(G_{+\delta}\right)$ in the above sense.

Then, we write $G^{\prime}=\mu_{k}^{(+)}(G)$ and $G=\mu_{k}^{(-)}\left(G^{\prime}\right)$, and call $G^{\prime}$ a signed flip of $G$ at $k$ with sign + and vice versa.

Remark 6.19. It follows from [BS13, Proposition 4.9] that if $G^{\prime}=\mu_{k}^{(\varepsilon)}(G)$ and $G^{\prime \prime}=\mu_{k}^{(\varepsilon)}(G)$, then $G^{\prime \prime}$ is a regular deformation of $G^{\prime \prime}$. Namely, a signed flip $\mu_{k}^{(\varepsilon)}(G)$ is unique up to a regular deformation.

As expected, the signed flips of labeled Stokes graphs and labeled Stokes triangulations are compatible.

Proposition 6.20. Suppose that $G^{\prime}=\mu_{k}^{(\varepsilon)}(G)$. Let $T$ and $T^{\prime}$ be labeled Stokes triangulations associated with $G$ and $G^{\prime}$, respectively, and let $\tau=\tau\left(\phi, \phi^{\prime}\right)$ be a homeomorphism of $\Sigma$ naturally identifying the zeros and poles of $G$ and $G^{\prime}$. Then, $\mu_{k}^{(\varepsilon)}(T)=\tau^{*}\left(T^{\prime}\right)$ holds, where $\tau^{*}\left(T^{\prime}\right)$ is the pullback of $T^{\prime}$ induced by $\tau$. 


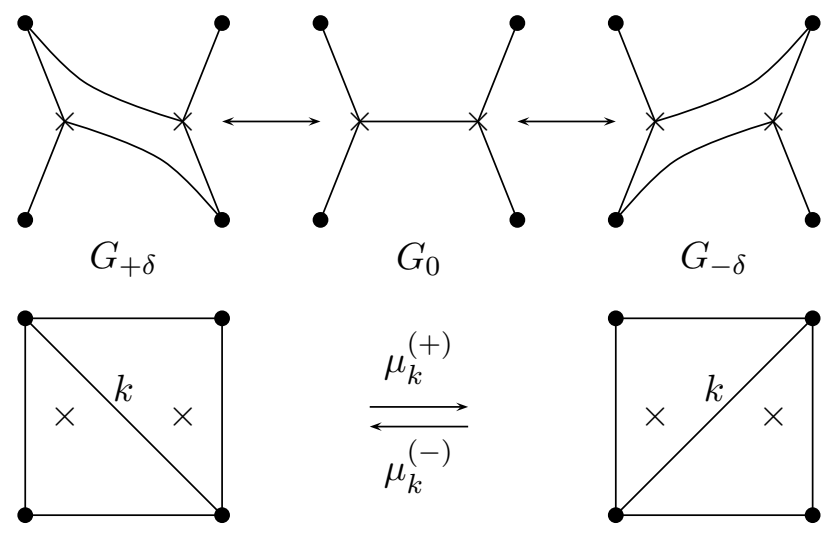

Figure 33. Signed flips of Stokes graphs (upper row) and Stokes triangulations (lower row).

Proof. Thanks to Remark 6.16, we can assume that $G=G_{\delta}$ and $G^{\prime}=G_{-\delta}$. Since the zeros and poles do not move for $G_{\theta}(-\delta \leq \theta \leq \delta), \tau$ is taken to be the identity map. Then, this is clear from Figure 33 .

Next, let us consider the signed pops. Let $G$ be a saddle-free labeled Stokes graph, $T$ be a labeled Stokes triangulation associated with $G$, and $p$ be a puncture inside a self-folded triangle in $T$. Then, a signed pop $G^{\prime}=\kappa_{p}^{(\varepsilon)}(G)$ at $p(\varepsilon= \pm)$ is defined in a parallel way by replacing a regular saddle trajectory of $G_{0}$ in the above with a degenerate one surrounding the double pole of $G_{0}$ corresponding to $p$. See Figure 34. The only speciality is that the labels of the inner and outer Stokes regions surrounding the double pole should be interchanged by the signed pops. The rest is the same as the signed flip and we do not repeat it. Again, we have the following compatibility.

Proposition 6.21. Suppose that $G^{\prime}=\kappa_{p}^{(\varepsilon)}(G)$. Let $T$ and $T^{\prime}$ be labeled Stokes triangulations associated with $G$ and $G^{\prime}$, respectively, and let $\tau=\tau\left(\phi, \phi^{\prime}\right)$ be a homeomorphism of $\Sigma$ naturally identifying the zeros and poles of $G$ and $G^{\prime}$. Then, $\kappa_{p}^{(\varepsilon)}(T)=\tau^{*}\left(T^{\prime}\right)$ holds, where $\tau^{*}\left(T^{\prime}\right)$ is the pullback of $T^{\prime}$ induced by $\tau$.

\subsection{Simple paths and simple cycles}

Let $\phi$ be a quadratic differential on $\Sigma$. Recall that we call elements of $H_{1}\left(\hat{\Sigma} \backslash \hat{P}_{0}, \hat{P}_{\infty}\right)$ and $H_{1}(\hat{\Sigma} \backslash \hat{P})$ paths and cycles, respectively, in Section 3 .

Let $\beta^{*}$ (resp., $\gamma^{*}$ ) be the image of a path $\beta$ (resp., a cycle $\gamma$ ) by the covering involution $\tau$ while keeping the direction. Let

$$
\begin{aligned}
\operatorname{Sym}\left(H_{1}\left(\hat{\Sigma} \backslash \hat{P}_{0}, \hat{P}_{\infty}\right)\right) & =\left\{\beta \in H_{1}\left(\hat{\Sigma} \backslash \hat{P}_{0}, \hat{P}_{\infty}\right) \mid \beta^{*}=\beta\right\}, \\
\operatorname{Sym}\left(H_{1}(\hat{\Sigma} \backslash \hat{P})\right) & =\left\{\gamma \in H_{1}(\hat{\Sigma} \backslash \hat{P}) \mid \gamma^{*}=\gamma\right\} .
\end{aligned}
$$

We introduce the $*$-equivalence of paths $\beta \equiv \beta^{\prime}$ (resp., cycles $\gamma \equiv \gamma^{\prime}$ ) by the condition $\beta-\beta^{\prime} \in \operatorname{Sym}\left(H_{1}\left(\hat{\Sigma} \backslash \hat{P}_{0}, \hat{P}_{\infty}\right)\right)\left(\right.$ resp., $\gamma-\gamma^{\prime} \in \operatorname{Sym}\left(H_{1}(\hat{\Sigma} \backslash \hat{P})\right)$. For example, $\beta \equiv-\beta^{*}$ and $\gamma \equiv-\gamma^{*}$ hold. 


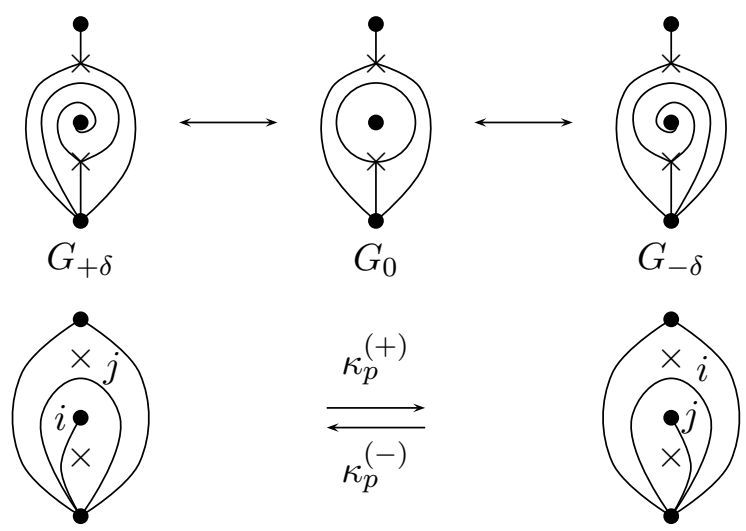

Figure 34. Signed pops of Stokes graphs (upper row) and Stokes triangulations (lower row).

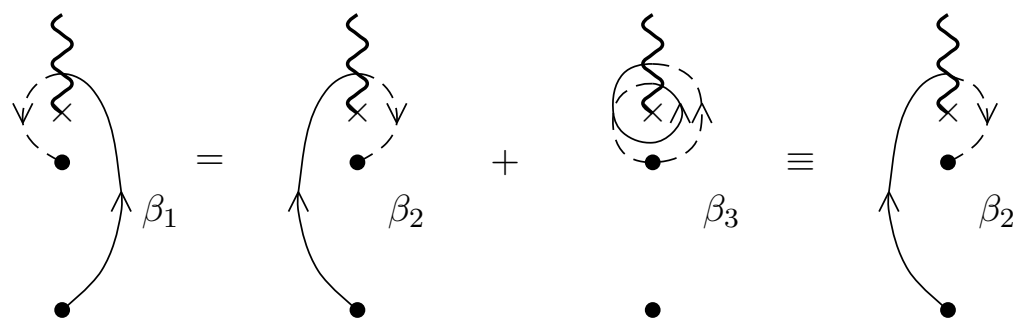

Figure 35. Typical deformations of paths modulo the $*$-equivalence.

Example 6.22. Typical deformations of paths and cycles modulo the $*$-equivalence are given in Figures 35 and 36. Observe that these deformations are not quite obvious (like puzzle rings!).

We introduce the quotients by the *-equivalence, namely,

$$
\begin{aligned}
\tilde{\Gamma}^{\vee} & =H_{1}\left(\hat{\Sigma} \backslash \hat{P}_{0}, \hat{P}_{\infty}\right) / \operatorname{Sym}\left(H_{1}\left(\hat{\Sigma} \backslash \hat{P}_{0}, \hat{P}_{\infty}\right)\right), \\
\tilde{\Gamma} & =H_{1}(\hat{\Sigma} \backslash \hat{P}) / \operatorname{Sym}\left(H_{1}(\hat{\Sigma} \backslash \hat{P})\right) .
\end{aligned}
$$

From now on, we identify paths $\beta$ and cycles $\gamma$ modulo the *-equivalence. In other words, we consider classes $[\beta] \in \tilde{\Gamma}^{\vee}$ and $[\gamma] \in \tilde{\Gamma}$ and denote them by their representatives $\beta$ and $\gamma$, for the notational simplicity. Any path $\beta \in \tilde{\Gamma}^{\vee}$ and any cycle $\gamma \in \tilde{\Gamma}$ are specified by presenting their representatives in a particular choice of branch. In Figure 37 some examples of representatives of a path $\beta \in \tilde{\Gamma}^{\vee}$ and a cycle $\gamma \in \tilde{\Gamma}$ in different branches are given.

Now we introduce one of key objects in our work.

Definition 6.23. Let $\phi$ be a saddle-free quadratic differential on $\Sigma$. Let $G=G(\phi)$ be a labeled Stokes graph of $\phi$, and let $D_{1}, \ldots, D_{n}$ be the Stokes regions of $G$ which are regular or degenerate horizontal strips. To each region $D_{i}$, we assign a path $\beta_{i} \in \tilde{\Gamma}^{\vee}$ and a cycle $\gamma_{i} \in \tilde{\Gamma}^{\vee}$ as follows: 

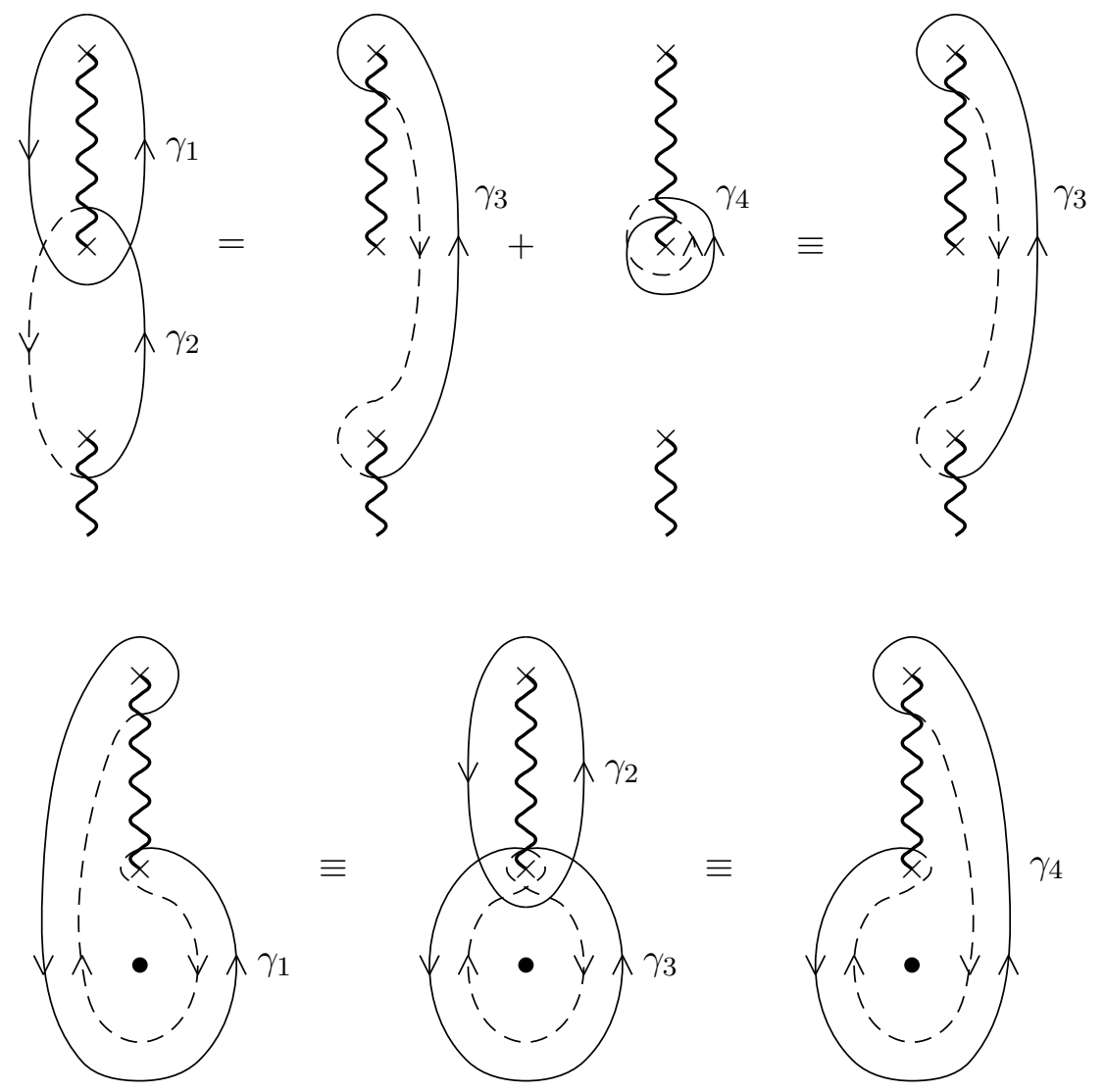

Figure 36. Typical deformations of cycles modulo the $*$-equivalence.
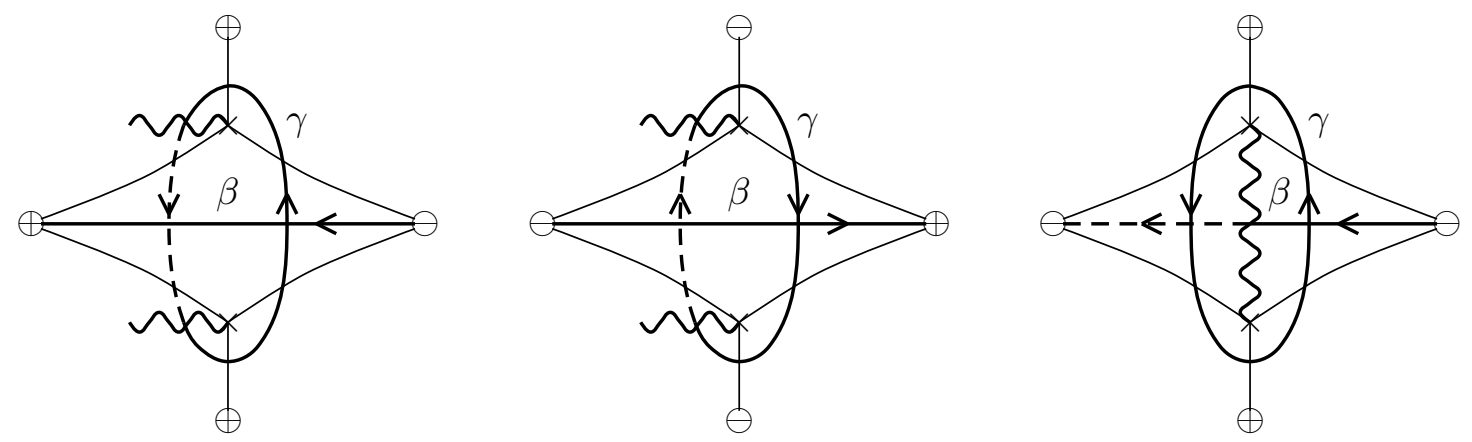

Figure 37. Examples of representatives of a path $\beta \in \tilde{\Gamma}^{\vee}$ and a cycle $\gamma \in \tilde{\Gamma}$ in different branches.

- If $D_{i}$ is a regular horizontal strip not surrounding a degenerate horizontal strip, $\beta_{i}$ and $\gamma_{i}$ are given in Figure 38. (The presented therein are their representatives in a particular choice of branch.)

- If $D_{i}$ is a degenerate horizontal strip and $D_{j}$ is the regular horizontal strip surrounding $D_{i}$, then $\beta_{i}, \beta_{j}, \gamma_{i}, \gamma_{j}$ are given in Figure 39. (Both figures in Figure 39 yield the same $*$-equivalence classes as demonstrated in Figures 35 and 36.) 


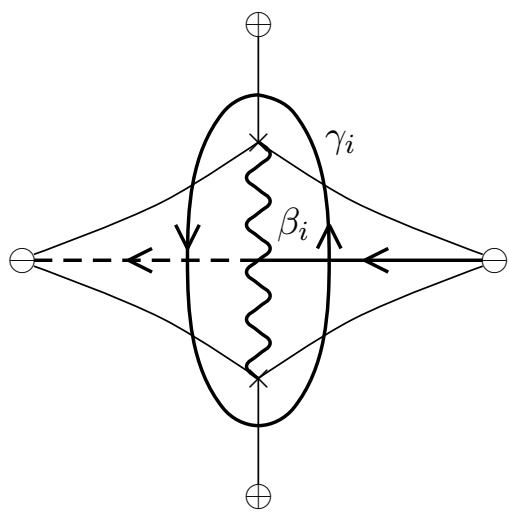

Figure 38. Simple path and simple cycle for a regular horizontal strip $D_{i}$ not surrounding a degenerate horizontal strip.
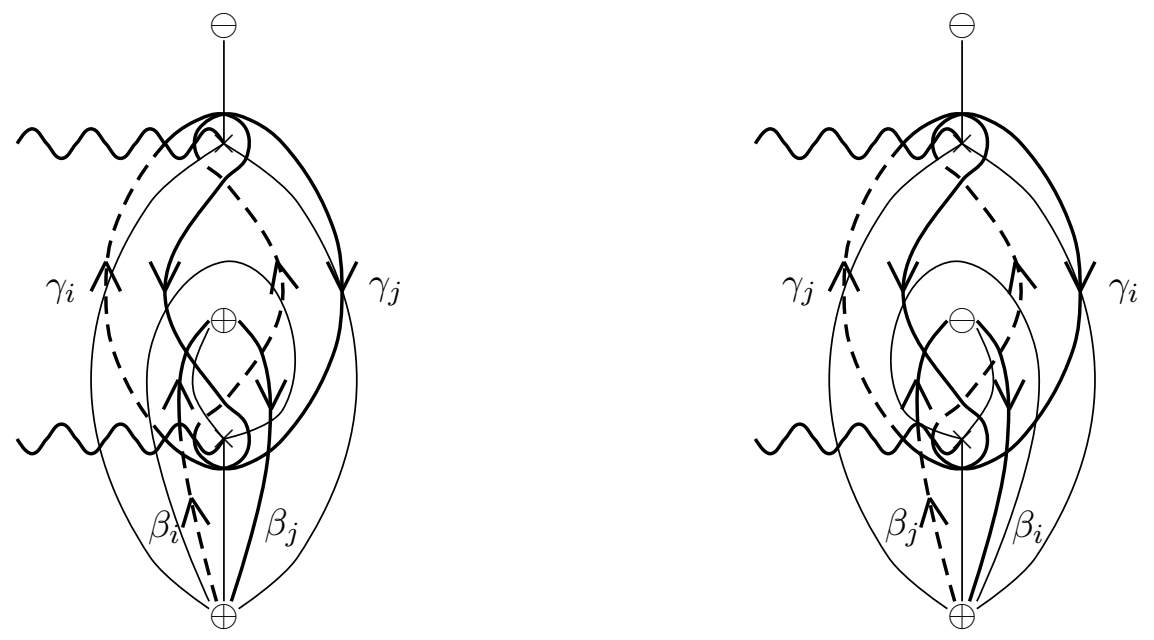

Figure 39. Simple paths and simple cycles for a degenerate horizontal strip $D_{i}$ and for the regular horizontal strip $D_{j}$ surrounding $D_{i}$.

We call $\beta_{1}, \ldots, \beta_{n} \in \Gamma^{\vee}$ and $\gamma_{1}, \ldots, \gamma_{n} \in \Gamma$ the simple paths and the simple cycles of $G$, respectively.

Remark 6.24. The simple cycles correspond to the modified basis in [BS13] in their convention of the homology group.

We define $\Gamma^{\vee}$ to be the subgroup of $\tilde{\Gamma}^{\vee}$ generated by $\beta_{1}, \ldots, \beta_{n}$. Similarly, we define $\Gamma$ to be the subgroup of $\tilde{\Gamma}$ generated by $\gamma_{1}, \ldots, \gamma_{n}$. It can be easily checked that the intersection forms in (3.1) and (3.2) induce the intersection forms $\langle\rangle:, \Gamma \times \Gamma^{\vee} \rightarrow \mathbb{Z}$ and $():, \Gamma \times \Gamma \rightarrow \mathbb{Z}$.

Proposition 6.25. We have

$$
\left\langle\gamma_{i}, \beta_{j}\right\rangle=\delta_{i j}
$$

so that the simple paths $\beta_{1}, \ldots, \beta_{n} \in \Gamma^{\vee}$ and the simple cycles $\gamma_{1}, \ldots, \gamma_{n} \in \Gamma$ are the dual bases to each other. 


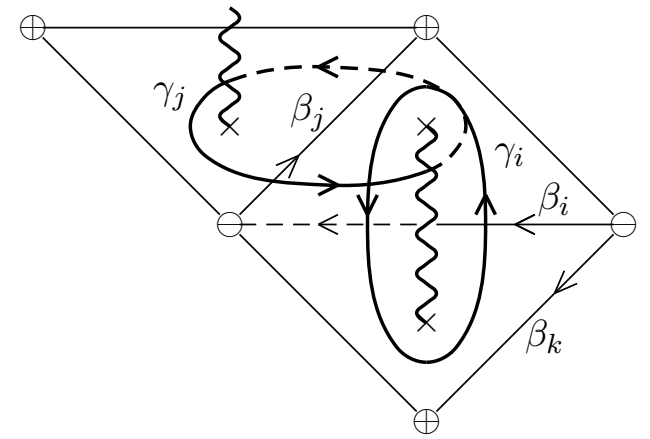

Figure 40. Calculation of the intersection number $\left(\gamma_{i}, \gamma_{j}\right)=1$.

Proof. This can be checked by inspecting Figures 38 and 39,

Let us observe that the simple paths and the simple cycles are naturally integrated into cluster algebra theory. Let $T$ be the labeled Stokes triangulation associated with $G$. The following formula is the first justification of the definition of the simple cycles.

Proposition 6.26. Let $B=\left(b_{i j}\right)_{i, j=1}^{n}$ be the adjacency matrix of $T$. Then, we have

$$
\left(\gamma_{i}, \gamma_{j}\right)=b_{i j}
$$

Proof. We prove it by case-check. There are essentially two cases to consider.

Case 1. Suppose that the regions $D_{i}$ and $D_{j}$ are regular horizontal strips. Then, $b_{i j}=1$ or 2 . The case $b_{i j}=1$ is depicted in Figure 40, and we see that $\left(\gamma_{i}, \gamma_{j}\right)=1=b_{i j}$. In the case $b_{i j}=2$, identify the paths $\beta_{j}$ and $-\beta_{k}$ in Figure 40, Then, we have $\left(\gamma_{i}, \gamma_{j}\right)=2=b_{i j}$.

Case 2. Suppose that the region $D_{i}$ is a degenerate horizontal strip and the region $D_{j}$ is the regular horizontal strip surrounding $D_{i}$. Then, by Figure 39 we have $\left(\gamma_{i}, \gamma_{j}\right)=0=b_{i j}$, while $\left(\gamma_{i}, \gamma_{k}\right)=\left(\gamma_{j}, \gamma_{k}\right)=b_{i k}=b_{j k}$ for any $k \neq i, j$.

Proposition 6.27. As an element of $\Gamma^{\vee}, \gamma_{i}$ decomposes as follows:

$$
\gamma_{i}=\sum_{j=1}^{n} b_{j i} \beta_{j}
$$

Proof. This can be verified by case-check using Figures 39 and 40 .

\subsection{Mutation of simple paths and simple cycles}

Let us examine how the simple paths and the simple cycles transform (= mutate) under the mutation of Stokes graphs. Suppose that there are two labeled Stokes graphs $G=G(\phi)$ and $G^{\prime}=G\left(\phi^{\prime}\right)$ which are related by a signed flip or a signed pop. Let us distinguish the corresponding homology groups $\Gamma^{\vee}$ and $\Gamma$ for $\phi$ and $\phi^{\prime}$ as $\Gamma_{G}^{\vee}, \Gamma_{G}$ and 


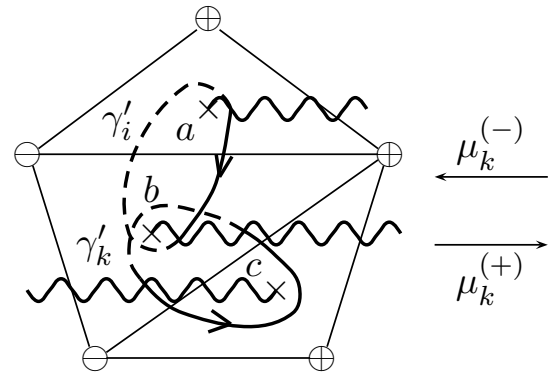

$G^{\prime}$

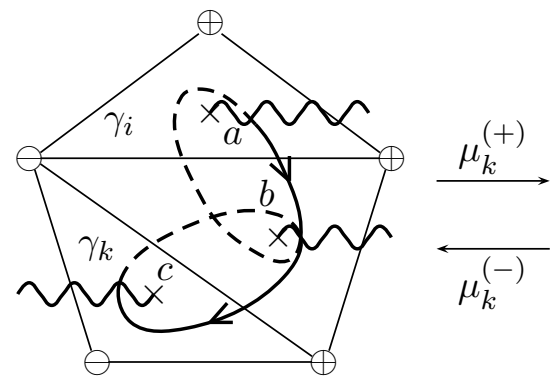

$G$

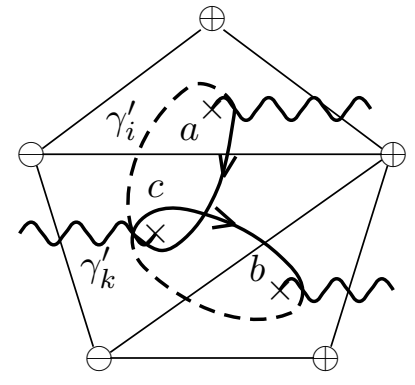

$G^{\prime}$

Figure 41. Mutation of simple cycles for signed flips.

$\Gamma_{G^{\prime}}^{\vee}, \Gamma_{G^{\prime}}$, respectively. By assumption, the zeros and poles of $\phi$ continuously move to those of $\phi^{\prime}$. This induces the canonical isomorphisms of the homology groups

$$
\tau_{G, G^{\prime}}^{\vee}: \Gamma_{G^{\prime}}^{\vee} \stackrel{\sim}{\rightarrow} \Gamma_{G}^{\vee}, \quad \tau_{G, G^{\prime}}: \Gamma_{G^{\prime}} \stackrel{\sim}{\rightarrow} \Gamma_{G}
$$

Let $\beta_{1}, \ldots, \beta_{n} \in \Gamma_{G}^{\vee}$ and $\gamma_{1}, \ldots, \gamma_{n} \in \Gamma_{G}$ be the simple paths and cycles of $G$, and $\beta_{1}^{\prime}, \ldots, \beta_{n}^{\prime} \in \Gamma_{G^{\prime}}^{\vee}$ and $\gamma_{1}^{\prime}, \ldots, \gamma_{n}^{\prime} \in \Gamma_{G^{\prime}}$ be the ones of $G^{\prime}$. Then, the isomorphisms $\tau_{G, G^{\prime}}^{\vee}$ and $\tau_{G, G^{\prime}}$ are explicitly given as follows.

Proposition 6.28. (a). (cf. [BS13, Lemma 9.11]) If $G$ and $G^{\prime}$ are related by the signed flip $G^{\prime}=\mu_{k}^{(\varepsilon)}(G)$, then the isomorphisms $\tau_{G, G^{\prime}}^{\vee}$ and $\tau_{G, G^{\prime}}$ are given by

$$
\begin{aligned}
& \tau_{G, G^{\prime}}^{\vee}\left(\beta_{i}^{\prime}\right)= \begin{cases}-\beta_{k}+\sum_{j=1}^{n}\left[-\varepsilon b_{j k}\right]_{+} \beta_{j} & i=k \\
\beta_{i} & i \neq k,\end{cases} \\
& \tau_{G, G^{\prime}}\left(\gamma_{i}^{\prime}\right)= \begin{cases}-\gamma_{k} & i=k \\
\gamma_{i}+\left[\varepsilon b_{k i}\right]_{+} \gamma_{k} & i \neq k .\end{cases}
\end{aligned}
$$

(b). If $G$ and $G^{\prime}$ are related by the signed pop $G^{\prime}=\kappa_{p}^{(\varepsilon)}(G)$, then the isomorphisms $\tau_{G, G^{\prime}}^{\vee}$ and $\tau_{G, G^{\prime}}$ are trivial, i.e.,

$$
\tau_{G, G^{\prime}}^{\vee}\left(\beta_{i}^{\prime}\right)=\beta_{i}, \quad \tau_{G, G^{\prime}}\left(\gamma_{i}^{\prime}\right)=\gamma_{i}
$$

Proof. (a). Two transformations (6.9) and (6.10) preserve the duality (6.5) (see [Nak12, Section 3.3]). Therefore, it is enough to prove (6.9). This can be done by case-check with respect to the configuration of $\gamma_{i}$ and $\gamma_{k}$. We only present the most typical case in Figure 41, where $b_{k i}=1$. Then, for $\varepsilon=+, \gamma_{i}^{\prime}=\gamma_{i}+\gamma_{k}$ by the deformation of cycles in Figure 36, for $\varepsilon=-, \gamma_{i}^{\prime}=\gamma_{i}$, and in either case, $\gamma_{k}^{\prime}=-\gamma_{k}$. This agrees with (6.10).

(b). Again, it is enough to prove it for the simple paths. The essential case is given in Figure 42, where the label of the arcs is the same one in Figure 34.

Proposition 6.28 tells that the simple paths and the simple cycles mutate as the (logarithm of) monomial $x$-variables and monomial $y$-variables in Section 4.6, respectively. The formula for the simple cycles already appeared in BBS13, Lemma 9.11] as the mutation of the modified basis therein. 


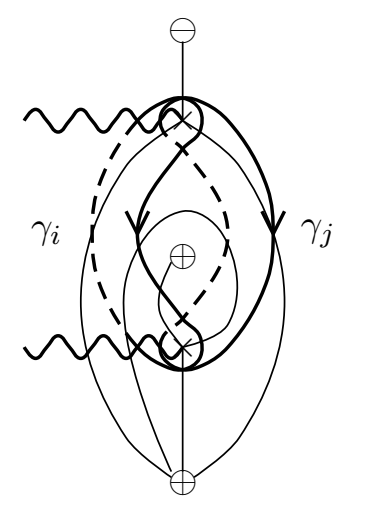

G

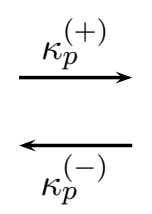

Figure 42. Mutation of simple cycles for signed pops.

\subsection{Periodicity of signed mutations and signed flips}

Let $G^{0}=G(\phi)$ be a labeled Stokes graph taken as the "initial" one, let $T^{0}$ be the associated labeled Stokes triangulation of $(\mathbf{S}(\phi), \mathbf{M}(\phi), \mathbf{A}(\phi))$, and let $B^{0}=B\left(T^{0}\right)$ be the adjacency matrix of $T^{0}$. For the cluster algebra $\mathcal{A}\left(B^{0}, x^{0}, y^{0} ; \mathbb{Q}_{+}\left(y^{0}\right)\right)$, suppose that $\vec{k}=\left(k_{1}, \ldots, k_{N}\right)$ is a $\nu$-period of the initial seed $\left(B^{0}, x^{0}, y^{0}\right)$; namely, we have a mutation sequence

$$
\begin{aligned}
(B(1), x(1), y(1))=\left(B^{0}, x^{0}, y^{0}\right) \stackrel{\mu_{k_{1}}}{\rightarrow}(B(2), x(2), y(2)) \stackrel{\mu_{k_{2}}}{\rightarrow} \cdots \\
\\
\quad \cdots \stackrel{\mu_{k_{N}}}{\rightarrow}(B(N+1), x(N+1), y(N+1))
\end{aligned}
$$

such that $y_{\nu(i)}(N+1)=y_{i}^{0}$. Let $\varepsilon_{t}=\varepsilon\left(y_{k_{t}}(t)\right)(t=1, \ldots, N)$ be the tropical sign of $y_{k_{t}}(t)$ with respect to the initial $y$-variables $y^{0}=y(1)$.

To make use of such a sequence in our application, we need to reexpress it by signed mutations and signed pops. For that purpose, we introduce a mutation sequence of labeled Stokes triangulations of $(\mathbf{S}(\phi), \mathbf{M}(\phi), \mathbf{A}(\phi))$,

$$
T(1)=T \stackrel{\stackrel{\tilde{\mu}_{k_{1}}^{\left(\varepsilon_{1}\right)}}{\rightarrow}}{\rightarrow} T(2) \stackrel{\tilde{\mu}_{k_{2}}^{\left(\varepsilon_{2}\right)}}{\rightarrow} \cdots \stackrel{\tilde{\mu}_{k_{N}}^{\left(\varepsilon_{N}\right)}}{\rightarrow} T(N+1) \stackrel{\tilde{\xi}}{\rightarrow} T(N+2) .
$$

Here, for $T(t)=\left(\alpha_{i}(t)\right)_{i=1}^{n}$, we set

$$
\tilde{\mu}_{k_{t}}^{\left(\varepsilon_{t}\right)}= \begin{cases}\mu_{k_{t}}^{\left(\varepsilon_{t}\right)} & \text { if } \alpha_{k_{t}}(t) \text { in } T(t) \text { is flippable } \\ \mu_{k_{t}}^{\left(\varepsilon_{t}\right)} \circ \kappa_{p_{t}}^{\left(s_{t}\right)} & \text { otherwise, }\end{cases}
$$

where $p_{t}$ is the puncture inside the self-folded triangle in $T(t)$ which $\alpha_{k_{t}}(t)$ belongs to, and the signs $s_{t} \in\{+,-\}$ are determined by the following rule:

- For $t=1, \ldots, N$, let

$$
n(t):=\left|\left\{t^{\prime} \mid 1 \leq t^{\prime}<t, p_{t^{\prime}}=p_{t}\right\}\right|,
$$

and we set $s_{t}=(-1)^{n(t)}$. Namely, if a puncture $p=p_{t}$ appears at the first time in the sequence (6.13) at $t$, then $s_{t}=+$, and the sign for $p$ alternates after that. 
Also, we set

$$
\tilde{\kappa}=\prod_{p}{ }^{\prime} \kappa_{p}^{(-)}
$$

where the product runs over the punctures $p$ such that $\left|\left\{1 \leq t \leq N \mid p_{t}=p\right\}\right|$, i.e., the number of the appearance of $p$ in the sequence (6.13), is odd.

Remark 6.29. If we project the sequence (6.13) to the sequence of labeled signed triangulations in Section 5.4 by forgetting the signs of flips and pops, the periodicity of the sequence (6.12) and Theorem 5.20 imply that the labeled signed triangulation at $t=N+1$, say, $T^{\prime}(N+1)$ is pop-equivalent to the initial one $T^{\prime}(1)$. Meanwhile, if $\left|\left\{1 \leq t \leq N \mid p_{t}=p\right\}\right|$ is odd for a puncture $p$, then the sign $\sigma_{p}$ at $p$ of $T^{\prime}(N+1)$ and $T^{\prime}(1)$ is opposite. These together mean that $p$ is inside the self-folded triangle in $T^{\prime}(N+1)$ so that it should be popped; therefore, it is also so in $T(N+1)$. This guarantees that the signed pops of $\tilde{\kappa}$ in (6.16) is well-defined.

First, let us consider a mutations sequence of labeled extended seeds which is parallel to (6.13),

$$
\begin{gathered}
(B(1), x(1), y(1), \tilde{y}(1))=\left(B^{0}, x^{0}, y^{0}, \tilde{y}^{0}\right) \stackrel{\tilde{\mu}_{k_{1}}^{\left(\varepsilon_{1}\right)}}{\rightarrow}(B(2), x(2), y(2), \tilde{y}(2)) \stackrel{\tilde{\mu}_{k_{2}}^{\left(\varepsilon_{2}\right)}}{\rightarrow} \cdots \\
\ldots \stackrel{\tilde{\mu}_{k_{N}}^{\left(\varepsilon_{N}\right)}}{\rightarrow}(B(N+1), \ldots, \tilde{y}(N+1)) \stackrel{\tilde{\kappa}}{\rightarrow}(B(N+2), \ldots, \tilde{y}(N+2)),
\end{gathered}
$$

where $\tilde{\mu}_{k_{t}}^{\left(\varepsilon_{t}\right)}$ and $\tilde{\kappa}$ are the ones in (6.14) and (6.16) but acting on extended seeds.

Proposition 6.30. The mutation sequence (6.17) of extended seeds is $\nu$-periodic in the following sense:

$$
\begin{gathered}
b_{\nu(i) \nu(j)}(N+2)=b_{i j}(1), \quad x_{\nu(i)}(N+2)=x_{i}(1), \\
y_{\nu(i)}(N+2)=y_{i}(1), \quad \tilde{y}_{i}(N+2)=\tilde{y}_{i}(1) .
\end{gathered}
$$

Proof. It is enough to show the periodicity of $\tilde{y}$ - and $x$-variables. First, let us show the periodicity of $\tilde{y}$-variables. By the definition of $\tilde{\kappa}$ in (6.16), for each puncture $p$, the numbers of $\kappa_{p}^{(+)}$and $\kappa_{p}^{(-)}$appearing in the sequence (6.17) are equal. Then, the periodicity of $x$-variables follows from Proposition 5.31 and the property $\kappa_{p}^{ \pm} \kappa_{p}^{\mp}=1$. It also implies $\tilde{y}_{p}(N+2)=\tilde{y}_{p}(1)$ by (5.5) .

Next, consider a mutation sequence of labeled Stokes graphs,

$$
G(1)=G \stackrel{\tilde{\mu}_{k_{1}}^{\left(\varepsilon_{1}\right)}}{\rightarrow} G(2) \stackrel{\tilde{\mu}_{k_{2}}^{\left(\varepsilon_{2}\right)}}{\rightarrow} \cdots \stackrel{\tilde{\mu}_{k_{N}}^{\left(\varepsilon_{N}\right)}}{\rightarrow} G(N+1) \stackrel{\tilde{\tilde{G}}}{\rightarrow} G(N+2),
$$

where $\tilde{\mu}_{k_{t}}^{\left(\varepsilon_{t}\right)}$ and $\tilde{\kappa}$ are the same ones in (6.14) and (6.16) but for labeled Stokes graphs.

Let $\beta_{i}(t)$ and $\gamma_{i}(t)$ be the simple paths and the simple cycles of $G(t)$. Let

$$
\tau_{G(t), G(t+1)}^{\vee}: \Gamma_{G(t+1)}^{\vee} \stackrel{\sim}{\rightarrow} \Gamma_{G(t)}^{\vee}, \quad \tau_{G(t), G(t+1)}: \Gamma_{G(t+1)} \stackrel{\sim}{\rightarrow} \Gamma_{G(t)}
$$


be the isomorphisms given by Proposition 6.28 for the sequence (6.19), and let

$$
\tau_{G(1), G(N+2)}^{\vee}: \Gamma_{G(N+2)}^{\vee} \stackrel{\sim}{\rightarrow} \Gamma_{G(1)}^{\vee}, \quad \tau_{G(1), G(N+2)}: \Gamma_{G(N+2)} \stackrel{\sim}{\rightarrow} \Gamma_{G(1)}
$$

be their compositions.

Then, we have the following $\nu$-periodicity of the simple paths and the simple cycles for the sequence (6.19).

Proposition 6.31.

$$
\begin{aligned}
& \tau_{G(1), G(N+2)}^{\vee}\left(\beta_{\nu(i)}(N+2)\right)=\beta_{i}(1), \\
& \tau_{G(1), G(N+2)}\left(\gamma_{\nu(i)}(N+2)\right)=\gamma_{i}(1) .
\end{aligned}
$$

Proof. Let us first show (6.23). By Proposition 6.28 and the choice of the signs $\varepsilon_{t}$, the simple cycles exactly transform as the logarithm of the tropical $y$-variables (see (4.18)) for the sequence (6.12). Therefore, we have the periodicity (6.23). The periodicity (6.22) follows from (6.23) and the duality in Proposition 6.25.

To conclude the section, let us remark that Proposition 6.30 and Conjecture 6.6 together imply the following $\nu$-periodicity of the labeled Stokes triangulations for the sequence (6.13).

Conjecture 6.32. As arcs in $(\mathbf{S}(\phi), \mathbf{M}(\phi), \mathbf{A}(\phi))$,

$$
\alpha_{\nu(i)}(N+2)=\alpha_{i}(1) .
$$

We have a partial result on the conjecture.

Proposition 6.33. The equality (6.24) is true for any $i$ such that both endpoints of $\alpha_{i}(1)$ are on the boundary of $\mathbf{S}(\phi)$. In particular, Conjecture 6.32 is true if $(\mathbf{S}(\phi), \mathbf{M}(\phi))$ have no punctures.

Proof. We have the following facts.

Fact 1. Let us temporarily forget the signs of all signed flips and pops in the sequence (6.13), and regard it as a sequence of signed ideal triangulations in Section 5.4. Then, by Theorem 5.20, we deduce that the equality (6.24) holds as arcs in $(\mathbf{S}(\phi), \mathbf{M}(\phi))$.

Fact 2. The group $\operatorname{Sym}\left(H_{1}\left(\hat{\Sigma} \backslash \hat{P}_{0}, \hat{P}_{\infty}\right)\right)$ in (6.1) is generated by two types of elements of $H_{1}\left(\hat{\Sigma} \backslash \hat{P}_{0}, \hat{P}_{\infty}\right)$; one is $\gamma+\gamma *$, where $\gamma$ is any path connecting two points in $\hat{P}_{\infty}$, and the other is any cycle (= closed path) in $\hat{\Sigma} \backslash \hat{P}_{0}$ around a point in $\hat{P}_{0}$.

Fact 3. By (6.22), the periodicity $\tau_{G(1), G(N+2)}^{\vee}\left(\beta_{\nu(i)}(N+2)\right)=\beta_{i}(1)$ holds.

Fact 4. By the definition of arcs, neither $\alpha_{\nu(i)}(N+2)$ nor $\alpha_{i}(1)$ intersects itself.

These facts, together with the assumption that both endpoints of $\alpha_{i}(1)$ are on the boundary of $\mathbf{S}(\phi)$, imply the equality (6.24) as arcs in $(\mathbf{S}(\phi), \mathbf{M}(\phi), \mathbf{A}(\phi))$. 


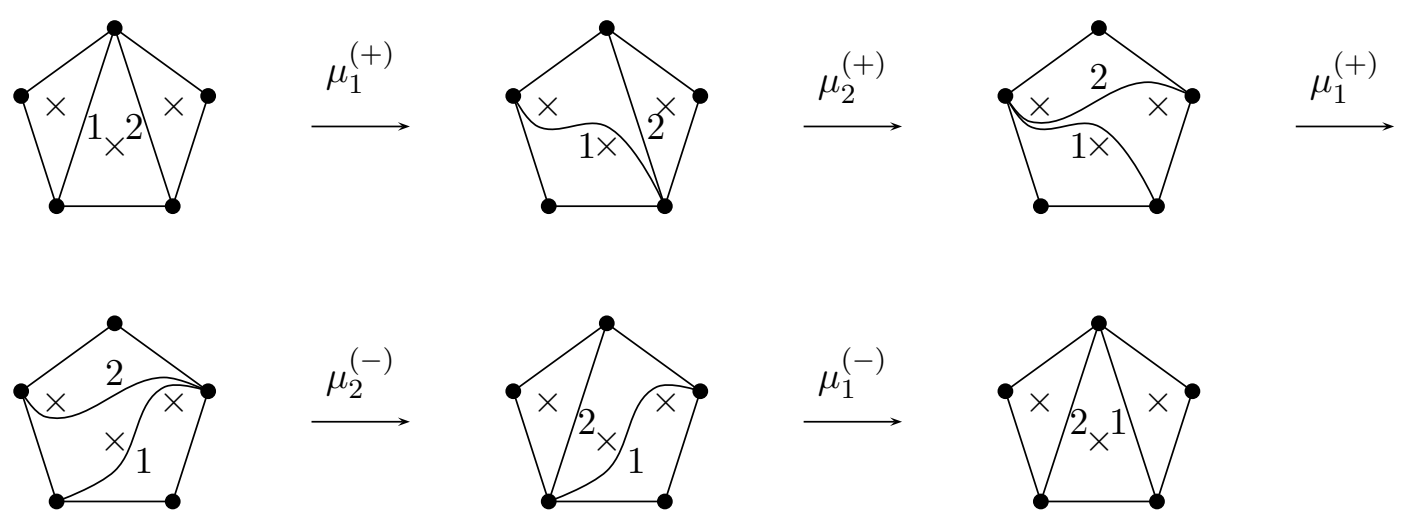

Figure 43. Pentagon relation of labeled Stokes triangulations.

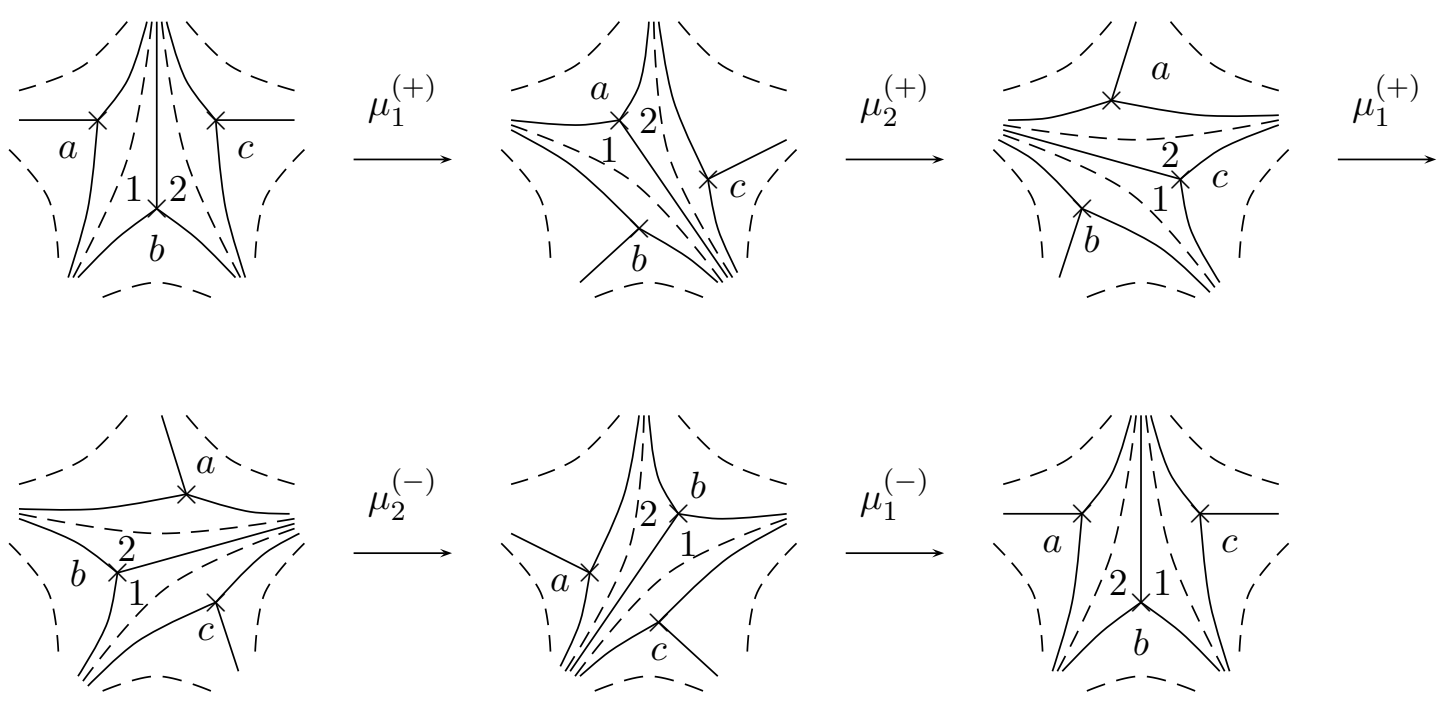

Figure 44. Pentagon relation of labeled Stokes graphs (drawn schematically).

Remark 6.34. On the other hand, if an $\operatorname{arc} \alpha_{i}$ has an endpoint at a puncture, one cannot deduce the equality (6.24) as above due to the deformation of paths in Figure 35. In the above proof, such a deformation does not occur because of the presence of the boundary at each endpoint.

Example 6.35 (Pentagon relation (4)). Let observe how the periodicity (6.24) occurs in our running Examples 4.10, 5.22, and 6.14. Let us take the labeled Stokes graph in Figure 32 (a) as the initial labeled Stokes graph. We apply the mutation sequence $\vec{k}=(1,2,1,2,1)$ in Examples 4.10 and 5.22 . According to (4.28), we take the sequence of the tropical signs $\vec{\varepsilon}=(+,+,+,-,-)$. Then, the pentagon relations of the corresponding labeled Stokes triangulations and labeled Stokes graphs are presented in Figures 43 and 44, respectively. Note that the boundary trajectories in Figure 44 vanish in $\Gamma$ and $\Gamma^{\vee}$.

Example 6.36. Let us illustrate the mutation sequence (6.13) involving signed pops. We consider the mutation sequence of labeled seeds with period 4 represented by the 


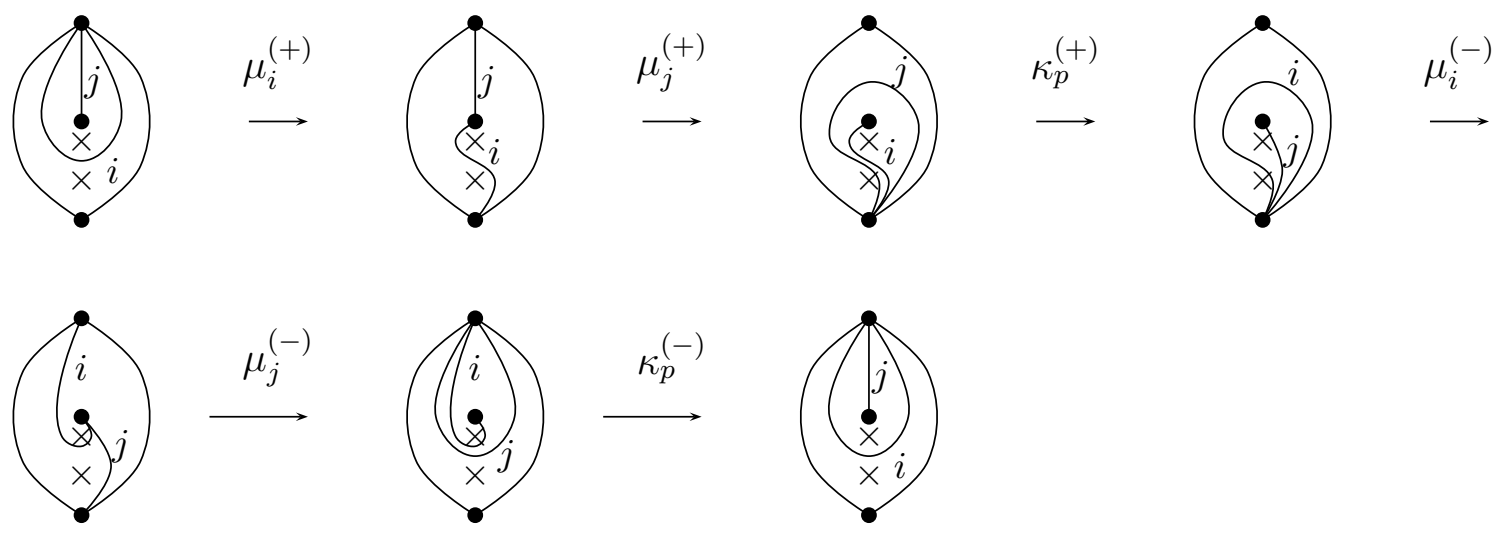

Figure 45. Example of periodicity of labeled Stokes triangulations involving pops.

labeled tagged triangulations in Figure 19, See also Figure 23. Then, the corresponding mutation sequence of the labeled Stokes triangulations is given by Figure 45, where

$$
\tilde{\mu}_{k_{1}}^{\left(\varepsilon_{1}\right)}=\mu_{i}^{(+)}, \quad \tilde{\mu}_{k_{2}}^{\left(\varepsilon_{2}\right)}=\mu_{j}^{(+)}, \quad \tilde{\mu}_{k_{3}}^{\left(\varepsilon_{3}\right)}=\mu_{i}^{(-)} \circ \kappa_{p}^{(+)}, \quad \tilde{\mu}_{k_{4}}^{\left(\varepsilon_{4}\right)}=\mu_{j}^{(-)}, \quad \kappa=\kappa_{p}^{(-)} .
$$

Thus, the desired periodicity (6.24) holds in this example.

\section{Mutation of Voros symbols}

Here we combine the analytic and geometric results in Sections 3 and 6 and show that the Voros symbols for the simple paths and the simple cycles mutate exactly as $x$-variables and $\hat{y}$-variables in our extended seeds.

\subsection{Mutation formula of Voros symbols for signed flips}

Let us return to the situation in Section 3.6. Let $Q(z, \eta)$ be the potential of a Schrödinger equation (2.1), and let $\phi=Q_{0}(z) d z^{\otimes 2}$ be the associated quadratic differential. Assume that the Stokes graph $G_{0}=G(\phi)$ has a unique regular saddle trajectory $\ell_{0}$. Let $Q^{(\theta)}(z, \eta)$ be the $S^{1}$-family for $Q(z, \eta)$ in (3.18). We choose a sufficiently small $\delta>0$ such that $G_{+\delta}=G\left(\phi_{+\delta}\right)$ and $G_{-\delta}=G\left(\phi_{-\delta}\right)$ are the saddle reductions of $G_{0}$. Let us fix a sign $\varepsilon= \pm$. Then, we set $G=G_{\varepsilon \delta}$ and $G^{\prime}=G_{-\varepsilon \delta}$. We assume that $G$ and $G^{\prime}$ are labeled so that they are related by the signed flip $G^{\prime}=\mu_{k}^{(\varepsilon)}(G)$. See Figure 33.

Let $\beta_{1}, \ldots \beta_{n} \in \Gamma_{G}^{\vee}$ and $\gamma_{1}, \ldots, \gamma_{n} \in \Gamma_{G}$ be the simple paths and the simple cycles of $G$ introduced in Section 6.4. We denote the Voros symbols for the potential $Q^{(\varepsilon \delta)}(z, \eta)$ with respect to them by

$$
e^{W_{i}}=e^{W_{\beta_{i}}^{(\varepsilon \delta)}}, \quad e^{V_{i}}=e^{V_{\gamma_{i}}^{(\varepsilon \delta)}},
$$

where we use the notation in (3.21). We also introduce

$$
e^{v_{i}}=e^{\eta v_{\gamma_{i}}^{(\varepsilon \delta)}}, \quad v_{\gamma}^{(\theta)}=\int_{\gamma} \sqrt{Q_{0}^{(\theta)}(z)} d z=e^{i \theta} \int_{\gamma} \sqrt{Q_{0}(z)} d z .
$$



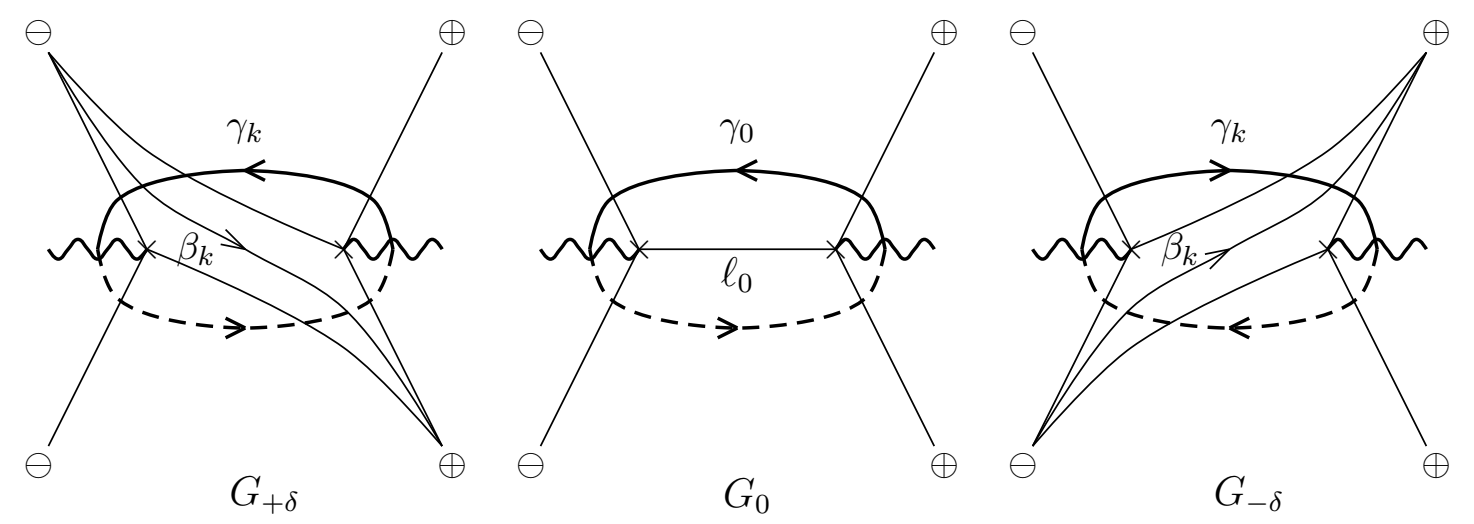

Figure 46. Cycles $\gamma_{0}$ and $\gamma_{k}$.

Thus, $e^{v_{i}}$ is the exponential factor of $e^{V_{i}}$.

Let $T$ be a labeled Stokes triangulation associated with $G$, and let $B$ be the adjacency matrices of $T$.

Lemma 7.1. The following relation holds.

$$
e^{V_{i}}=e^{v_{i}} \prod_{j=1}^{n}\left(e^{W_{j}}\right)^{b_{j i}}
$$

Proof. We have

$$
V_{i}=\oint_{\gamma_{i}} S_{\text {odd }}^{(\varepsilon \delta)}(z, \eta) d z=\oint_{\gamma_{i}}\left(\eta \sqrt{Q_{0}^{(\varepsilon \delta)}(z)}+S_{\text {odd }}^{\mathrm{reg}(\varepsilon \delta)}(z, \eta)\right) d z=v_{i}+\sum_{j=1}^{n} b_{j i} W_{j},
$$

where the last equality is due to Proposition 6.27.

Note that the relation (7.3) is parallel to the one for the $\hat{y}$-variables in (4.9).

Let $\gamma_{0} \in \Gamma_{G}$ be the saddle class associated with $\ell_{0}$ defined in Section 3.2 .

Lemma 7.2. The saddle class $\gamma_{0}$ coincides with $\varepsilon \gamma_{k}$.

Proof. In the case $\varepsilon=+$, where $G=G_{+\delta}, \gamma_{0}=\gamma_{k}$ holds as in Figure 46. Similarly, in the case $\varepsilon=-$, where $G=G_{-\delta}, \gamma_{k}=-\gamma_{0}$ holds as in Figure 46.

Using $\gamma_{k}$ instead of $\gamma_{0}$, the jump formula in Theorem 3.10 (a) is restated as follows.

Proposition 7.3. For any path $\beta \in \Gamma_{G}^{\vee}$ and any cycle $\gamma \in \Gamma_{G}$, we have

$$
\begin{aligned}
\lim _{\delta \rightarrow+0} \mathcal{S}\left[e^{W_{\beta}^{(-\varepsilon \delta)}}\right] & =\lim _{\delta \rightarrow+0} \mathcal{S}\left[e^{W_{\beta}^{(\varepsilon \delta)}}\left(1+\left(e^{V_{\gamma_{k}}^{(\varepsilon \delta)}}\right)^{\varepsilon}\right)^{-\left\langle\gamma_{k}, \beta\right\rangle}\right], \\
\lim _{\delta \rightarrow+0} \mathcal{S}\left[e^{V_{\gamma}^{(-\varepsilon \delta)}}\right] & =\lim _{\delta \rightarrow+0} \mathcal{S}\left[e^{V_{\gamma}^{(\varepsilon \delta)}}\left(1+\left(e^{V_{\gamma_{k}}^{(\varepsilon \delta)}}\right)^{\varepsilon}\right)^{-\left(\gamma_{k}, \gamma\right)}\right] .
\end{aligned}
$$


Proof. Let us show (7.4). When $\varepsilon=+, G=G_{+\delta}, G^{\prime}=G_{-\delta}$ and $\gamma_{0}=\gamma_{k}$ by Lemma 7.2 . Hence, we have the equality (7.4) immediately from (3.27). When $\varepsilon=-, G^{\prime}=G_{+\delta}$, $G=G_{-\delta}$ and $\gamma_{0}=-\gamma_{k}$ by Lemma 7.2 . Note that we have the equality

$$
\lim _{\delta \rightarrow+0} \mathcal{S}\left[e^{V_{\gamma_{0}}^{(-\delta)}}\right]=\lim _{\delta \rightarrow+0} \mathcal{S}\left[e^{V_{\gamma_{0}}^{(+\delta)}}\right]
$$

by (3.27) and $\left(\gamma_{0}, \gamma_{0}\right)=0$. Then, it follows from (3.27) that

$$
\begin{aligned}
\lim _{\delta \rightarrow+0} \mathcal{S}\left[e^{W_{\beta}^{(+\delta)}}\right] & =\lim _{\delta \rightarrow+0} \mathcal{S}\left[e^{W_{\beta}^{(-\delta)}}\left(1+e^{V_{\gamma_{0}}^{(+\delta)}}\right)^{\left(\gamma_{0}, \beta\right)}\right] \\
& =\lim _{\delta \rightarrow+0} \mathcal{S}\left[e^{W_{\beta}^{(-\delta)}}\left(1+e^{-V_{\gamma_{k}}^{(-\delta)}}\right)^{-\left(\gamma_{k}, \beta\right)}\right],
\end{aligned}
$$

which is the desired equality for $\varepsilon=-$. The equality (17.5) is proved in the same manner.

We emphasize the following "nonjump" property of the integral in (7.2).

Lemma 7.4. For any cycle $\gamma \in \Gamma_{G}$, we have

$$
\lim _{\delta \rightarrow+0} \mathcal{S}\left[e^{\eta v_{\gamma}^{(-\varepsilon \delta)}}\right]=\lim _{\delta \rightarrow+0} \mathcal{S}\left[e^{\eta v_{\gamma}^{(\varepsilon \delta)}}\right]
$$

Proof. By the definition of the Borel sum of an exponential factor in Definition 2.10, we have $\mathcal{S}\left[e^{\eta v_{\gamma}^{(\theta)}}\right]=e^{\eta v_{\gamma}^{(\theta)}}$. Thus, both hand sides of (7.8) equal to $e^{\eta v_{\gamma}^{(0)}}$.

Let $\beta_{1}^{\prime}, \ldots \beta_{n}^{\prime} \in \Gamma_{G^{\prime}}^{\vee}$ and $\gamma_{1}^{\prime}, \ldots, \gamma_{n}^{\prime} \in \Gamma_{G^{\prime}}$ be the simple paths and the simple cycles of $G^{\prime}$. Since the zeros and poles of $\phi_{\theta}$ are stable by the $S^{1}$-action, we have $\Gamma_{G}^{\vee}=\Gamma_{G^{\prime}}^{\vee}$ and $\Gamma_{G}=\Gamma_{G^{\prime}}$ with $\tau_{G, G^{\prime}}^{\vee}=\mathrm{id}$ and $\tau_{G, G^{\prime}}=\mathrm{id}$. Thus, (6.9) and (6.10) reduce to the direct equalities

$$
\begin{aligned}
& \beta_{i}^{\prime}= \begin{cases}-\beta_{k}+\sum_{j=1}^{n}\left[-\varepsilon b_{j k}\right]_{+} \beta_{j} & i=k \\
\beta_{i} & i \neq k,\end{cases} \\
& \gamma_{i}^{\prime}= \begin{cases}-\gamma_{k} & i=k \\
\gamma_{i}+\left[\varepsilon b_{k i}\right]_{+} \gamma_{k} & i \neq k .\end{cases}
\end{aligned}
$$

In parallel to $Q^{(\varepsilon \delta)}(z, \eta)$, we introduce

$$
e^{W_{i}^{\prime}}=e^{W_{\beta_{i}^{\prime}}^{(-\varepsilon \delta)}}, \quad e^{V_{i}^{\prime}}=e^{V_{\gamma_{i}^{\prime}}^{(-\varepsilon \delta)}}, \quad e^{v_{i}^{\prime}}=e^{\eta v_{\gamma_{i}^{\prime}}^{(-\varepsilon \delta)}} .
$$

Now we present the mutation formula of the Voros symbols for the signed flips. 
Theorem 7.5 (Mutation formula of the Voros symbols for the signed flip $\left.\mu_{k}^{(\varepsilon)}\right)$. For $i=1, \ldots, n$, we have

$$
\begin{aligned}
& \lim _{\delta \rightarrow+0} \mathcal{S}\left[e^{v_{i}^{\prime}}\right]= \begin{cases}\lim _{\delta \rightarrow+0} \mathcal{S}\left[\left(e^{v_{k}}\right)^{-1}\right] & i=k \\
\lim _{\delta \rightarrow+0} \mathcal{S}\left[e^{v_{i}}\left(e^{v_{k}}\right)^{\left[\varepsilon b_{k i}\right]_{+}}\right] & i \neq k,\end{cases} \\
& \lim _{\delta \rightarrow+0} \mathcal{S}\left[e^{W_{i}^{\prime}}\right]= \begin{cases}\lim _{\delta \rightarrow+0} \mathcal{S}\left[\left(e^{W_{k}}\right)^{-1}\left(\prod_{j=1}^{n}\left(e^{W_{j}}\right)^{\left[-\varepsilon b_{j k}\right]_{+}}\right)\left(1+\left(e^{V_{k}}\right)^{\varepsilon}\right)\right] & i=k \\
\lim _{\delta \rightarrow+0} \mathcal{S}\left[e^{W_{i}}\right] & i \neq k,\end{cases} \\
& \lim _{\delta \rightarrow+0} \mathcal{S}\left[e^{\left.V_{i}^{\prime}\right]}= \begin{cases}\lim _{\delta \rightarrow+0} \mathcal{S}\left[\left(e^{V_{k}}\right)^{-1}\right] & i \neq k . \\
\lim _{\delta \rightarrow+0} \mathcal{S}\left[e^{V_{i}}\left(e^{V_{k}}\right)^{\left[\varepsilon b_{k i}\right]_{+}}\left(1+\left(e^{V_{k}}\right)^{\varepsilon}\right)^{-b_{k i}}\right] & \end{cases} \right.
\end{aligned}
$$

Proof. Let us show (7.12). By (7.10),

$$
e^{v_{i}^{\prime}}=e^{\eta v_{\gamma_{i}^{\prime}}^{(-\varepsilon \delta)}}= \begin{cases}\left(\left(e^{\eta v_{\gamma_{k}}^{(-\varepsilon \delta)}}\right)^{-1}\right. & i=k \\ e^{\eta v_{\gamma_{i}}^{(-\varepsilon \delta)}}\left(e^{\eta v_{\gamma_{k}}^{(-\varepsilon \delta)}}\right)^{\left[\varepsilon b_{j k}\right]+} & i \neq k,\end{cases}
$$

Applying the Borel resummation operator $\mathcal{S}$ to (7.15), taking the limit $\delta \rightarrow+0$, then using Lemma 7.4, we obtain (7.12). The equalities (7.13) and (7.14) are obtained in a similar way from (7.9), (7.10), Proposition [7.3, and the facts $\left\langle\gamma_{k}, \beta_{i}\right\rangle=\delta_{k i}$ and $\left(\gamma_{k}, \gamma_{i}\right)=b_{k i}$.

The formulas (7.12)-(7.14) coincide with the exchange relation of seeds in (4.20), (4.21), and (4.23) under the identification

$$
\begin{aligned}
\lim _{\delta \rightarrow+0} e^{v_{i}} & \leftrightarrow y_{i}, \\
\lim _{\delta \rightarrow+0} e^{W_{i}} & \leftrightarrow x_{i}, \\
\lim _{\delta \rightarrow+0} e^{V_{i}} & \leftrightarrow \hat{y}_{i} .
\end{aligned}
$$

We phrase this result as "by the signed flips the Voros symbols $x_{i}=e^{W_{i}}, \hat{y}_{i}=e^{V_{i}}$, together with $y_{i}=e^{v_{i}}$, mutate as the variables in seeds (in the sense of Section 4.6)". Observe that the monomial parts in the right hand sides of (17.12)-(17.14) have the geometric origin, while the non-monomial parts have the analytic origin, i.e., the Stokes phenomenon.

Next, we reformulate the above result in terms of the Stokes automorphisms as in Section 3 .

Let $\mathbb{V}=\mathbb{V}\left(Q^{(\varepsilon \delta)}(z, \eta)\right)$ be the the Voros field for the potential $Q^{(\varepsilon \delta)}(z, \eta)$, i.e., the rational function field of the Voros symbols $e^{W_{1}}, \ldots, e^{W_{n}}, e^{V_{1}}, \ldots, e^{V_{n}}$ over $\mathbb{Q}$. By Lemma 7.1, $\mathbb{V}$ is also generated by $e^{W_{1}}, \ldots, e^{W_{n}}, e^{v_{1}}, \ldots, e^{v_{n}}$. Similarly, let $\mathbb{V}^{\prime}=\mathbb{V}\left(Q^{(-\varepsilon \delta)}(z, \eta)\right)$ be the Voros field for the potential $Q^{(-\varepsilon \delta)}(z, \eta)$, which is the rational function field of the Voros symbols $e^{W_{1}^{\prime}}, \ldots, e^{W_{n}^{\prime}}, e^{V_{1}^{\prime}}, \ldots, e^{V_{n}^{\prime}}$. 
The isomorphisms of the homology groups $\tau_{G, G^{\prime}}$ and $\tau_{G, G^{\prime}}^{\vee}$ in Proposition 6.28 (a) induce the following field isomorphism $\tau_{\mathbb{V}, \mathbb{V}^{\prime}}^{*}: \mathbb{V}^{\prime} \rightarrow \mathbb{V}$ :

$$
\begin{aligned}
\tau_{\mathbb{V}, \mathbb{V}^{\prime}}^{*}\left(e^{v_{i}^{\prime}}\right) & = \begin{cases}\left(e^{v_{k}}\right)^{-1} & i=k \\
e^{v_{i}}\left(e^{v_{k}}\right)^{\left[\varepsilon b_{k i}\right]_{+}} & i \neq k,\end{cases} \\
\tau_{\mathbb{V}, \mathbb{V}^{\prime}}^{*}\left(e^{W_{i}^{\prime}}\right) & = \begin{cases}\left(e^{W_{k}}\right)^{-1} \prod_{j=1}^{n}\left(e^{W_{j}}\right)^{\left[-\varepsilon b_{j k}\right]_{+}} & i=k \\
e^{W_{i}} & i \neq k .\end{cases}
\end{aligned}
$$

Compare them with (4.20) and (4.21). By (4.5), (7.17), (7.18), and Lemma 7.1, we have

$$
\tau_{\mathbb{V}, \mathbb{V}^{\prime}}^{*}\left(e^{V_{i}^{\prime}}\right)= \begin{cases}\left(e^{V_{k}}\right)^{-1} & i=k \\ e^{V_{i}}\left(e^{V_{k}}\right)^{\left[\varepsilon b_{k i}\right]_{+}} & i \neq k\end{cases}
$$

Also, in view of Proposition 7.3 and Lemma 7.4, we introduce the field automorphism $\mathfrak{S}_{\mathbb{V}, k}^{(\varepsilon)}: \mathbb{V} \rightarrow \mathbb{V}$ as follows.

$$
\begin{aligned}
\mathfrak{S}_{\mathbb{V}, k}^{(\varepsilon)}\left(e^{v_{i}}\right) & =e^{v_{i}} \\
\mathfrak{S}_{\mathbb{V}, k}^{(\varepsilon)}\left(e^{W_{i}}\right) & =e^{W_{i}}\left(1+\left(e^{V_{k}}\right)^{\varepsilon}\right)^{-\delta_{k i}} .
\end{aligned}
$$

By (7.20), (7.21), and Lemma 7.1, we have

$$
\mathfrak{S}_{\mathbb{V}, k}^{(\varepsilon)}\left(e^{V_{i}}\right)=e^{V_{i}}\left(1+\left(e^{V_{k}}\right)^{\varepsilon}\right)^{-b_{k i}} .
$$

We call $\mathfrak{S}_{\mathbb{V}, k}^{(\varepsilon)}$ the Stokes automorphism associated with the signed flip $\mu_{k}^{(\varepsilon)}$.

For simplicity, let us denote

$$
\begin{aligned}
& y_{i}=e^{v_{i}}, \quad x_{i}=e^{W_{i}}, \quad \hat{y}_{i}=e^{V_{i}}, \\
& y_{i}^{\prime}=e^{v_{i}^{\prime}}, \quad x_{i}^{\prime}=e^{W_{i}^{\prime}}, \quad \hat{y}_{i}^{\prime}=e^{V_{i}^{\prime}} .
\end{aligned}
$$

Then, it is easy to check that the following formulas hold.

$$
\begin{aligned}
& \left(\mathfrak{S}_{\mathbb{V}, k}^{(\varepsilon)} \circ \tau_{\mathbb{V}, \mathbb{V}^{\prime}}^{*}\right)\left(y_{i}^{\prime}\right)= \begin{cases}y_{k}^{-1} & i=k \\
y_{i} y_{k}\left[\varepsilon b_{k i}\right]_{+} & i \neq k,\end{cases} \\
& \left(\mathfrak{S}_{\mathbb{V}, k}^{(\varepsilon)} \circ \tau_{\mathbb{V}, \mathbb{V}^{\prime}}^{*}\right)\left(x_{i}^{\prime}\right)= \begin{cases}x_{k}{ }^{-1}\left(\prod_{j=1}^{n} x_{j}{ }^{\left[-\varepsilon b_{j k}\right]_{+}}\right)\left(1+\hat{y}_{k}{ }^{\varepsilon}\right) & i=k \\
x_{i} & i \neq k,\end{cases} \\
& \left(\mathfrak{S}_{\mathbb{V}, k}^{(\varepsilon)} \circ \tau_{\mathbb{V}, \mathbb{V}^{\prime}}^{*}\right)\left(\hat{y}_{i}^{\prime}\right)= \begin{cases}\hat{y}_{k}{ }^{-1} & i=k \\
\hat{y}_{i} \hat{y}_{k}\left[\varepsilon b_{k i}\right]_{+}\left(1+\hat{y}_{k}{ }^{\varepsilon}\right)^{-b_{k i}} & i \neq k .\end{cases}
\end{aligned}
$$

Theorem 7.5 is compactly expressed in the following way, where $\circ$ is the composition of operations. 

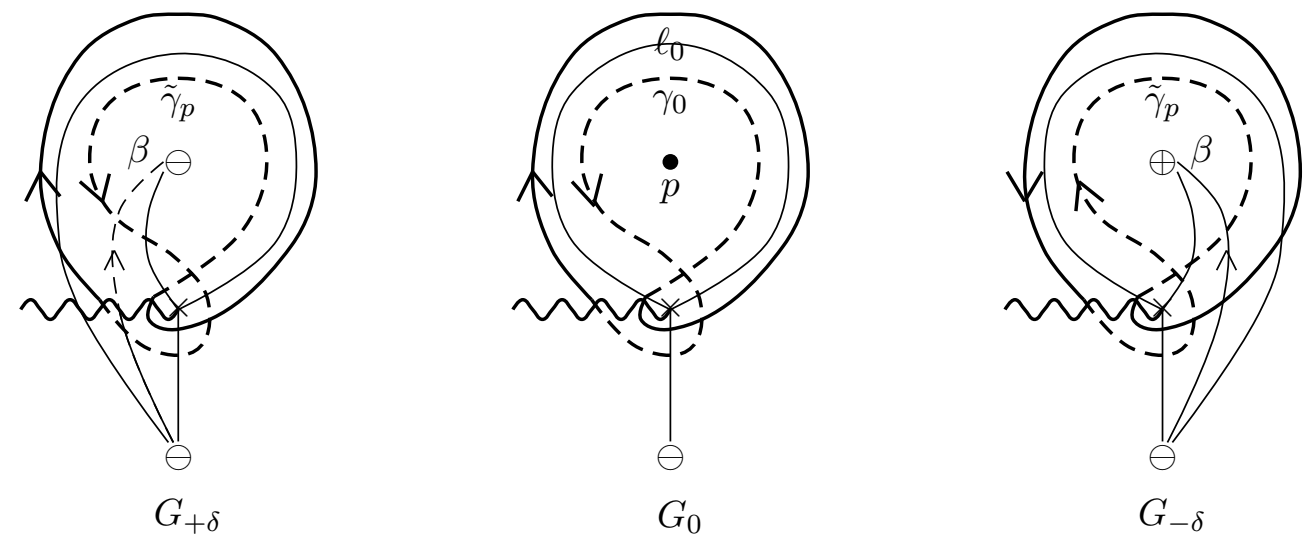

Figure 47. Cycles $\gamma_{0}$ and $\tilde{\gamma}_{p}$.

Theorem 7.6. The following equality of operations holds:

$$
\lim _{\delta \rightarrow+0} \circ \mathcal{S}=\lim _{\delta \rightarrow+0} \circ \mathcal{S} \circ \mathfrak{S}_{\mathbb{V}, k}^{(\varepsilon)} \circ \tau_{\mathbb{V}, \mathbb{V}^{\prime}}^{*}
$$

The formulation by the Stokes automorphisms enables us to treat the Stokes phenomenon more algebraically, and it will be useful when we study its global property in Section 8 ,

\subsection{Mutation formula of Voros symbols for signed pops}

Next we consider the case where the quadratic differential $\phi$ in Section 7.1 has a unique degenerate saddle trajectory $\ell_{0}$. Again, we choose a sufficiently small $\delta>0$ such that $G_{+\delta}=G\left(\phi_{+\delta}\right)$ and $G_{-\delta}=G\left(\phi_{-\delta}\right)$ are the saddle reductions of $G_{0}=G(\phi)$. Let us fix a $\operatorname{sign} \varepsilon= \pm$, and we set $G=G_{\varepsilon \delta}$ and $G^{\prime}=G_{-\varepsilon \delta}$. We assume that $G$ and $G^{\prime}$ are labeled so that they are related by the signed pop $G^{\prime}=\kappa_{p}^{(\varepsilon)}(G)$ at the double pole $p$ surrounded by $\ell_{0}$. See Figure 34. The story is quite parallel to the case of the signed flips, so we concentrate on the points which are special for this case.

Let $\gamma_{0} \in \Gamma_{G}$ be the saddle class associated with $\ell_{0}$ defined in Section 3.2. Let $\tilde{\gamma}_{p} \in \Gamma_{G}$ be the cycle given in Figure 47. Namely, $\tilde{\gamma}_{p}$ is $\gamma_{0}$ or $-\gamma_{0}$, and its orientation is determined by the condition $\left\langle\tilde{\gamma}_{p}, \beta\right\rangle=1$, where $\beta$ is any trajectory in the degenerate horizontal strip surrounding $p$ whose orientation is given as in Section 2.7.

We have the counterpart of Lemma 7.2 .

Lemma 7.7. The saddle class $\gamma_{0}$ coincides with $\varepsilon \tilde{\gamma}_{p}$.

Proof. This is clear from Figure 47.

Let us use the notation (7.1) for the Voros symbols for $Q^{(\varepsilon \delta)}(z, \eta)$.

The counterpart of Proposition 7.3 is as follows. 
Proposition 7.8. For any path $\beta \in \Gamma_{G}^{\vee}$ and any cycle $\gamma \in \Gamma_{G}$, we have

$$
\begin{aligned}
\lim _{\delta \rightarrow+0} \mathcal{S}\left[e^{W_{\beta}^{(-\varepsilon \delta)}}\right] & =\lim _{\delta \rightarrow+0} \mathcal{S}\left[e^{W_{\beta}^{(\varepsilon \delta)}}\left(1-\left(e^{V_{\tilde{\gamma} p}^{(\varepsilon \delta)}}\right)^{\varepsilon}\right)^{\left\langle\tilde{\gamma}_{p}, \beta\right\rangle}\right], \\
\lim _{\delta \rightarrow+0} \mathcal{S}\left[e^{V_{\gamma}^{(-\varepsilon \delta)}}\right] & =\lim _{\delta \rightarrow+0} \mathcal{S}\left[e^{V_{\gamma}^{(\varepsilon \delta)}}\right] .
\end{aligned}
$$

Proof. The first formula follows from Theorem 3.10 (b) and Lemma 7.7 by the same argument for Proposition 7.3 . The second formula is the same one in Theorem 3.10 (b).

Let us examine the integral $V_{\tilde{\gamma}_{p}}^{(\varepsilon \delta)}$ appearing in (17.29). As shown in (3.14), we have

$$
V_{\tilde{\gamma}_{p}}^{(\varepsilon \delta)}=\oint_{\tilde{\gamma}_{p}} \eta \sqrt{Q_{0}^{(\varepsilon \delta)}(z)} d z .
$$

Furthermore, we see in Figure 47 that the cycle $\tilde{\gamma}_{p}$ winds around $p$ anticlockwise and twice (modulo the $*$-equivalence) on the sheet where $p$ has the sign $\oplus$ with respect to the integral of $\sqrt{Q_{0}^{(\varepsilon \delta)}(z)}$. Thus, the right hand side of (17.31) equals to

$$
4 \pi i \eta \operatorname{Res}_{z=p_{\oplus}} \sqrt{Q_{0}^{(\varepsilon \delta)}(z)} d z
$$

where $z=p_{\oplus}$ implies taking the residue at $p$ on the above mentioned sheet.

More generally, we define, for any double pole $q$ of $Q_{0}^{(\varepsilon \delta)}(z)$, which is also a double pole of $Q_{0}^{(-\varepsilon \delta)}(z)$,

$$
\begin{aligned}
& \tilde{v}_{q}=4 \pi i \eta \operatorname{Res}_{z=q_{\oplus}} \sqrt{Q_{0}^{(\varepsilon \delta)}(z)} d z, \\
& \tilde{v}_{q}^{\prime}=4 \pi i \eta \operatorname{Res}_{z=q_{\oplus}} \sqrt{Q_{0}^{(-\varepsilon \delta)}(z)} d z .
\end{aligned}
$$

The definition makes sense, because $G$ and $G^{\prime}$ are saddle-free, so that $q$ has the definite sign on each sheet with respect to the corresponding integral.

Remark 7.9. The integral $\tilde{v}_{q}$ coincides with the residue at $q$ in [BS13, Section 2.4] up to the sign.

Now we present the mutation formula of the Voros symbols for the signed pops, where we use the notations (77.1), (7.2), and (7.11).

Theorem 7.10 (Mutation formula of the Voros symbols for the signed pop $\kappa_{p}^{(\varepsilon)}$ ). For $i=1, \ldots, n$ and any double pole $q$ of $G$, we have

$$
\begin{aligned}
\lim _{\delta \rightarrow+0} \mathcal{S}\left[e^{v_{i}^{\prime}}\right] & =\lim _{\delta \rightarrow+0} \mathcal{S}\left[e^{v_{i}}\right] \\
\lim _{\delta \rightarrow+0} \mathcal{S}\left[e^{\tilde{v}_{q}^{\prime}}\right] & =\lim _{\delta \rightarrow+0} \mathcal{S}\left[\left(e^{\tilde{v}_{q}}\right)^{1-2 \delta_{q p}}\right] \\
\lim _{\delta \rightarrow+0} \mathcal{S}\left[e^{W_{i}^{\prime}}\right] & =\lim _{\delta \rightarrow+0} \mathcal{S}\left[e^{W_{i}}\left(1-\left(e^{\tilde{v}_{p}}\right)^{\varepsilon}\right)^{\delta_{i i_{p}}-\delta_{i j_{p}}}\right], \\
\lim _{\delta \rightarrow+0} \mathcal{S}\left[e^{V_{i}^{\prime}}\right] & =\lim _{\delta \rightarrow+0} \mathcal{S}\left[e^{V_{i}}\right]
\end{aligned}
$$

where $i_{p}$ and $j_{p}$ are the labels in (5.6). 
Proof. The formulas (7.35), (7.37), and (7.38) are obtained from Propositions 6.28 (b) and 7.8, and the facts $\left\langle\tilde{\gamma}_{p}, \beta_{i_{p}}\right\rangle=1,\left\langle\tilde{\gamma}_{p}, \beta_{j_{p}}\right\rangle=-1$ in the same way as the proof of Theorem 7.5. Let us prove (7.36). First, we consider the nontrivial case $q=p$. By Lemma 7.7, the cycle $\tilde{\gamma}_{p}^{\prime}$ for $G^{\prime}$ is related to $\tilde{\gamma}_{p}$ for $G$ as $\tilde{\gamma}_{p}^{\prime}=-\tilde{\gamma}_{p}$. Thus,

$$
e^{\tilde{v}_{p}^{\prime}}=e^{\eta V_{\tilde{\gamma}_{p}^{\prime}}^{(-\varepsilon \delta)}}=e^{-\eta V_{\tilde{\gamma}_{p}}^{(-\varepsilon \delta)}} .
$$

By (7.31), there is no jump between $V_{\tilde{\gamma}_{p}}^{(-\varepsilon \delta)}$ and $V_{\tilde{\gamma}_{p}}^{(\varepsilon \delta)}$ for $\delta \rightarrow+0$. Thus, we have

$$
\lim _{\delta \rightarrow+0} \mathcal{S}\left[e^{-\eta V_{\tilde{\gamma}_{p}}^{(-\varepsilon \delta)}}\right]=\lim _{\delta \rightarrow+0} \mathcal{S}\left[e^{-\eta V_{\tilde{\gamma}_{p}}^{(\varepsilon \delta)}}\right]=\lim _{\delta \rightarrow+0} \mathcal{S}\left[\left(e^{\tilde{v}_{p}}\right)^{-1}\right]
$$

Let us consider the case $q \neq p$. Since we assume that $G_{0}$ has no saddle trajectory other than $\ell_{0}$, the sign $\oplus / \ominus$ of $q$ does not change under the signed pop $\kappa_{p}^{(\varepsilon)}$. Thus, we have $\lim _{\delta \rightarrow+0} \tilde{v}_{q}^{\prime}=\lim _{\delta \rightarrow+0} \tilde{v}_{q}$, and the equality (7.36) follows.

We note that the mutation of $\tilde{y}_{q}=e^{\tilde{v}_{q}}$ by the signed flips in Section 7.1 is trivial. Thus, summarizing Theorems 7.5 and 7.10, we obtain our first main result.

Theorem 7.11. By the signed flips and the signed pops, the Voros symbols $x_{i}=e^{W_{i}}$ and $\hat{y}_{i}=e^{V_{i}}$, together with $y_{i}=e^{v_{i}}$ and $\tilde{y}_{q}=e^{\tilde{v}_{q}}$, mutate as the variables of extended seeds (in the sense of Section 5.5).

In the same spirit of Section 7.1, we reformulate Theorem 7.10 in terms of the Stokes automorphisms for the signed pops.

Let us $\tilde{\mathbb{V}}=\tilde{\mathbb{V}}\left(Q^{(\varepsilon \delta)}(z, \eta)\right)$ denote the extension of the Voros field $\mathbb{V}=\mathbb{V}\left(Q^{(\varepsilon \delta)}(z, \eta)\right)$ in Section 7.1 by $e^{\tilde{v}_{q}}$ 's. We call $\tilde{\mathbb{V}}$ the extended Voros field of $Q^{(\varepsilon \delta)}(z, \eta)$. We define $\tilde{\mathbb{V}}^{\prime}=\tilde{\mathbb{V}}\left(Q^{(-\varepsilon \delta)}(z, \eta)\right)$ in the same way.

Again, the isomorphisms of the homology groups $\tau_{G, G^{\prime}}$ and $\tau_{G, G^{\prime}}^{\vee}$ in Proposition 6.28 (b) induce the following field isomorphism $\tau_{\tilde{\mathbb{V}}, \tilde{\mathbb{V}}^{\prime}}^{*}: \tilde{\mathbb{V}}^{\prime} \rightarrow \tilde{\mathbb{V}}$ :

$$
\tau_{\widetilde{\mathbb{V}}, \tilde{\mathbb{V}}^{\prime}}^{*}\left(e^{v_{i}^{\prime}}\right)=e^{v_{i}}, \quad \tau_{\widetilde{\mathbb{V}}, \tilde{\mathbb{V}}^{\prime}}^{*}\left(e^{\tilde{e}_{q}^{\prime}}\right)=\left(e^{\tilde{v}_{q}}\right)^{1-2 \delta_{p q}}, \quad \tau_{\widetilde{\mathbb{V}}, \tilde{\mathbb{V}}^{\prime}}^{*}\left(e^{W_{i}^{\prime}}\right)=e^{W_{i}}
$$

Here we use the same symbol for the isomorphism in (17.17) and (17.17), since the both are By Lemma 7.1, we have

$$
\tau_{\tilde{\mathbb{V}}, \tilde{\mathbb{V}}^{\prime}}^{*}\left(e^{V_{i}^{\prime}}\right)=e^{V_{i}}
$$

We also introduce the field automorphism $\mathfrak{K}_{\tilde{\mathbb{V}}, p}^{(\varepsilon)}: \tilde{\mathbb{V}} \rightarrow \tilde{\mathbb{V}}$ as follows.

$$
\begin{aligned}
\mathfrak{K}_{\tilde{\mathbb{V}}, p}^{(\varepsilon)}\left(e^{v_{i}}\right) & =e^{v_{i}} \\
\mathfrak{K}_{\tilde{\mathbb{V}}, p}^{(\varepsilon)}\left(e^{\tilde{v}_{q}}\right) & =e^{\tilde{v}_{q}} \\
\mathfrak{K}_{\tilde{\mathbb{V}}, p}^{(\varepsilon)}\left(e^{W_{i}}\right) & =e^{W_{i}}\left(1-\left(e^{\tilde{v}_{p}}\right)^{\varepsilon}\right)^{\delta_{i i_{p}}-\delta_{i j_{p}}} .
\end{aligned}
$$


By Lemma 7.1, we have

$$
\mathfrak{K}_{\tilde{\mathbb{V}}, p}^{(\varepsilon)}\left(e^{V_{i}}\right)=e^{V_{i}}
$$

We call $\mathfrak{K}_{\tilde{\mathbb{V}}, p}^{(\varepsilon)}$ the Stokes automorphism associated with the signed pop $\kappa_{p}^{(\varepsilon)}$.

We denote

$$
\begin{array}{llll}
y_{i}=e^{v_{i}}, & \tilde{y}_{q}=e^{\tilde{v}_{q}}, & x_{i}=e^{W_{i}}, & \hat{y}_{i}=e^{V_{i}}, \\
y_{i}^{\prime}=e^{v_{i}^{\prime}}, & \tilde{y}_{q}^{\prime}=e^{\tilde{v}_{q}^{\prime}}, & x_{i}^{\prime}=e^{W_{i}^{\prime}}, & \hat{y}_{i}^{\prime}=e^{V_{i}^{\prime}} .
\end{array}
$$

Then, it is easy to check that the following formulas hold.

$$
\begin{aligned}
& \left(\mathfrak{K}_{\tilde{\mathbb{V}}, p}^{(\varepsilon)} \circ \tau_{\tilde{\mathbb{V}}, \tilde{\mathbb{V}}^{\prime}}^{*}\right)\left(y_{i}^{\prime}\right)=y_{i}, \\
& \left(\mathfrak{K}_{\tilde{\mathbb{V}}, p}^{(\varepsilon)} \circ \tau_{\tilde{\mathbb{V}}, \tilde{\mathbb{V}}^{\prime}}^{*}\right)\left(\tilde{y}_{q}^{\prime}\right)=\tilde{y}_{q}{ }^{1-2 \delta_{q p}}, \\
& \left(\mathfrak{K}_{\tilde{\mathbb{V}}, p}^{(\varepsilon)} \circ \tau_{\tilde{\mathbb{V}}, \tilde{\mathbb{V}}^{\prime}}^{*}\right)\left(x_{i}^{\prime}\right)=x_{i}\left(1-\left(\tilde{y}_{p}\right)^{\varepsilon}\right)^{\delta_{i i_{p}}-\delta_{i j_{p}},} \\
& \left(\mathfrak{K}_{\tilde{\mathbb{V}}, p}^{(\varepsilon)} \circ \tau_{\tilde{\mathbb{V}}, \tilde{\mathbb{V}}^{\prime}}^{*}\right)\left(\hat{y}_{i}^{\prime}\right)=\hat{y}_{i} .
\end{aligned}
$$

Theorem 7.10 is compactly expressed in the following way.

Theorem 7.12. The following equality of operations holds:

$$
\lim _{\delta \rightarrow+0} \circ \mathcal{S}=\lim _{\delta \rightarrow+0} \circ \mathcal{S} \circ \mathfrak{K}_{\tilde{\mathbb{V}}, p}^{(\varepsilon)} \circ \tau_{\mathbb{\mathrm { V }}, \tilde{\mathbb{V}}^{\prime}}^{*}
$$

For the completeness, we also extend the isomorphisms $\tau_{\mathbb{V}, \mathbb{V}^{\prime}}^{*}: \mathbb{V}^{\prime} \rightarrow \mathbb{V}$ and $\mathfrak{S}_{\mathbb{V}, k}^{(\varepsilon)}: \mathbb{V} \rightarrow \mathbb{V}$ for the signed flips in Section 7.1 to the ones $\tau_{\tilde{\mathbb{V}}, \tilde{\mathbb{V}}^{\prime}}^{*}: \tilde{\mathbb{V}}^{\prime} \rightarrow \tilde{\mathbb{V}}$ and $\mathfrak{S}_{\tilde{\mathbb{V}}, k}^{(\varepsilon)}: \tilde{\mathbb{V}} \rightarrow \tilde{\mathbb{V}}$ in a trivial way:

$$
\tau_{\tilde{\mathbb{V}}, \tilde{\mathbb{V}}^{\prime}}^{*}\left(\tilde{y}_{q}^{\prime}\right)=\tilde{y}_{q}, \quad \mathfrak{S}_{\tilde{\mathbb{V}}, k}^{(\varepsilon)}\left(\tilde{y}_{q}\right)=\tilde{y}_{q}
$$

Then, we have

$$
\left(\mathfrak{S}_{\tilde{\mathbb{V}}, k}^{(\varepsilon)} \circ \tau_{\tilde{\mathbb{V}}, \tilde{\mathbb{V}}^{\prime}}^{*}\right)\left(\tilde{y}_{q}^{\prime}\right)=\tilde{y}_{q},
$$

and Theorem 7.6 still holds.

\section{Application: Identities of Stokes automorphisms}

By combining all results in the previous sections we derive the identities of Stokes automorphisms associated with periods of seeds in cluster algebras.

\subsection{Regular deformation and mutation of potentials}

In Section 6 we introduced regular deformations and signed mutations for Stokes graphs of Schrödinger equations. Here we extend them to potential functions. 
Definition 8.1. We say that the potential $Q(z, \eta)$ of a Schrödinger equation (2.1) is saddle-free if its Stokes graph is saddle-free. For a pair of two saddle-free potentials $Q(z, \eta)$ and $Q^{\prime}(z, \eta)$, we say that they are related by a regular deformation of potentials if there exists a family of potentials

$$
Q(z, \eta ; t)=\sum_{n=0}^{\infty} \eta^{-n} Q_{n}(z ; t) \quad(0 \leq t \leq 1)
$$

satisfying the following conditions:

- $Q(z, \eta ; t)$ is a polynomial in $\eta^{-1}$ (i.e., $Q_{n}(z ; t)=0$ for $\left.n \gg 1\right)$. Each coefficient $Q_{n}(z ; t)$ is analytic in $t$ and satisfies $Q(z, \eta)=Q(z, \eta ; 0)$ and $Q^{\prime}(z, \eta)=Q(z, \eta ; 1)$.

- For any $t, Q(z, \eta ; t)$ satisfies Assumption 2.3 and 2.4. Moreover, for any $n \geq 0$, the pole orders of $Q_{n}(z ; t)$ are independent of $t$.

- The family $\left\{\phi_{t}=Q_{0}(z ; t) d z^{\otimes 2} \mid 0 \leq t \leq 1\right\}$ satisfies Condition 6.15,

Let $S_{\text {odd }}(z, \eta ; t)$ be the formal series (2.14) defined from the potential $Q(z, \eta ; t)$ satisfying the above conditions. Since the coefficients of $S_{\text {odd }}(z, \eta ; t)$ are determined by the recursion relation (2.12), the coefficients of $S_{\text {odd }}(z, \eta ; t)$ are analytic not only in $z$ but also in $t$ as long as $S_{-1}(z ; t)=\sqrt{Q_{0}(z ; t)}$ never vanishes. Namely, they are analytic in $t$ as long as zeros and poles of $\phi_{t}$ (which may depend on $t$ ) do not coincides with $z$. Moreover, each coefficient of the Voros symbols are also analytic in $t$ since Condition 6.15 guarantees that zeros and poles of $\phi_{t}$ never confluence under a regular deformation. Note that, if a pair of two zeros (or a pair of a zero and a pole) of $\phi_{t}$ merges and some path which defines a Voros symbol is pinched by the merging pair at $t=t_{0}$ with some $t_{0} \in[0,1]$, then the coefficients of the Voros symbols may not be analytic in $t$ at the point $t=t_{0}$ since the integrand (i.e., coefficients of $\left.S_{\text {odd }}(z, \eta ; t)\right)$ may have singularities at zeros and poles. However, Condition 6.15 guarantees that such a point $t_{0}$ never appears in a regular deformation.

Remark 8.2. Since the Stokes graphs of the Schrödinger equations whose potentials are given by (8.1) are saddle-free for any $t \in[0,1]$, the Voros symbols are Borel summable for any $t \in[0,1]$. Therefore, we expect that no Stokes phenomenon occurs to the Voros symbols under the regular deformation of potentials. In other words, for any $0 \leq t_{0} \leq 1$, we conjecture that the following equalities hold:

$$
\lim _{t \rightarrow t_{0}} \mathcal{S}\left[e^{W_{\beta}(t)}\right]=\mathcal{S}\left[e^{W_{\beta}\left(t_{0}\right)}\right], \quad \lim _{t \rightarrow t_{0}} \mathcal{S}\left[e^{V_{\gamma}(t)}\right]=\mathcal{S}\left[e^{V_{\gamma}\left(t_{0}\right)}\right]
$$

Here $e^{W_{\beta}(t)}$ and $e^{V_{\gamma}(t)}$ denote the Voros symbols for the potential $Q(z, \eta ; t)$, and we have identified the paths and cycles by the isomorphism (6.8) induced by the regular deformation of the quadratic differentials $\phi_{t}$. Under the conjecture, we can identify the Voros fields $\mathbb{V}=\mathbb{V}(Q(z, \eta))$ and $\mathbb{V}^{\prime}=\mathbb{V}\left(Q^{\prime}(z, \eta)\right)$ by the natural isomorphism $e^{W_{i}} \mapsto e^{W_{i}^{\prime}}, e^{V_{i}} \mapsto e^{V_{i}^{\prime}}$, if they are related by a regular deformation of potentials. See also Remark 8.5 later. 
Next we introduce the signed mutations of potentials.

Definition 8.3. Let $Q(z, \eta)$ and $Q^{\prime}(z, \eta)$ be a pair of saddle-free potentials, and let $G$ and $G^{\prime}$ be their labeled Stokes graphs. Fix a $\operatorname{sign} \varepsilon= \pm$. Suppose that there exists a potential $Q^{(0)}(z, \eta)$ satisfying the following conditions:

- The Stokes graph $G_{0}=G\left(\phi_{0}\right)$ has a unique regular saddle trajectory, where $\phi_{0}$ is the quadratic differential associated with the potential $Q^{(0)}(z, \eta)$.

- Let $Q^{(\theta)}(z, \eta)$ be the $S^{1}$-family for the potential $Q^{(0)}(z, \eta)$, and let $\phi_{\theta}$ be the quadratic differential associated with $Q^{(\theta)}(z, \eta)$. Choose a sufficiently small $\delta>0$ such that $G_{\varepsilon \delta}=G\left(\phi_{\varepsilon \delta}\right)$ and $G_{-\varepsilon \delta}=G\left(\phi_{-\varepsilon \delta}\right)$ are the saddle reductions of $G_{0}$. Then, the potentials $Q(z, \eta)$ and $Q^{(+\varepsilon \delta)}(z, \eta)$ (resp., $Q^{\prime}(z, \eta)$ and $Q^{(-\varepsilon \delta)}(z, \eta)$ ) are related by a regular deformation of potentials.

- The labeled Stokes graphs $G$ and $G^{\prime}$ are related by the signed flip $G^{\prime}=\mu_{k}^{(\varepsilon)}(G)$ in the sense of Definition 6.18.

Then, we write $Q^{\prime}(z, \eta)=\mu_{k}^{(\varepsilon)}(Q(z, \eta))$, assuming the labels of $G$ and $G^{\prime}$. This defines the signed flip $\mu_{k}^{(\varepsilon)}$ for potentials. The signed pop $\kappa_{p}^{(\varepsilon)}$ for potentials is defined by the same manner by considering "degenerate saddle trajectory" instead of "regular saddle trajectory" in the above.

\subsection{Stokes automorphism for general cycle}

Let $Q(z, \eta)$ be a saddle-free potential, and let $G$ be its labeled Stokes graph. Let $\mathbb{V}=\mathbb{V}(Q(z, \eta))$ be the Voros field of $Q(z, \eta)$. For any cycle $\gamma \in \Gamma_{G}, k=1, \ldots, n$, and $\operatorname{sign} \varepsilon$, we define the field automorphism $\mathfrak{S}_{\mathbb{V}, \gamma}^{(\varepsilon)}: \mathbb{V} \rightarrow \mathbb{V}$ as follows.

$$
\begin{aligned}
\mathfrak{S}_{\mathbb{V}, \gamma}^{(\varepsilon)}\left(e^{v_{i}}\right) & =e^{v_{i}}, \\
\mathfrak{S}_{\mathbb{V}, \gamma}^{(\varepsilon)}\left(e^{W_{i}}\right) & =e^{W_{i}}\left(1+\left(e^{V_{\gamma}}\right)^{\varepsilon}\right)^{-\left\langle\gamma, \beta_{i}\right\rangle} .
\end{aligned}
$$

Thanks to Proposition 6.27 and Lemma 7.1, the following formula holds.

$$
\mathfrak{S}_{\mathbb{V}, \gamma}^{(\varepsilon)}\left(e^{V_{i}}\right)=e^{V_{i}}\left(1+\left(e^{V_{\gamma}}\right)^{\varepsilon}\right)^{-\left(\gamma, \gamma_{i}\right)} .
$$

If we set $\gamma=\gamma_{k}$, these formulas reduce to those of the "original" Stokes automorphism $\mathfrak{S}_{\mathbb{V}, k}^{(\varepsilon)}$ for the signed flip $\mu_{k}^{(\varepsilon)}$ in Section [7.1. We call $\mathfrak{S}_{\mathbb{V}, \gamma}^{(\varepsilon)}$ the Stokes automorphism for a cycle $\gamma$ with sign $\varepsilon$. We note the equality

$$
\mathfrak{S}_{\mathbb{V}, \gamma}^{(-)}=\left(\mathfrak{S}_{\mathbb{V},-\gamma}^{(+)}\right)^{-1}
$$

Let $Q(z, \eta)$ and $Q^{\prime}(z, \eta)$ be a pair of saddle-free potentials, and let $G$ and $G^{\prime}$ be their labeled Stokes graphs. Suppose that $Q(z, \eta)$ and $Q^{\prime}(z, \eta)$ are related by

$$
Q^{\prime}(z, \eta)= \begin{cases}\mu_{k}^{\left(\varepsilon^{\prime}\right)}(Q(z, \eta)) & \text { if } \alpha_{k} \text { is flippable in } T \\ \left(\mu_{k}^{\left(\varepsilon^{\prime}\right)} \circ \kappa_{p}^{\left(\varepsilon^{\prime \prime}\right)}\right)(Q(z, \eta)) & \text { otherwise }\end{cases}
$$


where $T$ is the labeled Stokes triangulation for $G, p$ is the puncture inside the self-folded triangle in $T$ which $\alpha_{k}$ belongs to, and $\varepsilon^{\prime}$ and $\varepsilon^{\prime \prime}$ are any signs. Under this assumption, let $\tau_{G, G^{\prime}}: \Gamma_{G^{\prime}} \rightarrow \Gamma_{G}$ be the one in (6.10), where $\varepsilon$ therein is replaced with $\varepsilon^{\prime}$. Also, let $\tau_{\mathbb{V}, \mathbb{V}^{\prime}}^{*}: \mathbb{V}^{\prime} \rightarrow \mathbb{V}$ be the field automorphism from $\mathbb{V}=\mathbb{V}(Q(z, \eta))$ to $\mathbb{V}^{\prime}=\mathbb{V}\left(Q^{\prime}(z, \eta)\right)$ defined by (7.17) and (7.18), where $\varepsilon$ therein is replace with $\varepsilon^{\prime}$. In particular, they are independent of the $\operatorname{sign} \varepsilon^{\prime \prime}$.

Proposition 8.4. For any $\gamma^{\prime} \in \Gamma_{G^{\prime}}$, we have the following equality of isomorphisms from $\mathbb{V}^{\prime}$ to $\mathbb{V}$ :

$$
\tau_{\mathbb{V}, \mathbb{V}^{\prime}}^{*} \circ \mathfrak{S}_{\mathbb{V}^{\prime}, \gamma^{\prime}}^{(\varepsilon)}=\mathfrak{S}_{\mathbb{V}, \tau_{G, G^{\prime}}\left(\gamma^{\prime}\right)}^{(\varepsilon)} \circ \tau_{\mathbb{V}, \mathbb{V}^{\prime}}^{*}
$$

Here, the sign $\varepsilon$ for $\mathfrak{S}_{\mathbb{V}^{\prime}, \gamma^{\prime}}^{(\varepsilon)}$ and the sign $\varepsilon^{\prime}$ for $\tau_{\mathbb{V}, \mathbb{V}^{\prime}}^{*}$ are taken independently.

Proof. It is enough to show that the actions of both hand sides of (8.7) on $e^{W_{i}^{\prime}}$ coincide. By explicit calculation, this is equivalent to the condition

$$
\left\langle\tau_{G, G^{\prime}}\left(\gamma^{\prime}\right), \tau_{G, G^{\prime}}^{\vee}\left(\beta_{i}^{\prime}\right)\right\rangle=\left\langle\gamma^{\prime}, \beta_{i}^{\prime}\right\rangle
$$

This equality is known (e.g., [Nak12, Section 3.3]), and it is easily verified.

\subsection{Identities of Stokes automorphisms}

As the initial data we choose a saddle-free potential $Q^{0}(z, \eta)$ on a compact Riemann surface $\Sigma$. Let $G^{0}$ be its labeled Stokes graph, $T^{0}$ be the associated Stokes triangulation of the bordered surface $(\mathbf{S}, \mathbf{M}, \mathbf{A})$, and $B^{0}$ be the adjacency matrix of $T^{0}$.

We consider the cluster algebra with the initial seed $\left(B^{0}, x^{0}, y^{0}\right)$. Let $\vec{k}=$ $\left(k_{1}, \ldots, k_{N}\right)$ be a $\nu$-period of $\left(B^{0}, x^{0}, y^{0}\right)$. Recall that from the sequence of labeled seeds (6.12), we obtain the sequence of labeled Stokes triangulations (6.13), which further induces the sequence of labeled extended seeds (6.17) and the sequence of labeled Stokes graphs (6.19). We have the periodicity properties for them in Propositions 6.30 and 6.31.

Suppose that there is a sequence of deformations of potentials starting from $Q^{0}(z, \eta)$

$$
Q(z, \eta)(1)=Q^{0}(z, \eta) \stackrel{\tilde{\mu}_{k_{1}}^{\left(\varepsilon_{1}\right)}}{\rightarrow} Q(z, \eta)(2) \stackrel{\tilde{\mu}_{k_{2}}^{\left(\varepsilon_{2}\right)}}{\rightarrow} \cdots \stackrel{\tilde{\mu}_{\left.k_{N}\right)}^{\left(\varepsilon_{N}\right)}}{\rightarrow} Q(z, \eta)(N+1) \stackrel{\tilde{\kappa}}{\rightarrow} Q(z, \eta)(N+2),
$$

where the $\operatorname{sign} \varepsilon_{t}$ is the tropical sign of $y_{k_{t}}(t)$ for the sequence $(\underline{6.12})$, and $\tilde{\mu}_{k_{t}}^{\left(\varepsilon_{t}\right)}$ and $\tilde{\kappa}$ are the ones in (6.14) and (6.16) but for potentials.

By Theorem 7.11, the periodicity of the labeled extended seeds in (6.17) is realized by Stokes automorphisms and isomorphisms $\tau^{*}$ on the extended Voros fields $\tilde{\mathbb{V}}(t)$ for $Q(z, \eta)(t)$. Among them, the Stokes automorphisms for the signed pops induce the local rescaling. By Proposition 5.31 and the definition of $\tilde{\kappa}, \mathfrak{K}_{\tilde{\mathbb{V}}, p}^{(\varepsilon)}$ and $\mathfrak{K}_{\tilde{\mathrm{V}}, p}^{(-\varepsilon)}$ pairwise 
cancel and they are safely removed. Thus, we obtain the following identity of Stokes automorphisms and isomorphisms on the Voros fields $\mathbb{V}(t)$ for $Q(z, \eta)(t)$.

$$
\mathfrak{S}_{\mathbb{V}(1), \gamma_{k_{1}}(1)}^{\left(\varepsilon_{1}\right)} \tau_{\mathbb{V}(1), \mathbb{V}(2)}^{*} \mathfrak{S}_{\mathbb{V}(2), \gamma_{k_{2}}(2)}^{\left(\varepsilon_{2}\right)} \tau_{\mathbb{V}(2), \mathbb{V}(3)}^{*} \cdots \mathfrak{S}_{\mathbb{V}(N), \gamma_{k_{N}}(N)}^{\left(\varepsilon_{N}\right)} \tau_{\mathbb{V}(N), \mathbb{V}(N+1)}^{*}=\nu_{\mathbb{V}(1), \mathbb{V}(N+1)}^{*} .
$$

Here, the composition symbol $\circ$ is omitted, and $\nu_{\mathbb{V}(1), \mathbb{V}(N+1)}^{*}: \mathbb{V}(N+1) \rightarrow \mathbb{V}(1)$ is the isomorphism defined by $e^{v_{\nu(i)}(N+1)} \mapsto e^{v_{i}(1)}, e^{W_{\nu(i)}(N+1)} \mapsto e^{W_{i}(1)}$.

Remark 8.5. Assuming that the conjecture (8.2) holds. Then, the left hand side of the identity (8.10) faithfully expresses the formula describing the effect of all Stokes phenomena associated with the deformation sequence (8.9) of potentials (where the Stokes phenomena relevant to pops are canceled out). That is, the equality (8.10) has an analytic meaning. On the other hand, the equality (8.10) itself holds regardless of the validity of the conjecture (8.2) or even without the existence of the deformation sequence (8.9), since it expresses the periodicity of the labeled seeds in (6.12).

From the identity (8.10), one can derive the identity among Stokes automorphisms acting on the initial Voros field $\mathbb{V}(1)$ by pushing forward the Stokes automorphisms acting on the Voros fields $\mathbb{V}(t)$ for $t>1$. This is our second main result.

Theorem 8.6. The following identity holds.

$$
\mathfrak{S}_{\mathbb{V}(1), \gamma_{k_{1}}(1)}^{\left(\varepsilon_{1}\right)} \mathfrak{S}_{\mathbb{V}(1), \tau_{G(1), G(2)}^{\left(\varepsilon_{2}\right)}\left(\gamma_{k_{2}}(2)\right)} \cdots \mathfrak{S}_{\mathbb{V}(1), \tau_{G(1), G(N)}^{\left(\varepsilon_{N}\right)}\left(\gamma_{k_{N}}(N)\right)}=\mathrm{id},
$$

where $\tau_{G(1), G(t)}=\tau_{G(1), G(2)} \circ \tau_{G(2), G(3)} \cdots \circ \tau_{G(t-1), G(t)}$. Furthermore, let $c(t)=\left(c_{i}(t)\right)_{i=1}^{n}$ be the c-vector of $y_{k_{t}}(t)$ for the sequence (6.12) with respect to the initial $y$-variables $y_{i}(1)$, i.e.,

$$
\left[y_{k_{t}}(t)\right]=\sum_{i=1}^{n} y_{i}(1)^{c_{i}(t)}
$$

(See Section 4.6.) Then, the cycle $\tau_{G(1), G(t)}\left(\gamma_{k_{t}}(t)\right)$ therein is given by

$$
\tau_{G(1), G(t)}\left(\gamma_{k_{t}}(t)\right)=\sum_{i=1}^{n} c_{i}(t) \gamma_{i}(1) .
$$

Proof. We rewrite the left hand side of the identity (8.10) by repeated application of Proposition 8.4 in the following manner.

$$
\begin{aligned}
& \mathfrak{S}_{\mathbb{V}(1), \gamma_{k_{1}}(1)}^{\left(\varepsilon_{1}\right)} \tau_{\mathbb{V}(1), \mathbb{V}(2)}^{*} \mathfrak{S}_{\mathbb{V}(2), \gamma_{k_{2}}(2)}^{\left(\varepsilon_{2}\right)} \tau_{\mathbb{V}(2), \mathbb{V}(3)}^{*} \mathfrak{S}_{\mathbb{V}(3), \gamma_{k_{3}}(3)}^{\left(\varepsilon_{3}\right)} \tau_{\mathbb{V}(3), \mathbb{V}(4)}^{*} \cdots \\
& =\mathfrak{S}_{\mathbb{V}(1), \gamma_{k_{1}}(1)}^{\left(\varepsilon_{1}\right)} \mathfrak{S}_{\left.\mathbb{V}(1), \tau_{G(1), G(2)}^{\left(\varepsilon_{2}\right)} \gamma_{k_{2}}(2)\right)} \tau_{\mathbb{V}(1), \mathbb{V}(3)}^{*} \mathfrak{S}_{\mathbb{V}(3), \gamma_{k_{3}}(3)}^{\left(\varepsilon_{3}\right)} \tau_{\mathbb{V}(3), \mathbb{V}(4)}^{*} \cdots \\
& =\mathfrak{S}_{\mathbb{V}(1), \gamma_{k_{1}}(1)}^{\left(\varepsilon_{1}\right)} \mathfrak{S}_{\mathbb{V}(1), \tau_{G(1), G(2)}^{\left(\varepsilon_{2}\right)}\left(\gamma_{k_{2}}(2)\right)} \mathfrak{S}_{\mathbb{V}(1), \tau_{G(1), G(3)}\left(\gamma_{k_{3}}(3)\right)}^{\left(\varepsilon_{3}\right)} \tau_{\mathbb{V}(1), \mathbb{V}(4)}^{*} \cdots \\
& =\mathfrak{S}_{\mathbb{V}(1), \gamma_{k_{1}}(1)}^{\left(\varepsilon_{1}\right)} \mathfrak{S}_{\mathbb{V}(1), \tau_{G(1), G(2)}\left(\gamma_{k_{2}}(2)\right)}^{\left(\varepsilon_{2}\right)} \cdots \mathfrak{S}_{\mathbb{V}(1), \tau_{G(1), G(N)}\left(\gamma_{k_{N}}(N)\right)}^{\left(\varepsilon_{N}\right)} \tau_{\mathbb{V}(1), \mathbb{V}(N+1)}^{*} .
\end{aligned}
$$

Thanks to the choice of the sign $\varepsilon_{t}=\varepsilon\left(y_{k_{t}}(t)\right), \tau_{G(t), G(t+1)}$ acts as the mutation of the (logarithm of) tropical $y$-variables for the sequence (6.12). See the remark 
after Proposition 6.28. Thus, the claim (8.13) follows from (8.12). It also implies $\tau_{G(1), G(N+1)}\left(\gamma_{\nu(i)}\right)=\gamma_{i}$ due to the $\nu$-periodicity of the sequence (6.12). Therefore, we have $\tau_{\mathbb{V}(1), \mathbb{V}(N+1)}^{*}=\nu_{\mathbb{V}(1), \mathbb{V}(N+1)}^{*}$, which cancels the right hand side of the identity (8.10). Thus, we obtain the identity (8.11).

Remark 8.7. The derivation of the identity (8.11) is parallel to that of the quantum dilogarithm identities in [Kel11, KN11].

Example 8.8 (Pentagon relation (5)). Let $G=G(1)$ be the initial labeled Stokes graph in Figure 43, Let $\gamma_{1}$ and $\gamma_{2}$ be the simple cycles of $\Gamma_{G}$. Let $\mathbb{V}=\mathbb{V}(1)$ be the initial Voros field. With the data in (4.27) and (4.28), the identity (8.11) reads

$$
\mathfrak{S}_{\mathbb{V}, \gamma_{1}}^{(+)} \mathfrak{S}_{\mathbb{V}, \gamma_{1}+\gamma_{2}}^{(+)} \mathfrak{S}_{\mathbb{V}, \gamma_{2}}^{(+)} \mathfrak{S}_{\mathbb{V},-\gamma_{1}}^{(-)} \mathfrak{S}_{\mathbb{V},-\gamma_{2}}^{(-)}=\mathrm{id}
$$

Using the simplified notation $\mathfrak{S}_{\mathbb{V}, \gamma}^{(+)}=\mathfrak{S}_{\gamma}$ and the equality (8.5), the identity is written as

$$
\mathfrak{S}_{\gamma_{1}} \mathfrak{S}_{\gamma_{1}+\gamma_{2}} \mathfrak{S}_{\gamma_{2}}\left(\mathfrak{S}_{\gamma_{1}}\right)^{-1}\left(\mathfrak{S}_{\gamma_{2}}\right)^{-1}=\mathrm{id}
$$

or equivalently,

$$
\mathfrak{S}_{\gamma_{2}} \mathfrak{S}_{\gamma_{1}}=\mathfrak{S}_{\gamma_{1}} \mathfrak{S}_{\gamma_{1}+\gamma_{2}} \mathfrak{S}_{\gamma_{2}}
$$

This is the identity (1.2) by [DDP93].

\section{Appendix}

\section{A. Proof of Theorem 3.4}

Here we give a proof of Theorem 3.4. Let us recall the situation. We consider the case that the Stokes graph $G_{0}=G(\phi)$ has a unique saddle trajectory $\ell_{0}$, and it is a regular saddle trajectory. Note that, since there are no saddle trajectory other than $\ell_{0}$, other Stokes curves must flow into a point in $P_{\infty}$ at one end. To specify the situation, in addition to Figure 12, we take branch cuts and assign $\oplus$ and $\ominus$ as in Figure A1. (Note that we can show Theorem 3.4 in the same manner as presented here if the signs are assigned differently.) Then, the saddle class $\gamma_{0}$ associated with $\ell_{0}$ has the orientation shown in Figure A1,

Let $G_{ \pm \delta}=G\left(\phi_{ \pm \delta}\right)$ be the saddle reductions of $G_{0}$ (where $\delta>0$ is a sufficiently small number), and let $\mathcal{S}_{ \pm}\left[e^{V_{\gamma}}\right]$ (resp., $\mathcal{S}_{ \pm}\left[e^{W_{\beta}}\right]$ ) be the Borel sum of the Voros symbol $e^{V_{\gamma}}$ (resp., $e^{W_{\beta}}$ ) in the direction $\pm \delta$ (see Section 3.3). Here $\gamma \in H_{1}(\hat{\Sigma} \backslash \hat{P})$ is any cycle and $\beta \in H_{1}\left(\hat{\Sigma} \backslash \hat{P}_{0}, \hat{P}_{\infty}\right)$ is any path. Now we will show the equality

$$
\mathcal{S}_{-}\left[e^{W_{\beta}}\right]=\mathcal{S}_{+}\left[e^{W_{\beta}}\right]\left(1+\mathcal{S}_{+}\left[e^{V_{\gamma_{0}}}\right]\right)^{-\left\langle\gamma_{0}, \beta\right\rangle}, \quad \mathcal{S}_{-}\left[e^{V_{\gamma}}\right]=\mathcal{S}_{+}\left[e^{V_{\gamma}}\right]\left(1+\mathcal{S}_{+}\left[e^{V_{\gamma_{0}}}\right]\right)^{-\left(\gamma_{0}, \gamma\right)} .
$$

(i.e., the equality (3.10) ) on a domain containing $\{\eta \in \mathbb{R} \mid \eta \gg 1\}$.

Firstly, we show an important result for the Borel sums of the Voros symbols. 


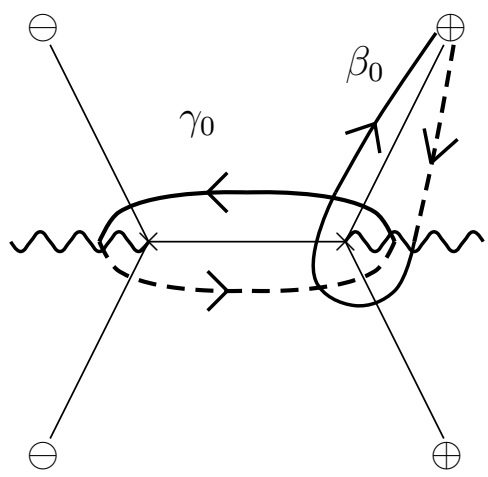

Figure A1. The saddle class $\gamma_{0} \in H_{1}(\hat{\Sigma} \backslash \hat{P})$ and a path $\beta_{0} \in H_{1}\left(\hat{\Sigma} \backslash \hat{P}_{0}, \hat{P}_{\infty}\right)$. The picture depicts a part of the Stokes graph $G_{0}$ near the saddle trajectory $\ell_{0}$.

Lemma A.1. If $\beta$ (resp., $\gamma$ ) does not intersect with the saddle trajectory $\ell_{0}$, the Borel sums $\mathcal{S}_{ \pm}\left[e^{W_{\beta}}\right]$ (resp., $\mathcal{S}_{ \pm}\left[e^{V_{\gamma}}\right]$ ) does not jump. That is, the equalities

$$
\mathcal{S}_{-}\left[e^{W_{\beta}}\right]=\mathcal{S}_{+}\left[e^{W_{\beta}}\right] \quad \text { if }\left\langle\gamma_{0}, \beta\right\rangle=0, \quad \mathcal{S}_{-}\left[e^{V_{\gamma}}\right]=\mathcal{S}_{+}\left[e^{V_{\gamma}}\right] \quad \text { if }\left(\gamma_{0}, \gamma\right)=0
$$

hold as analytic functions of $\eta$ on $\{\eta \in \mathbb{R} \mid \eta \gg 1\}$. Especially, the Borel sum of $e^{V_{\gamma_{0}}}$ does not jump.

Proof. It follows from the assumption and Corollary 2.21 that the Voros symbols $e^{W_{\beta}}$ and $e^{V_{\gamma}}$ are Borel summable (in the direction 0). Since the Stokes graph $G_{0}$ contains no other saddle trajectory than $\ell_{0}$, we can prove the statement in the same manner as in the proof of Lemma 3.3 .

Consequently, for a path $\beta$ which never intersects with $\ell_{0}$, both of the Borel sums $\mathcal{S}_{+}\left[e^{W_{\beta}}\right]$ and $\mathcal{S}_{-}\left[e^{W_{\beta}}\right]$ coincide with $\mathcal{S}\left[e^{W_{\beta}}\right]\left(=\mathcal{S}_{0}\left[e^{W_{\beta}}\right]\right)$. Similarly, we have $\mathcal{S}_{ \pm}\left[e^{V_{\gamma}}\right]=$ $\mathcal{S}\left[e^{V_{\gamma}}\right]$ for a cycle $\gamma$ if it never intersects with $\ell_{0}$. Below we write $\mathcal{S}_{ \pm}\left[e^{W_{\beta}}\right]=\mathcal{S}\left[e^{W_{\beta}}\right]$ etc. when a path or a cycle does not intersect with $\ell_{0}$.

The formula (A.2) is a part of the desired formula (A.1). In what follows we try to show (A.1) for the paths and the cycles which intersect with $\ell_{0}$. Note that, since any path $\beta \in H_{1}\left(\hat{\Sigma} \backslash \hat{P}_{0}, \hat{P}_{\infty}\right)$ and any cycle $\gamma \in H_{1}(\hat{\Sigma} \backslash \hat{P})$ can be written by a finite number of paths whose end-points are contained in $\hat{P}_{\infty}$ in the relative homology group $H_{1}\left(\hat{\Sigma} \backslash \hat{P}_{0}, \hat{P}_{\infty}\right)$ (see the proof of Lemma 3.3), it suffices to show (A.1) for any such a path $\beta \in H_{1}\left(\hat{\Sigma} \backslash \hat{P}_{0}, \hat{P}_{\infty}\right)$.

Lemma A.2. Let $\beta_{0} \in H_{1}\left(\hat{\Sigma} \backslash \hat{P}_{0}, \hat{P}_{\infty}\right)$ be the path depicted in Figure A1. Then, the following equality holds as analytic functions of $\eta$ on $\{\eta \in \mathbb{R} \mid \eta \gg 1\}$ :

$$
\mathcal{S}_{-}\left[e^{W_{\beta_{0}}}\right]=\mathcal{S}_{+}\left[e^{W_{\beta_{0}}}\right]\left(1+\mathcal{S}\left[e^{V_{\gamma_{0}}}\right]\right)
$$

Proof. Let us consider two connection problems for the WKB solutions indicated in Figure A2 which depicts a part of the Stokes graph $G_{+\delta}$ and $G_{-\delta}$. The first one is the connection problem from the Stokes region $D_{1}^{+}$to $D_{2}^{+}$in the Stokes graph $G_{+\delta}$, while 

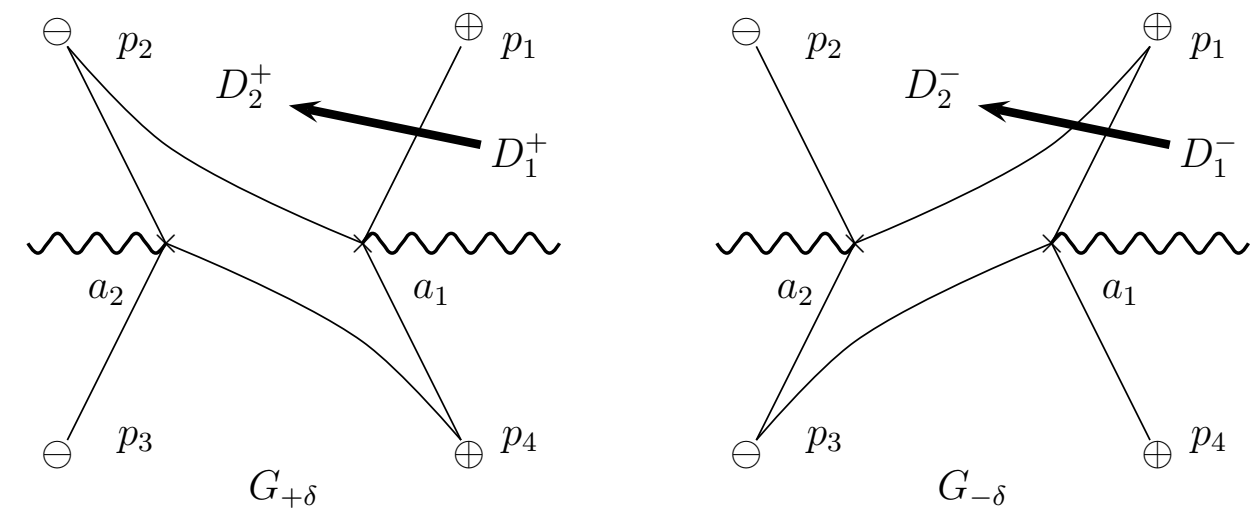

Figure A2. Two connection problems.

the second one is the connection problem from the Stokes region $D_{1}^{-}$to $D_{2}^{-}$in the Stokes graph $G_{-\delta}$ along the thick paths depicted in Figure A2.

Take the WKB solutions

$$
\psi_{ \pm, a_{1}}(z, \eta)=\frac{1}{\sqrt{S_{\text {odd }}(z, \eta)}} \exp \left( \pm \int_{a_{1}}^{z} S_{\text {odd }}(z, \eta) d z\right) .
$$

normalized at the turning point $a_{1}$ depicted in Figure A2. Since the saddle reductions $G_{ \pm \delta}$ are saddle-free, Corollary 2.21 ensures that the Borel sum of the WKB solutions are well-defined on each Stokes region of $G_{ \pm \delta}$. We denote by $\Psi_{ \pm, a_{1}}^{D_{1}^{+}}$etc. the Borel sum of $\psi_{ \pm, a_{1}}(z, \eta)$ in the Stokes region $D_{1}^{+}$etc. Then, using Theorem 2.25, we have the following formula for the first connection problem:

$$
\left\{\begin{array}{l}
\Psi_{+, a_{1}}^{D_{1}^{+}}=\Psi_{+, a_{1}}^{D_{2}^{+}}+i \Psi_{-, a_{1}}^{D_{2}^{+}} \\
\Psi_{-, a_{1}}^{D_{1}^{+}}=\Psi_{-, a_{1}}^{D_{2}^{+}}
\end{array}\right.
$$

On the other hand, in the second connection problem we have to cross two Stokes curves emanating from $a_{1}$ and $a_{2}$, respectively, as in Figure A2, In order to use Theorem 2.25 on the Stokes curve emanating from $a_{2}$, we need to change the normalization of the WKB solutions from (A.4) to

$$
\psi_{ \pm, a_{2}}(z, \eta)=\frac{1}{\sqrt{S_{\text {odd }}(z, \eta)}} \exp \left( \pm \int_{a_{2}}^{z} S_{\text {odd }}(z, \eta) d z\right)
$$

which is normalized at $a_{2}$. Using the relation

$$
\psi_{ \pm, a_{1}}(z, \eta)=\exp \left( \pm \int_{a_{1}}^{a_{2}} S_{\text {odd }}(z, \eta) d z\right) \psi_{ \pm, a_{2}}(z, \eta)=\exp \left( \pm \frac{1}{2} V_{\gamma_{0}}(\eta)\right) \psi_{ \pm, a_{2}}(z, \eta)
$$

(here $\gamma_{0}$ is the cycle depicted in Figure A1) of the WKB solutions with different normalizations, we have the following formula for the second connection problem (see [KT05, Section 3]):

$$
\left\{\begin{array}{l}
\Psi_{+, a_{1}}^{D_{1}^{-}}=\Psi_{+, a_{1}}^{D_{2}^{-}}+i\left(1+\mathcal{S}\left[e^{V_{\gamma_{0}}}\right]\right) \Psi_{-, a_{1}}^{D_{2}^{-}} \\
\Psi_{-, a_{1}}^{D_{1}^{-}}=\Psi_{-, a_{1}}^{D_{2}^{-}}
\end{array}\right.
$$


Thus, we obtain the two formulas (A.5) and (A.8).

Next, let us rewrite the formulas (A.5) and (A.8) to the formulas for the WKB solutions

$$
\psi_{ \pm, p_{1}}(z, \eta)=\frac{1}{\sqrt{S_{\text {odd }}(z, \eta)}} \exp \left\{ \pm\left(\eta \int_{a_{1}}^{z} \sqrt{Q_{0}(z)} d z+\int_{p_{1}}^{z} S_{\text {odd }}^{\text {reg }}(z, \eta) d z\right)\right\}
$$

normalized at $p_{1} \in \hat{P}_{\infty}$, which is an end-point of a Stokes curve emanating from $a_{1}$ as depicted in Figure A2, The WKB solutions (A.4) and (A.9) are related as

$$
\psi_{ \pm, a_{1}}(z, \eta)=\exp \left( \pm \int_{a_{1}}^{p_{1}} S_{\text {odd }}^{\mathrm{reg}}(z, \eta) d z\right) \psi_{ \pm, p_{1}}=\exp \left( \pm \frac{1}{2} W_{\beta_{0}}(\eta)\right) \psi_{ \pm, p_{1}}(z, \eta)
$$

where $\beta_{0}$ is the path designated in Figure A1, Therefore, it follows from (A.10), (A.5) and (A.8) that the following equalities hold:

$$
\begin{aligned}
& \left\{\begin{array}{l}
\Psi_{+, p_{1}}^{D_{1}^{+}}=\Psi_{+, p_{1}}^{D_{2}^{+}}+i \mathcal{S}_{+}\left[e^{-W_{\beta_{0}}}\right] \Psi_{-, p_{1}}^{D_{2}^{+}} \\
\Psi_{-, p_{1}}^{D_{1}^{+}}=\Psi_{-, p_{1}}^{D_{2}^{+}}
\end{array}\right. \\
& \left\{\begin{array}{l}
\Psi_{+, p_{1}}^{D_{1}^{-}}=\Psi_{+, p_{1}}^{D_{2}^{-}}+i\left(1+\mathcal{S}\left[e^{V_{\gamma_{0}}}\right]\right) \mathcal{S}_{-}\left[e^{-W_{\beta_{0}}}\right] \Psi_{-, p_{1}}^{D_{2}^{-}} \\
\Psi_{-, p_{1}}^{D_{-}^{-}}=\Psi_{-, p_{1}}^{D_{2}^{-}}
\end{array}\right.
\end{aligned}
$$

Taking $\delta>0$ sufficiently small, we may assume that, for a fixed $z_{1} \in D_{1}^{ \pm}$(resp., $z_{2} \in D_{2}^{ \pm}$) the path from $p_{1}$ to $z$, which normalize the WKB solution (A.9) when $z$ lies in a neighborhood of $z_{1}$ (resp., $z_{2}$ ), is admissible in any direction $\theta$ satisfying $-\delta \leq \theta \leq+\delta$. Therefore, Proposition 2.23 implies that

$$
\Psi_{ \pm, p_{1}}^{D_{1}^{+}}=\Psi_{ \pm, p_{1}}^{D_{1}^{-}}, \quad \Psi_{ \pm, p_{1}}^{D_{2}^{+}}=\Psi_{ \pm, p_{1}}^{D_{2}^{-}}
$$

holds as analytic functions of both $z$ and $\eta$ for a sufficiently large $\eta \gg 1$. Therefore, comparing the connection multipliers in (A.11) and (A.12) and using the equality (A.13), we obtain (A.3.

Remark A.3. Since the WKB solutions (A.4) are normalized along a path which intersects with the saddle trajectory $\ell_{0}$, we can not expect similar equalities as (A.13) holds for the Borel sums of (A.4).

The equality (A.3) is also one of the desired formula (A.1) since the intersection number is $\left\langle\gamma_{0}, \beta_{0}\right\rangle=-1$ as depicted in Figure A1. From this relation we can derive (A.1) for any path. The path $\beta_{0}$ in Figure A1 has the following decomposition in $H_{1}\left(\hat{\Sigma} \backslash \hat{P}_{0}, \hat{P}_{\infty}\right)$ :

$$
\beta_{0}=\beta_{p_{1}^{*}, p_{4}}+\beta_{p_{4}, p_{2}}+\beta_{p_{2}, p_{1}}
$$

as depicted in Figure A3. Here $p_{1}^{*}$ represents the point on the second sheet of $\hat{\Sigma}$ corresponding to $p_{1}$, and a dashed line is a path on the second sheet of $\hat{\Sigma}$. In the decomposition (A.14) the path $\beta_{p_{4}, p_{2}}$ intersects with the saddle trajectory $\ell_{0}$ and, on the 

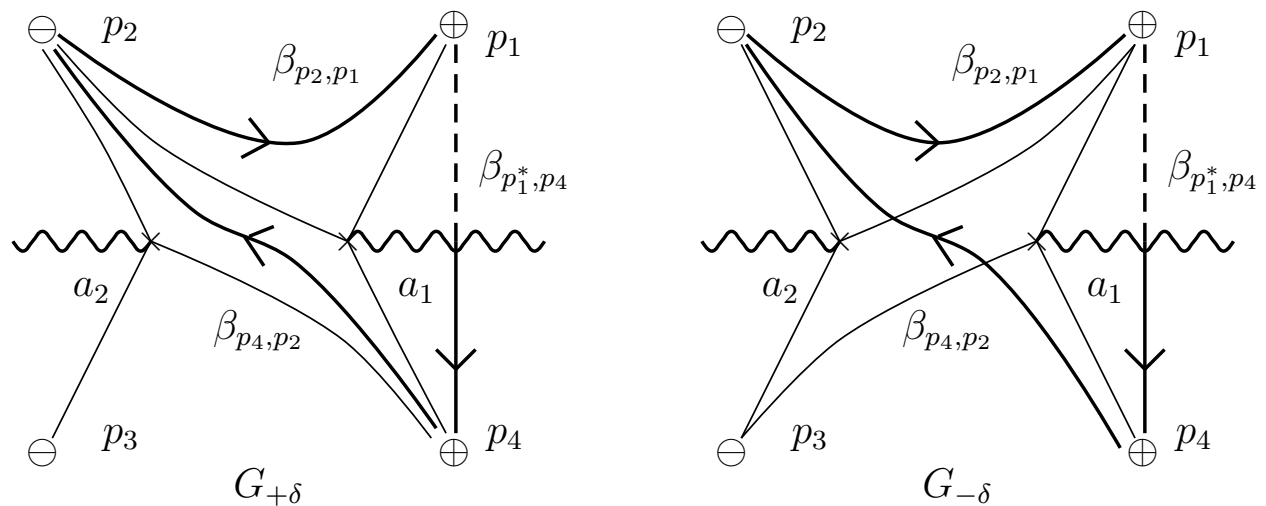

Figure A3. Decomposition of the path $\beta_{0}$.

other hand, the paths $\beta_{p_{1}^{*}, p_{4}}$ and $\beta_{p_{2}, p_{1}}$ never intersects with any saddle trajectories of $\phi$ since the Stokes graph $G_{0}$ does not has any saddle trajectory except for $\ell_{0}$. Thus, using the equality (A.2), the ratio of the Borel sums of the Voros symbol $e^{W_{\beta_{0}}}$ is given by

$$
\frac{\mathcal{S}_{-}\left[e^{W_{\beta_{0}}}\right]}{\mathcal{S}_{+}\left[e^{W_{\beta_{0}}}\right]}=\frac{\mathcal{S}\left[e^{W_{\beta_{1}^{*}, p_{4}}}\right] \mathcal{S}_{-}\left[e^{W_{\beta_{p_{4}}, p_{2}}}\right] \mathcal{S}\left[e^{W_{\beta_{p_{2}}, p_{1}}}\right]}{\mathcal{S}\left[e^{W_{\beta_{1}^{*}, p_{4}}}\right] \mathcal{S}_{+}\left[e^{W_{\beta_{p_{4}}, p_{2}}}\right] \mathcal{S}\left[e^{W_{\beta_{p_{2}}, p_{1}}}\right]}=\frac{\mathcal{S}_{-}\left[e^{W_{\beta_{p_{4}}, p_{2}}}\right]}{\mathcal{S}_{+}\left[e^{W_{\beta_{p_{4}}, p_{2}}}\right]} .
$$

Together with (A.3) we have the formula (A.1) for $\beta_{p_{4}, p_{2}}$ :

$$
\mathcal{S}_{-}\left[e^{W_{\beta_{p_{4}}, p_{2}}}\right]=\mathcal{S}_{+}\left[e^{W_{\beta_{p_{4}}, p_{2}}}\right]\left(1+\mathcal{S}\left[e^{V_{\gamma_{0}}}\right]\right) .
$$

Since any path and any cycle intersecting with $\ell_{0}$ can be expressed as a sum of $\pm \beta_{p_{4}, p_{2}}$ and some paths which never intersect with $\ell_{0}$. Thus, (A.1) holds for any path and any cycle. Thus we have proved Theorem 3.4 .

\section{B. Proof of Theorem 3.7}

Here we give a proof of Theorem 3.7. The proof presented here is different from that of [AIT], and a more sophisticated proof will be presented there.

\section{B.1. Settings}

Here we recall the situation and explain the idea for the proof of Theorem 3.7 .

We consider the case that the Stokes graph $G_{0}=G(\phi)$ has a unique saddle trajectory $\ell_{0}$, and it is a degenerate saddle trajectory around a double pole $p$. Denote by $D_{0}$ the degenerate ring domain whose boundary consists of $\ell_{0}$ and $p$. To specify the situation, in addition to Figure 12, we take branch cuts and assign $\oplus$ and $\ominus$ as in Figure B1. In this case we can not assign the sign for $p$ because there is no trajectory which flows to $p$ (recall that a degenerate ring domain is swept by closed trajectories). Then, the saddle class $\gamma_{0}$ associated with $\ell_{0}$ has the orientation shown in Figure B1. As indicated in Figure B1, $\gamma_{0}$ is $*$-equivalent to the sum of two closed cycles around 


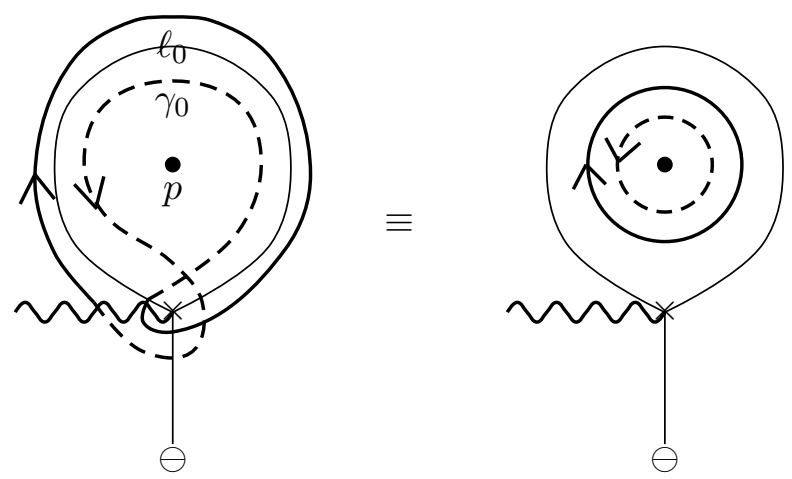

Figure B1. The saddle class $\gamma_{0} \in H_{1}(\hat{\Sigma} \backslash \hat{P})$. The picture depicts a part of the Stokes graph $G_{0}$ near the saddle trajectory $\ell_{0}$.

the double pole $p$; one lies on the first sheet and the other lies on the second sheet. Therefore, we have

$$
V_{\gamma_{0}}(\eta)=\oint_{\gamma_{0}} S_{\text {odd }}(z, \eta) d z=-4 \pi i \eta \operatorname{Res}_{z=p} \sqrt{Q_{0}(z)} d z
$$

(See (3.14).$)$ Moreover, $\gamma_{0}$ is contained in the kernel of the intersection form $(, \quad)$ on $H_{1}(\hat{\Sigma} \backslash \hat{P})$.

Fix a sufficiently small number $\delta_{0}>0$ and consider the saddle reductions $G_{ \pm \delta_{0}}=$ $G\left(\phi_{ \pm \delta_{0}}\right)$ of $G_{0}$. Let $\mathcal{S}_{ \pm}\left[e^{V_{\gamma}}\right]$ (resp., $\mathcal{S}_{ \pm}\left[e^{W_{\beta}}\right]$ ) be the Borel sum of the Voros symbol $e^{V_{\gamma}}$ (resp., $\left.e^{W_{\beta}}\right)$ in the direction $\pm \delta_{0}$. Here $\gamma \in H_{1}(\hat{\Sigma} \backslash \hat{P})$ is any cycle and $\beta \in H_{1}\left(\hat{\Sigma}_{\bar{P}} \hat{P}_{0}, \hat{P}_{\infty}\right)$ is any path. Now we will show the equalities

$$
\mathcal{S}_{-}\left[e^{W_{\beta}}\right]=\mathcal{S}_{+}\left[e^{W_{\beta}}\right]\left(1-\mathcal{S}_{+}\left[e^{V_{\gamma_{0}}}\right]\right)^{\left\langle\gamma_{0}, \beta\right\rangle}, \quad \mathcal{S}_{-}\left[e^{V_{\gamma}}\right]=\mathcal{S}_{+}\left[e^{V_{\gamma}}\right]
$$

(i.e., the equality (3.13) $)$ on a domain containing $\{\eta \in \mathbb{R} \mid \eta \gg 1\}$. Note that the Borel sum of $e^{V_{\gamma_{0}}}$ coincides with itself; $\mathcal{S}_{+}\left[e^{V_{\gamma_{0}}}\right]=e^{V_{\gamma_{0}}}$ since $V_{\gamma_{0}}$ is not a formal series but a scaler in view of (B.1). By the same argument in the proof of Lemma A.1, the equality (B.2) holds for any path $\beta$ and any cycle $\gamma$ which never intersect with $\gamma_{0}$. Especially, since $\left(\gamma_{0}, \gamma\right)=0$ for any cycle $\gamma$, (B.2) holds for any cycle $\gamma$. Therefore, it suffices to show (B.2) for any path $\beta \in H_{1}\left(\hat{\Sigma} \backslash \hat{P}_{0}, \hat{P}_{\infty}\right)$ intersecting with $\gamma_{0}$. The path $\beta_{p}$ depicted in Figure $\mathrm{B2}$ is a typical example of such a path.

Before the derivation of the formula $(\bar{B} .2)$, we give a remark on the property of Stokes curves in the saddle reductions of a degenerate saddle trajectory. Figure B3 depicts examples of Stokes curves for some directions. For any direction $\theta \neq 0$ near 0 , there exists a Stokes curve emanating from $a$ forms a logarithmic spiral and flows into $p$. As we vary the direction $\theta$, the Stokes curve hits any point $z \in D_{0}$. Moreover, as $\theta$ tends to 0 , any point $z \in D_{0}$ is hit by the Stokes curve infinitely many times, and the degenerate saddle trajectory appears as the limit $\theta \rightarrow 0$ of the Stokes curve.

Fix a point $z_{0} \in D_{0}$ ("reference point") and take a small disc $U$ containing $z_{0}$ and included in $D_{0}$. We assume that any point $U$ is not on the Stokes graphs $G_{ \pm \delta_{0}}$. 

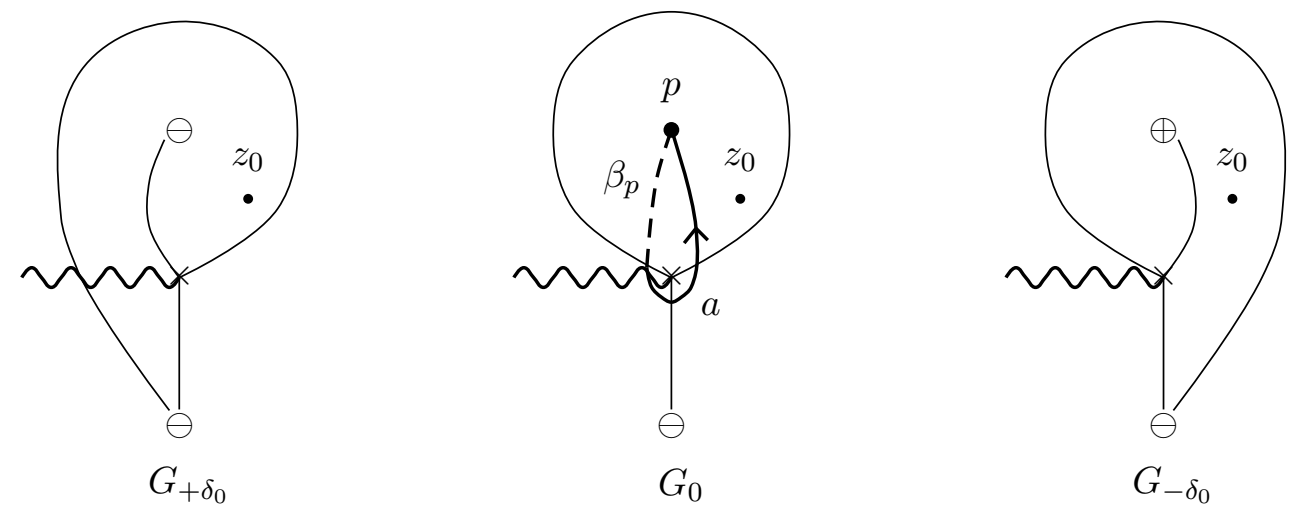

Figure B2. The saddle reduction of $G_{0}$. These pictures are schematic ones, and an actual saddle reduction is given in Figure B3.
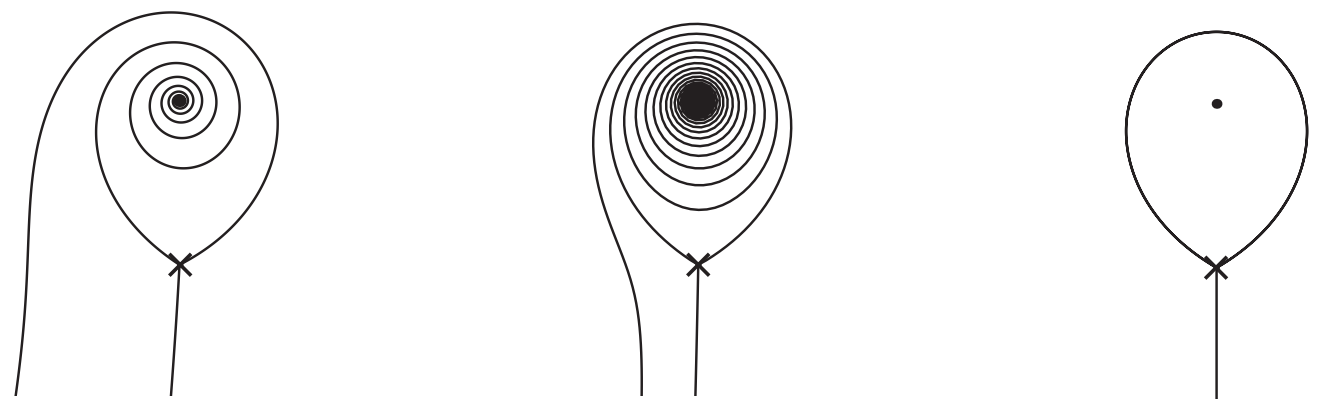

$\theta=+\delta_{0}$.

$$
0<\theta<+\delta_{0}
$$$$
\theta=0 .
$$

Figure B3. Stokes graphs in the limit $\theta \rightarrow+0$. Any point in the degenerate ring domain is hit by a Stokes curve infinitely many times as $\theta$ tends to 0. Similar pictures appear in the opposite limit $\theta \rightarrow-0$.

We will take certain WKB solutions defined on $U$, and compare their Borel sums in the directions $+\delta_{0}$ and $-\delta_{0}$. The comparison will be used to derive the formula (B.2) similarly to Appendix A,

However, the situation here is more complicated than that of Appendix A, Recall that, when $z$ lies on a Stokes curve in a direction $\theta$, WKB solutions may not be Borel summable in the direction $\theta$. In other wards, the Stokes phenomenon occurs to WKB solutions in such a direction $\theta$. Therefore, to compare the Borel sum in the directions $-\theta_{0}$ and $\theta_{0}$, we have to deal with infinitely many Stokes phenomena occurring to WKB solutions since the reference point $z_{0}$ is hit by Stokes curves infinitely many times. The situation is analyzed in Lemma B.2 below.

In our computation, we choose the reference point $z_{0}$ near the Stokes curve which forms a boundary of the degenerate horizontal strip around $p$ in the Stokes graphs $G_{+\delta_{0}}$ (see Figure B2). We may also assume the following: Take a straight half line starting from $p$ and passing through $z_{0}$. The point $z_{0}$ divides the half line into two parts; one is the segment between $p$ and $z_{0}$ and the other is a half line starting from $z_{0}$. 
Then, we assume that latter part never intersects with the Stokes curve which forms the logarithmic spiral in $G_{+\delta_{0}}$ and in $G_{-\delta_{0}}$. This guarantees that, the Borel sum of WKB solutions defined near $z_{0}$ can be analytically continued to the Stokes region which is adjacent to the degenerate horizontal strip along a straight path without crossing the logarithmic spirals (see Figure B5). We will also compare the result of the analytic continuations of Borel sum of WKB solutions along two paths indicated in Figure B5 in Lemma B.1 below. Combining Lemma B.1 and Lemma B.2, finally we obtain the formula (B.2).

\section{B.2. Derivation of the formulas in Theorem 3.7}

In the computation, we use the WKB solutions

$$
\psi_{ \pm, p}(z, \eta)=\frac{1}{\sqrt{S_{\text {odd }}(z, \eta)}} \exp \left\{ \pm\left(\eta \int_{a}^{z} \sqrt{Q_{0}(z)} d z+\int_{p}^{z} S_{\text {odd }}^{\text {reg }}(z, \eta) d z\right)\right\}
$$

defined on $U$, which are normalized at the double pole $p$ along a path from $p$ to $z$. Here $a$ is the turning point where $\ell_{0}$ emanates. For any $z \in U$, we can choose the path from $p$ to $z$ in (B.3) so that it never intersects with $\ell_{0}$, and hence $\psi_{ \pm, p}$ is Borel summable in the direction 0. Denote by $\Psi_{ \pm, p}^{\left(G_{0}\right)}$ the Borel sum defined on $U$. We will use $\Psi_{ \pm, p}^{\left(G_{0}\right)}(z, \eta)$ in order to compare the Borel sums $\Psi_{ \pm, p}^{\left(G_{+\delta_{0}}\right)}(z, \eta)$ and $\Psi_{ \pm, p}^{\left(G_{-\delta_{0}}\right)}(z, \eta)$ of $\psi_{ \pm, p}(z, \eta)$ in the direction $+\delta_{0}$ and $-\delta_{0}$ defined on $U$, respectively.

We also deal with another WKB solutions

$$
\psi_{ \pm}(z, \eta)=\frac{1}{\sqrt{S_{\text {odd }}(z, \eta)}} \exp \left( \pm \int_{a}^{z} S_{\text {odd }}(z, \eta) d z\right)
$$

which are normalized at the turning point $a$ since the Stokes phenomena for $\psi_{ \pm}$are easy to describe. We fix the normalization of (B.4) so that the path from $a$ to $z$ is defined by

$$
\int_{a}^{z} S_{\text {odd }}(z, \eta) d z=\int_{\gamma_{z}^{(1)}} S_{\text {odd }}(z, \eta) d z
$$

where the path $\gamma_{z}^{(1)}$ is depicted in Figure B4, Although we have illustrated the path $\gamma_{z}^{(1)}$ so that the one of its end point is the turning point $a$, the integral should be regarded as a contour integral (see Section 2.4). Then, the WKB solutions $\psi_{ \pm}$and $\psi_{ \pm, p}$ satisfy

$$
\psi_{ \pm}(z, \eta)=\exp \left( \pm \frac{1}{2} W_{\beta_{p}}(\eta)\right) \psi_{ \pm, p}(z, \eta)
$$

where $W_{\beta_{p}}$ is the Voros coefficient for the path $\beta_{p}$ depicted in Figure B2. Denote by $\Psi_{ \pm}^{\left(G_{+\delta_{0}}\right)}$ (resp., $\Psi_{ \pm}^{\left(G_{-\delta_{0}}\right)}$ ) the Borel sum of $\psi_{ \pm}$in the direction $+\delta_{0}$ (resp., $-\delta_{0}$ ) defined on $U$. Note that $\psi_{ \pm}$may not be Borel summable since the path $\gamma_{z}^{(1)}$ intersects with $\ell_{0}$.

Lemma B.1. The Borel sums $\Psi_{ \pm}^{\left(G_{+} \delta_{0}\right)}$ and $\Psi_{ \pm}^{\left(G_{-\delta_{0}}\right)}$ of $\psi_{ \pm}$are related as follows:

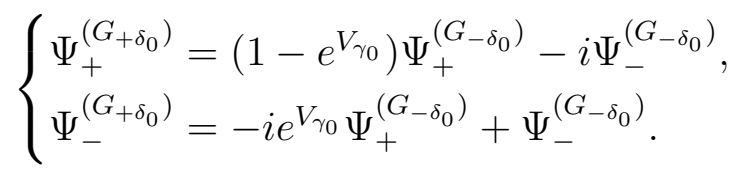




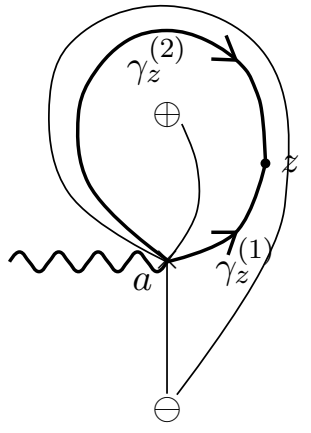

Figure B4. Normalization paths for WKB solutions (the picture depicts the Stokes graph in the direction $\left.-\delta_{0}\right)$.

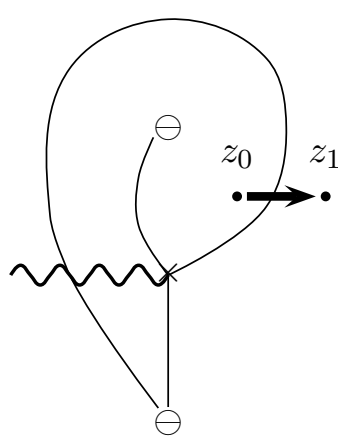

$G_{+\delta_{0}}$

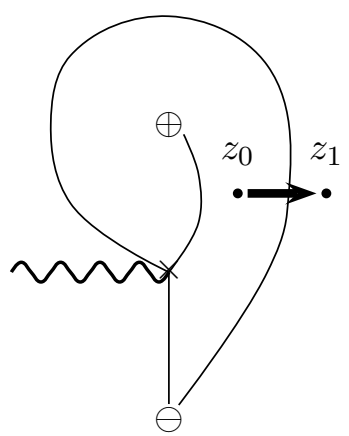

$G_{-\delta_{0}}$

Figure B5. Two connection problems.

Proof. Fix another reference point $z_{1}$ in the Stokes region adjacent to the Stokes region containing $z_{0}$ in $G_{ \pm \delta_{0}}$, and let us consider two connection problems for the WKB solutions from $z_{0}$ to $z_{1}$ as indicated in Figure B5. Taking $\delta_{0}$ sufficiently small, we may take $z_{1}$ so that it does not lie on any Stokes curves in any direction $\theta$ satisfying $-\delta_{0} \leq \theta \leq+\delta_{0}$. Let $\Psi_{ \pm, z_{1}}^{\left(G_{+\delta_{0}}\right)}$ (resp., $\Psi_{ \pm, z_{1}}^{\left(G_{-\delta_{0}}\right)}$ ) be the Borel sum of $\psi_{ \pm}$in the direction $+\delta_{0}$ (resp., $-\delta_{0}$ ) defined in a neighborhood of $z_{1}$. Then, Theorem 2.25 implies that, for the connection problem for $G_{+\delta_{0}}$, we have

$$
\left\{\begin{array}{l}
\Psi_{+}^{\left(G_{+\delta_{0}}\right)}=\Psi_{+, z_{1}}^{\left(G_{+\delta_{0}}\right)}-i \Psi_{-, z_{1}}^{\left(G_{+\delta_{0}}\right)} \\
\Psi_{-}^{\left(G_{+\delta_{0}}\right)}=\Psi_{-, z_{1}}^{\left(G_{+\delta_{0}}\right)}
\end{array}\right.
$$

Next let us consider the connection problem for $G_{-\delta_{0}}$. In this case, in order to use Theorem 2.25 on the Stokes curve in question, we have to change the normalization of the WKB solution; Theorem 2.25 is valid for the WKB solution normalized at $a$ along the Stokes curve in question. Namely, the formula (2.44) in Theorem 2.25 holds for the Borel sum of the WKB solutions

$$
\phi_{ \pm}(z, \eta)=\frac{1}{\sqrt{S_{\text {odd }}(z, \eta)}} \exp \left( \pm \int_{\gamma_{z}^{(2)}} S_{\text {odd }}(z, \eta) d z\right)
$$

whose integration path $\gamma_{z}^{(2)}$ is depicted in Figure B4, Thus we have

$$
\left\{\begin{array}{l}
\Phi_{+}^{\left(G_{-\delta_{0}}\right)}=\Phi_{+, z_{1}}^{\left(G_{-\delta_{0}}\right)} \\
\Phi_{-}^{\left(G_{-\delta_{0}}\right)}=\Phi_{-, z_{1}}^{\left(G_{-\delta_{0}}\right)}+i \Phi_{+, z_{1}}^{\left(G_{-\delta_{0}}\right)}
\end{array}\right.
$$

where $\Phi_{ \pm}^{\left(G_{-\delta_{0}}\right)}$ are the Borel sum of $\phi_{ \pm}$. Since $\psi_{ \pm}$and $\phi_{ \pm}$are related as

$$
\phi_{ \pm}=\exp \left( \pm \frac{1}{2} \int_{\gamma_{0}} S_{\text {odd }}(z, \eta) d z\right) \psi_{ \pm}=\exp \left( \pm \frac{1}{2} V_{\gamma_{0}}\right) \psi_{ \pm}
$$




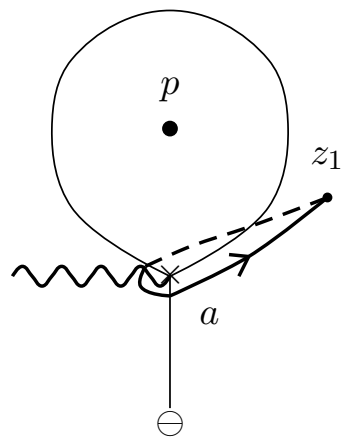

$G_{0}$

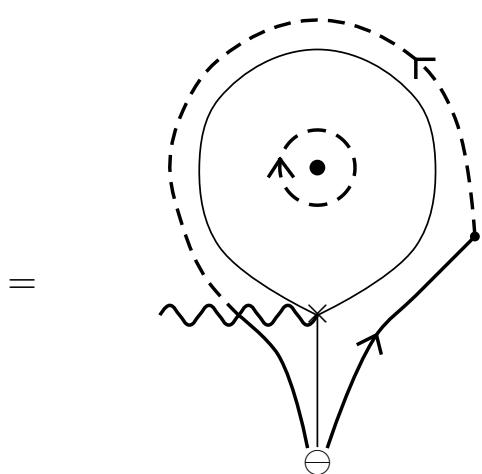

$G_{0}$

Figure B6. Deformation of a path.

we have the following formula for the Borel sum of $\psi_{ \pm}$:

$$
\left\{\begin{array}{l}
\Psi_{+}^{\left(G_{-\delta_{0}}\right)}=\Psi_{+, z_{1}}^{\left(G_{-\delta_{0}}\right)}, \\
\Psi_{-}^{\left(G_{-\delta_{0}}\right)}=\Psi_{-, z_{1}}^{\left(G_{-\delta_{0}}\right)}+i e^{V_{\gamma_{0}} \Psi_{+, z_{1}}^{\left(G_{-\delta_{0}}\right)} .}
\end{array}\right.
$$

Let us compare the right-hand sides of $(\overline{B .8})$ and $(\overline{B .12})$. Since we take $z_{1}$ outside of the saddle trajectory $\ell_{0}$, we can deform the path from $a$ to $z_{1}$ so that it never touches with $\ell_{0}$ (see Figure B6). Therefore, when $z$ lies on a neighborhood of $z_{1}$, the WKB solutions are Borel summable in the direction 0 even if the saddle trajectory $\ell_{0}$ appears. This implies that, as we vary the direction $\theta$ in $-\delta_{0} \leq \theta \leq+\delta_{0}$, no Stokes phenomenon occurs to $\psi_{ \pm}$near $z_{1}$. Thus we have

$$
\Psi_{ \pm, z_{1}}^{\left(G_{+\delta_{0}}\right)}=\Psi_{ \pm, z_{1}}^{\left(G_{-\delta_{0}}\right)}
$$

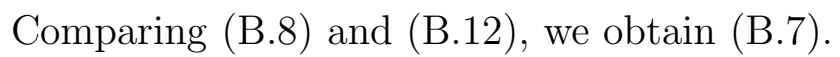

It follows from the first equality of (B.7) and (B.6), we have

$$
\mathcal{S}_{+}\left[e^{W_{\beta_{p}} / 2}\right] \Psi_{+, p}^{\left(G_{+\delta_{0}}\right)}=\left(1-e^{V_{\gamma_{0}}}\right) \mathcal{S}_{-}\left[e^{W_{\beta_{p}} / 2}\right] \Psi_{+, p}^{\left(G_{-\delta_{0}}\right)}-i \mathcal{S}_{-}\left[e^{-W_{\beta_{p}} / 2}\right] \Psi_{-, p}^{\left(G_{-\delta_{0}}\right)}
$$

The desired equality (B.2) will be follows from (B.14) and the following lemma.

Lemma B.2. (i) The Borel sum $\mathcal{S}_{\theta}\left[\psi_{+, p}\right]$ does not depend on the direction $\theta$ satisfying $0 \leq \theta \leq \delta_{0}$. Especially, we have

$$
\Psi_{+, p}^{\left(G_{+\delta_{0}}\right)}=\Psi_{+, p}^{\left(G_{0}\right)}
$$

for $z \in U$ and $\eta \in\{\eta \in \mathbb{R} \mid \eta \gg 1\}$. Similarly, we have

$$
\Psi_{-, p}^{\left(G_{-\delta_{0}}\right)}=\Psi_{-, p}^{\left(G_{0}\right)}
$$

for $z \in U$ and $\eta \in\{\eta \in \mathbb{R} \mid \eta \gg 1\}$. 
(ii) For the Borel sums $\Psi_{+, p}^{\left(G_{-\delta_{0}}\right)}$ and $\Psi_{+, p}^{\left(G_{0}\right)}$ of the WKB solution $\psi_{+, p}$, the equality

$$
\Psi_{+, p}^{\left(G_{-\delta_{0}}\right)}=\Psi_{+, p}^{\left(G_{0}\right)}+i\left(1-e^{V_{\gamma_{0}}}\right)^{-1} \mathcal{S}_{-}\left[e^{-W_{\beta_{p}}}\right] \Psi_{-, p}^{\left(G_{0}\right)}
$$

holds for $z \in U$ and $\eta \in\{\eta \in \mathbb{R} \mid \eta \gg 1\}$.

Proof. (i). As is explained in Subsection B.1, when we vary the direction $\theta$, any point $z \in D_{0}$ is hit by a Stokes curve, and which may yield the Stokes phenomenon to WKB solutions. However, such Stokes phenomena do not occur to the $\psi_{+, p}$ as long as we vary the direction $\theta$ in $0<\theta \leq \delta_{0}$ by the following reason. As we vary $\theta$ in $0<\theta \leq \delta_{0}$, the sign of $p$ never changes and is always $\ominus$. Hence, Stokes phenomena do not occur to $\psi_{+, p}$ even if $z_{0}$ lies on the Stokes curve. (See (2.46) in Remark 2.27) This guarantees that $\psi_{+, p}$ is Borel summable in any direction $\theta$ satisfying $0<\theta \leq \delta_{0}$. Furthermore, $\psi_{+, p}$ is also Borel summable in the direction 0 as we have noted above. Therefore, by the same argument in the proof of Proposition 2.23, we have proved the equality (B.15).

On the other hand, since the sign of $p$ in the Stokes graph of direction $\theta$ satisfying $-\delta_{0} \leq \theta<0$ is always $\oplus$, the Stokes phenomenon do not occur to $\psi_{-, p}$. (See (2.45) in Remark 2.27, ) Thus the equality (B.16) is derived in the same manner.

(ii). In the proof, we use a different expression of the Borel sum of WKB solutions. Let us write the WKB solution $\psi_{ \pm, p}$ in the following form:

$$
\psi_{ \pm, p}(z, \eta)=\exp ( \pm \eta s(z)) \sum_{k=0}^{\infty} \eta^{-k-1 / 2} \psi_{ \pm, k}(z), \quad s(z)=\int_{a}^{z} \sqrt{Q_{0}(z)} d z
$$

Then, the Borel sum of $\psi_{ \pm, p}$ in a direction $\theta$ is given by

$$
\int_{\mp s(z)}^{\infty e^{-i \theta}} e^{-\eta y} \psi_{ \pm, B}(z, y) d y
$$

where

$$
\psi_{ \pm, B}(z, y)=\sum_{k=0}^{\infty} \frac{\psi_{ \pm, k}(z)}{\Gamma(k+1 / 2)}(y \pm s(z))^{k-1 / 2}
$$

is the Borel transform of $\psi_{ \pm, p}$. The expression (B.19) coincides with the definition of the Borel sum (of formal series with exponential factors) adopted in Definition 2.10, The difference of the Borel sums $\Psi_{+, p}^{\left(G_{-\delta_{0}}\right)}$ and $\Psi_{+, p}^{\left(G_{0}\right)}$ in question is that of the path of Laplace integral in (B.19). That is, $\Psi_{+, p}^{\left(G_{-\delta_{0}}\right)}$ is given by the Laplace transform of $\psi_{+, B}(z, y)$ along the half line $L_{+\delta_{0}}=\left\{-s(z)+r e^{i \delta_{0}} \in \mathbb{C} \mid r \geq 0\right\}$, while $\Psi_{+, p}^{\left(G_{0}\right)}$ is given by the Laplace transform of the same function $\psi_{+, B}(z, y)$ along the half line $L_{0}=\{-s(z)+r \in \mathbb{C} \mid r \geq 0\}$ in the $y$-plane.

To compare the Borel sums $\Psi_{+, p}^{\left(G_{-\delta_{0}}\right)}$ and $\Psi_{+, p}^{\left(G_{0}\right)}$, we investigate the Stokes phenomena occurring in directions in $0<\theta \leq \delta_{0}$. Contrary to the case of (i) of Lemma B.2, when a Stokes curve in a direction $\theta$ satisfying $0<\theta \leq \delta_{0}$ hits a point $z$, the Stokes phenomenon occurs to $\psi_{+, p}$ since the sign at $p$ is $\oplus$ when $-\delta_{0} \leq \theta<0$ (see (2.43) ). In other wards, 


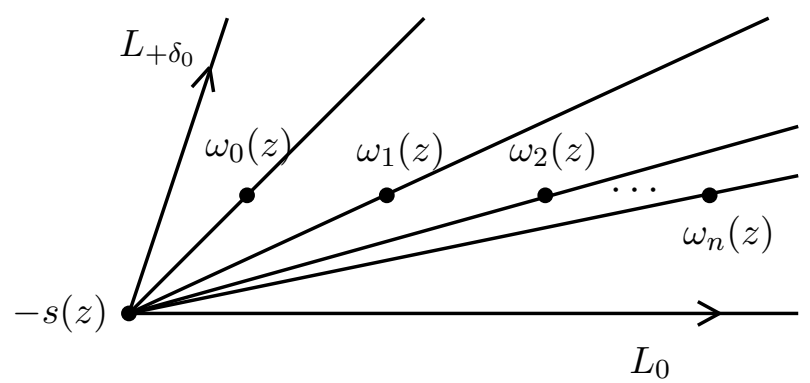

Figure B7. Singular points of $\psi_{+, B}(z, y)$ in the $y$-plane.
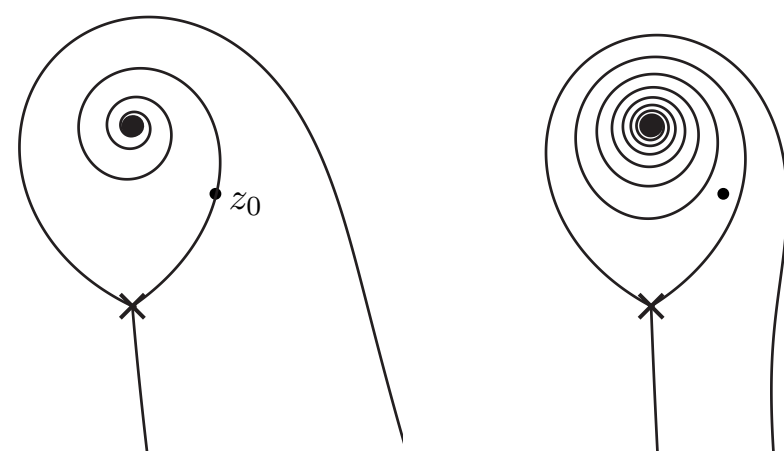

$\theta=\theta_{n-1}$.

$\theta_{n-1}<\theta<\theta_{n}$.

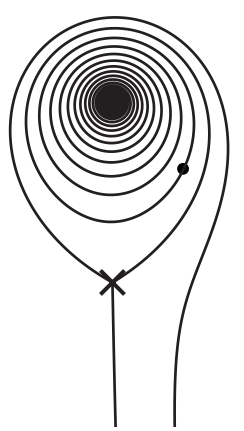

$\theta=\theta_{n}$.

Figure B8. The directions $\theta_{n}$. Here $\theta$ is the directions of Stokes curves. These pictures depict the case of $n=1$.

when a point $z$ lies on a Stokes curve in a direction $\theta$, a singular point of $\psi_{+, B}(z, y)$ lies on the half line $\left\{-s(z)+r e^{-i \theta} \in \mathbb{C} \mid r \geq 0\right\}$. (See (2.45) in Remark 2.27). As is explained in Subsection B.1, a Stokes curve hits a point $z \in U$ infinitely many times when $\theta$ tends to 0 . Therefore, $\psi_{+, B}(z, y)$ has infinitely many singular points $\left\{\omega_{n}(z)\right\}_{n=0}^{\infty}$ in the $y$-plane (see Figure B7), and these singular points cause the Stokes phenomena for $\psi_{+, p}$. Hence, we have to deal with the infinitely many Stokes phenomena to compare the Borel sums $\Psi_{+, p}^{\left(G_{-\delta_{0}}\right)}$ and $\Psi_{+, p}^{\left(G_{0}\right)}$.

Let $\left\{\theta_{n}\right\}_{n=0}^{\infty}$ be the increasing sequence of directions in $-\delta_{0}<\theta<0$ such that the reference point $z_{0}$ lies on a Stokes curve in the directions. These directions depend on $z_{0}$, and are characterized by the following conditions:

- For any $n \geq 0$, the point $z_{0}$ lies on the Stokes curve in the direction $\theta_{n}$ emanating from $a$ and ending at $p$. The Stokes curve reaches at $z_{0}$ after turning around $p$ $n$-times (see Figure B8).

- For any $n \geq 0$ and any $\theta$ satisfying $\theta_{n-1}<\theta<\theta_{n}$, $z_{0}$ does not lie on the Stokes curve in the direction $\theta$. Here we take $\theta_{-1}=-\delta_{0}$.

The direction $\theta_{n}$ is nothing but $-\arg \left(s\left(z_{0}\right)+\omega_{n}\left(z_{0}\right)\right)$ (see Figure B7).

For any $n \geq 0$, define the Borel sum $\Psi_{ \pm}[n]$ of the WKB solutions $\psi_{ \pm}$defined on $U$ as 
follows. Fix a direction $\theta$ satisfying $\theta_{n-1}<\theta<\theta_{n}$. Since $z_{0}$ does not lie on Stokes curves in the direction $\theta$, there exists a small disc $U^{\prime} \subset U$ containing $z_{0}$ and any point in $U^{\prime}$ does not lie on Stokes curves in the direction $\theta$. Then, take the Borel sum $\Psi_{ \pm}[n]$ in the direction $\theta$ defined on $U^{\prime}$. Denote by the same letter $\Psi_{ \pm}[n]$ the analytic continuation of the Borel sum to $U$. Then, $\Psi_{ \pm}[n]$ are independent of the choice of direction $\theta$ satisfying $\theta_{n-1}<\theta<\theta_{n}$ since they give the same germ of holomorphic function at $z_{0}$. Define the Borel sum $\Psi_{ \pm, p}[n]$ of $\psi_{ \pm, p}$ defined on $U$ by the same manner. Especially, $\Psi_{ \pm}[0]=\Psi_{ \pm}^{\left(G_{-\delta_{0}}\right)}$ and $\Psi_{ \pm, p}[0]=\Psi_{ \pm, p}^{\left(G_{-\delta_{0}}\right)}$ hold.

By a similar computation as (B.12), the factor $e^{n V_{\gamma_{0}}}$ appears in the coefficient of the formula between $\Psi_{ \pm}[n]$ and $\Psi_{ \pm}[n+1]$ for each $n \geq 0$ as follows:

$$
\left\{\begin{array}{l}
\Psi_{+}[n]=\Psi_{+}[n+1]+i e^{n V_{\gamma_{0}}} \Psi_{-}[n+1], \\
\Psi_{-}[n]=\Psi_{-}[n+1]\left(=\Psi_{-}[0]\right)
\end{array}\right.
$$

This formula is translated to the formula for $\Psi_{ \pm, p}[n]$ as follows:

$$
\left\{\begin{array}{l}
\Psi_{+, p}[n]=\Psi_{+, p}[n+1]+i e^{n V_{\gamma_{0}} \mathcal{S}_{-}\left[e^{-W_{\beta_{p}}}\right] \Psi_{-, p}[n+1],} \\
\Psi_{-, p}[n]=\Psi_{-, p}[n+1]\left(=\Psi_{-, p}[0]\right) .
\end{array}\right.
$$

Here the factor $\mathcal{S}_{-}\left[e^{-W_{\beta_{p}}}\right]$ appears as the consequence of the difference (B.6) of the normalization between $\psi_{ \pm}$and $\psi_{ \pm, p}$. Since the difference of $\Psi_{+, p}[n]$ and $\Psi_{+, p}[n+1]$ are expressed as the integral along the contour $C_{n}$ encircling the singular point $\omega_{n}(z)$ (see Figure (B9), the equality (B.22) implies that

$$
\int_{C_{n}} e^{-\eta y} \psi_{+, B}(z, y) d y=i e^{n V_{\gamma_{0}}} \mathcal{S}_{-}\left[e^{-W_{\beta_{p}}}\right] \Psi_{-, p}^{\left(G_{0}\right)} .
$$

Here we have used (B.16). Therefore, we have

$$
\begin{aligned}
\Psi_{+, p}^{\left(G_{-\delta_{0}}\right)}-\Psi_{+, p}^{\left(G_{0}\right)} & =\sum_{n=0}^{\infty} \int_{C_{n}} e^{-\eta y} \psi_{+, B}(z, y) d y \\
& =i\left(\sum_{n=0}^{\infty} e^{n V_{\gamma_{0}}}\right) \mathcal{S}_{-}\left[e^{-W_{\beta_{p}}}\right] \Psi_{-, p}^{\left(G_{0}\right)} .
\end{aligned}
$$

Thus, as the consequence of the infinitely many Stokes phenomena, the infinite sum $\sum_{n=0}^{\infty} e^{n V_{\gamma_{0}}}$ appears in the difference between $\Psi_{+, p}^{\left(G_{-\delta_{0}}\right)}$ and $\Psi_{+, p}^{\left(G_{0}\right)}$. The infinite series converges and the factor $\left(1-e^{V_{\gamma_{0}}}\right)^{-1}$ appears since the real part of $\oint_{\gamma_{0}} \sqrt{Q_{0}(z)} d z$ is negative. Thus we obtain (B.17).

Now we derive the desired formula $(\underline{B .2})$ for the path $\beta_{p}$. Substituting (B.15), (B.16) and (B.17) into (B.14), we obtain

$$
\mathcal{S}_{+}\left[e^{W_{\beta_{p}} / 2}\right] \Psi_{+, p}^{\left(G_{0}\right)}=\left(1-e^{V_{\gamma_{0}}}\right) \mathcal{S}_{-}\left[e^{W_{\beta_{p}} / 2}\right] \Psi_{+, p}^{\left(G_{0}\right)} .
$$

Note that the coefficient of $\Psi_{-, p}^{\left(G_{0}\right)}$ has been canceled out. Hence we have

$$
\mathcal{S}_{-}\left[e^{W_{\beta_{p}}}\right]=\mathcal{S}_{+}\left[e^{W_{\beta_{p}}}\right]\left(1-e^{V_{\gamma_{0}}}\right)^{-2}
$$




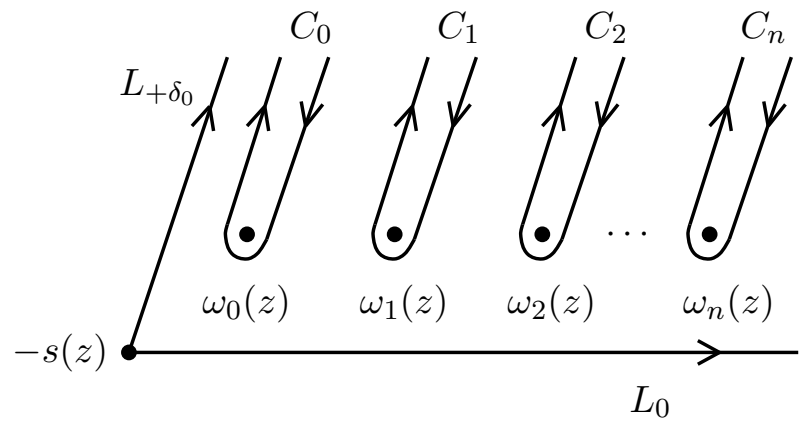

Figure B9. Contour integrals representing the difference of $\Psi_{+, p}^{\left(G_{-\delta_{0}}\right)}$ and $\Psi_{+, p}^{\left(G_{0}\right)}$.

This is the desired formula (B.2) for the path $\beta_{p}$ since $\left\langle\gamma_{0}, \beta_{p}\right\rangle=-2$.

The formula (B.2) for general path $\beta$ is derived from (B.26). For example, let us take a path $\beta_{a}$ from $p$ to another pole in Figure B10, which satisfies $\left\langle\gamma_{0}, \beta_{a}\right\rangle=-1$. Note that, as indicated in Figure B10, the path $\beta_{p}$ have the decomposition $\beta_{p} \equiv 2 \beta_{a}+\beta_{b}$ under the $*$-equivalence. Here $\beta_{b}$ is a path which never intersects with $\ell_{0}$, and hence

$$
\mathcal{S}_{-}\left[e^{W_{\beta_{b}}}\right]=\mathcal{S}_{+}\left[e^{W_{\beta_{b}}}\right]
$$

Then, it follows from (B.26) that

$$
\mathcal{S}_{-}\left[e^{2 W_{\beta_{a}}}\right]=\mathcal{S}_{+}\left[e^{2 W_{\beta_{a}}}\right]\left(1-e^{V_{\gamma_{0}}}\right)^{-2}
$$

holds. Taking the square root, we have

$$
\mathcal{S}_{-}\left[e^{W_{\beta_{a}}}\right]= \pm \mathcal{S}_{+}\left[e^{W_{\beta_{a}}}\right]\left(1-e^{V_{\gamma_{0}}}\right)^{-1} .
$$

We can conclude the sign \pm in $(\overline{B .29})$ is + because both of $\mathcal{S}_{-}\left[e^{W_{\beta_{a}}}\right]$ and $\mathcal{S}_{+}\left[e^{W_{\beta_{a}}}\right]$ have the same asymptotic expansion $e^{W_{\beta_{a}}}$ when $\eta \rightarrow+\infty$. Thus we have (B.2) for the path $\beta_{a}$. Since any path intersecting with $\ell_{0}$ can be expressed as a sum of $\pm \beta_{a}$ and some paths which never intersect with $\ell_{0}$. Thus, (B.2) holds for any path, and we have proved Theorem 3.7 .

\section{References}

[AIT] T. Aoki, K. Iwaki, and T. Takahashi, Exact WKB analysis of Schrödinger equation with a Stokes curve of loop type, in preparation.

[AKT91] T. Aoki, T. Kawai, and Y. Takei, The Bender-Wu analysis and the Voros theory, ICM-90 Satellite Conf. Proc. "Special Functions", Springer-Verlag, 1991, pp. 1-29.

[AT13] T. Aoki and M. Tanda, Borel sum of Voros coefficients of hypergeometric differential equation with a large parameter, RIMS Kôkyûroku 1861 (2013), 17-24.

[BFZ05] A. Berenstein, S. Fomin, and A. Zelevinsky, Cluster algebras III: upper bounds and double Bruhat cells, Duke Math. J. 126 (2005), 1-52; arXiv:math/035434 [math.RT].

[BS13] T. Bridgeland and I. Smith, Quadratic differentials as stability conditions, 2013, arXiv:1302.7030 [math.AG].

[Cir13] M. Cirafici, Line defects and (framed) BPS quivers, JHEP 11 (2013), 141; arXiv:1307.713 [hep-th]. 

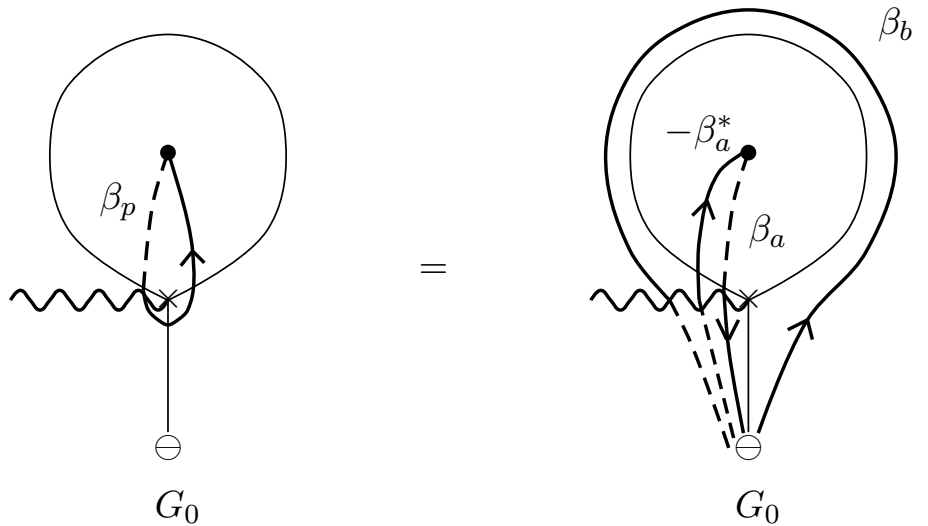

Figure B10. The path $\beta_{p}$ and a typical path $\beta_{a}$ which intersects $\ell_{0}$.

[Cos08] O. Costin, Asymptotics and Borel Summability, Monographs and surveys in pure and applied mathematics, vol. 141, Chapmann and Hall/CRC, 2008.

[DDP93] E. Delabaere, H. Dillinger, and F. Pham, Résurgence de Voros et périodes des courbes hyperelliptiques, Ann. Inst. Fourier (Grenoble) 43 (1993), 163-199.

[DP99] E. Delabaere and F. Pham, Resurgent methods in semi-classical asymptotics, Ann. Inst. Henri Poincaré 71 (1999), 1-94.

[DWZ10] H. Derksen, J. Weyman, and A. Zelevinsky, Quivers with potentials and their representations II: Applications to cluster algebras, J. Amer. Math. Soc. 23 (2010), 749-790; arXiv:0904.0676 [math.RA].

[Eca84] J. Ecallé, Cinq applications des fonctions résurgentes, 1984, Preprint, Prepub. Math. d'Orsay, 84T62, $110 \mathrm{pp}$.

[Fed93] M. V. Fedoryuk, Asymptotic analysis: linear ordinary differential equations, Springer-Verlag, 1993.

[FG06] V. V. Fock and A. B. Goncharov, Moduli spaces of local systems and higher Teichmüller theory, Publ. Math. IHES 103 (2006), 1-211, arXiv:math/0311149 [math.AG].

[FG07] _ Dual Teichmüller and lamination spaces, Handbook of Teichüller theory, Vol. I, Eur. Math. Soc., 2007, pp. 647-684, arXiv:math/0510312 [math.DG].

[FG09a] Cluster ensembles, quantization and the dilogarithm, Annales Sci. de l'École Norm. Sup. 42 (2009), 865-930; arXiv:math/0311245 [math.AG].

[FG09b] _ Cluster ensembles, quantization and the dilogarithm II: The intertwiner, Prog. Math. 269 (2009), 655-673; arXiv:math.0702398 [math.AG].

[FK94] L. D. Faddeev and R. M. Kashaev, Quantum dilogarithm, Mod. Phys. Lett. A9 (94), 427434; arXiv:hep-th/9310070.

[FST08] S. Fomin, M. Shapiro, and D. Thurston, Cluster algebras and triangulated surfaces. Part I: Cluster complexes, Acta Math. 201 (2008), 83-146; arXiv:math/0608367 [math.RA].

[FT12] S. Fomin and D. Thurston, Cluster algebras and triangulated surfaces. Part II: Lambda lengths, 2012, arXiv:1210.5569.

[FZ02] S. Fomin and A. Zelevinsky, Cluster algebras I. Foundations, J. Amer. Math. Soc. 15 (2002), 497-529 (electronic); arXiv:math/0104151 [math.RT].

[FZ03] _ Cluster algebras II. Finite type classification, Invent. Math. 154 (2003), 63-121; arXiv:math/0208229 [math.RA].

[FZ07] _ Cluster algebras IV. Coefficients, Compositio Mathematica 143 (2007), 112-164; arXiv:math/0602259 [math.RT].

[Get09] A. Getmanenko, Shatalov-Sternin's construction of complex WKB solutions and the associated Riemann surface, 2009, arXiv:0907.2934 [math.CA]. 
[Get11] Shatalov-Sternin's construction of complex WKB solutions and the choice of integration paths, 2011, arXiv:1111.6325 [math.CA].

[GMN13] D. Gaiotto, G. W. Moore, and A. Neitzke, Wall-crossing, Hitchin systems, and the WKB approximation, Adv. in Math. 234 (2013), 239-403; arXiv:0907.3987 [hep-th].

[GSV05] M. Gekhtman, M. Shapiro, and A. Vainshtein, Cluster algebras and Weil-Petersson forms, Duke Math. J. 127 (2005), 291-311; arXiv:math/0309138 [math.QA].

[GT11] A. Getmanenko and D. Tamarkin, Microlocal properties of sheaves and complex WKB, 2011, arXiv:1111.6325 [math-ph].

[Hat91] A. Hatcher, On triangulations of surfaces, Topololy Appl. 40 (1991), 189-194.

[IIK $\left.{ }^{+} 13 \mathrm{a}\right]$ R. Inoue, O. Iyama, B. Keller, A. Kuniba, and T. Nakanishi, Periodicities of $T$ and $Y$ systems, dilogarithm identities, and cluster algebras I: Type $B_{r}$, Publ. RIMS 49 (2013), 1-42; arXiv:1001.1880 [math.QA].

$\left[\mathrm{IIK}^{+} 13 \mathrm{~b}\right] \_$, Periodicities of $T$ and $Y$-systems, dilogarithm identities, and cluster algebras II: Types $C_{r}, F_{4}$, and $G_{2}$, Publ. RIMS 49 (2013), 43-85; arXiv:1001.1881 [math.QA].

[Kel10] B. Keller, Cluster algebras, quiver representations and triangulated categories, Triangulated categories (T. Holm, P. Jørgensen, and R. Rouquier, eds.), Lecture Note Series, vol. 375, London Mathematical Society, Cambridge University Press, 2010, pp. 76-160; arXiv:0807.1960 [math.RT].

[Kel11] B. Keller, On cluster theory and quantum dilogarithm identities, Representations of algebras and related topics (A. Skowroński and K. Yamagata, eds.), EMS Series of Congress Reports, European Mathematical Society, 2011, pp. 85-116; arXiv:1102.4148 [math.RT].

[KN11] R. M. Kashaev and T. Nakanishi, Classical and quantum dilogarithm identities, SIGMA 7 (2011), 102, 29 pages, arXiv:1104.4630 [math.QA].

[Koi00] T. Koike, On the exact WKB anlysis of second order linear ordinary differential equations with simple poles, Publ. RIMS (2000), 297-319.

[KS] T. Koike and R. Schäfke, On the Borel summability of WKB solutions of Schrödinger equations with polynomial potentials and its application, in preparation; also Talk given by T. Koike in the RIMS workshop "Exact WKB analysis - Borel summability of WKB solutions", September, 2010.

[KS08] M. Kontsevich and Y. Soibelman, Stability structures, Donaldson-Thomas invariants and cluster transformations, 2008, arXiv:0811.2435 [math.AG].

[KS10] _ Motivic Donaldson-Thomas invariants: summary of results, Contemp. Math. 527 (2010), 55-89; arXiv:0910.4315 [math.AG].

[KT05] T. Kawai and Y. Takei, Algebraic analysis of sigular perturbation theory, Translations of mathematical monographs, no. 227, American Mathematical Society, 2005.

[LF12] D. Labardini-Fragoso, Quivers with potentials associated to triangulated surfaces, Part IV: Removing boundary assumptions, 2012, arXiv:1206.1798 [math.CO].

[Nag11] K. Nagao, Quantumd dilogarithm identities, RIMS Kôkyûroku Bessatsu B28 (2011), 165170.

[Nag13] K. Nagao, Donaldson-Thomas theory and cluster algebras, Duke Math. J. 7 (2013), 13131367; arXiv:1002.4884 [math.AG].

[Nak12] T. Nakanishi, Tropicalization method in cluster algebras, Contemp. Math. 580 (2012), 95115; arXiv:1110.5472 [math.QA].

[NS12] T. Nakanishi and S. Stella, Wonder of sine-Gordon $Y$-systems, 2012, preprint version in arXiv:1212.6853.

[Pla11] P. Plamondon, Cluster algebras via cluster categories with infinite-dimensional morphism spaces, Compos. Math. 147 (2011), 1921-1954; arXiv:1004.0830 [math.RT].

[Qiu14] Y. Qiu, On the spheciral twists on 3-Calabi-Yau categories from marked surfaces, 2014, arXiv:1407.0806.

[SS09] D. Speyer and B. Sturmfels, Tropical mathematics, Mathematics Magazine 82 (2009), 163173; arXiv:math/0408099 [math.CO]. 
[Str84] K. Strebel, Quadratic differentials, Springer-Verlag, 1984.

[Tak08] Y. Takei, Sato's conjecture for the Weber equation and transformation theory for Schrödinger equations with a merging pair of turning points, RIMS Kôkyûroku Bessatsu B10 (2008), 205-224.

[Vor83] A. Voros, The return of the quartic oscillator. the complex WKB method, Ann. Inst. Henri Poincaré 39 (1983), 211-338.

[Xie12] D. Xie, BPS spectrum, wall crossing and quantum dilogarithm identity, 2012, arXiv:1211.707 [hep-th]. 